\title{
Evaluating the Potential of Methyl Jasmonate Application as an Agricultural Practice on Phytochemicals in Brassica Vegetables: Sensory Quality, Cooking, and Cost-Benefit Analysis
}

Yu-Chun Chiu

yuchiu@mix.wvu.edu

Follow this and additional works at: https://researchrepository.wvu.edu/etd

Part of the Food Chemistry Commons, and the Horticulture Commons

\section{Recommended Citation}

Chiu, Yu-Chun, "Evaluating the Potential of Methyl Jasmonate Application as an Agricultural Practice on Phytochemicals in Brassica Vegetables: Sensory Quality, Cooking, and Cost-Benefit Analysis" (2019). Graduate Theses, Dissertations, and Problem Reports. 7471.

https://researchrepository.wvu.edu/etd/7471

This Dissertation is protected by copyright and/or related rights. It has been brought to you by the The Research Repository @ WVU with permission from the rights-holder(s). You are free to use this Dissertation in any way that is permitted by the copyright and related rights legislation that applies to your use. For other uses you must obtain permission from the rights-holder(s) directly, unless additional rights are indicated by a Creative Commons license in the record and/ or on the work itself. This Dissertation has been accepted for inclusion in WVU Graduate Theses, Dissertations, and Problem Reports collection by an authorized administrator of The Research Repository @ WVU.

For more information, please contact researchrepository@mail.wvu.edu. 
Evaluating the Potential of Methyl Jasmonate Application as an Agricultural Practice on Phytochemicals in Brassica Vegetables: Sensory Quality, Cooking, and Cost-Benefit Analysis

\author{
Yu-Chun Chiu
}

\author{
A doctoral dissertation submitted to the \\ Davis College of Agriculture, Natural Resources and Design \\ at West Virginia University \\ In Partial Fulfillment of the Requirements for the degree of \\ Doctor of Philosophy \\ in \\ Plant and Soil Sciences \\ Kang-Mo Ku, Ph.D., Chair \\ Nicole Waterland, Ph.D., Co-Chair \\ Kristen Matak, Ph.D. \\ Xiaoli L. Etienne, Ph.D. \\ Jonathan Cumming, Ph.D.
}

Division of Plant and Soil Sciences

Morgantown, West Virginia

2019

Keywords: Methyl jasmonate, Brassica, glucosinolate, bitterness 


\section{ABSTRACT}

\section{Evaluating the Potential of Methyl Jasmonate Application as an Agricultural Practice on Brassica Vegetables: Sensory Quality, Cooking, and Cost-benefit Analysis}

\section{Yu-Chun Chiu}

In the United States, Brassica vegetables, including broccoli (Brassica oleracea var. italica) and kale (Brassica oleracea var. acephala or Brassicae napus var. pabularia), are widely consumed and are easily accessible in farmer's market or grocery stores with increasing interest of their health-promoting properties. For example, the consumption of broccoli has been associated with anti-cancer activity in in vitro and in vivo trails due to the high content of phytochemicals, minerals, vitamins, and fibers. Application of methyl jasmonate (MeJA) has been reported to enhance the potential health-promoting compounds in Brassica vegetables, glucosinolates (GS), especially indolyl GS neoglucobrassicin are induced by mimicking insect damage because MeJA activates herbivory defense system.

Although exogenous MeJA application has been recognized as a method to simulate insect herbivory and to invoke downstream defense mechanisms (inducing GS accumulation), only limited studies investigating the difference in metabolite changes or transcription level changes between two treatments in Brassica species. In Chapter 2, the metabolomic and transcriptomic changes between insect damage (4 days treatment of cabbage looper caterpillars) were compared. The primary result indicated levels of gene expression changes are slightly different between two treatments while the metabolite changes were similar but in different levels. For example, significantly increased indolyl GS was found in both treatments but higher in insect feeding groups.

GS loss during the cooking process has been intensively reported in Brassica vegetables; however, there is limited research on whether MeJA application affects GS retention after different cooking methods while MeJA application significantly increases inducible GS in Brassica vegetables. In Chapter 3, the phytochemical profile change after three different cooking methods (boiling, steaming, and microwaving) and two cooking times ( 2 and 5 minutes) on control and MeJA-treated broccoli. Among six cooking treatments (methods x times), 5 minutes boiling led to the most significant loss in total aliphatic (22\%) and indole GS (62\%) in control while it caused 47\% total aliphatic and 54\% indole GS loss in MeJA-treated broccoli; however, MeJA-treated broccoli contained 1.9-fold higher GS than the untreated broccoli after 5 min of boiling, which was considered the most drastic treatment in this study.

These changes by exogenous MeJA application, including GS level, GS hydrolysis products level, and primary metabolites, may alter the sensorial attributes of Brassica vegetables. Therefore, MeJA application may impact consumer acceptance and sensorial quality of Brassica vegetables, and the degree of impact may depend on level of myrosinase activation during cooking. In Chapter 4, an untrained consumer panel detected the differences between raw control and $250 \mu \mathrm{M}$ MeJA treated broccoli; however, four minutes of steaming eliminated the detectable bitterness in MeJA-treated broccoli and the consumer panel could not detect the difference between control and MeJA-treated broccoli. Neoglucobrassicin-derived hydrolysis products, $\mathrm{N}$ methoxyindole-3-carbinol, $N$-methoxyindolyl-3-carboxaldehyde, and $N$-methoxyindole-3acetonitrile, were the most important metabolites in determining the overall liking and the taste 
of broccoli samples using partial least square regression model. After 4 min steaming, MeJAtreated broccoli still contained 7.8-fold more neoglucobrassicin and 50\% more total GS than untreated broccoli

Despite all the positive results of exogenous MeJA application on Brassica vegetables, the applicability of this practice is limited without further evaluations on the feasibility from the consumers as well as from the growers. In Chapter 5, the consumer survey suggested that consumers who considered "anticancer broccoli" as the most attractive trait may be the potential customers who were willing to pay higher price ( $\$ 1.00$ or $\$ 1.50$ more per broccoli head) for MeJA-treated broccoli; however, recruited growers from the local farmer's market were concerned about the detectable bitterness in raw MeJA-treated broccoli would deter consumers from buying their broccoli in the future. Exogenous MeJA application may bring extra gross income for growers with small-scale broccoli production when we hypothesized a scenario from a very small farm ( $<1$ acre) when the elasticity of vegetable was set at -0.58 or- 0.79 .

Collectively, the results from this project implied that exogenous MeJA treatment (mimicking insect herbivory) on Brassica vegetables has its potential for use in the food industry and in the right market's niche. For example, MeJA-treated broccoli can be used as an ingredient to boost the nutrition quality and/or as a value-added ingredient in precooked meals because cooked MeJA-treated broccoli contained a higher level of GS comparing to cooked untreated broccoli. Therefore, exogenous MeJA application may be more suitable to the farms providing Brassica vegetables directly to the food processing industry instead of the farms selling fresh produce directly to the consumers. 


\section{ACKOWLEDGEMENTS}

It is a long journey to pursue a Ph.D. and it might be the most challenging decision I have made for myself.

First, I want to thank all my committee members (Dr. Ku, Dr. Waterland, Dr. Matak, Dr. Cumming, and Dr. Etienne) for the unconditional support for the past few years. This dissertation truly combined all the knowledge I have learned from you, and I cannot imagine completing the study without your help.

Second, I want to especially thank Dr. Verlinden for his support as a division director to support my degree. Without his help, there would have been more struggles on this road.

Third, I want to thank Dr. Shen for his help when I needed the most.

Last but not the least, I want to thank all of my peers that made this journey possible, especially my family and friends back in Taiwan, my partner Chun-Wei, and my good old friend Yu-Hung. Doing Ph.D. is a life-changing decision, and I am glad to have you for the past four years. 


\section{TABLE OF CONTENTS}

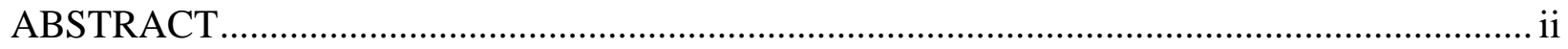

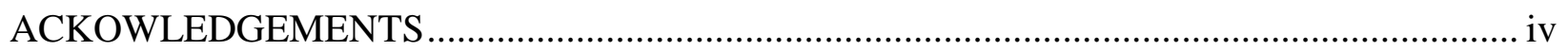

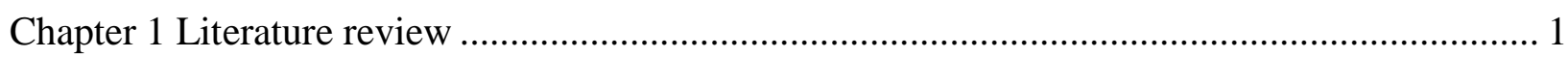

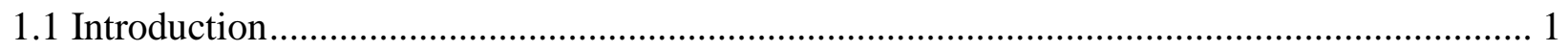

1.2 Mechanism and the role of MeJA as a plant regulator in Brassica vegetables ..................... 5

1.3 Advantage of exogenous MeJA application on Brassica vegetables .................................. 8

1.4 Disadvantage of MeJA application on Brassica vegetables - bitterness and enhanced ethylene

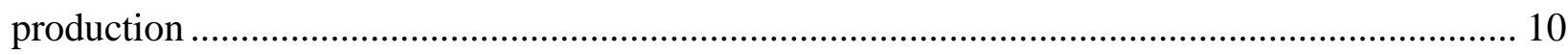

Chapter 2 Targeted metabolome and transcriptome analyses of 'Red Russian' kale (Brassicae napus var. pabularia) following methyl jasmonate treatment and larval infestation by the

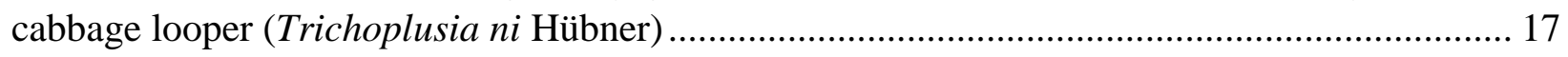

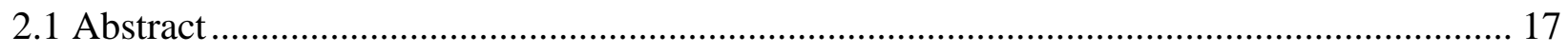

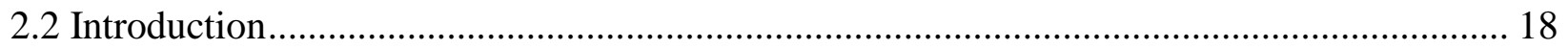

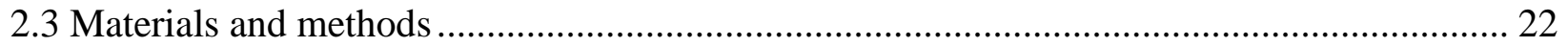

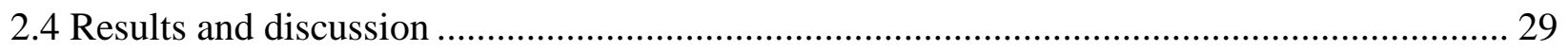

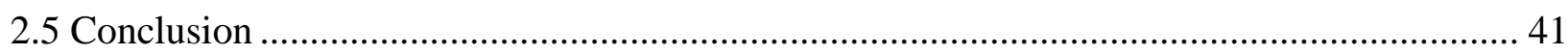

Chapter 3 Effect of methyl jasmonate treatment on glucosinolate retention and hydrolysis products after three cooking treatments ....................................................................... 50

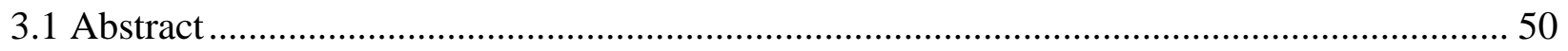

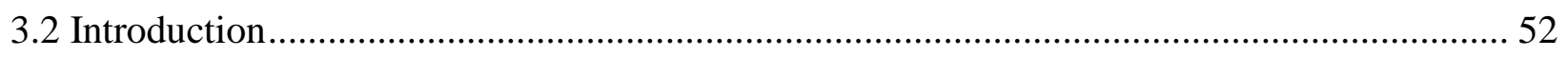

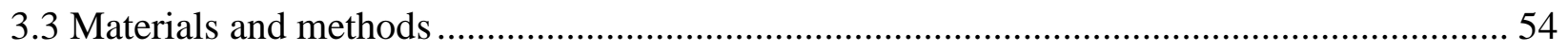

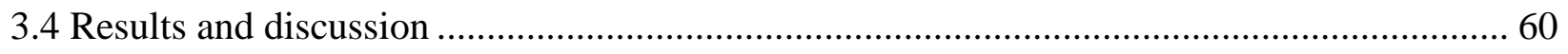

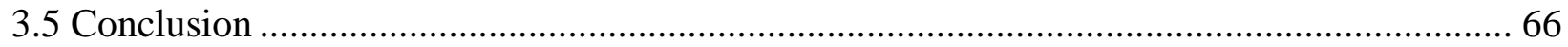

Chapter 4 Methyl jasmonate treated broccoli: impact on the production of glucosinolates and

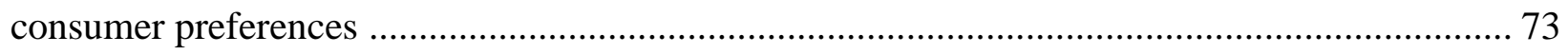

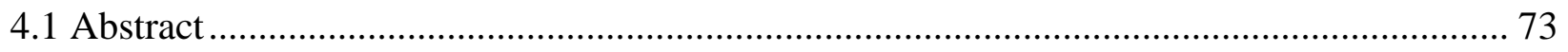

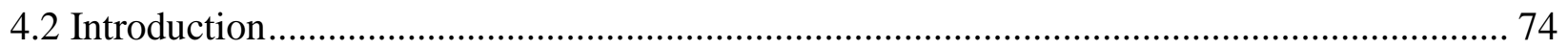

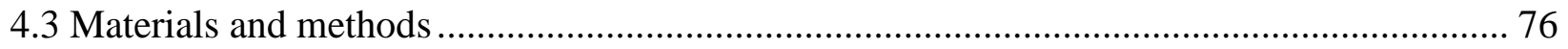

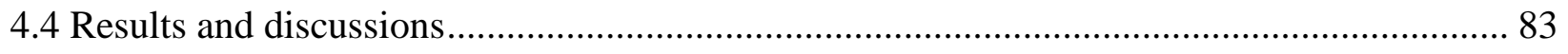

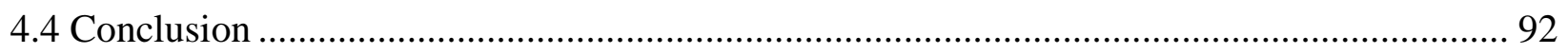

Chapter 5 Evaluating the feasibility of exogenous methyl jasmonate application on Brassica vegetables using consumer survey and producer focus group ........................................... 100

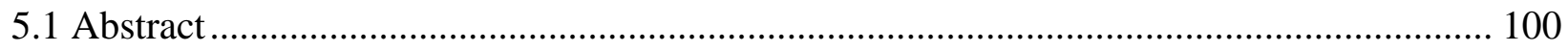




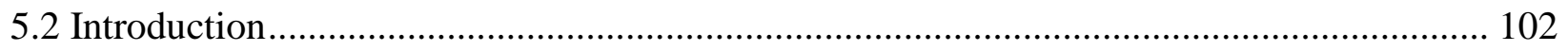

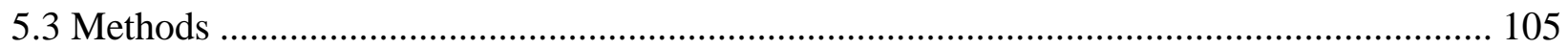

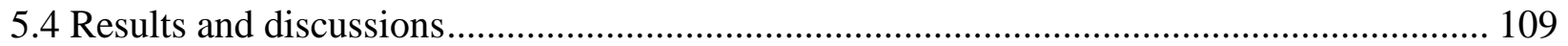

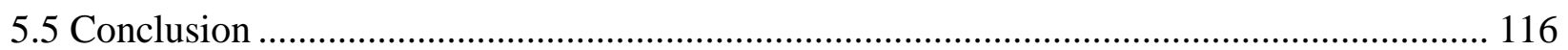

Chapter 6 Summary and discussion with future perspectives ...................................... 121

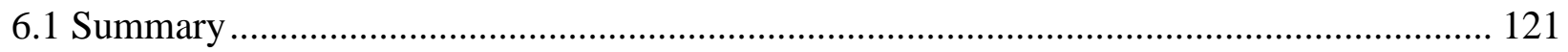

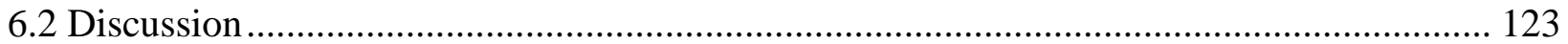

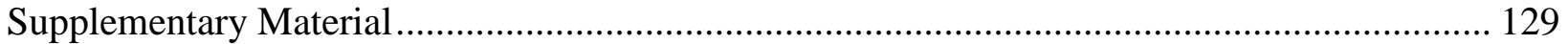

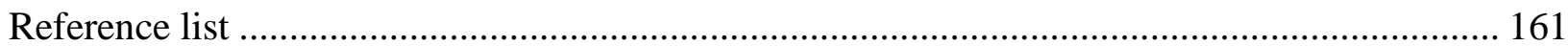




\section{LIST OF TABLES}

Table 2.1 Glucosinolate profiles ( $\mu \mathrm{mol} \cdot \mathrm{g}-1 \mathrm{DW})$ of 'Red Russian' controls and kale plants treated with $250 \mu \mathrm{M}$ methyl jasmonate (MeJA) or infested with cabbage looper for 4 days...... 44

Table 2.2 Glucosinolate hydrolysis profiles ( $\mu \mathrm{mol} \cdot \mathrm{g}-1 \mathrm{DW})$ of 'Red Russian' control kale and kale plants treated with $250 \mu \mathrm{M}$ methyl jasmonate (MeJA) or infested with T. ni for 4 days z. . 45

Table 3.1 Glucosinolate profile ( $\mu$ mole $\cdot \mathrm{g}^{-1} \mathrm{DW}$ ) in the cooking water collected from (A)2minute cooked broccoli and (B) 5-minute cooked broccoli with or without $250 \mu \mathrm{M}$ methyl jasmonate application (average $\pm \mathrm{SD}, \mathrm{n}=3$ ).

Table 3.2 Peak intensity $\left(\times 10^{3}\right)$ of neoglucobrassicin-derived hydrolysis products and total glucosinolate hydrolysis products of raw and cooked broccoli with or without $250 \mu \mathrm{M}$ methyl jasmonate application (average $\pm \mathrm{SD}, \mathrm{n}=3$ ).

Table 3.3 Fold changes of primary metabolites in MeJA-treated 'Green Magic' compared to untreated broccoli under the same cooking treatment.

Table 4.1. Glucosinolate profile $\left(\mu \mathrm{mole} \cdot \mathrm{g}^{-1} \mathrm{DW}\right)$ of raw and steamed broccoli with or without $250 \mu \mathrm{M}$ methyl jasmonate application (average $\pm \mathrm{SD}$ ).

Table 4 2. Volatile composition of raw and steamed 'Green Magic' broccoli with or without 250 $\mu \mathrm{M}$ methyl jasmoante treatment.

Table 4.3 Sensory attribute evaluation of raw and steamed broccoli with or without $250 \mu \mathrm{M}$ methyl jasmonate treatment. .....

Table 4.4. Metabolites that high in variable influence on projection (VIP) by partial least square

- regression model using overall liking score and taste/flavor score.

Table 5.1 Marginal effect of the ordered probit model for the extra premium price that consumers are willing to pay. 118

Table 5.2 Simulation of consumer demand using estimated elasticity 119 


\section{LIST OF FIGURES}

Figure 1.1 Precursor amino acids of glucosinolate and its breakdown (hydrolysis) products. Modified from Yan \& Chen, 2007.

Figure 1.2 Potential bitter phytochemicals in broccoli (italic font: potential compounds that have not being reported associating with bitterness in broccoli) 15

Figure 1.3 Overall scheme and objectives of this study 16

Figure 2.1 Expression of known genes involved in the GS biosynthesis pathway, myrosinase biosynthesis, and related to specifier proteins associated with GS hydrolysis in extracts of 'Red Russian' kale plants 4 days after treatment with $250 \mu \mathrm{M}$ MeJA or insect damage. 46

Figure 2.2 (A) Myrosinase activity (Unit/g DW) and (B) Nitrile formation (\%) of control, insectdamaged, and MeJA-treated kale.

Figure 2.3 Principle component analysis (A) score and (B) loading plots derived from nontargeted metabolite profiling of Brassica napus with the control plants, MeJA-treated plants, and insect-feeding plants.

Figure 2.4 Five biomarker metabolites selected with top-5 variable importance in projection (VIP) scores (VIP > 1.2) from all metabolites among control kales, MeJA-treated kales, and insect-infected kales.

Figure 3.1.Effect of cooking and $250 \mu \mathrm{M}$ MeJA treatment on (A) total aliphatic glucosinolates, (B) total indolyl glucosinolates, and (C) total glucosinolates in 'Green Magic' broccoli. 70

Figure 3.2 Effect of cooking and $250 \mu \mathrm{M}$ MeJA treatment on the retention rate of (A) total aliphatic glucosinolates (GS), (B) total indolyl GS, and (C) total GS.

Figure 3.3 Effect of cooking and $250 \mu \mathrm{M}$ MeJA treatment on myrosinase activity in broccoli... 72

Figure 4.1 GC chromatograph of glucosinolate hydrolysis products from control and MeJAtreated broccoli. (A) raw control broccoli, (B) raw MeJA-treated broccoli, (C) Steamed control broccoli, and (D) Steamed MeJA-treated broccoli.

Figure 4.2. Significant primary metabolites (A) amino acids, (B) organic acids, and (C) sugars and derivatives of raw and steamed 'Green Magic' broccoli with or without $250 \mu \mathrm{M} \mathrm{MeJA}$ treatment.

Figure 5.1 Distribution of consumers who were willing to pay more for the broccoli containing twice amount of the anti-cancer compounds $(n=48)$. 


\section{Chapter 1 Literature review}

\subsection{Introduction}

Jasmonic acid (JA) is a phytohormone secreted as part of the plant-insect herbivory defense system. It is a volatile compound that, when secreted in response to an insect attack, acts as a messenger to prime the neighboring plants for a potential assault (Howe \& Jander, 2008). A derivative of JA is methyl jasmonate (MeJA), which is also a volatile molecule that was first isolated from the plant Jasminum grandiflorum (Demole, Lederer, \& Mercier, 1962). Both JA and MeJA (and other derivatives) can be induced endogenously by insect attack and physical wounding; however, these compounds may also be applied exogenously in agriculture practice to improve postharvest quality by increasing fungi resistance of fresh fruits (Zhu \& Tian, 2012) and improving phytochemical profiles in multiple crops (Rohwer \& Erwin, 2008). MeJA is registered with Environmental Protection Agency (EPA) as a "generally recognized as safe" compound (FDA, 2013) with an acute oral median lethal dose $\left(\mathrm{LD}_{50}\right)$ greater than sodium chloride in rats (3129 mg/kg vs. $3000 \mathrm{mg} / \mathrm{kg}$, respectively). The EPA registration also reported that MeJA is non-toxic to human via oral exposure, dermal exposure, and inhalation exposure.

Exogenous MeJA can be applied to horticultural crops by dipping, vapor or liquid spray application both prior to or postharvest (Reyes-Díaz et al., 2016; Rohwer \& Erwin, 2008). This practice has been broadly tested on tomatoes, mangoes, and many Brassica vegetables including broccoli (Brassica oleraea var. italic), cauliflower (Brassica oleraea var. botrytis), and kale (Brassica oleracea or Brassica napus). In fruits, MeJA application has been shown to increase Botrytis cinerea resistance on tomatoes (Zhu \& Tian, 2012), improve volatile composition of tomatoes (Liu et al., 2018), and increase phenolic compounds and carotenoids of both tomatoes (Liu et al., 2018) and mangoes (Boonyaritthongchai, Chimvaree, Buanong, Uthairatanakij, \& 
Jitareerat, 2016). In Brassica vegetables, MeJA application can increase phytochemicals including flavonoids (quercetin and kaempferol) and glucosinolates (Ku, Jeffery, \& Juvik, 2014b).

In the 2017 annual vegetable summary by United States Department of Agriculture (USDA), area harvest and the value of utilized production of broccoli was the highest among all selected Brassica vegetables in the list (U.S. Department of Agriculture, 2017). In addition to the economic importance among all vegetables, the nutritional value of broccoli and other Brassica vegetables are also very high. Collard greens (Brassica oleracea var. acephala), mustard greens (Brassica juncea), kale (Brassica oleracea var. acephala or Brassicae napus var. pabularia), and Brussels sprouts (Brassica oleracea var. gemmifera) are considered "powerhouse vegetables" due to their high nutrition density (Di Noia, 2014). The consumption of Brassica vegetables is also associated with an anti-cancer and/or anti-inflammatory effect by cell culture essay, rat experiments, and in clinical trials (Becker \& Juvik, 2016). The high nutritional quality of Brassica vegetables comes from vitamin C, fiber, carotenoids, phenolics, and glucosinolates (GS).

GS are sulfur-containing compounds that present themselves almost exclusively in the Brassicales order, with one exception - the Putranjivaceae family (Malpighiales) (Cataldi et al., 2010). GS are defense compounds induced by endogenous or exogenous MeJA and can be derived from, and are typically classified by, their precursor amino acids. For example, the three major classes of GS are (1) aliphatic GS derived from alanine, leucine, isoleucine, valine, and methionine, (2) indolyl GS derived from tryptophan, and (3) arylaliphatic (or called aromatic or benzenic) GS derived from phenylalanine or tyrosine (Sønderby et al., 2010; 2017). 
Accumulation of GS in the plant can vary based on environmental factors (del Carmen Martínez-Ballesta, Moreno, \& Carvajal, 2013). Salinity, drought, elevated temperatures, light intensity, and light quality have been reported to regulate GS biosynthesis or the transcription factors in GS biosynthesis pathway. For instance, the accumulation of GS during stress condition is to maintain the turgor pressure and related with osmotic adjustment (Björkman et al., 2011; del Carmen Martínez-Ballesta, Moreno, \& Carvajal, 2013). Due to the presence of GS, many Brassica crops have been investigated in salinity-tolerant study (Ashraf \& McNeilly, 2004). Asides from being responsive to abiotic stress, GS are also critical specialized metabolites in response to the biotic stress. The absence of different GS is associated with decreased insect defense ability (Agerbirk et al., 2008; Beekwilder et al., 2008). Among all GS, indolyl GS was more sensitive to environmental change (Brown et al., 2002).

In agricultural practice, indolyl GS (glucobrassicin and neoglucobrassicin and an aromatic GS, gluconasturtiin, have been consistently reported to be increased by exogenous MeJA application (Ku et al., 2014b). GS are not bioactive until they are hydrolyzed to the corresponding breakdown products by the endogenous enzyme myrosinase (Figure 1.1). Myrosinase (thioglucoside glucohydrolase, EC 3.2.1.147) makes up a family of enzymes located in myrosin cells. When plants are attacked by insects or injured by physical wounding, the myrosin cell will rupture and myrosinase will hydrolyze GS to generate the corresponding breakdown products: isothiocyanates, nitriles, thiocyanates, epithionitriles, and oxazolidinethione. Among these, isothiocyanate (ITC) is the hydrolysis product most often reported to have high bioactivity. For example, the high biological activity of sulforaphane, an ITC derived from glucoraphanin (the major GS in broccoli), has been intensively tested in various kinds of assays (Becker \& Juvik, 2016). 
The hydrolysis catalyzed by myrosinase is dependent on other factors. For instance, the epithiospecifier protein (ESP) promotes the formation of epithionitriles, or nitriles, over ITC which might lead to the decrease of chemo-preventive properties of GS (Kupke et al., 2016). On the other hand, another cofactor of myrosinase, epithiospecifier modifier 1 (ESM1), was found to shift ITC formation over nitriles during the GS hydrolysis process (Burow et al., 2008; Zhang et al., 2006). In addition to the specifier protein, environmental factors such as temperature and $\mathrm{pH}$ (Hanschen et al., 2017; Vaughn and Berhow, 2005) will also affect the conversion preference of GS hydrolysis products.

Even though broccoli contains a wide variety of phytochemicals and has been linked to health benefits, consumers are sometimes deterred to purchase Brassica vegetables because of negative sensory experiences, such as bitter flavors or sulfuric off-odors. Sensory attributes are a major factor in a consumer's willingness to purchase vegetables and broccoli in particular (Costell et al., 2010; Drewnowski \& Gomez-Carneros, 2000); therefore, it is critical to understand the factors that influence the sensorial quality of broccoli. The bitterness of Brassica vegetables is often contributed to GS and their hydrolysis products (Figure 1.2). Results from an early study showed an inverse relationship between the sum of progoitrin and sinigrin and consumer's perception of "good taste” (Doorn et al., 1998). However, not all GSs are perceived as bitter or pungent and other secondary metabolites might contribute to the bitterness of broccoli as well. For example, flavonoids (a class of phenolic compounds) have also been perceived as being "bitter" by consumers in multiple studies with other crops (Drewnowski \& GomezCarneros, 2000; Fenwick et al., 1983; Wieczorek et al., 2017). 
To increase the understanding of exogenous MeJA application on edible crops and to evaluate the feasibility of exogenous MeJA on Brassica vegetables, following aspects will be examined (Figure 1.3):

(1) to compare the metabolism and gene expression between generalist insect (cabbage loopers) damage and exogenous MeJA application

(2) to determine if exogenous MeJA application affects GS loss during the cooking process

(3) to determine if exogenous MeJA application affects sensory quality

(4) to discover the targeted consumers for MeJA-treated Brassica vegetables

(5) to evaluate if exogenous MeJA application is profitable in small farm setting

\subsection{Mechanism and the role of MeJA as a plant regulator in Brassica vegetables}

The biosynthesis of JA and its mode of action has been extensively studied on the plant Arabidopsis and other Brassica vegetables (Ahuja et al., 2015; Howe \& Jander, 2008; Lortzing \& Steppuhn, 2016; Wasternack \& Hause, 2013). When JA biosynthesis is activated by insect herbivory or physical wounding, its precursor, linolenic acid, is released from galactolipids in the chloroplast membranes and is converted to JA. JA is further conjugated with isoleucine and forms JA-isoleucine (JA-Ile), which activates JA-related defense responses. Meanwhile, JA can be converted to MeJA as an airborne signal by carboxyl methyltransferase (Seo et al., 2001). Therefore, the major mechanism of exogenous MeJA application is to simulate the insect herbivory action. This method has been utilized to study the change of JA biosynthesis-related gene expression, GS gene expression, and the physiological role of JA as a phytohormone in multiple plants (Biondi, Scaramagli, Capitani, Maddalena Altamura, \& Torrigiani, 2001; Chen, Fei, Wang, Chen, \& Yan, 2015; Devoto et al., 2005). 
JA signaling and MeJA application will also activate a systemic acquired resistance (SAR), whereby if a plant is attacked by insects on one leaf, MeJA will be emitted from the wounded tissue into the air and will serve as an airborne signal to activate the defense response in undamaged leaves or other tissues (Lortzing \& Steppuhn, 2016). Therefore, MeJA application may be useful in agriculture practice because it does not need to be applied to the plant tissue harvest (i.e., broccoli florets). For example, when MeJA solution was sprayed onto broccoli leaves, the concentration of GS in the florets was similar to the florets where MeJA was applied on both the leaves and the florets (Ku et al., 2014b). In addition, exogenous application of MeJA can up-regulate the GS biosynthesis gene, related transcription factors, and myrosinase cofactors (Ku, Kim, Jeffery, Kang, \& Juvik, 2016; Yi et al., 2016, 2015).

The plant's response to exogenous MeJA application is more consistent than their response to actual insect herbivory. For example, a one-time application of $250 \mu \mathrm{M} \mathrm{MeJA}$ on plants prior to harvest consistently induce similar amount of GS regardless of environmental changes (Ku et al., 2013). Treatment of Brassica napus kale with $250 \mu \mathrm{M}$ MeJA four days prior to the harvest induced a higher accumulation of GS and consistently induced greater expression of GS biosynthesis genes when compared to generalist insect's attack (Chiu et al., 2018). On the other hand, cabbage looper infestation induced larger variation between replications, reduced myrosinase activity, and reduced nitrile formation (ratio between nitrile and ITC from the same precursor GS) in the same study. Therefore, the homogeneity of MeJA treatment makes this application more reproducible in terms of increasing nutritional value of Brassica vegetables by way of increasing the concentration of GS.

The crosstalk between JA and other plant hormones leads to the substantial change in plants. JA signaling pathway has been reported to crosstalk with auxin (Hentrich et al., 2013), 
gibberellin (Song, Qi, Wasternack, \& Xie, 2014), salicylic acid (Li, Han, Feng, Yuan, \& Huang, 2019; Thaler, Humphrey, \& Whiteman, 2012), abscisic acid (Ahmad et al., 2016; Lackman et al., 2011), and ethylene (Ahmad et al., 2016; Ellis, 2002; Li et al., 2019; Song et al., 2014). JA demonstrates the antagonism between gibberellin and salicylic acid in regulating seedling growth and in regulating pathogen-related proteins, respectively. These interactions can lead to a substantial change in crop physiology. For example, the interaction between JA and ethylene plays a critical role in apple fruit ripening and senescence (Lv, Ge, Li, Zhang, \& Li, 2017). MeJA application prior to harvest (Ku et al., 2013) or postharvest (Watanabe, Kamo, Nishikawa, \& Hyodo, 2000) was reported to enhance ethylene production in broccoli floret, which leads to the reduction of chlorophyll and the promotion of senescence. These changes can greatly affect the postharvest quality such as reduced storage time and visual appearance in broccoli (Ku et al., 2013).

Exogenous MeJA application may alter primary metabolites including amino acids, organic acids, and sugars (Kim, 2010). GS biosynthesis is part of JA signaling response. As glucose and a side chain derived from the precursor amino acid is the backbone of GS, when GS biosynthesis is up-regulated, the carbon source, i.e., glucose or amino acids, may be deprived. As JA responsiveness is part of the defense system, the trade-off between primary metabolism and secondary metabolism was speculated to optimize the fitness (Huot, Yao, Montgomery, \& $\mathrm{He}$, 2014), and thus plant growth is limited in this situation. It was suggested that a hidden transcriptional regulation or the crosstalk with other signaling pathways may be responsible for the reduced growth (Havko et al., 2016).

The shift between primary metabolism and secondary metabolism may also change the sensory quality. Sucrose, fructose, and glucose are the sweetest sugar (Moskowitz, 1970). Free 
amino acids in food was associated with sweetness (Bell, Methven, Signore, Oruna-Concha, \& Wagstaff, 2017). Therefore, it is important to consider the change of primary metabolites by exogenous MeJA application, as it may alter the sensory quality of Brassica vegetables.

\subsection{Advantage of exogenous MeJA application on Brassica vegetables}

According to the World Health Organization (WHO), one-third of cancers are associated poor lifestyle choices (i.e., sedentary, smoking, etc.) and diet choices, especially when diets are low in fruit and vegetable intake. Therefore, it is widely accepted that increased consumption of vegetables can significantly reduce the risk of cancer (Campbell et al., 1999). In clinical trials, diets that included broccoli (Ambrosone et al., 2004) and other Brassica vegetables (Atwell et al., 2015) had an inverse relationship with breast cancer risk and increased absorption of the chemopreventive compound sulforaphane. However, vegetable consumption in the US is substantially low; for example, in 2015, only $9.3 \%$ of the US adults between the ages $31-50$ met the recommended vegetable intake (Lee-Kwan, Moore, Blanck, Harris, \& Galuska, 2017). Increasing the concentration of health-promoting phytochemicals in vegetables is a promising strategy to improve the overall quality of the daily diet.

The greatest advantage of exogenous MeJA application in broccoli and other Brassica vegetables is the increased concentration of GS and other products of hydrolysis, regardless of seasonal variations, genotypes, and cultivation environment (greenhouse or in field conditions). Under greenhouse conditions, exogenous application of $250 \mu \mathrm{M}$ MeJA can increase neoglucobrassicin by 2 to 3-fold in broccoli cultivars 'Green Magic', 'Sultan', and 'Brigidier' (Kim \& Juvik, 2011). Under field conditions, a similar trend was also observed in broccoli cultivar 'Pirate', 'Expo’, 'Green Magic', ‘Imperial', and ‘Gypsy’ (Ku, Jeffery, \& Juvik, 2013). In kale and pak choi, there was a significant increase in neoglucobrassicin and its products of 
hydrolysis (Chiu, Juvik, \& Ku, 2018; Kim et al., 2017). It has also been shown that the application of $500 \mu \mathrm{M}$ MeJA will significantly increase the formation of sulforaphane without increasing its precursor GS, glucoraphanin, in broccoli (Ku, Jeffery, \& Juvik, 2013).

Neoglucobrassicin and its products of hydrolysis also demonstrated a significantly high correlation (0.7-0.9) with quinone reductase activity, which is an indicator of chemoprotective effect. Quinone reductase is the phase II detoxifying enzyme that can serve as a potential chemoprotective biomarker (Benson et al., 1980).

It is also noteworthy that the application of MeJA can sometimes increase phenolic compounds and anti-oxidant activity as well. The diversity of phenolic compounds among Brassica vegetables is large and its health-promoting effects were reported extensively (Cartea, Francisco, Soengas, \& Velasco, 2011). As little as $250 \mu \mathrm{M}$ MeJA application will significantly increase quercetin, a flavonoid, by 2-fold in apical kale leaves, although no effect was detected in broccoli (Ku \& Juvik, 2013). Another type of phenolic compound, phenolic acid, increased with MeJA treatment. For example, significant increases of phenolic acids (sinapic acid derivatives and chlorogenic acid derivatives) were observed in 10-day old red cabbage sprouts with seven days of $25 \mu \mathrm{M}$ MeJA application (Hassini et al., 2017).

Due to its role in plant herbivory defense, exogenous MeJA application also induced the defense ability against insects in broccoli. For example, when cabbage looper larvae was raised on MeJA-treated broccoli leaves, larval weight and survival rate five days after treatment was inversely correlated with the increased GS and its products of hydrolysis. This treatment also positively correlated with the number of days to pupation, which prolonged the window for pesticide application (Ku et al., 2016). 
Myrosinase hydrolyzes GS to its bioactive hydrolysis products; therefore, the myrosinase activity becomes a critical factor in determining the efficiency of GS hydrolysis. The concentration of MeJA application can significantly increase the myrosinase activity; when 500 $\mu \mathrm{M}$ MeJA application was applied, the myrosinase activity of broccoli floret was significantly increased by 58\% (Ku et al., 2013). On the other hand, when the application concentration was reduced to $250 \mu \mathrm{M}$, the effect of MeJA application was inconsistent between different species and different development stages (Ku, Jeffery, \& Juvik, 2014). For example, the application of $250 \mu \mathrm{M}$ MeJA did not have a significant effect on the myrosinase activity in 'Red Russian' kale (Chiu et al., 2018), 'Red Winter' kale (Ku et al., 2014), and different cultivars of pak choi (Kim et at., 2017).

\subsection{Disadvantage of MeJA application on Brassica vegetables - bitterness and enhanced ethylene production}

Sensorial quality dominates the consumer acceptance of Brassica vegetables (Zabaras et al., 2018). However, GS and the products of hydrolysis in Brassica vegetables were linked with perception of bitterness (Doorn et al., 1998; Engel, Baty, le Corre, Souchon, \& Martin, 2002a; Engel, Martin, \& Issanchou, 2006; Fenwick, Griffiths, \& Heaney, 1983). As mentioned in previous sections, exogenous MeJA application will increase certain GS and the products of hydrolysis. Understanding which GS are perceived as bitter is critical when determining the contribution of MeJA application to off-flavors of Brassica vegetables. Human perception of the bitter taste is a complex mechanism that involves more than three types of receptors (Drewnowski \& Gomez-Carneros, 2000). Two standard compounds, phenylthiocarbamide (PTC) and 6-n-propyl-2-thiouracil (PROP), are commonly used in experiments to determine or quantify the threshold of bitter perception. These compounds don't naturally occur in food; however, they 
have a common thiocyanate moiety $(N=C=S)$ that binds with bitter-taste receptors. People taste bitter when the thiocyanate moiety binds to the TAS2R receptor which is located on the surface of the taste cells of the tongue and personal sensitivity to bitterness is dependent on the different haplotypes of TAS2R receptors. TAS2R receptors can be categorized into supertasters (haplotype proline, alanine, valine (PAV)), non-tasters (haplotype alanine, valine, isoleucine (AVI), and heterozygous medium tasters (PAV/AVI) based on the change of amino acid sequence by the genetic single nucleotide polymorphism (SNP) (Wieczorek, Walczak, Skrzypczak-Zielińska, \& Jeleń, 2017). Aside from the TAS2R receptor, receptors TRPV1 and TRPA1 will also influence the sensorial properties of Brassica vegetables. Unlike the TAS2R receptor, TRPV1 receptor is activated by the specific covalent modification of cysteine side. The abovementioned receptors are located not only on the surface of the tongue, but also in respiratory epithelia, gastrointestinal tract, reproductive organs, and brain. Therefore, other sensation receptors such as olfactory receptors may be also involved in the bitter perception, too. However, the research on bitterness perception of human is not complete. Different ligands may bind to the same receptor and trigger the same sensorial perception while the chemical structure or specific modification mechanism might not yet be discovered.

The TAS2R and TRPA1 receptors provide one explanation of why GSL hydrolysis products ITCs are perceived as bitter; however, it does not provide the whole picture. Allyl ITC derived from sinigrin is one of the most common ITCs present in Brassica vegetables and it has the thiocyanate moiety on the structure. Allyl ITC will activate TRPA1 receptor and then generate the pungent taste (Zhang, 2010). The dominant ITC in broccoli is sulforaphane and its chemical structure also has thiocyanate moiety (see Table 1). However, the relationship between sulforaphane and its precursor GSL, glucoraphanin, on the perception of bitter flavor is unclear 
and reports are inconsistent (Bell et al., 2017). This may result from the difference in water solubility of sulforaphane and allyl ITC, as allyl ITC can more easily dissolve into water and trigger the bitter perception of TAS2R or TRPA1 when compared to sulforaphane. So far, most review or research articles on this topic focus on the correlation between sinigrin, progoitrin, glucobrassicin, neoglucobrassicin, and different ITCs and the bitterness perception (Table 1). Yet the research on the direct relationship using pure GS extraction from broccoli or GS isolation in sensory evaluation to determine the bitterness of single GS is relatively scarce (Bell, Oloyede, Lignou, Wagstaff, \& Methven, 2018).

The increased concentration of neoglucobrassicin by exogenous MeJA application may increase the bitterness of broccoli since neoglucobrassicin has been associated with bitter taste in cooked cauliflower using correlation analysis (Engel et al., 2002). Since the bitterness may affect consumer's willingness to consume or purchase Brassica vegetables (Cox et al., 2012;

Drewnowski \& Gomez-Carneros, 2000; Zabaras et al., 2018), the feasibility of MeJA may be limited.

The unpleasant flavor of broccoli includes the bitterness, pungency, astringency, and sometimes people even reported 'sulfur' odor as an unpleasant smell. The 'sulfur' and 'cabbagelike' smell usually comes from the organic sulfur volatiles, including dimethyl disulfide and dimethyl trisulfide. The application of MeJA will induce the emission of lipoxygenase (LOX) pathway volatiles and aldehydes (Chehab et al., 2008; Jiang, Ye, Li, \& Niinemets, 2017). Some aldehydes, such as hexanal, were found in the volatiles profile of broccoli (Wieczorek \& Jeleń, 2019). Therefore, MeJA application may change the volatile compound composition and further change the sensorial quality of broccoli. 
The application of MeJA has been associated with enhanced ethylene production and respiration. Along with the increased production of ethylene, it has been reported that postharvest application of $1 \mathrm{mM}$ MeJA (Watanabe et al., 2000) and preharvest $500 \mu \mathrm{M} \mathrm{MeJA}$ application (Ku et al., 2013a) will significantly increase the chlorophyll degradation in the first two and four days, respectively, after harvest when storing at $4{ }^{\circ} \mathrm{C}$. The degradation of chlorophyll is a cue to the plant senescence; therefore, the exogenous MeJA application might lead to the faster senescence of broccoli. Even though the visual greenness (hue angle) in the later study (Ku et al., 2013a) was not significantly observed, the chilling storage condition in the study might delay the visual effect. If the conditions of storage, transportation, or on-the-shelf of broccoli are not kept at $4^{\circ} \mathrm{C}$, the degradation of chlorophyll and ethylene production may accelerate and add to the negative impacts of MeJA application on broccoli (Wadhera \& Capaldi-Phillips, 2014). 


\section{Hydrolysis products}

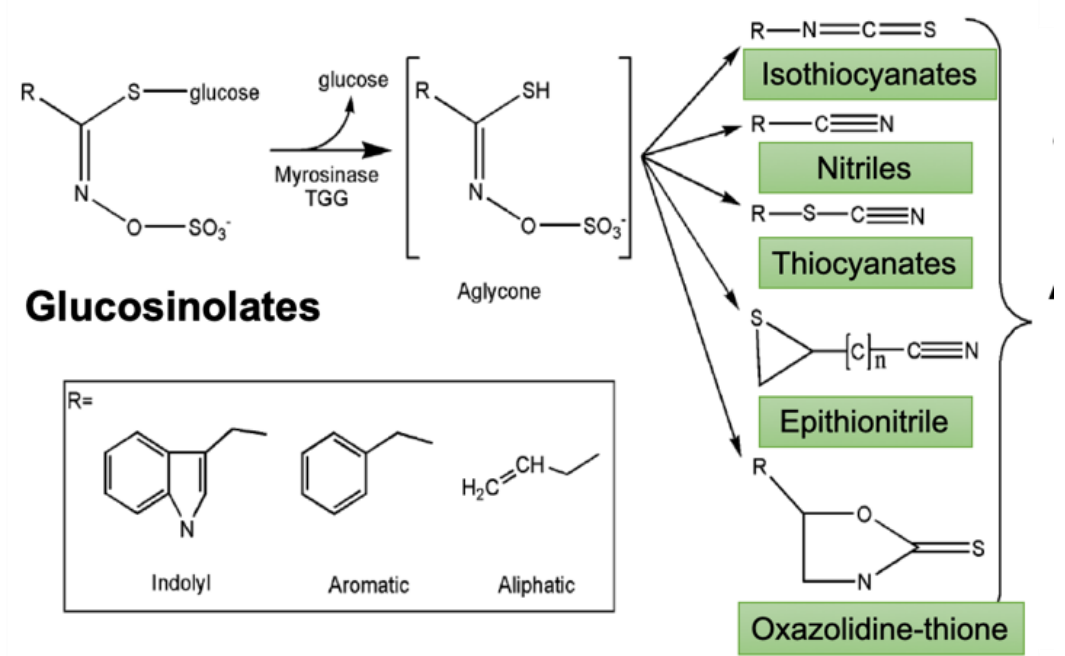

Figure 1.1 Precursor amino acids of glucosinolate and its breakdown (hydrolysis) products. Modified from Yan \& Chen, 2007. 


\begin{tabular}{|c|c|c|c|}
\hline \multicolumn{2}{|c|}{ Glucosinolate } & \multicolumn{2}{c|}{ Glucosinolate Hydrolysis products } \\
\hline Sinigrin & Progoitrin & Sulforaphane & Allyl isothiocyanate \\
\hline & & & \\
\hline
\end{tabular}

Figure 1.2 Potential bitter phytochemicals in broccoli (italic font: potential compounds that have not being reported associating with bitterness in broccoli) 


\section{Food supply chain}
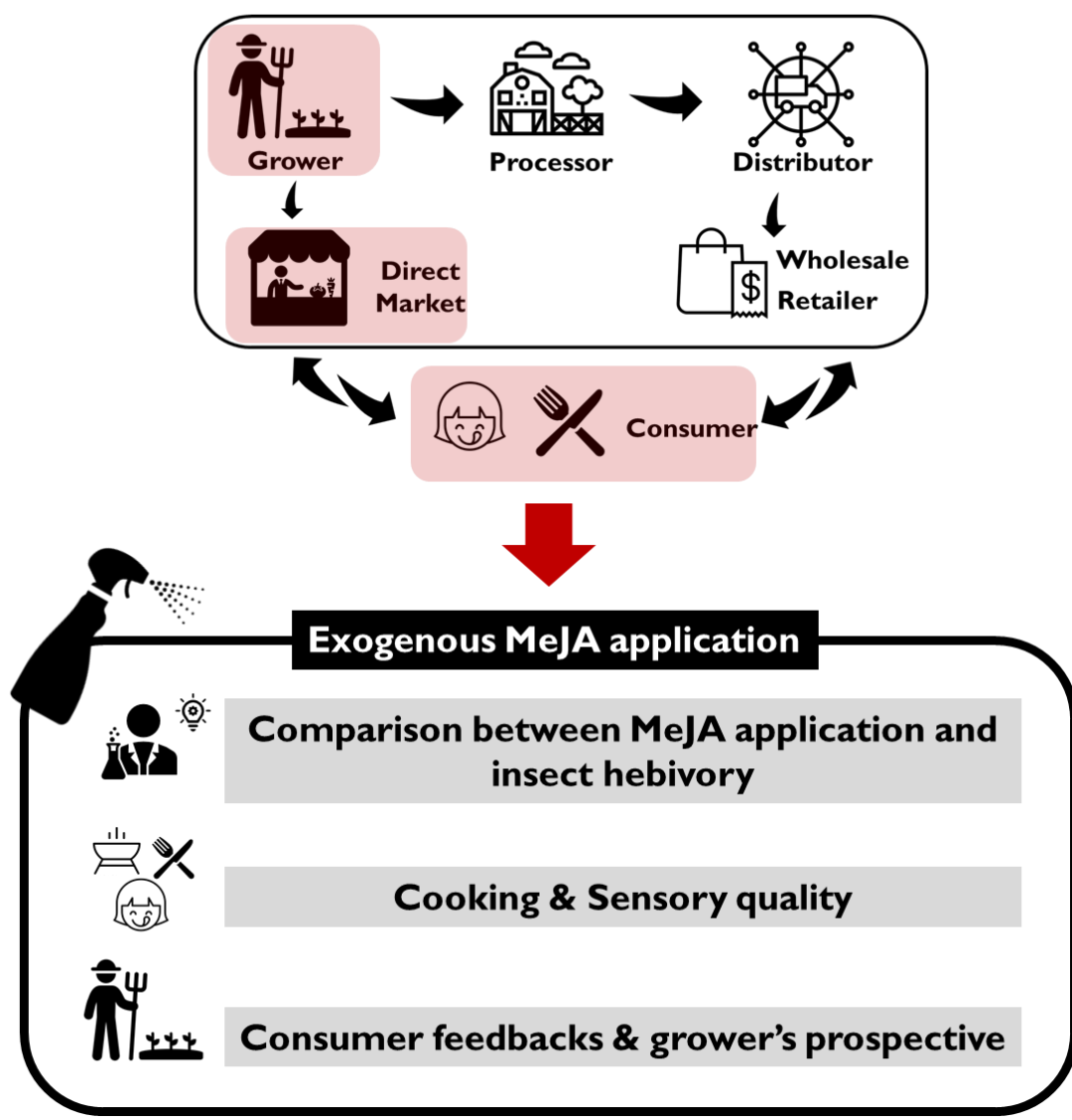

Figure 1.3 Overall scheme and objectives of this study 


\section{Chapter 2 Targeted metabolome and transcriptome analyses of 'Red Russian' kale (Brassicae napus var. pabularia) following methyl jasmonate treatment and larval infestation by the cabbage looper (Trichoplusia ni Hübner) ${ }^{1}$}

\subsection{Abstract}

Methyl jasmonate (MeJA), synthesized in the jasmonic acid (JA) pathway, has been found to upregulate glucosinolate (GS) biosynthesis in plant species of the Brassicaceae family. Exogenous application of MeJA has been shown to increase tissue GS concentrations and the formation of myrosinase-mediated GS hydrolysis products (GSHPs). In vitro and in vivo assays have demonstrated the potential health-promoting effects of certain GSHPs. MeJA is also known to elicit and induce genes associated with defense mechanisms to insect herbivory in Brassica species. To investigate the relationship between MeJA-induced GS biosynthesis and insect defense, three treatments were applied to "Red Russian" kale (Brassicae napus var. pabularia) seedlings: (1) a $250 \mu \mathrm{M}$ MeJA leaf spray treatment; (2) leaf infestation with larvae of the cabbage looper (Trichoplusia ni (Hübner)); (3) control treatment (neither larval infestation nor MeJA application). Samples of leaf tissue from the three treatments were then assayed for changes in GS and GSHP concentrations, GS gene biosynthesis expression, and myrosinase activity. Major differences were observed between the three treatments in the levels of GS accumulation and GS gene expression. The insect-damaged samples showed significantly lower aliphatic GS accumulation, while both MeJA and T. ni infestation treatments induced greater accumulation of indolyl GS. The gene expression levels of $C Y P 81 F 4, M Y B 34$, and $M Y B 122$ were significantly upregulated in samples treated with MeJA and insects compared to the control

\footnotetext{
${ }^{1}$ This study is published in International Journal of Molecular Sciences. Chiu, Y. C., Juvik, J., \& Ku, K. M. (2018). Targeted Metabolomic and Transcriptomic Analyses of 'Red Russian' Kale (Brassicae napus var. pabularia) Following Methyl Jasmonate Treatment and Larval Infestation by the Cabbage Looper (Trichoplusia ni Hübner). International journal of molecular sciences, 19(4), 1058.
} 
group, which explained the increased indolyl GS concentration. The results suggest that the metabolic changes promoted by MeJA application and the insect herbivory response share common mechanisms of induction. This work provides potentially useful information for kale pest control and nutritional quality.

\subsection{Introduction}

Kale is a widely consumed leafy vegetable grown around the world. Some kale cultivars are taxonomically classified as Brassica oleracea, while others are classified as Brassica napus. The popularity of kale has recently increased, as more information has been published regarding its high nutritional value (Migliozzi, Thavarajah, Thavarajah, \& Smith, 2015). As a member of Brassica genus, kale leaf tissue is rich in glucosinolates (GS) which are precursors of potential health-promoting compounds (Fahey et al., 2012; Fuentes, Paredes-Gonzalez, \& Kong, 2015; Ku et al., 2014a; Shapiro, Fahey, Wade, Stephenson, \& Talalay, 2001). Research results suggest that it is possible to improve GS biochemical profiles in kale production systems by changing cultivation practices and by genetic manipulation (Hahn, Müller, Kuhnert, \& Albach, 2016; Ku et al., 2014a; Velasco, Cartea, González, Vilar, \& Ordás, 2007). One method to potentially enhance GS accumulation in kale is through the exogenous application of the phytohormone methyl jasmonate (MeJA), similar to results observed in Arabidopsis and other Brassica crops including broccoli, cauliflower, Chinese cabbage, and pak choi (Chen et al., 2015; Kim \& Juvik, 2011; Ku, Choi, Kushad, Jeffery, \& Juvik, 2013; Ku et al., 2014a; Zang et al., 2016; Zang, Zheng, He, Hong, \& Zhu, 2015). Therefore, spray application of MeJA may be a practical and economical way to increase the nutritional quality of kale.

GS are sulfur- and nitrogen-containing plant secondary metabolites that are found in Brassicaceae species (Halkier \& Gershenzon, 2006; Sønderby, Geu-Flores, \& Halkier, 2010). 
GS can be categorized into three types, namely, aliphatic, indolyl, and aromatic GS on the basis of their respective biosynthetic precursor amino acid, i.e., methionine, tryptophan, and phenylalanine (Halkier \& Gershenzon, 2006). The bioactivities of GS are derived from its hydrolysis products formed by the action of the endogenous enzyme myrosinase. The hydrolysis of GS generates a wide array of GSHPs based on the the GS chemical structure, myrosinaseassociated protein cofactors, $\mathrm{pH}$, and the presence of $\mathrm{Mg}^{2+}$ and ascorbic acid (Auborn et al., 2003; Burow, Markert, Gershenzon, \& Wittstock, 2006; Fuentes et al., 2015; Halkier \& Gershenzon, 2006; Mohd Zul \& Surugau, 2016; Williams, Critchley, Pun, Nottingham, \& O'Hare, 2008; Zhang, Ober, \& Kliebenstein, 2006). GSHPs structure and concentrations have been associated with insect oviposition (Bruinsma, Van Dam, Van Loon, \& Dicke, 2007) and herbivore defense. In Arabidopsis, the hydrolysis products from two indolyl GS (4-methoxy glucobrassicin and neoglucobrassicin) have found to promote enhanced feeding-deterrent activity in aphids (Kim \& Jander, 2007).

GS in Brassicae species provides a mechanism of defense against insect herbivory and is partially regulated by the phytohormone jasmonic acid (JA). Aliphatic and indolyl GS concentrations were found to be positively correlated with insect defense (Barak, Liang, \& Narm, 2008; Beekwilder et al., 2008; Kim \& Jander, 2007). Indolyl GS biosynthesis and tissue concentrations were observed to be more sensitive to environmental variability and exogenous MeJA treatment (Brown et al., 2002; Ku et al., 2013). JA-upregulated genes induce a majority of herbivore resistance traits in Arabidopsis (Falk et al., 2014; Mao et al., 2017; Schuman \& Baldwin, 2016), including the biosynthesis of indolyl GSs (Kim \& Jander, 2007). It has been suggested that JA esterase converts MeJA into JA when exogenous MeJA is applied (Fürstenberg-Hägg, Zagrobelny, \& Bak, 2013). Greater concentrations of indolyl GS were 
observed to enhance insect deterrence. In 'Green Magic' and 'VI-158' broccoli cultivars, the survival rate and the larval weight of cabbage looper (Trichoplusia ni) has been found to be significantly reduced by a $400 \mu \mathrm{M}$ spray application of JA, attributable to the increase of neoglucobrassicin (indolyl GS) concentration (Ku, Becker, \& Juvik, 2016).

Cabbage looper is a lepidopteran insect and a major pest on cole crops across many regions in the United States (Greene, 1972). Cabbage looper is a generalist insect from the lepidopteran family Noctuidae, with different plant families, including Brassiceae, Solanaceae, and Cucurbitaceae, serving as hosts. As its name implies, cabbage looper is commonly observed on cabbage and other Brassica vegetables, such as broccoli. It is a major agricultural pest not only in the United States but also in Africa and in Asia ("HOSTS - A Database of the World's Lepidopteran Hostplants, Tak \& Isman, 2017). Because of their broad host range, cabbage loopers have evolved mechanisms to cope with different plant defense systems (Rivera-Vega, Galbraith, Grozinger, \& Felton, 2017). Therefore, this insect is an appropriate candidate to study insect herbivory in Brassica vegetables.

Insect herbivore-infested plants are challenged not only by physical damage: in fact, inter- and intra-cellular responses to wounding, insect oral secretions, oviposition fluids or peptides from insect's saliva (Fürstenberg-Hägg et al., 2013) promote the initiation of complex gene cascades associated with defense mechanisms. Cao's group has found that exogenous MeJA can partially compensate in the endogenous JA-deficient Arabidopsis mutant opr 3 by upregulating the biosynthesis of some GS but not of the aliphatic forms (Cao, $\mathrm{Li}$, Chen, $\mathrm{Liu}, \& \mathrm{Li}, 2016)$. In addition, jasmonate signaling is found to shape plant growth in Arabidopsis by modulating a transcriptional network (Campos et al., 2016) and the crosstalk with auxin signaling pathway (Huot, Yao, Montgomery, \& He, 2014). Normally, plants allocate energy directly toward growth 
or reproduction, unless their defense systems are induced. Herbivore-induced defense systems also include enhancing morphological forms of defense (trichrome production (Yuanyuan Zhang et al., 2015) or thickening cell walls (Houston, Tucker, Chowdhury, Shirley, \& Little, 2016)) to interfere with insect feeding. To synthesize defense compounds, plants need to utilize energy or precursor compounds including sugars and amino acids. Reduced sugar concentrations in response to MeJA treatment have been observed in Brassica oleracea and Brassica rapa (Kim, 2010; Kim et al., 2017). It also has been suggested that a hidden transcriptional network operates to reduce growth following JA signaling in lieu of depleting energy from carbon sources (Campos et al., 2016), thus influencing vegetable eating quality. In either situation, the activation of defense mechanisms may interfere with plant primary growth (McEwen, 2011).

Collectively, the application of MeJA sprays in agricultural production systems may result in similar physiological changes as observed under insect herbivore damage by increasing the concentration of different GS in different Brassica vegetables. However, the metabolic changes may not be identical to those observed under insect damage, because of temporal and spatial differences in the two scenarios. MeJA application is expected to be more homogenous than insect herbivory and to trigger a faster or more drastic metabolite change in plants. In contrast, insect herbivory may be a gradual and more complicated process, since the plants are simultaneously challenged by physical wounding and foreign peptides from the insect's saliva. Although previous research has focused on how GS affects insect herbivory in Arabidopsis mutant lines or other Brassica crops (Beekwilder et al., 2008; Kim \& Jander, 2007; Kliebenstein et al., 2001; Ku et al., 2016), how exogenously applied MeJA alters metabolite concentrations and how its effects differ from those of the actual insect herbivore on plants remain unclear. To determine the potential differences in the biochemical responses, in this study, we compare the 
changes in GS, GSHP, and primary metabolite concentrations in plants of "Red Russian" kale undergoing insect damage (leaf tissue 4 days after infestation of Trichoplusia ni larvae) or MeJA spray treatment (leaf tissue harvested 4 days after $250 \mu \mathrm{M}$ MeJA treatment).

\subsection{Materials and methods}

\subsubsection{Kale Cultivation and treatments}

The kale variety used in this experiment was 'Red Russian' (Johnny’s Selected Seeds, Winslow, ME). Seeds of 'Red Russian' kale were germinated in 32-cell plant plug trays filled with Sunshine ${ }^{\circledR}$ LC1 (Sun Gro Horticulture, Vancouver, BC, Canada) professional soil mix. The plants were grown in a greenhouse at the University of Illinois at Champaign-Urbana at $25^{\circ} \mathrm{C} / 18^{\circ} \mathrm{C}$ and $14 \mathrm{~h}-10 \mathrm{~h}$ day-night temperature regime, with supplemental high-intensity discharge (HID) lighting and 50-70\% relative humidity. Four weeks later, the plants in the vegetative growth stage were transferred to $1 \mathrm{~L}$ pots in the greenhouse under the same conditions. Kale plants with eight fully developed leaves were selected for the experiments.

The control plants were sprayed with a 0.1\% Triton X-100 (Sigma-Aldrich, St. Louis, MO, USA) solution. MeJA-treated plants were sprayed with a solution of $250 \mu \mathrm{M}$ JA (Sigma-Aldrich, St. Louis, MO, USA) with $0.1 \%$ Triton X-100. MeJA-treated kale leaf samples were harvested four days after spray treatment. Using a paintbrush, insect-treated plants were infested with second-instar larvae of $T$. $n i$ (Hübner). T. $n i$ was cultured in the Department of Entomology at the University of Illinois Urbana-Champaign. Six larvae of $T$. ni per plant were employed, with three plants per treatment, and the leaf tissue was scored for damage and then harvested for the analyses after four days of feeding.

\subsubsection{Quantification of Insect-Damaged Area Using ImageJ}


Image Processing and damaged leaf area calculation were carried out via an open-source software ImageJ (https://imagej.nih.gov/ij/). (Figure S1). Area selection was performed by "wand tool" or "freehand selection" to trace the desired area. Area calculation was based on the selected area and calculated by the software by extracting pixels information from a scale of the actual ruler in the taken photo.

\subsubsection{Quantification of Glucosinolate Concentrations}

All above-ground leaves of control, insect-infested, and MeJA-sprayed kale plants were harvested after four days of treatment for GS analysis. Above-ground aerial leaf and stem samples were frozen in liquid nitrogen and stored at $-20^{\circ} \mathrm{C}$ prior to freeze-drying. Freeze-dried tissues were ground into a fine powder using a coffee grinder and stored at $-20^{\circ} \mathrm{C}$ prior to GS analysis using high-performance liquid chromatography (HPLC). Extraction and quantification of GSs using HPLC were performed using a previously published method (Lisec, Schauer, Kopka, Willmitzer, \& Fernie, 2006). An amount of $200 \mathrm{mg}$ freeze-dried kale leaf and stem powder and $2 \mathrm{~mL}$ of $70 \%$ methanol were added to $10 \mathrm{~mL}$ tubes (Nalgene) and heated on a heating block at $95^{\circ} \mathrm{C}$ for $10 \mathrm{~min}$. After cooling on ice, $0.5 \mathrm{~mL}$ benzylglucosinolate $(1 \mathrm{mM})$ was added as an internal standard (POS Pilot Plant Corp, Saskatoon, SK, Canada), mixed, and centrifuged at $8000 \times g$ for $5 \mathrm{~min}$ at $4^{\circ} \mathrm{C}$. The supernatant was saved, and the pellet was reextracted with $2 \mathrm{~mL} 70 \%$ methanol at $95^{\circ} \mathrm{C}$ for $10 \mathrm{~min}$, after which the two extracts were combined. A subsample $(1 \mathrm{~mL})$ from each pooled extract was transferred into a $2 \mathrm{~mL}$ microcentrifuge tube (Fisher Scientific, Waltham, MA, USA). The proteins were precipitated with $0.15 \mathrm{~mL}$ of a 1:1 mixture of $1 \mathrm{M}$ lead acetate and $1 \mathrm{M}$ barium acetate. After centrifuging at $12,000 \times g$ for 1 min, each sample was loaded onto a column containing $1 \mathrm{M} \mathrm{NaOH}$ and $1 \mathrm{M}$ pyridine acetate-charged DEAE Sephadex A-25 resin (GE Healthcare, Piscataway, NJ, USA) for desulfation with arylsulfatase (Helix pomatia Type-1, Sigma-Aldrich, St. Louis, MO, USA) for 
$18 \mathrm{~h}$, and the desulfo-GSs were eluted with $3 \mathrm{~mL}$ Millipore-filtered ddH2O. The samples (100 $\mu \mathrm{L}$ ) were injected onto an Agilent 1100 HPLC system (Agilent, Santa Clara, CA, USA), equipped with a G1311A binary pump, a G1322A vacuum degasser, a G1316A thermostatic column compartment, a G1315B diode array detector, and an HP 1100 series G1313A autosampler. The UV detector was set at $229 \mathrm{~nm}$ wavelength. An all-guard cartridge pre-column (Alltech, Lexington, KY, USA) and a Kromasil RP-C18 column $(250 \mathrm{~mm} \times 4.6 \mathrm{~mm}, 5 \mu \mathrm{m}$ particle size, Supelco, Bellefonte, PA, USA) were used for quantification. The flow rate was 1 $\mathrm{mL} / \mathrm{min}$ with mobile phase A (water $w / 1 \%$ acetonitrile $v / v$ and $1 \mathrm{mM}$ ammonium acetate) and $\mathrm{B}$ ( $100 \%$ acetonitrile) with the following elution profile: $0 \min 0 \% \mathrm{~B}, 7 \mathrm{~min} 4 \% \mathrm{~B}, 20 \mathrm{~min} 20 \% \mathrm{~B}$, $35 \min 25 \% \mathrm{~B}, 36 \min 80 \% \mathrm{~B}, 40 \min 80 \% \mathrm{~B}, 41 \min 0 \% \mathrm{~B}$, and $50 \min 0 \% \mathrm{~B}$.

Benzylglucosinolate was used as an internal standard for quantification whose relative response factor (RRF) was set as 1 (Clarke, 2010). The UV response factors for various glucosinolates (ISO, 1992; Kusznierewicz, Iori, Piekarska, Namieśnik, \& Bartoszek, 2013; Wathelet, Marlier, Severin, Boenke, \& Wagstaffe, 1995) applied for quantification in this experiment were: glucoiberin 1.07, progoitrin 1.09, glucoraphanin 1.07, sinigrin 1.00, gluconapin 1.11, glucoerucin 1.00, glucobrassicin 0.29, 4-hydroxyglucobrassicin 0.28 , 4-methoxyglucobrassicin 0.25 , gluconasturtiin 0.95, and neoglucobrassicin 0.20 (Ku et al., 2016). RRF of 1hydroxyglucobrassicin was set as 0.28 , just like that of 4-hydroxyglucobrassicin because RRF was not available.

The identification of desulfo-GS was based on Capriotti et al. with a slight modification (Capriotti et al., 2018), and using fragmentation diagnostic ions from Kusznierewicz's group (Kusznierewicz et al., 2013) (Table S2), the profiles were validated by LC-tandem MS using a Waters 32 Q-Tof Ultima spectrometer coupled to a Thermo Accela 1200 UHPLC system 
coupled to a heated ESI source and to a Q Exactive high-resolution (HR) quadrupole and orbitrap LC-MS/MS (Thermo Scientific, Waltham, MA, USA), operated using full scan and a parallel reaction monitoring $(\mathrm{PRM})$ mode. A $250 \mathrm{~mm} \times 2.1 \mathrm{~mm}$ with internal diameter $5 \mu \mathrm{m}, 100$ $\AA$ Å, Kromasil RP-C18 column was used (AkzoNobel, Bohus, Sweden) for extract separation. Deionized distilled water with $0.1 \%$ formic acid (mobile phase A) and acetonitrile with $0.1 \%$ formic acid (mobile phase B) were used with a gradient system: 0 min 1.5\% B, 2 min 8\% B, 15 $\min 30 \%$ B, $16 \min 100 \%$ B, $25 \min 100 \%$ B, $25.1 \min 2 \%$ B, $30 \min 2 \%$ B, with a flow rate of $0.5 \mathrm{~mL} \mathrm{~min}^{-1}$. Ten microliters of desulfo-glucosinolate extract was injected. The column was heated to $40^{\circ} \mathrm{C}$ with a column heater. Mass identification was acquired in both positive and negative ion mode. Full scan mode range was $m / z$ 100.0-900.0. Nitrogen gas was used for ESI, collision Resolution was 70,000 FWHM (at $\mathrm{m} / \mathrm{z}$ 200). Automatic gas control (AGC) value was $3 \times 10^{6}$ in full scan; maximum ion inject time was $200 \mathrm{~ms}$. The ion spray voltage was $3.9 \mathrm{kV}$ with capillary temperature at $320^{\circ} \mathrm{C}$. Aux gas flow rate was S-Len, RF level was 55. MS/MS isolation window width was $4.0 \mathrm{~m} / \mathrm{z}$, with resolution at 17,500 in both positive and negative modes. AGC value was $2 \times 10^{5}$, and maximum ion inject time was $100 \mathrm{~ms}$.

\subsubsection{Quantification of Glucosinolate Hydrolysis Products}

Freeze-dried kale powder $(50 \mathrm{mg})$ was suspended in $1 \mathrm{~mL}$ distilled water in a $2 \mathrm{~mL}$ microcentrifuge tube (Fisher Scientific, Waltham, MA, USA). Hydrolysis products were generated naturally by endogenous myrosinase in the absence of light at room temperature for 24 h. After adding $1 \mathrm{~mL}$ of dichloromethane, the samples were centrifuged at $12,000 \times g$ for $2 \mathrm{~min}$, and the lower organic layer was collected. A gas chromatograph (Trace 1310 GC, Thermo Fisher Scientific, Waltham, MA, USA) coupled to an MS detector system (ISQ QD, Thermo Fisher Scientific, Waltham, MA, USA) and an autosampler (Triplus RSH, Thermo Fisher Scientific, Waltham, MA, USA). A capillary column (DB-5MS, Agilent Technologies, Santa Clara, CA, 
USA; $30 \mathrm{~m} \times 0.25 \mathrm{~mm} \times 0.25 \mu \mathrm{m}$ capillary column) was used to determine GS hydrolysis products. A $1 \mu \mathrm{L}$ sample of the dichloromethane extract was injected into the GC-MS with a split ratio of 1:1. After an initial temperature held at $40^{\circ} \mathrm{C}$ for $2 \mathrm{~min}$, the oven temperature was increased to $260^{\circ} \mathrm{C}$ at $10^{\circ} \mathrm{C} / \mathrm{min}$ and held for $10 \mathrm{~min}$ (Dosz, Ku, Juvik, \& Jeffery, 2014; Kim et al., 2017). The injector and detector temperatures were set to $200^{\circ} \mathrm{C}$ and $280^{\circ} \mathrm{C}$, respectively. The flow rate of the helium carrier gas was set to $1.1 \mathrm{~mL} / \mathrm{min}$. The peaks were identified using information from a previous publication (Kim et al., 2017) or by comparison with data in the National Institute of Standards and Technology (NIST) library.

\subsubsection{Measurement of myrosinase activities and nitrile formation}

Myrosinase activity was estimated as the total amount of hydrolysis products produced within $60 \mathrm{~min}$ (Dosz et al., 2014). One unit was defined as $1 \mu \mathrm{mol}$ of the above four hydrolysis products released per min. Nitrile formation in the sample was determined by incubating concentrated horseradish root extract with protein extracts of kale. The horseradish extract was used as an exogenous substrate source of sinigrin and gluconasturtiin at the saturated level to minimize the reaction of kale proteins with endogenous GS substrates. Subsequently, only the hydrolysis products from sinigrin and gluconasturtiin were the dominant compounds detected by GC-MS.

Freeze-dried kale powder $(75 \mathrm{mg})$ was mixed with $1.5 \mathrm{~mL}$ of concentrated "1091" horseradish root extract [10] in $2 \mathrm{~mL}$ microcentrifuge tubes (10 $\mathrm{g}$ of horseradish was mixed with $100 \mathrm{~mL}$ of $70 \%$ methanol. This solution was centrifuged at $4000 \times g$ for $5 \mathrm{~min}$. The supernatant of the horseradish root extracts was transferred to a beaker and boiled until the solvent was evaporated and then it was reconstituted with $50 \mathrm{~mL}$ of deionized water). After centrifugation at $12,000 \times \mathrm{g}$ for $2 \mathrm{~min}, 0.6 \mathrm{~mL}$ of supernatant was transferred to a $1.5 \mathrm{~mL}$ Teflon centrifuge tube (Savillex 
Corporation, Eden Prairie, MN, USA), and then $0.6 \mathrm{~mL}$ of dichloromethane was added. The tubes were placed upside down to minimize the loss of volatile compounds at room temperature for $10 \mathrm{~min}$. Then, the tubes were vortexed and centrifuged at $12,000 \times \mathrm{g}$ for $4 \mathrm{~min}$. The dichloromethane organic layer was injected into a GC-MS (Trace 1310 GC, Thermo Fisher Scientific, Waltham, MA, USA) coupled to an MS detector system (ISQ QD, Thermo Fisher Scientific, Waltham, MA, USA) and an autosampler (Triplus RSH, Thermo Fisher Scientific, Waltham, MA, USA). A capillary column (DB-5MS, Agilent Technologies, Santa Clara, CA, USA; $30 \mathrm{~m} \times 0.25 \mathrm{~mm} \times 0.25 \mu \mathrm{m}$ capillary column) was used to determine GS hydrolysis products. After an initial temperature held at $40^{\circ} \mathrm{C}$ for $2 \mathrm{~min}$, the oven temperature was increased to $320^{\circ} \mathrm{C}$ at $15^{\circ} \mathrm{C} / \mathrm{min}$ and held for $4 \mathrm{~min}$. Injector and detector temperatures were set to $270^{\circ} \mathrm{C}$ and $275^{\circ} \mathrm{C}$, respectively. The flow rate of the helium carrier gas was set to $1.2 \mathrm{~mL} / \mathrm{min}$. Standard curves of allyl isothiocyanate, 2-phenthyl isothiocyanate, and 3- phenylpropionitrile (SigmaAldrich, St Louis, MO, USA) were used for quantification. A standard curve generated from allyl isothiocyanate was applied to quantify of 1-cyano-2,3-epithiopropane.

\subsubsection{RNA extraction and quantitative real-time PCR}

Total RNA was isolated from control and insect- MeJA-treated freeze-dried kale powder samples using the RNeasy Mini Kit (QIAGEN), according to the manufacturer's instructions. The quantity of RNA was measured using a NanoDrop 3300 spectrophotometer (Thermo Scientific, Waltham, MA, USA). One $\mu$ g of total RNA was reverse-transcribed with Superscript ${ }^{\mathrm{TM}}$ III First-Strand Synthesis SuperMix for qRT-PCR (Invitrogen, Carlsbad, CA, USA), according to the manufacturer's instructions. The resulting cDNA samples were diluted to $1 / 10$ of their concentrations $(v / v)$ for qRT-PCR. The primer sets of GS biosynthesis genes, hydrolysis genes, and transcription factor genes were designed on the basis of database- 
published sequences (http://www.ocri-genomics.org/bolbase/index.html) (Hasperué, GómezLobato, Chaves, Civello, \& Martínez, 2013). A final list of the primers used, the gene model from which they were created, and a classification of the gene can be found in Table S3. The qRT-PCR data were expressed after normalization to the broccoli actin gene (BoACT1) [41]. The primers were synthesized by Integrated DNA Technologies (Coralville, IA, USA). Quantitative real-time PCR was carried out with the Power SYBR ${ }^{\circledR}$ Green RT-PCR Master Mix (QIAGEN) using an ABI 7900HT Fast Real-Time PCR System (Applied Biosystems, Foster city, CA, USA) according to the manufacturer's instructions. The relative expression ratio was determined with the equation $2^{-\Delta \Delta C \mathrm{t}}$, using the $B o A C T 1$ normalized $\Delta C_{\mathrm{t}}$ values generated by the $\mathrm{ABI} 7900 \mathrm{HT}$ Sequence Detection System Software 2.4 (Applied Biosystems) (Ku et al., 2013).

\subsubsection{Untargeted Metabolomics by GC-MS}

Primary metabolites were extracted by published protocol (Lisec et al., 2006) with modifications on the extraction solvent volume. The samples $(50 \mathrm{mg})$ were weighed in $2 \mathrm{~mL}$ microcentrifuge tubes, followed by addition of $80 \mu \mathrm{L}$ of ribitol $(10 \mathrm{mg} / \mathrm{mL})$ as an internal standard, then extracted with $1.4 \mathrm{~mL}$ of methanol at $75^{\circ} \mathrm{C}$. After cooling, the sample extracts were centrifuged at $15,000 \times g$ for $3 \mathrm{~min}$, and $0.7 \mathrm{~mL}$ of supernatants was transferred to new 2 $\mathrm{mL}$ microcentrifuge tubes. To fractionate polar compounds, $0.375 \mathrm{~mL}$ of cold chloroform $\left(-20^{\circ} \mathrm{C}\right)$ and $0.7 \mathrm{~mL}$ cold water $\left(4^{\circ} \mathrm{C}\right)$ were added. After vigorous mixing, the extracts were centrifuged at $15,000 \times g$ for $3 \mathrm{~min}$, and then $50 \mu \mathrm{L}$ supernatant was transferred to $1.5 \mathrm{~mL}$ microcentrifuge tubes. The extracts were dried using Vacufuge ${ }^{\mathrm{TM}}$ concentrator Eppendorf $^{\mathrm{TM}}$, Thermo Fisher Scientific, Waltham, MA, USA) with $10 \mu \mathrm{L}$ of methanol to facilitate water evaporation. The dried extracts were derivatized with $50 \mu \mathrm{L}$ of methoxyamine hydrochloride (40 $\mathrm{mg} / \mathrm{ml}$ in pyridine) for $90 \mathrm{~min}$ at $37^{\circ} \mathrm{C}$, then with $70 \mu \mathrm{L}$ MSTFA $+1 \% \mathrm{TMCS}$ at $37^{\circ} \mathrm{C}$ for $30 \mathrm{~min}$. 
The metabolites were analyzed using a GC-MS (Trace 1310 GC, Thermo Fisher Scientific, Waltham, MA, USA) coupled to an MS detector system (ISQ QD, Thermo Fisher Scientific, Waltham, MA, USA) and an autosampler (Triplus RSH, Thermo Fisher Scientific, Waltham, MA, USA). A capillary column (Rxi-5Sil MS, Restek, Bellefonte, PA, USA; $30 \mathrm{~m} \times 0.25 \mathrm{~mm} \times$ $0.25 \mu \mathrm{m}$ capillary column w/10 m Integra-Guard Column) was used to detect polar metabolites. After an initial temperature held at $80^{\circ} \mathrm{C}$ for $2 \mathrm{~min}$, the oven temperature was increased to $330^{\circ} \mathrm{C}$ at $15^{\circ} \mathrm{C} / \mathrm{min}$ and held for $5 \mathrm{~min}$. The injector and detector temperatures were set to $250^{\circ} \mathrm{C}$ and $250^{\circ} \mathrm{C}$, respectively. An aliquot of $1 \mu \mathrm{L}$ was injected with a split ratio of $70: 1$. The helium carrier gas was kept at a constant flow rate of $1.2 \mathrm{~mL} / \mathrm{min}$. The mass spectrometer was operated in positive electron impact mode (EI) at $70.0 \mathrm{eV}$ ionization energy at $\mathrm{m} / \mathrm{z} 40-500$ scan range.

The acquired chromatograms were converted to mzXML using the RawConverter (He, Diedrich, Chu, \& Yates, 2015). Peak detection and alignment were performed by XCMS package in R language with default settings (Smith, Want, O’Maille, Abagyan, \& Siuzdak, 2006). All data were normalized to unique ion peak (319) from the internal standard in the online platform MetaboAnalyst (Xia \& Wishart, 2002), and further statistical analysis was conducted after Pareto scaling. Metabolite identification was based on standard compounds (STD) in comparison with the mass spectra present in The National Institute of Standards and Technology (NIST) and retention times (Table S4).

\subsection{Results and discussion}

\subsubsection{Quantification of Insect-Damaged Area}

The damaged leaf area after 4 days in the presence of cabbage looper larvae (second instar) was calculated via the open-source software ImageJ tool (https://imagej.nih.gov/ij/). After 4 days 
of insect feeding, the damaged leaf area of 'Red Russian' kale from three individual plants was $12.2 \%, 5.1 \%$, and $15.0 \%$, respectively (average of $10.8 \%$; Figure S1). We observed significant variation in GS concentrations between the apical and basal leaves of kale (Ku et al., 2014a) indicating that indolyl GS concentration may be associated with cabbage looper's growth (Ku, Becker, et al., 2016). We quantified GS from different kale leaf locations from top to bottom (Table S1 and Figure S2). According to our Table S1, younger leaves had significantly higher GS than older leaves. Therefore, the variation in GS concentrations in the different feeding locations of larvae on kale plants might impact cabbage looper's activity, therefore producing differences in the extent of the damaged areas.

\subsubsection{Effect of MeJA Application and T. ni Treatment on GS, Their Hydrolysis Products, and Myrosinase Activity}

Four days after insect infestation and the application of $250 \mu \mathrm{M}$ MeJA treatment (Sigma,

St. Louis, MO, USA), treated and untreated kale plants were harvested, their leaf tissue was lyophilized, and the samples were stored at $-20^{\circ} \mathrm{C}$ for later analysis. A total of seven GS, including three aliphatic GS (progoitrin, glucoraphanin, and gluconapin), and four indolyl GS (glucobrassicin, neoglucobrassicin, 4-methoxyglucobrassicin, and 1-hydroxyglucobrassicin) were quantified by ultra-high performance liquid chromatography (UHPLC) and identified by LC mass spectrometer/mass spectrometer (Table 2.1; Table S2). Only trace amounts of glucoerucin and sinigrin were detected (data not shown). Kale seedlings subjected to cabbage looper feeding showed significantly lower accumulations $(25.2 \%, p<0.05)$ of total aliphatic GS (4.52 $\mu \mathrm{mol} / \mathrm{DW})$ than the control group $(6.04 \mu \mathrm{mol} / \mathrm{DW})$, while one-time application of $250 \mu \mathrm{M}$ MeJA showed no difference in total aliphatic GS (6.50 $\mu \mathrm{mol} / \mathrm{DW})$ compared to the control group 
(Table 2.1). MeJA application on kale in the present study was consistent with an earlier report according to which the application of MeJA had no effect on aliphatic GS concentrations in $B$. napus 'Red Winter' (Ku et al., 2014a). In the insect-damaged kale plants, the significantly lower concentration of aliphatic GS may result from the release of volatile hydrolysis products derived from aliphatic GS as a defense mechanism. Beekwilder and colleagues (Beekwilder et al., 2008) found that the larval weight of the generalist lepidopteran insect Mamestra brassicae was 2.6fold greater in an Arabidopsis mutant completely lacking aliphatic GS. A similar result was observed (Zhang et al., 2015) for cabbage looper larvae feeding on Arabidopsis. While the positive effects of aliphatic GS in the defense against generalist insects have been reported (Müller et al., 2010), the biosynthesis of aliphatic GS might not keep up with the loss of aliphatic GS volatile hydrolysis products. Previous work with Brassica oleracea has shown that variation of aliphatic GS is affected by genetic background, rather than by biotic and abiotic environmental factors (4.5\%) (Brown et al., 2002). Perhaps aliphatic GS biosynthesis in B. napus is regulated in a comparable manner, with limited response to biotic stress. This is in contrast to a previous study with Arabidopsis, where significantly higher accumulations of aliphatic GS were observed after a 2-day infestation with T. ni (Bidart-Bouzat \& Kliebenstein, 2011).

In the present study, the concentration of total indolyl GS was the greatest in insectdamaged kales (3.59 $\mu \mathrm{mol} / \mathrm{DW})$, followed by MeJA-treated kales $(2.80 \mu \mathrm{mol} / \mathrm{DW})$, and the control plants $(0.81 \mu \mathrm{mol} / \mathrm{DW})$. Indolyl GS biosynthesis has been found to be upregulated following MeJA spray applications (Ku, Becker, \& Juvik, 2016; Ku et al., 2014a). However, to our knowledge, the effect of $T$. $n i$ larval feeding on indolyl GS biosynthesis in plants has not been extensive studied. Three different responses to herbivory are recognized in plant defense systems: (1) herbivore-induce immunity (HTI) associated with oviposition, (2) herbivore- 
associated molecular patterns (HAMPs) and damage-associated molecular patterns (DAMPs), and (3) wound-induced resistance (WIR) by mechanical wounding (Wasternack \& Hause, 2013). A previous study (Bidart-Bouzat \& Kliebenstein, 2011) showed the T. ni feeding induced highlevel expression of jasmonic acid methyltransferase (at l g19640) and a significantly higher accumulation of indolyl GS in Arabidopsis. This implies that the plant response to T. $n i$ infestation and the activation of the JA signaling pathway are relatively similar to those observed upon exogenous MeJA application, since methyltransferase converts JA into MeJA, and exogenous MeJA application can restore defense responses in JA signaling-impaired plants (Bozorov, Dinh, \& Baldwin, 2017), and activate the JA signaling pathway as well. However, to plants, MeJA treatment may be less complex than insect damage treatment because mechanical wounding by insects and foreign peptides from the insect's saliva is lacking when applying exogenous MeJA. Considering this aspect, gene expression patterns altered by insect damage and by MeJA treatment may vary and thus lead to the difference in GS accumulation observed after the two treatments.

Myrosinase-mediated hydrolysis products, especially isothiocyanates from GS, have been found to interfere with the growth and development of generalist herbivores, while providing oviposition cues for specialists (Badenes-Perez, Gershenzon, \& Heckel, 2014; Hopkins, Dam, \& Loon, 2009; Tsao, Peterson, \& Coats, 2002). In this study, we detected seven hydrolysis products from aliphatic GS (sulforaphane, sulforaphane nitrile, 3-butenyl isothiocyanate, 1-cyano-3,4,epithiobutane, crambene, goitrin, and 1-cyano-2-hydroxy-3,4-epithiobutane) and three hydrolysis products from indolyl GS ( $N$-methoxyindolyl-3-carbinol (NMI3C), $N$-methoxyindolyl-3carboxyaldehyde (NM3CA), and indolyl-3-acetonitrile (I3A)) from kale leaf samples (Table 2.2). In most cases, the concentrations of the hydrolysis products showed no differences between 
the control and the treated samples, except for the concentration of 1-cyano-3,4,-epithiobutane, which was significantly lower in insect-damaged samples than in the control and MeJA-treated kale. Sulforaphane, goitrin, NMI3C, 1M3CA, and I3A were not detected in control plants. In insect-treated kales, GSHPs were mainly converted to isothiocyanate rather than to nitrile or epithionitrile forms, and this can be related to the function of GSHPs in plant herbivory defense function, which also may lead to differences between MeJA treatment and insect treatment. A previous study (Wittstock, Kliebenstein, Lambrix, Reichelt, \& Gershenzon, 2003) showed that isothiocyanates play a main role in plant defensive mechanism because of their universal toxicity; therefore, the higher accumulation of isothiocyanates in insect-damaged kales observed in the present study could be a strategy to repel $T$. $n i$ infestation.

The concentrations of the hydrolysis products across biological replicates of insectdamaged samples varied greatly, while the variation of the hydrolysis products among MeJAtreated plants remained small. The coefficient of variance (CV) across all 10 hydrolysis products from insect-damaged samples ranged from $34 \%$ to $136 \%$, while the CV of MeJA-treated samples was relatively small (10\% to $27 \%$ ). These results suggest that the stimulus from the insect herbivory process might be more dynamic than the one-time MeJA application, because insects can be exposed to significantly different levels of GS depending on kale leaf location and respond accordingly (Table $\mathrm{S} 1)$.

\subsubsection{Effect of MeJA application and T. ni larva feeding on the expression of genes related to} indolyl GS biosynthesis and of indolyl GS transcription factors, myrosinase, and specifier proteins

To understand the different effects of exogenous $250 \mu \mathrm{M}$ MeJA application and T. $n i$ larval feeding on the expression of genes related to GS biosynthesis or their hydrolysis products 
in 'Red Russian' kale, we investigated gene expression by quantitative real-time polymerase chain reaction (qRT-PCR) to measure the abundance of transcripts associated with the abovementioned pathways under the two treatments. We measured six genes involved in the aliphatic GS biosynthesis pathway, including MAM3, SOT17, SOT18, GSL-OH, MYB28, and MYB29, and nine genes involved in indolyl GS biosynthesis, including SUR1, CYP79B2, SOT16, CYP81F1, CYP81F2, CYP81F3, CYP81F4, MYB34, and MYB32 (Table 2.3; Table S3). In addition to the GS biosynthetic genes, genes involved in GS hydrolysis, including myrosinase-encoding genes $T G G 1$ and $T G G 2$, epithiospecifier protein (ESP), and epithiospecifier modifier 1 (ESM1), were measured to investigate the potential changes in the levels of hydrolysis products (Figure 2.1; Table S2).

Among these genes, exogenous $250 \mu \mathrm{M}$ MeJA application significantly upregulated the aliphatic GS-related genes MAM3 (chain elongation), SOT17 (core biosynthesis), SOT18 (core biosynthesis), GSL-OH (secondary modification), MYB29 (transcription factor), TGG1 and $T G G 2, E S P$, and $E S M 1$, between 1.7- and 9.4-fold. $T$. $n i$-induced damage only significantly upregulated SOT17, while there was a significant downregulation of MAM3, SOT17, SOT18, MYB28, and MYB29. These results indicated that a spray application of $250 \mu \mathrm{M}$ MeJA is sufficient to induce the majority of aliphatic GS biosynthesis genes in B. napus kale. It has been reported that a $400 \mu \mathrm{M}$ MeJA application to the heads of the broccoli cultivar 'Green Magic' can significantly increase SOT17, SOT18, TGG1, TGG2, and ESM1, but this is not observed in the doubled haploid inbred 'VI-158' (Ku, Becker, \& Juvik, 2016). Noticeably, the above genes were not significantly upregulated by a $200 \mu \mathrm{M}$ MeJA spray application in the previous study using 'Green Magic' and 'VI-158'. The effect of exogenous MeJA application on aliphatic GS gene expression varies depending on the application concentration and on plant genotype. MYB28 was 
the only gene that was significantly downregulated by the $250 \mu \mathrm{M}$ MeJA treatment. Different gene homologues encoding MYB28 may have differential sensitivity to exogenous MeJA, since it was reported that these genes can be differentially expressed in allopolyploid Brassica juncea (Augustine, Majee, Gershenzon, \& Bisht, 2013). Yi’s study (2016), that applied $250 \mu \mathrm{M}$ MeJA to B. oleracea kale, found that the relative gene expression of MYB28 was inconsistent among all measured homologues encoding MYB28. The authors reported that a $250 \mu \mathrm{M}$ MeJA application significantly increased, decreased, or did not change the levels of $M Y B 28$ transcripts. The transcript we measured was Bol036286, and our result agrees with their results regardless of the fact that different species were used in the two studies. Another possible reason is that the primers used in $B$. oleracea did not bind or effectively amplify the transcripts of $M Y B 28 . B$. napus kale is an allopolyploid that contains the genome from B. rapa (AA) and B. oleracea (CC) and many gene duplications, and subsequent functional divergence has occurred during polyploidization (Long et al., 2016, p. 28). Thus, MYB28 homologs might not have been accurately measured when we analyzed the data. Interestingly, even with increased gene expression levels, the total aliphatic GS concentration in MeJA-treated kale was not significantly greater compared to control kale samples (Table 2.1). This suggests that the gene expression patterns were not tightly associated with changes in aliphatic GS concentrations. In contrast, $T$. $n i$ larval feeding on 'Red Russian' kale did not upregulate aliphatic GS genes like MeJA treatment and significantly reduced the relative gene expression of MAM3, SOT17, SOT18, MYB28, and MYB29 (Table 2.3). The gene expression pattern in the $T$. $n i$-treated samples was associated with variations in aliphatic GS concentrations (Table 2.1), with reduced gene expression resulting in significant lower aliphatic GS concentrations. This suggests that the effect of four-day feeding of $T$. $n i$ on aliphatic GS gene expression in 'Red Russian' kale could 
be transient or that plant samples need to be harvested after fewer days of Please check if the original meaning is retained. Mewis et al. (Mewis et al., 2006) conducted an insect feeding study on the lepidopteran generalist Spodoptera exigua Hübner on Arabidopsis and reported MAM3 was significantly upregulated after only one day of larval feeding.

Indolyl GS and its hydrolysis products have been found to be strongly associated with insect herbivory (Fürstenberg-Hägg et al., 2013; Hopkins et al., 2009). In the present study, a $250 \mu \mathrm{M}$ MeJA application led to a significantly increased $(p<0.05)$ expression in 'Red Russian' kale of multiple genes related to indolyl GS biosynthesis, such as CYP79B2 (core biosynthesis), SOT16I (core biosynthesis), and CYP81F1, CYP81F2, CYP81F3, and CYP81F4 (side chain modification), and the transcription factors MYB34 and MYB122 (Table 3). Only the transcript levels of $C Y P 79 B 2, C Y P 81 F 4, M Y B 34$, and $M Y B 122$ were found to significantly increase in $T$. $n i$ larval-treated kale samples (Table 2.3).

MYB34 has been recognized as an activator in the indolyl GS biosynthesis pathway (Gigolashvili, Berger, \& Flügge, 2009) and after exogenous MeJA application in Arabidopsis (Dombrecht et al., 2007), pak choi (Brassica rapa ssp. chinensis) (Wiesner, Hanschen, Schreiner, Glatt, \& Zrenner, 2013), and two different broccoli cultivars (Brassica oleracea ssp. italica) (Ku, Becker, \& Juvik, 2016). Our findings are comparable to those of these previous studies where greater expression of MYB34 in MeJA-treated kale resulted in a significantly higher accumulation of indolyl GS (Table 2.1). CYP79B2 is an early upstream gene in the indolyl GS biosynthesis pathway that functions to convert tryptophan into indolyl-3acetaldoxime (Mikkelsen, Hansen, Wittstock, \& Halkier, 2000). This conversion is also the first step in the biosynthesis of the plant hormone auxin (Zhao et al., 2002). The interconnection between auxin homeostasis and the JA signaling pathway has been previously reported in 
Arabidopsis (Hentrich et al., 2013). The higher expression of CYP79B2 in MeJA-treated kale from the present study agrees with results observed in Arabidopsis. We also detected higher expression levels of CYP81F1, which is involved in indolyl GS side chain modification in MeJA-treated kale functions to convert glucobrassicin into 4-hydroxyindol-3-ylmethy GS. CYP81F1 has been reported to be induced by exogenous MeJA application in broccoli, kale, and cabbage (Yi et al., 2016). In Yi et al. (Yi et al., 2016), the significantly increased CYP81F1 expression levels resulting from a $250 \mu \mathrm{M}$ MeJA spray application to B. oleracea kale provided a result to similar ours. It is important to consider the difference in species response to exogenous MeJA application, which can be dose-dependent and cultivar-specific (Ku, Becker, et al., 2016). The higher expression levels of CYP81F1 may also be associated with the significantly greater accumulation of 4-methoxy glucobrassicin (Table 2.1). The gene expression of TGG2 and ESP in the MeJA-treated group showed a 5-fold and 9.5-fold significant increase when compared to the control kale. ESP is a myrosinase-associated protein involved in catalyzing the formation of epithionitriles or simple nitriles during GS hydrolysis, depending on the structure of GS (Bernardi, Negri, Ronchi, \& Palmieri, 2000). ESP promotes the formation of epithionitriles over other hydrolysis products, such as isothiocyanates, resulting in weaker human anti-cancer bioactivity involving the induction of phase II detoxification enzymes in Hepa1c1c7 cell cultures (Y Zhang, Talalay, Cho, \& Posner, 1992). Isothiocyanates are also the favored form of GSHPS that have insect repellent activity (Jeschke, Gershenzon, \& Vassão, 2016), and were associated with lower gene expression levels of ESP in $T$. ni-treated kale.

In the present study, insect-damaged kale did not show the same pattern of gene expression, suggesting that larval feeding was not perceived or did not provide as intense a response as observed following exogenous application of $250 \mu \mathrm{M}$ MeJA in kale plants. It is 
possible that the intensity of the response caused by six-second instar of $T$. ni feeding on 'Red Russian' kale plants was not strong enough to induce the expression of $C Y P 79 B 2$. However, considering the indolyl GS changes in Table 2.1, we suggest that the peak increase of gene expression in insect-damaged kale was too transient or that mRNA transcript turnover had reduced the transcripts' levels when the kale samples were harvested for the analyses $(\mathrm{Ku}$, Becker, \& Juvik, 2016; Mewis, Appel, Hom, Raina, \& Schultz, 2005; Mewis et al., 2006). T. ni damage may be more location-specific than MeJA treatment and, therefore, it might not be as powerful as MeJA application in triggering gene regulation. As a generalist insect, T. $n i$ is generally exposed to a broad array of plants. The chemical composition of $T$. $n i$ 's saliva can be altered when $T$. $n i$ confronts different plant species, as suggested by a recent study (Rivera-Vega et al., 2017). Therefore, it is likely that the damage by $T$. $n i$ was not as effective as the MeJA treatment in activating genes involved in the indolyl GS biosynthesis pathway. Noticeably, the expression levels of $M Y B 34, C Y P 79 B 2$, and $C Y P 81 F 1$ in insect-damaged were slightly higher than those in control kale, but not significantly different. Compared to the previous exogenous MeJA application study, significantly increased levels of gene expression were measured 2 days after MeJA application on broccoli (Ku et al., 2013) and pak choi (Wiesner et al., 2013), or three days after treatment on broccoli (Ku et al., 2016).

\subsubsection{Effect of MeJA application and T. ni larval feeding on myrosinase activity and nitrile} formation

The greatest myrosinase activity was detected in control samples $(4.86 \pm 0.16 \mathrm{Unit} / \mathrm{g}$ DW), while reductions in the activity were observed in both MeJA-treated samples $(3.42 \pm 1.00$ Unit/g DW) and insect-damaged samples $(2.63 \pm 1.31 \mathrm{Unit} / \mathrm{g}$ DW), with $30 \%$ and $45 \%$ lower activity than in the control, respectively (Figure $2.2 \mathrm{~A}$ ). In general, myrosinase activity can be 
influenced by insect herbivory; however, different responses related to myrosinase posttranslational glycosylation, myrosinase complexation with associated proteins, or the levels of the cofactor ascorbate were observed after treatment with specialist or generalist insects (Textor \& Gershenzon, 2009). Decreased myrosinase activity from insect-damaged kale in this study may result from the harvesting regime (four days after feeding initiation). Martin's group conducted a feeding experiment with the generalist insect Athalia rosae on Sinapis alba (Family Brassicaceae) and found a strong increase in myrosinase activity in day-one tissue samples, decreased activity in day-two samples, and no difference in control and insect-treated samples on day four after feeding initiation (Martin \& Müller, 2007). The effects of MeJA application on myrosinase activity differed among the species tested (broccoli or kales) and between leaf tissue samples at different developmental stages (apical or basal leaves). Additionally, a reduction of transcript abundance of myrosinase and its cofactors was observed in broccoli four days after $500 \mu \mathrm{mol} \mathrm{MeJA}$ application (Ku et al., 2013; Ku et al., 2014a). This suggests that there can be rapid changes in myrosinase activity in response to herbivory or MeJA application.

Nitrile formation (\%) from sinigrin was found to be increased only by MeJA treatment. Both MeJA spray application and larval feeding significantly decreased nitrile formation following hydrolysis of gluconasturtiin (Figure 2B). The concentration of nitriles formed indirectly reflects the activity of ESP which promotes epithionitriles as hydrolysis products from alkenyl GS (Zhang et al., 2006), or nitriles from other GS (Lambrix, Reichelt, Mitchell-Olds, Kliebenstein, \& Gershenzon, 2001). Isothiocyanates can be used as cues for host recognition by specialist herbivores. Generalist herbivores like $T . n i$ were found to show feeding preference for nitrile-producing Arabidopsis lines (Lambrix et al., 2001). Hydrolysis products from insectdamaged kale comprised lower concentrations of isothiocyanates. 


\subsubsection{Effect of MeJA Application and T. ni treatment on polar primary metabolites}

In addition to the change of secondary metabolites under the various treatments, changes in several primary metabolite concentrations (sugars, organic acid, sugar alcohol, and amino acids) were measured via gas chromatography-mass spectrometry (GC-MS) to further illustrate the effects of insect damage and MeJA treatment on kale plants.

Principal component analysis (PCA) was utilized to compare the changes in metabolites and identify significantly differences in metabolites between control and treatment groups. According to PCA scores plot, the distribution of metabolites in MeJA-treated and insectdamaged kale was clearly different compared to the control (Figure 2.3). Principal component 1 (PC1) mainly analyzed the treatment effects and accounted for $69.7 \%$ variation among the three treatments, whereas PC2 (14.9\%) accounted for variations of the biological replicates within each treatment. The loading plot shows that the variables are correlated with PC1 and PC2 (Figure 2.3).

The most significantly different concentrations in metabolites among the three treatments were selected by partial least-squares discriminant analysis (PLS-DA) using variable importance in projection (VIP) to estimate the importance of variables in the model. VIP score 1.2 was set as a threshold. Glucose, galactose, sucrose, fructose, and alanine (Figure 2.4) were above VIP score 1.2, which indicated that these compounds are important biomarkers that explain the concentration differences among treatments (Figure 2.4). Overall, sugar concentrations were decreased in both the MeJA and insect feeding treatments, suggesting that plants utilize monoand disaccharide sugars as a carbon source to synthesize GS or other chemicals for defense. Carbohydrates and amino acids are the primary metabolites that are reported to change under conditions of insect herbivory in plants. It has been reported that leaf sugar levels are regulated 
by JA signaling in Nicotiana attenuata plants at various developmental stages (Machado, Arce, Ferrieri, Baldwin, \& Erb, 2015). Mechado and colleagues (Machado et al., 2015) presented evidence that the concentrations of glucose, fructose, and sucrose in plants were inversely correlated with endogenous JA concentrations. Alanine was the only amino acid whose concentration differed among the treatment groups (VIP score $=1.4$ ). MeJA treatments have been reported to change the levels of sugars, organic acids, amino acids, and certain GSs in Brassica crops (H. S. Kim, 2010; Kim et al., 2017). The changes in these primary metabolites were found cutivar-specific in pak choi (Kim et al., 2017). Mono- and disaccharide sugar concentrations were decreased by MeJA spray treatment in 'Red Russian' kale, as previously reported (Kim et al., 2017). Sugars such as sucrose play a pivotal role in generating metabolic energy and provide a range of physiological functions in plant respiration (Fernie, Carrari, \& Sweetlove, 2004). The mono- and disaccharide sugar concentrations in insect-damaged kale were significantly higher than in MeJA-treated kale (Figure 2.4). This is the most notable difference in the metabolomes of MeJA-treated and insect-treated kale. Decreased photosynthetic activity in plants undergoing herbivory is common, since sources of carbon are needed to produce the defensive compounds. The jasmonic acid signaling pathway served to trigger these responses (Frier, Hernández, \& Tiessen, 2012; Havko et al., 2016). In this experiment, larvae-infested kale had a proportion of healthy leaves actively involved in photosynthesis.

\subsection{Conclusion}

This paper demonstrated how $250 \mathrm{uM}$ MeJA spray applications and T. ni larval feeding affected 'Red Russian' kale GS profiles, hydrolysis products formation, related gene expression profiles, and primary metabolites production. In general, both treatments significantly triggered 
the accumulation of GS. MeJA treatment induced higher accumulation of aliphatic GS, while the insect feeding treatment induced higher accumulation of indolyl GS (Table 2.1). Increased gene transcript abundance in the GS biosynthetic pathway was primarily observed in MeJA-treated kale (Table 2.3). Both treatments were shown to increase the hydrolysis products produced from the aliphatic or indolyl GS pathways compared to the control group (Table 2.2), but there was no distinguishable difference in terms of hydrolysis product concentrations between the MeJA application and the insect feeding treatment. Gene expression of myrosinase, the enzyme responsible for the hydrolysis of GS and the expression of transcripts of the specifier proteins ESP and ESM1 were significantly higher only in the MeJA-treated kale. Although MeJA-treated kale had slightly higher nitrile formation than control kale, insect damaged-kale had significantly lower nitrile formation than control kale. Therefore, with a better understanding of the regulation mechanism of ESP or ESM1 in the presence of insect damage, it could be useful to enhance isothiocyanate production from GS. We also detected differences in the concentrations of four sugars (glucose, galactose, sucrose, and fructose) and one amino acid (alanine) between control and treatment groups. This variation is likely due the production of defense-related compounds associated with insect herbivory and the induction of the jasmonic acid pathway. The ideal sample size was suggested to be 1:10 (Baggaley, 1983; Osborne \& Costello, 2004) for PCA, so undoubtedly the statistical power was not ideal because of the small sample size. Yet, this study still suggests the potential utility of MeJA application in 'Red Russian' kale to prime plants for enhanced insect defense by eliciting greater accumulations of GS and of its hydrolysis products. This study also suggests that MeJA spray treatment potentially enhances the health-promoting properties of kale by increasing the concentrations of GS and GS hydrolysis products (Fuentes et al., 2015). 
Table 2.1 Glucosinolate profiles ( $\mu$ mole.g-1 DW) of 'Red Russian' controls and kale plants treated with $250 \mu \mathrm{M}$ methyl jasmonate (MeJA) or infested with cabbage looper for 4 days.

\begin{tabular}{|c|c|c|c|c|c|c|c|c|c|c|}
\hline Samples & $\begin{array}{l}\text { Gluco- } \\
\text { raphanin }\end{array}$ & $\begin{array}{l}\text { Gluco- } \\
\text { napin }\end{array}$ & Progoitrin & $\begin{array}{l}\text { Gluco- } \\
\text { brassicin }\end{array}$ & $\begin{array}{l}\text { Neo- } \\
\text { glucobrass } \\
\text { icin }\end{array}$ & $\begin{array}{l}\text { 4- } \\
\text { Methoxy- } \\
\text { glucobrass } \\
\text { icin }\end{array}$ & $\begin{array}{l}1- \\
\text { Hydroxy- } \\
\text { glucobrass } \\
\text { icin }\end{array}$ & $\begin{array}{l}\text { Total } \\
\text { Aliphatic } \\
\text { GS }^{\text {z }}\end{array}$ & $\begin{array}{l}\text { Total } \\
\text { Indolyl } \\
\text { GS }\end{array}$ & Total GS \\
\hline Control & $\begin{array}{c}1.14 \pm 0.16 \\
\mathrm{y} a\end{array}$ & $\begin{array}{c}0.64 \pm 0.02 \\
\mathrm{a} \\
\end{array}$ & $\begin{array}{c}4.26 \pm \\
0.55 \mathrm{ab} \\
\end{array}$ & $\begin{array}{c}0.31 \pm \\
0.07 \mathrm{c}\end{array}$ & $\begin{array}{l}0.31 \pm \\
0.07 \mathrm{~b}\end{array}$ & $\begin{array}{l}0.03 \pm \\
0.01 \mathrm{c}\end{array}$ & $\begin{array}{l}0.17 \pm \\
0.02 \mathrm{a}\end{array}$ & $\begin{array}{l}6.04 \pm \\
0.40 \mathrm{a}\end{array}$ & $\begin{array}{l}0.81 \pm \\
0.17 \mathrm{c}\end{array}$ & $\begin{array}{l}6.85 \pm \\
0.44 \mathrm{~b}\end{array}$ \\
\hline МeJA & $\begin{array}{c}1.13 \pm 0.20 \\
\mathrm{a}\end{array}$ & $\begin{array}{c}0.67 \pm 0.09 \\
\mathrm{a}\end{array}$ & $\begin{array}{l}4.71 \pm \\
0.40 \mathrm{a}\end{array}$ & $\begin{array}{c}0.98 \pm \\
0.15 b\end{array}$ & $\begin{array}{l}1.65 \pm \\
0.19 \mathrm{a}\end{array}$ & $\begin{array}{c}0.06 \pm \\
0.01 \mathrm{~b}\end{array}$ & $\begin{array}{l}0.10 \pm \\
0.03 \mathrm{~b}\end{array}$ & $\begin{array}{l}6.50 \pm \\
0.60 \mathrm{a}\end{array}$ & $\begin{array}{l}2.80 \pm \\
0.31 \mathrm{~b}\end{array}$ & $\begin{array}{l}9.31 \pm \\
0.85 \mathrm{a}\end{array}$ \\
\hline $\begin{array}{l}\text { Insect } \\
\text { damage }\end{array}$ & $\begin{array}{c}0.77 \pm 0.14 \\
b\end{array}$ & $\begin{array}{c}0.30 \pm 0.09 \\
b\end{array}$ & $\begin{array}{l}3.44 \pm \\
0.86 \mathrm{~b}\end{array}$ & $\begin{array}{l}1.68 \pm \\
0.31 \mathrm{a}\end{array}$ & $\begin{array}{l}1.73 \pm \\
0.17 \mathrm{a}\end{array}$ & $\begin{array}{l}0.09 \pm \\
0.02 \mathrm{a}\end{array}$ & $\begin{array}{c}0.12 \pm \\
0.04 \mathrm{ab}\end{array}$ & $\begin{array}{l}4.52 \pm \\
1.04 \mathrm{~b}\end{array}$ & $\begin{array}{l}3.59 \pm \\
0.46 \mathrm{a}\end{array}$ & $\begin{array}{l}8.11 \pm \\
1.35 \mathrm{ab}\end{array}$ \\
\hline
\end{tabular}

For a given glucosinolate, mean values within the same column followed by the same letter are not significantly different by Student's significance test $(p<0.05)$.

${ }^{\mathrm{z}} \mathrm{GS}=$ glucosinolate.

${ }^{\mathrm{y}}$ Values are means of three replications. 
Table 2.2 Glucosinolate hydrolysis profiles ( $\mu$ mole.g-1 DW) of 'Red Russian' control kale and kale plants treated with $250 \mu \mathrm{M}$ methyl jasmonate (MeJA) or infested with T. ni for 4 days z.

\begin{tabular}{|c|c|c|c|c|c|c|c|c|c|c|}
\hline Samples & $\begin{array}{l}\text { Sul- } \\
\text { foraphane }\end{array}$ & $\begin{array}{l}\text { Sul- } \\
\text { foraphane } \\
\text { Nitrile }\end{array}$ & $\begin{array}{l}3 \text {-butenyl } \\
\text { isothiocya } \\
\text { nate }\end{array}$ & $\begin{array}{l}\text { 1-cyano- } \\
3,4- \\
\text { epithiobut } \\
\text { ane }\end{array}$ & Crambene & Goitrin & $\begin{array}{l}\text { 1-cyano- } \\
2- \\
\text { hydroxy- } \\
3,4- \\
\text { epithiobut } \\
\text { ane }\end{array}$ & I3A & NMI3C & NM3CA \\
\hline Control & 0 & $\begin{array}{l}0.08 \pm \\
0.02 \mathrm{a}\end{array}$ & $\begin{array}{l}0.13 \pm \\
0.04 \mathrm{a}\end{array}$ & $\begin{array}{l}1.11 \pm \\
0.12 \mathrm{a}\end{array}$ & $\begin{array}{l}1.48 \pm \\
0.19 \mathrm{a}\end{array}$ & 0 & $\begin{array}{l}1.30 \pm \\
0.24 \mathrm{a}\end{array}$ & 0 & 0 & 0 \\
\hline MeJA & $\begin{array}{l}0.03 \pm \\
0.01 \mathrm{a}\end{array}$ & $\begin{array}{l}0.11 \pm \\
0.02 \mathrm{a}\end{array}$ & $\begin{array}{l}0.38 \pm \\
0.04 \mathrm{a}\end{array}$ & $\begin{array}{l}1.37 \pm \\
0.28 \mathrm{a}\end{array}$ & $\begin{array}{l}1.94 \pm \\
0.14 \mathrm{a}\end{array}$ & $\begin{array}{l}0.07 \pm \\
0.01 \mathrm{a}\end{array}$ & $\begin{array}{l}1.70 \pm \\
0.21 \mathrm{a}\end{array}$ & $\begin{array}{l}0.08 \pm \\
0.02 \mathrm{a}\end{array}$ & $\begin{array}{l}0.37 \pm \\
0.04 \mathrm{a}\end{array}$ & $\begin{array}{c}0.05 \pm \\
0.01 \mathrm{a}\end{array}$ \\
\hline $\begin{array}{l}\text { Insect } \\
\text { damage }\end{array}$ & $\begin{array}{l}0.19 \pm \\
0.23 \mathrm{a}\end{array}$ & $\begin{array}{l}0.08 \pm \\
0.03 \mathrm{a}\end{array}$ & $\begin{array}{l}0.75 \pm \\
0.72 \mathrm{a}\end{array}$ & $\begin{array}{l}0.56 \pm \\
0.28 \mathrm{~b}\end{array}$ & $\begin{array}{l}1.67 \pm \\
0.58 \mathrm{a}\end{array}$ & $\begin{array}{l}0.42 \pm \\
0.57 \mathrm{a}\end{array}$ & $\begin{array}{l}1.28 \pm \\
0.57 \mathrm{a}\end{array}$ & $\begin{array}{l}0.08 \pm \\
0.03 \mathrm{a}\end{array}$ & $\begin{array}{l}0.65 \pm \\
0.35 \mathrm{a}\end{array}$ & $\begin{array}{l}0.05 \pm \\
0.03 \mathrm{a}\end{array}$ \\
\hline
\end{tabular}

Sulforaphane, isothiocyanate of glucoraphanin; 3-butenyl isothiocyanate, isothiocyanate of gluconapin; 1-cyano-3,4,epithiobutane, epithioitrile from gluconapin; crambene, nitrile from progoitrin; goitrin, oxazalidine from progoitrin; 1-cyano-2hydroxy-3,4-epithiobutane, epithioitrile from progoitrin; I3A, indolyl-3-acetonitrile, nitrile from glucobrassicin; NMI3C, $N$ methoxyindolyl-3-carbinol from neoglucobrassicin; NM3CA, $N$-methoxyindolyl-3-carboxaldehyde from neoglucobrassicin. For a given hydrolysis product, mean values within the same column followed by the same letter are not significantly different by Student's significance test $(p<0.05) .{ }^{\mathrm{z}}$ Values are means of three replications. 


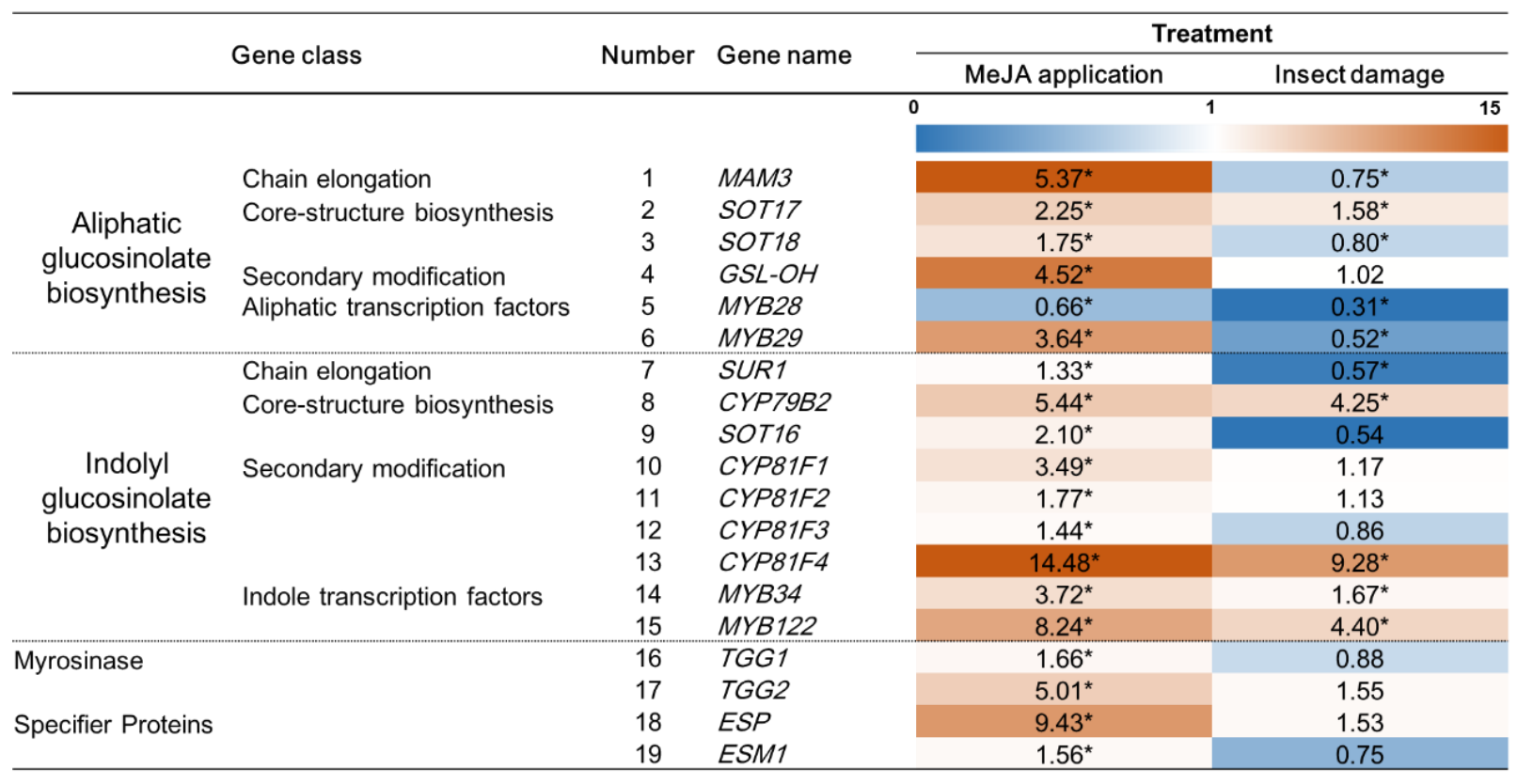

Figure 2.1 Expression of known genes involved in the GS biosynthesis pathway, myrosinase biosynthesis, and related to specifier proteins associated with GS hydrolysis in extracts of 'Red Russian' kale plants 4 days after treatment with $250 \mu \mathrm{M}$ MeJA or insect damage. Asterisk indicates a significant difference compared to the control using Student's $t$-test ( $p<$ 0.05). The values highlighted in red and in blue indicate significantly upregulated or downregulated, respectively. 


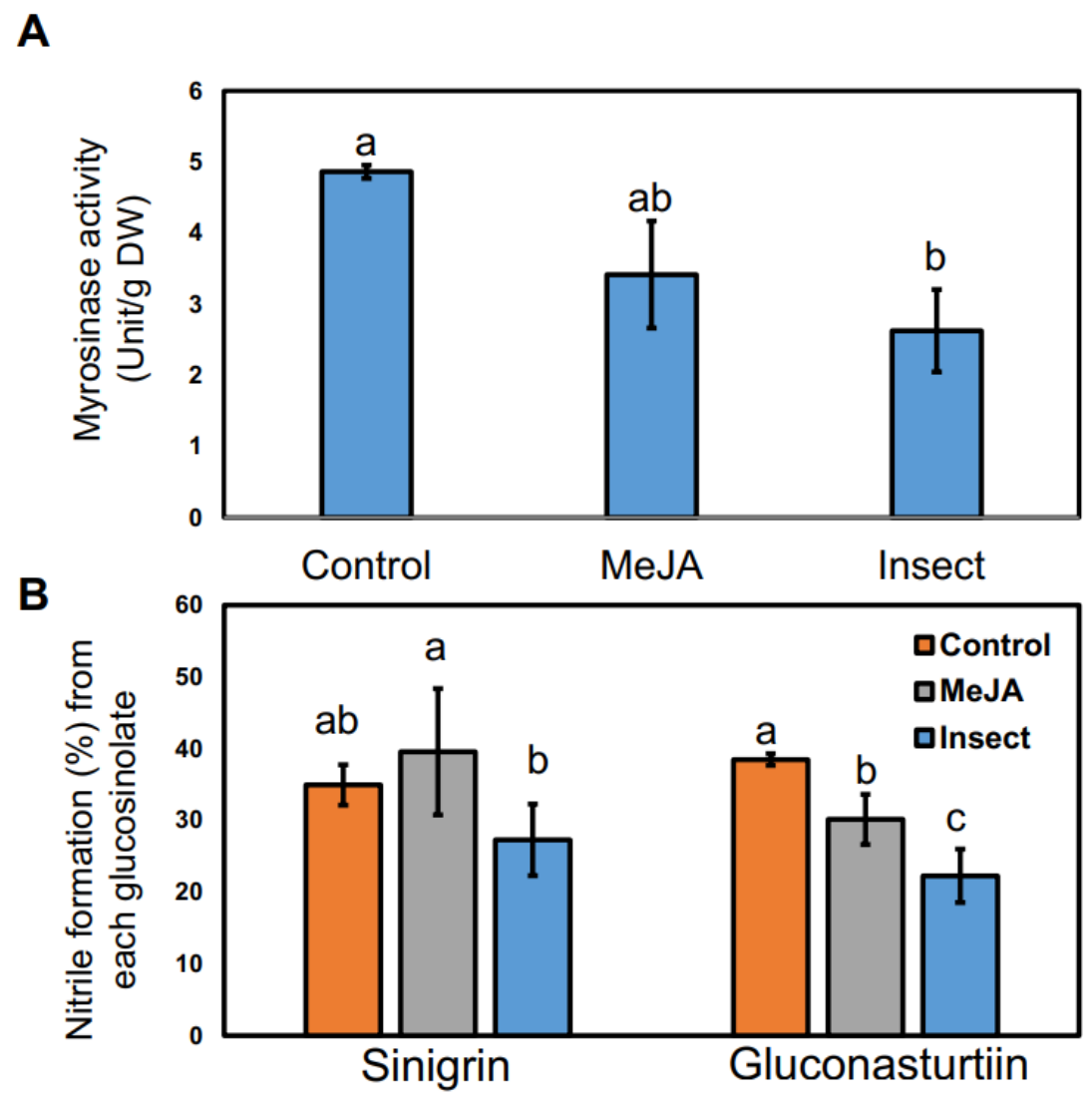

Figure 2.2 (A) Myrosinase activity (Unit/g DW) and (B) Nitrile formation (\%) of control, insectdamaged, and MeJA-treated kale.

Nitrile formation (\%) is shown as the relative ratio of nitrile to the total concentration of the hydrolysis products formed (sum of isothiocyanates and nitriles) from sinigrin and gluconasturtiin. The data are presented as the mean concentration \pm standard error $(n=3)$. Different letters mean significantly different by Student's $t$-test $(p<0.05)$ across three groups on (A) and three groups within precursor GS on (B), respectively. 
A

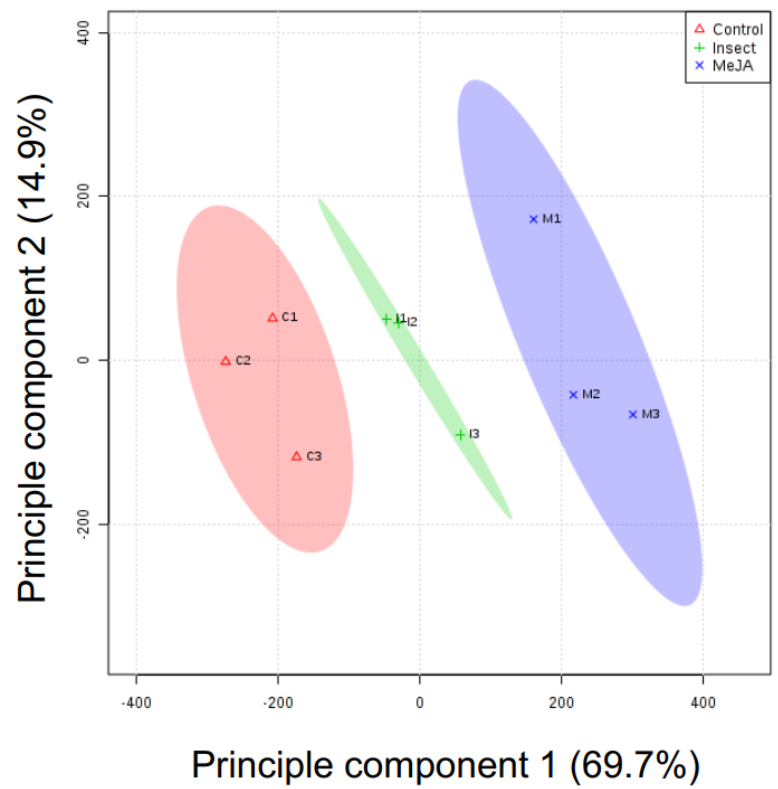

B

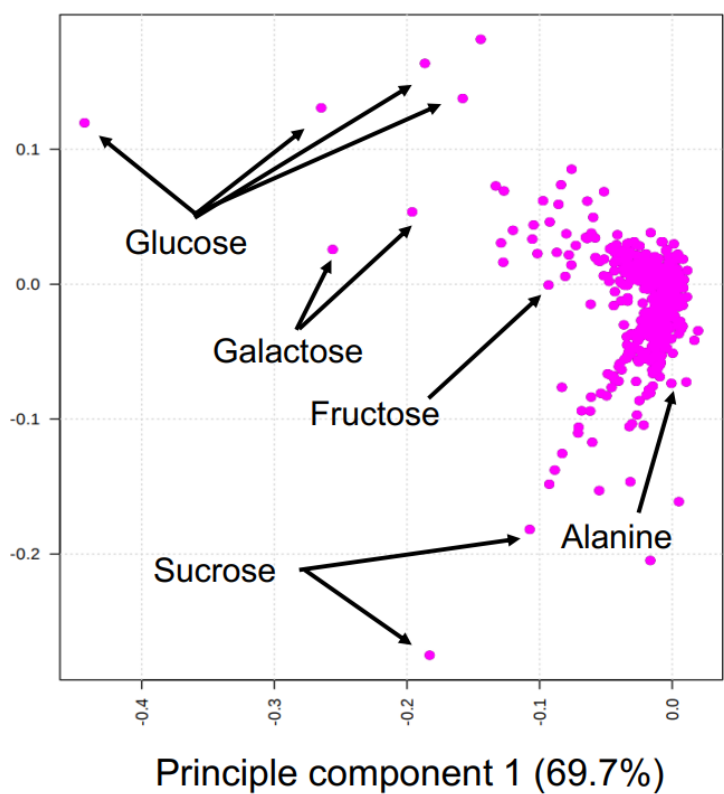

Figure 2.3 Principle component analysis (A) score and (B) loading plots derived from nontargeted metabolite profiling of Brassica napus with the control plants, MeJA-treated plants, and insect-feeding plants.

The shaded areas in (A) represents $95 \%$ confidence regions. The pink dots indicated by arrows in (B) represent discriminating biomarkers among various treatments. 

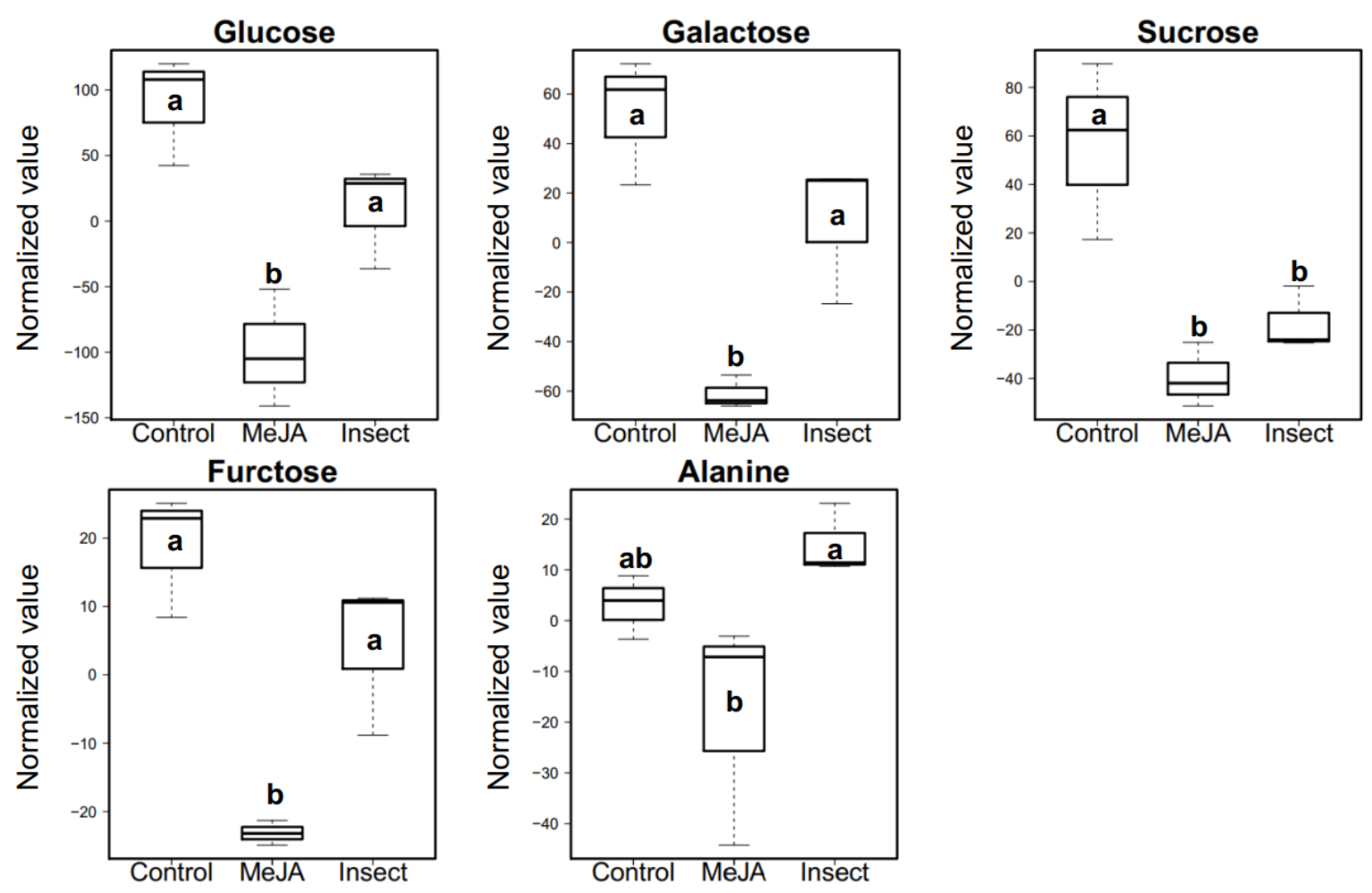

Figure 2.4 Five biomarker metabolites selected with top-5 variable importance in projection (VIP) scores (VIP > 1.2) from all metabolites among control kales, MeJA-treated kales, and insect-infected kales.

Different letter indicates significant differences between the groups, determined by Student's significance test $(p<0.05)$. The presented values are normalized values based on an internal standard with auto-scale in Metaboanalyst. 


\section{Chapter 3 Effect of methyl jasmonate treatment on glucosinolate retention and hydrolysis products after three cooking treatments ${ }^{2}$}

\subsection{Abstract}

Exogenous methyl jasmonate (MeJA) treatment will increase the levels of neoglucobrassicin and their bioactive hydrolysis products in broccoli (Brassica oleracea var. italica), although the fate of MeJA-induced glucosinolates (GS) after various cooking methods is unknown. This study measured the changes in GS and their hydrolysis compounds in broccoli treated with MeJA and the interaction between MeJA and cooking treatments. All cooked MeJA-treated broccoli contained significantly more GS than untreated broccoli $(\mathrm{P}<0.05)$. After 5 min of cooking (boil, steam, microwave), MeJA-treated broccoli contained 1.6-2.3-fold higher GS than the untreated broccoli. The retention rate of total GS in steamed and microwaved MeJA-treated broccoli was significantly higher $(\mathrm{P}<0.05)$. Neoglucobrassicin hydrolysis products were significantly greater in steamed and microwaved MeJA-treated broccoli than that in untreated broccoli. The results show that exogenous MeJA treatment increases neoglucobrassicin and their hydrolysis compounds in broccoli even after cooking which may enhance the nutritional quality.

Keywords: Broccoli, methyl jasmonate, glucosinolate, glucosinolate hydrolysis products, cooking

\footnotetext{
${ }^{2}$ This chapter is submitted to Journal of Food Science and it is currently under review.
} 


\section{Practical Application:}

Exogenous methyl jasmonate (MeJA) treatment in broccoli will increase the levels of glucosinolates (GS), a group of phytochemicals associated with anti-cancer and liver health benefits. The results showed that exogenous MeJA treatment will significantly increase GS and their hydrolysis compounds in broccoli. These compounds will be retained more in MeJA-treated broccoli even after cooking. MeJA-treated broccoli has potential for use as a value-added ingredient and since MeJA can be applied to other Brassica vegetables, this treatment may be used to enhance the nutritional value of other commodities. 


\subsection{Introduction}

The consumption of broccoli (Brassica oleracea var. italica) is associated with anticancer activity in in vitro and in vivo trials due to the high content of phytochemicals, minerals, vitamins, and fibers. Glucosinolates (GS) are a group of phytochemicals in broccoli which are studied extensively for their health benefits; for example, an inverse relationship has been reported between the risk of breast cancer in Caucasian women and consumption of broccoli (Ambrosone et al., 2004). Furthermore, the consumption of broccoli was shown to reduce the concentration of hepatic triglycerides in mice, and long-term broccoli consumption may promote liver health (Chen, Wallig, \& Jeffery, 2016).

GS can be categorized based on the precursor amino acids. Aliphatic GS are derived from methionine and indolyl GS are derived from tryptophan (Halkier \& Gershenzon, 2006). In plants, GS are hydrolyzed by an endogenous enzyme myrosinase (EC 3.2.3.1, thioglucoside glucohydrolase) when insects attack or a physical wound is formed. This reaction produces the corresponding hydrolysis products. For example, isothiocyanates (ITCs) are GS hydrolysis products reported to possess generally higher bioactivity than other types of GS hydrolysis products (Shapiro, Fahey, Wade, Stephenson, \& Talalay, 2001). The chemopreventive effect of sulforaphane, a well-known ITC, is greater than other GS hydrolysis products in cell culture assay (Ku et al., 2013), animal assay (Hu et al., 2006), and in a clinical trial (Ambrosone et al., 2004). However, the accumulation of its precursor, glucoraphanin, is mainly determined by the genetic background of the plant rather than the environmental factors (Brown et al., 2002); therefore, increasing its concentration through agricultural practices is limited. In contrast, indolyl GS levels are mainly affected by environmental factors (Brown et al., 2002), which implies that the concentration of indolyl GS can be increased by agricultural practices. 
Broccoli is often eaten cooked, or sometimes eaten raw as an ingredient in salads. Several studies have investigated the impact of common cooking methods including boiling, steaming, and microwaving on the retention of GS, with boiling generally causing substantial loss of GS (Jones, Frisina, Winkler, Imsic, \& Tomkins, 2010; Kapusta-Duch, Kusznierewicz, Leszczyńska, \& Borczak, 2016; Sarvan, Verkerk, van Boekel, \& Dekker, 2014; Vallejo, Tomás-Barberán, \& García-Viguera, 2002; Yuan, Sun, Yuan, \& Wang, 2009). Although other common cooking methods such as steaming and microwaving do not cause GS losses as substantial as boiling, both methods were reported to decrease aliphatic and indolyl GS (Palermo, Pellegrini, \& Fogliano, 2014). In addition to the GS loss, cooking also inactivates myrosinase and hinders the formation of hydrolysis products (Oliviero, Verkerk, \& Dekker, 2018). Therefore, although the loss of GS during domestic cooking has been extensively investigated in broccoli, methods of delivering nutritionally-enhanced broccoli after cooking are still lacking in the literature.

Exogenous methyl jasmonate (MeJA) application increases inducible indolyl GS (neoglucobrassicin and glucobrassicin) or aromatic GS (gluconasturiin) concentration in Brassica vegetables, which include broccoli (Ku, Jeffery, \& Juvik, 2014), due to its anti-repellent activity. MeJA is a volatile jasmonate derivative that responds to herbivore damage (Tamogami, Noge, Abe, Agrawal, \& Rakwal, 2012; Wu, Wang, \& Baldwin, 2008) and GS biosynthesis is upregulated by JA signaling transduction (Frerigmann \& Gigolashvili, 2014; Kim \& Juvik, 2011). Applying methyl jasmonate can mimic the defense response to insect damage (Thaler, Stout, Karban, \& Duffey, 1996); as a result, defense-related metabolites such as GS, are upregulated (Kim \& Juvik, 2011) either by depletion of the carbon pool (glucose) or by the conserved transcriptional network (Campos et al., 2016). MeJA application can be used as an agricultural practice to enhance the nutritional quality of broccoli. Indole-3-carbinol (I3C; 
derived from glucobrassicin) was associated with reducing the risk of hormone-responsive cancers (Becker \& Juvik, 2016) including breast (Jump et al., 2008) and prostate cancer (Sarkar \& Li, 2004). N-methoxy-indole-3-carbinol (NMI3C), derived from neoglucobrassicin, was reported to reduce the growth of human colon cells by inhibiting CYP1A enzyme, and its potency was higher than I3C in vitro (Neave, Sarup, Seidelin, Duus, \& Vang, 2005).

MeJA treatment will also change different aspects of the plant cell; for instance, it modifies cell wall composition of loquat fruit (Cao, Zheng, Wang, Rui, \& Tang, 2010) and increases the cellulose content of the model grass Brachypodium distachyon (Napoleão et al., 2017). Therefore, we hypothesized that MeJA-induced changes in broccoli may affect GS degradation during domestic cooking. The aim of this study was to evaluate how MeJA application to broccoli plants will affect GS concentration and retention rate in broccoli florets after boiling, steaming, and microwaving treatments.

\subsection{Materials and methods}

\subsubsection{Broccoli cultivation and sample preparation}

Broccoli cultivar 'Green Magic' was chosen for its consistent response to $250 \mu \mathrm{M} \mathrm{MeJA}$ application year to year (Ku, Jeffery, \& Juvik, 2013). Seed of 'Green Magic' broccoli were purchased from Johnny's Selected Seeds (Albion, ME, USA) and germinated in flats with Sunshine \#1 Mix (Sun Gro Horticulture, Vancouver, BC, Canada) in the greenhouse facility at West Virginia University (WVU) for four weeks under a $25 / 18^{\circ} \mathrm{C}$ and $14 / 10 \mathrm{~h}$ day/night regime with supplemental high-pressure sodium lighting (600 W HS200 deep reflector; Hortilux, Pijnacker, Netherlands) when the light intensity was below $50 \mathrm{~W} / \mathrm{m}^{2}$. The seedlings were 
transplanted to $15-\mathrm{cm}$ pots for another three weeks and then transplanted to $8.7-\mathrm{L}$ pots. Forty untreated broccoli heads were harvested eight weeks after transplanting. Another 40 plants were subjected to treatment with $250 \mu \mathrm{M}$ MeJA in $0.1 \%$ Triton X-100 (Sigma-Aldrich, St. Louis, MO, USA) solution three days before harvesting. The harvested broccoli heads were stored at $4{ }^{\circ} \mathrm{C}$ prior to cooking.

\subsubsection{Broccoli preparation and cooking process}

The harvested broccoli heads were chopped into 3-4-cm wide florets. After chopping, broccoli branchlets were randomized and weighed $100 \mathrm{~g}$ as one replicate for each cooking method. Three replicates were used to represent the result from each treatment. Broccoli was cooked for 2 min and 5 min using each cooking method to evaluate metabolite loss as a function of cooking time. For all three cooking methods, 2-min treatment and 5-min treatment was conducted separately so no interruption occurred in 5-min treatment.

Seven hundred milliliters of water were used for both boiling and steaming treatments. For boiling treatment, the broccoli florets were immersed in boiling water for 2 or 5 min, following which, they were immediately removed from the boiling water and transferred to an ice water bath. For steaming treatment, the broccoli florets were evenly spread out without any overlap on a stainless-steel steamer basket, which was placed over boiling water for 2 and 5 mins. Steamed broccoli was immediately transferred to an ice water bath for rapid cooling. For microwaving, a microwave oven (Whirlpool Corporation, Benton Harbor, MI, 49022) was used at 50\% power (maximum power: $1000 \mathrm{~W}$ ). The broccoli florets were placed on a plate and covered with a paper towel soaked in $50 \mathrm{~mL}$ water. The broccoli samples were microwaved for 2 or 5 min and were then transferred to an ice water bath for cooling. All cooked broccoli samples were packed 
in a zipper bag, which were then placed in a 68-L container (Sterilite, Townsend, MA, 01469) and moved to a $-20^{\circ} \mathrm{C}$ walk-in cooler prior to lyophilization.

The volume of the cooking water of the boiling and steaming treatments was adjusted to 700 $\mathrm{mL}$ before collecting in $50 \mathrm{~mL}$ twist tubes. For the microwaved sample, the damp paper towel was first rinsed in $200 \mathrm{~mL}$ water, after which the water was manually squeezed out of the paper towel and the volume was adjusted to $700 \mathrm{~mL}$. All cooking water samples were stored in a $4{ }^{\circ} \mathrm{C}$ walk-in cooler for further analysis.

\subsubsection{Quantification of glucosinolates}

GS in samples were analyzed using a previous published method (Ku \& Juvik, 2013) with slight modifications. Freeze-dried powdered material was extracted with $70 \%$ methanol at $95^{\circ} \mathrm{C}$ for $10 \mathrm{~min}$. Subsequently, the extract was analyzed on DEAE Sephadex A-25 (GE Healthcare, Piscataway, NJ, USA). The proteins in the extract were precipitated with $0.15 \mathrm{~mL}$ of a 1:1 mixture of $1 \mathrm{M}$ lead acetate and $1 \mathrm{M}$ barium acetate and centrifuged at 12,000 $\times g$ for 3 min. Each sample was loaded onto a column containing $1 \mathrm{M} \mathrm{NaOH}$ and $1 \mathrm{M}$ pyridine acetatecharged DEAE Sephadex A-25 resin (GE Healthcare, Piscataway, NJ, USA) for desulfation by Helix pomatia type-1 arylsulfatase (Sigma-Aldrich, St. Louis, MO, USA) and incubated for desulfation of GS overnight. Desulfo-GS was eluted with deionized distilled water, followed by filtration through a $0.2-\mu \mathrm{m}$ Nylon filter. The filtered sample was injected into a Nexera-i LC 2040C ultrahigh performance liquid chromatography (UHPLC) (Shimadzu, Kyoto, Japan) system equipped with photo diode array detector. A $100 \mathrm{~mm} \times 2.1 \mathrm{~mm}$ i.d., $1.8 \mu \mathrm{m}, 100 \AA$, Kromasil RP-C18 column was used (AkzoNobel, Bohus, Sweden). The detection of GS was at $229 \mathrm{~nm}$. The flow rate was $1 \mathrm{~mL} / \mathrm{min}$ with mobile phase A (deionized distilled water) and B (100\% acetonitrile) with the following elution profile: $0 \min 0 \%$ B, 7 min 4\% B, 20 min 20\% B, 
$35 \min 25 \%$ B, $36 \min 80 \%$ B, 40 min $80 \%$ B, $41 \min 0 \%$ B, and 50 min 0\% B. Glucosinalbin was used as an internal standard because it is not present in broccoli. For quantification, relative response factor (RRF) of glucosinalbin was set as 1 (Clarke, 2010). The UV response factors for various glucosinolates (Kusznierewicz, Iori, Piekarska, Namieśnik, \& Bartoszek, 2013) applied for quantification were: glucoiberin 1.07, progoitrin 1.09, glucoraphanin 1.07, sinigrin 1.00, gluconapin 1.11, glucoerucin 1.00, glucobrassicin 0.29 , 4-hydroxyglucobrassicin 0.28 , 4methoxyglucobrassicin 0.25 , gluconasturtiin 0.95 , and neoglucobrassicin 0.20 . RRF of 1hydroxyglucobrassicin was set as 0.28 (similar structure to 4-hydroxyglucobrassicin) because RRF was not available.

GS in cooking water was determined using the same protocol mentioned above with slight modification. Protein in GS cooking water was precipitated with $0.37 \mathrm{~mL}$ of a 1:1 mixture of 1 $\mathrm{M}$ lead acetate and $1 \mathrm{M}$ barium acetate and followed the same steps described in the last section.

\subsubsection{Quantification of GS hydrolysis products}

Freeze-dried powdered material $(50 \mathrm{mg})$ was suspended in $1 \mathrm{~mL}$ distilled water in a $2 \mathrm{~mL}$ Teflon tube (Fisher Scientific, Waltham, MA, USA). Hydrolysis products were generated naturally by the endogenous myrosinase in the absence of light at room temperature for $24 \mathrm{~h}$. After adding $1 \mathrm{~mL}$ dichloromethane, the samples were vigorously mixed and centrifuged at $12,000 \times g$ for $2 \mathrm{~min}$; the lower organic layer was collected and analyzed using gas chromatography (Trace 1310 GC, Thermo Fisher Scientific, Waltham, MA, USA) coupled to a mass spectrum (MS) detector system (ISQ QD, Thermo Fisher Scientific, Waltham, MA, USA) and an autosampler (Triplus RSH, Thermo Fisher Scientific, Waltham, MA, USA). A capillary column (Rxi-5Sil MS, Restek, Bellefonte, PA, USA; 30 m, 0.25 mm, 0.25 m, capillary column w/10 m Integra-Guard Column) was used. After an initial temperature hold at $40^{\circ} \mathrm{C}$ for $2 \mathrm{~min}$, 
the oven temperature was increased to $320^{\circ} \mathrm{C}$ at $15^{\circ} \mathrm{C} \min ^{-1}$ and held for $4 \mathrm{~min}$. The injector and detector temperatures were set at $270^{\circ} \mathrm{C}$ and $275^{\circ} \mathrm{C}$, respectively. The flow rate of the helium carrier gas was set at $1.2 \mathrm{~mL} \mathrm{~min}^{-1}$. The peaks were identified using information from a previous publication (Kim et al., 2017) or after comparison with the data in the National Institute of Standards and Technology (NIST) library.

\subsubsection{Quantification of myrosinase activity and nitrile formation}

Myrosinase activity and the percentage of nitrile formed from GS among the total hydrolysis products were measured to estimate epithiospecifier protein (ESP) levels and the epithiospecifier modifier 1 (ESM1) interaction based on published methods (Kim, Chiu, \& Ku, 2017).

Myrosinase activity was assessed as the total hydrolysis products generated in $60 \mathrm{~min}$. One unit $=1 \mu \mathrm{mol}$ total hydrolysis products released per minute. Nitrile formation was determined by incubating concentrated horseradish root extract with protein extracts from broccoli samples. Horseradish extract was used because it served as an exogenous substrate source of sinigrin and gluconasturtiin at a saturated level which minimizes the reaction with endogenous glucosinolate substrates from the different broccoli samples (Ku, Jeffery, Juvik, \& Kushad, 2015). Freezedried powdered samples $(75 \mathrm{mg})$ were mixed with $1.5 \mathrm{~mL}$ concentrated horseradish root extract in $2 \mathrm{~mL}$ microcentrifuge tubes. After centrifugation at $12,000 \times g$ for $2 \mathrm{~min}, 0.5 \mathrm{~mL}$ supernatant samples were transferred to $1.5 \mathrm{~mL}$ Teflon centrifuge tubes (Savillex Corporation, Eden Prairie, MN, USA) and $0.5 \mathrm{~mL}$ of dichloromethane was added. The tubes were placed upside down to minimize loss of volatile compounds at room temperature for $10 \mathrm{~min}$. Tubers were then vortexed and centrifuged at $12,000 \times g$ for $4 \mathrm{~min}$. The dichloromethane organic layer was injected into the GC-MS system described above to determine the GS hydrolysis products. The condition of temperature ramp was the same as in section 2.4. Standard curves of allyl isothiocyanate, 2- 
phenthyl isothiocyanate, and 3- phenylpropionitrile (Sigma-Aldrich, St Louis, MO, USA) were used for quantification. The standard curve of allyl isothiocyanate was also applied to quantify 1cyano-2,3-epithiopropane.

\subsubsection{Measurement of Electrical Conductivity in Cooking Water}

Electrical conductivity (EC) was measured using TechPro II ${ }^{\mathrm{TM}}$ (Myron L®, Carlsbad, CA, USA). The probe was calibrated first with deionized water. Deionized water was also used to rinse out the sampling probe thrice between measurements and the sampling probe was dried using Kim wipes before the measurement. EC was measured thrice for each cooking water sample and the averaged value of each sample was used for statistical analysis.

\subsubsection{Untargeted primary metabolites by GC-MS}

Primary metabolites were extracted using a published protocol (Lisec, Schauer, Kopka, Willmitzer, \& Fernie, 2006) with modifications. The samples (50 mg) were weighed in $2 \mathrm{~mL}$ microcentrifuge tubes, followed by the addition of ribitol as an internal standard, and extraction with $1.4 \mathrm{~mL}$ methanol at $75^{\circ} \mathrm{C}$. After cooling, the sample extracts were centrifuged, and the supernatants were transferred to new $2 \mathrm{~mL}$ microcentrifuge tubes. To fractionate polar compounds, $0.375 \mathrm{~mL}$ cold chloroform $\left(-20^{\circ} \mathrm{C}\right)$ and $0.7 \mathrm{~mL}$ cold water $\left(4^{\circ} \mathrm{C}\right)$ were added. After vigorous mixing, the extracts were centrifuged and transferred to $1.5 \mathrm{~mL}$ tubes. The extracts were dried using a Vacufuge ${ }^{\mathrm{TM}}$ concentrator (Eppendorf, Thermo Fisher Scientific, Waltham, MA, USA) and the dried extracts were derivatized with methoxyamine hydrochloride for 90 min at $37^{\circ} \mathrm{C}$, followed by $70 \mu \mathrm{L} \mathrm{N}$-Methyl- $N$-(trimethylsilyl) trifluoroacetamide (MSTFA) with $1 \%$ trimethylchlorosilane (TMCS) at $37^{\circ} \mathrm{C}$ for $30 \mathrm{~min}$. The metabolites were analyzed using the same GC-MS in section 2.4. The mass spectrometer was operated in positive electron impact mode (EI) at $70.0 \mathrm{eV}$ ionization energy at $\mathrm{m} / \mathrm{z} 40-500$ scan range. 
The acquired chromatograms were converted to mzXML using the RawConverter (He, Diedrich, Chu, \& Yates, 2015). Peak detection and alignment were performed by XCMS package in $\mathrm{R}$ language with default settings (Xia \& Wishart, 2002). All data was normalized to unique ion peak $(319 \mathrm{~m} / \mathrm{z}$ ) from the internal standard in the online platform MetaboAnalyst (Xia \& Wishart, 2002), and further statistical analyses were conducted after Pareto scaling. Metabolite identification was based on standard compounds (STD) in comparison with the mass spectra present in NIST library and retention times.

\subsubsection{Univariate and multivariate analyses}

Statistical analyses were conducted using JMP 14 (SAS Institute, Cary, NC, USA). Twoway univariate analysis of variance (two-way ANOVA) was used to determine the effect of MeJA treatment and cooking method on the metabolites. Data was separated into (A) raw and 2minute cooking and (B) raw and 5-minute cooking when two-way ANOVA was used. Slice test was used to determine the effect of MeJA on metabolites when the interaction between two factors (MeJA treatment and cooking method) was significantly interacted at $P \leq 0.05$. If no interaction was detected, Student t-test was used to determine the effect of MeJA on metabolites under the same cooking method and the significance was at $P \leq 0.05$.

For primary metabolites analysis, the acquired chromatograms converted to mzXML were used to conduct partial lease square-discrimination analysis, variable importance in projection values estimate, and ANOVA in MetaboAnalyst (Chong et al., 2018).

\subsection{Results and discussion}

\subsubsection{Effect of MeJA and cooking method on glucosinolate profile}


A total of $10 \mathrm{GS}$ were detected in broccoli samples and the results of two-way ANOVA (Table S1) showed significant interaction $(P \leq 0.05)$ between MeJA treatment and cooking method in total indolyl GS and total GS irrespective of the cooking time. The concentration of total GS in MeJA-treated broccoli was significantly higher $(P \leq 0.001)$ in MeJA-treated broccoli among all cooking methods (Figure 1). The concentration of total GS in raw, steamed and microwaved MeJA-treated broccoli was the greatest across all samples by post-hoc Tukey HSD test at $P \leq 0.05$ with no significant difference between each other. Boiling led to the maximum loss of GS among all MeJA-treated broccoli, which corresponded well with previous studies (Jones et al., 2010; Kapusta-Duch et al., 2016). However, the concentration of glucoraphanin was significantly reduced $(P \leq 0.05)$, which was different from previous study (Ku et al., 2013) that the concentration of glucoraphanin did not differ between control and $500 \mu \mathrm{M}$ MeJA-treated broccoli (Ku et al., 2013). The decrease may occur during postharvest since glucoraphanin was rapidly reduced in MeJA-treated broccoli 10 days after the harvest when stored at $4^{\circ} \mathrm{C}(\mathrm{Ku}$ et al., 2013).

The effects of MeJA on the GS retention rate were cooking method-dependent or GS type-dependent (Figure 2). MeJA treatment significantly increased the retention rate for total aliphatic GS, reduced the retention rate for total GS, or did not significantly affect total indolyl GS content. In the steamed and microwaved samples, the retention rate of total aliphatic GS, total indolyl GS, and total GS tended to be higher in MeJA-treated broccoli than in untreated broccoli (Figure 2). Taken together (Figures $1 \& 2$ ), the results suggest that MeJA-treatment not only increased the total GS in broccoli, but also improved the GS retention rate after cooking, especially after steaming and microwaving. 
Reports showed that the change of GS during the cooking process can depend on GS structure (Hanschen, Kühn, Nickel, Rohn, \& Dekker, 2018), plant matrix (Sarvan, Verkerk, van Boekel, \& Dekker, 2014), or cellular environment (Hanschen et al., 2012). The use of larger volumes of cooking water (boiling) also leads to higher loss of GS (Hwang \& Kim, 2013). GS loss during the cooking process may be attributed to cell lysis and thermal degradation (Hanschen et al., 2018). GS concentration in the cooking water was measured and varied between cooking methods. The greatest concentration of total GS in cooking water was detected in 5-min boiling water from control broccoli (Table 1). Results were consistent with previous literature where boiling was reported to lead to the great loss of GS (Hanschen et al., 2018; Song $\&$ Thornalley, 2007). Electrical conductivity (EC) of the cooking water was also measured (Table S3; Figure S1) but interaction from the two factors (MeJA treatment and cooking methods) were not detected ( $P=0.48$ for 2 -minute cooking broccoli and $P=0.12$ for 5 -minute cooking broccoli). The EC value of the boiling water of MeJA-treated and untreated broccoli was the highest among all the samples, which was indicative of high cell lysis (Jones et al., 2010).

MeJA treatment might change the cellular environment (Cheong \& Choi, 2003) and alter cell structure by changing the cell wall composition (Boonyaritthongchai, Chimvaree, Buanong, Uthairatanakij, \& Jitareerat, 2016; Cao et al., 2010; Napoleão et al., 2017), which affects leaching of GS from the cell and the differences in retention rate. In this study, total GS content in MeJA-treated broccoli was 1.6-fold higher than in untreated control broccoli after 5-min boiling, which was the most disruptive cooking method in this study (Figure 1). Notably, GS concentrations in MeJA-treated broccoli were significantly higher in all steamed samples and in the 5-min microwaving groups of total GS compared to the untreated broccoli. For milder 
cooking methods (2-min steaming or 2-min microwaving), the concentration of total GS in MeJA-treated broccoli was 2.5-fold higher than that in the untreated control broccoli. Many studies suggested that intact GS in food can be hydrolyzed via the myrosinase-like enzyme produced by the human gut microbiota (Tian, Liu, Lei, Zhang, \& Shan, 2018) while the efficiency may depend on individual and the type of microbes; therefore, MeJA-treated broccoli may deliver significantly more GS from broccoli in the human digestive system.

\subsubsection{Effect of MeJA treatment and cooking method on myrosinase activity and GS hydrolysis products in 'Green Magic' broccoli}

In raw broccoli, $250 \mu \mathrm{M}$ MeJA treatment significantly increased myrosinase activity by 37\% (Figure 3). We also indirectly measured epithiospecifier protein (ESP) activity by incubating broccoli crude protein and horseradish GS extract together (Ku et al., 2015). Nitrile formation (\%) of gluconaturiin was reduced, although the result was not statistically significant $(P=0.06)$.

A total of 11 GS hydrolysis products were detected in raw broccoli, including ITC, nitriles, indoles and oxazolidine-thione (Table S4). With the substantial increase in neoglucobrassicin and glucobrassicin after MeJA treatment (Table S2), the levels of the hydrolysis products derived from neoglucobrassicin (NMI3C, N-methoxyindole-3carboxyaldehyde (NMI3CA), $N$-methoxyindole-3-acetonitrile (NMI3ACN), and indole-3carbinol (I3C) from glucobrassicin were significantly higher $(P \leq 0.05)$ in raw MeJA-treated broccoli than in untreated broccoli. NMI3C and I3C were reported to have anti-inflammatory and chemopreventive effects (Fuentes, Paredes-Gonzalez, \& Kong, 2015; Neave et al., 2005); moreover, NMI3C may be a stronger inhibitor than I3C in tumor development (Stephensen et al., 2000). Therefore, the increase in the levels of these compounds after MeJA treatment might 
improve the nutritional value of raw broccoli. NMI3C and NMI3ACN levels were significantly higher in MeJA-treated broccoli for most of the cooking methods (Table 2). However, other GS hydrolysis products were not affected by the MeJA treatment (Table S5); therefore, the effect of MeJA on hydrolysis products may vary with precursor GSs.

Myrosinase activity was close to zero in all the cooked samples (Figure 3). Myrosinase inactivation during cooking process may reduce the content of all hydrolysis products as no significant difference of hydrolysis product levels were observed between the majority of cooked MeJA-treated and untreated broccoli. Therefore, although MeJA treatment might increase myrosinase activity in raw material, the effect was not observed after 2-min boiling, steaming, or microwaving (Hanschen et al., 2018).

\subsubsection{Effect of MeJA and cooking method on primary metabolites in 'Green Magic' broccoli with or without MeJA treatment}

Studies have shown that MeJA may change primary metabolites including sugar, amino acid, and organic acid by regulating the balance between growth and defense activities (Havko et al., 2016; Major et al., 2017). These primary metabolites can be valuable nutrition components; however, the effect of MeJA treatment on primary metabolites in cooked broccoli has not been studied. Partial least square-discrimination analysis (PLS-DA) was used to identify potential biomarkers with a variable importance in projection (VIP) value over 1.5, which indicates that this metabolite contributed greatly to the differences between groups (Table S5).

In the raw broccoli, MeJA treatment significantly reduced $(P \leq 0.05)$ oxoproline, glutamic acid, myo-inositol, and sucrose content (Table 3). This was consistent with published studies where MeJA treatment reduced sugars and amino acids in other Brassica vegetables 
(Chiu, Juvik, \& Ku, 2018; Kim, et al., 2017). Therefore, MeJA-mediated reduction in sugar and amino acid content was common for many Brassica species. The VIP values of primary metabolites, including sucrose, glucose, fructose, myo-inositol, oxoproline, and quininic acid, were consistently high (>1.5) with all cooking methods; hence, changes in the levels of these compounds were due to the effect of MeJA and not because of cooking methods. In other words, $250 \mu \mathrm{M}$ MeJA treatment changed the amount of individual primary metabolites in raw broccoli, and this effect was still observed after all cooking methods. MeJA treatment significantly increased the amount of quinic acid in the 2-min boiling, 2 and 5-min steaming, and 2 and 5-min microwaving samples. The levels of amino acids (oxoproline, glutamic acid, valine, isoleucine, proline, serine, and alanine) were significantly reduced by MeJA treatment, and those of glutamic acid, proline, and serine were reduced by $>50 \%$ (Table 2). The amount of sucrose and myo-inositol were significantly lower in all MeJA-treated cooked broccoli; however, the amount of glucose and fructose showed opposite trends. MeJA-treated broccoli contained significantly higher amounts of glucose after 2 min boiling and microwaving. In addition, the amount of fructose was significantly higher after 5 min microwaving of MeJA-treated broccoli.

It has been reported that amino acids can taste sweet, sour, bitter, or umami (Nishimura \& Kato, 1988). For example, glutamate is sometimes used as an umami ingredient in culinary practices. In this study, amino acids associated with bitterness (valine and isoleucine), sweetness (alanine, proline, and serine), and umami (glutamic acid) were all significantly changed by 250 $\mu \mathrm{M}$ MeJA treatment. Sugars, such as sucrose, are associated with masking the bitterness of Brassica vegetables (Beck, Jensen, Bjoern, \& Kidmose, 2014), and glucose correlates highly with the perception of sweetness (Chadwick, Gawthrop, Michelmore, Wagstaff, \& Methven, 2016). In fact, $250 \mu \mathrm{M}$ MeJA treatment on 'Green magic' broccoli was found to change the 
sensorial perception of raw broccoli but not of cooked broccoli, as determined by a consumer panel. The perceptible changes were mainly attributed to neoglucobrassicin and its hydrolysis products, not amino acids or sugars (Chiu, Matak, \& Ku, 2019).

Collectively, MeJA-treated broccoli tended to contain less sugars and amino acids even after domestic cooking methods, which may affect the nutritional value of the broccoli since amino acids are involved in various biochemical processes and immunity of the human body (Sikalidis, 2015; Wu, 2010). Understanding the changes of primary metabolites in response to MeJA treatment and the mechanism via which they change during cooking may provide valuable insights regarding the nutritional value of broccoli.

\subsection{Conclusion}

In this study, broccoli treated with $250 \mu \mathrm{M}$ MeJA contained significantly greater concentrations of GS than untreated broccoli even after cooking by boiling, steaming and microwaving. Exogenous $250 \mu \mathrm{M}$ MeJA application will increase the nutritional value of cooked broccoli because of the increased concentration of GS and retention of these compounds after cooking. MeJA-treated broccoli has potential for use as a value-added ingredient and since MeJA can be applied to other Brassica vegetables, this treatment may be used to enhance the nutritional value of other commodities.

\section{Author contributions}

$\mathrm{Ku}$ and Chiu designed the study and conducted the experiments. Chiu took the lead in analyzing data and writing the manuscript in consultation with Ku and Matak. All authors helped shape the research, analysis, and manuscript. 
Table 3.1 Glucosinolate profile ( $\mu$ mole $\cdot \mathrm{g}^{-1} \mathrm{DW}$ ) in the cooking water collected from (A)2-minute cooked broccoli and (B) 5-minute cooked broccoli with or without $250 \mu \mathrm{M}$ methyl jasmonate application (average $\pm \mathrm{SD}, \mathrm{n}=3$ ).

\begin{tabular}{ccccc}
\hline \multirow{2}{*}{ Boiling } & \multicolumn{2}{c}{ 2-min cooking } & \multicolumn{2}{c}{$\begin{array}{c}\text { 5-min cooking } \\
\text { Total } \boldsymbol{G S}\end{array}$} \\
\hline \multirow{3}{*}{ Steaming } & Control & $0.206 \pm 0.008$ & Control & $0.259 \pm 0.019$ \\
& MeJA & $0.467 \pm 0.607^{\text {ns }}$ & MeJA & $0.109 \pm 0.002^{* * *}$ \\
& Control & $0.017 \pm 0.006$ & Control & $0.024 \pm 0.010$ \\
\multirow{2}{*}{ Microwaving } & MeJA & $0.024 \pm 0.002^{\text {ns }}$ & MeJA & $0.041 \pm 0.003^{*}$ \\
& Control & $0.068 \pm 0.022$ & Control & $0.051 \pm 0.022$ \\
& MeJA & $0.013 \pm 0.004^{*}$ & MeJA & $0.159 \pm 0.046^{*}$ \\
\hline
\end{tabular}

Asterisk (*) indicates significant difference with or without MeJA within the same cooking treatment by Student's T-test $(P \leq 0.05, N=3)$.

ns , not significant; *, $P \leq 0.05 ; * *, P \leq 0.01 ; * * *, P \leq 0.001$. 
Table 3.2 Peak intensity $\left(\times 10^{3}\right)$ of neoglucobrassicin-derived hydrolysis products and total glucosinolate hydrolysis products of raw and cooked broccoli with or without $250 \mu \mathrm{M}$ methyl jasmonate application (average $\pm \mathrm{SD}, \mathrm{n}=3$ ).

Compound identification were based on a previous publication (Kim et al., 2017) or by comparison with data in the National Institute of Standards and Technology (NIST) library.

\begin{tabular}{clllll}
\hline $\begin{array}{c}\text { Cooking } \\
\text { method }\end{array}$ & Treatment & NMI3CA $^{\dagger}$ & NMI3ACN ${ }^{*}$ & NMI3C & Total Peak Intensity \\
\hline Raw & Control & $35.00 \pm 11.30$ & $0.34 \pm 0.33$ & $450.5 \pm 39.6$ & $494.4 \pm 50.1$ \\
& MeJA & $116.13 \pm 38.96^{*}$ & $0.65 \pm 0.17^{\mathrm{ns}}$ & $3991.3 \pm 539.4^{* * *}$ & $4119.1 \pm 580.0^{* * *}$ \\
\hline 2-minute & Control & $5.89 \pm 0.76$ & $0.29 \pm 0.22$ & $66.8 \pm 9.0$ & $83.13 \pm 61.65$ \\
Boiling & MeJA & $13.70 \pm 3.36^{*}$ & $0.69 \pm 0.30^{\mathrm{ns}}$ & $53.1 \pm 48.0^{\mathrm{ns}}$ & $77.17 \pm 47.75^{\mathrm{ns}}$ \\
\hline 5-minute & Control & $4.00 \pm 0.62$ & $0.49 \pm 0.18$ & $399.83 \pm 345.51$ & $414.33 \pm 345.15$ \\
Boiling & MeJA & $15.69 \pm 4.73^{*}$ & $0.25 \pm 0.42^{\mathrm{ns}}$ & $812.7 \pm 371.8^{\mathrm{ns}}$ & $841.99 \pm 371.35^{\mathrm{ns}}$ \\
\hline 2-minute & Control & $22.75 \pm 5.79$ & $0.49 \pm 0.14$ & $106.5 \pm 95.0$ & $161.66 \pm 82.70$ \\
Steaming & MeJA & $35.26 \pm 8.82^{\mathrm{ns}}$ & $0.44 \pm 0.12^{\mathrm{ns}}$ & $4.13 \pm 5.58^{\mathrm{ns}}$ & $50.92 \pm 12.06^{\mathrm{ns}}$ \\
\hline 5-minute & Control & $56.13 \pm 25.34$ & $0.60 \pm 0.58$ & $135.2 \pm 231.8$ & $213.59 \pm 234.45$ \\
Steaming & MeJA & $101.79 \pm 41.51^{\mathrm{ns}}$ & $1.23 \pm 1.23^{\mathrm{ns}}$ & $57.73 \pm 55.04^{\mathrm{ns}}$ & $172.19 \pm 78.93^{\mathrm{ns}}$ \\
\hline 2-minute & Control & $2.86 \pm 0.83$ & $0.49 \pm 0.10$ & $33.3 \pm 31.5$ & $49.28 \pm 36.33$ \\
Microwaving & MeJA & $7.25 \pm 0.38^{* *}$ & $0.15 \pm 0.18^{* *}$ & $523.5 \pm 67.1^{*}$ & $539.99 \pm 69.96^{* * *}$ \\
\hline 5-minute & Control & $28.35 \pm 10.68$ & $0.55 \pm 0.45$ & $180.02 \pm 114.76$ & $221.77 \pm 100.29$ \\
Microwaving & MeJA & $101.45 \pm 46.64^{\mathrm{ns}}$ & $0.46 \pm 0.42^{* *}$ & $2361.27 \pm 1006.49^{\mathrm{ns}}$ & $2472.72 \pm 1054.22^{*}$ \\
\hline
\end{tabular}

${ }^{\dagger} \mathrm{NMI} 3 \mathrm{CA}==N$-methoxyindole-3- carboxyaldehyde

NMI3ACN= N-methoxyindole-3-acetonitrile

${ }^{\S} \mathrm{NMI} 3 \mathrm{C}=N$-methoxyindole-3-carbinol

Asterisk $(*)$ indicate significant difference (Student t-test, $P \leq 0.05$ ) with or without MeJA treatment under the same cooking method.

ns , not significant; * $P \leq 0.05 ; * *, P \leq 0.01 ; * * *, P \leq 0.001$. 
Table 3.3 Fold changes of primary metabolites in MeJA-treated 'Green Magic' compared to untreated broccoli under the same cooking treatment.

\begin{tabular}{|c|c|c|c|c|c|c|c|c|c|c|c|c|c|}
\hline \multirow{2}{*}{\multicolumn{2}{|c|}{ Cooking method }} & \multicolumn{7}{|c|}{ Amino acids $\mathbf{s}^{\dagger}$} & \multicolumn{4}{|c|}{ Sugar and Sugar Derivatives } & \multirow{2}{*}{$\begin{array}{c}\text { Organic } \\
\text { Acids } \\
\text { Quinic } \\
\text { acid }\end{array}$} \\
\hline & & $\begin{array}{c}\text { Oxo- } \\
\text { proline }\end{array}$ & Glu & Val & Pro & Ser & Ile & Ala & Fructose & Glucose & Sucrose & $\begin{array}{c}\text { myo- } \\
\text { inositol }\end{array}$ & \\
\hline \multicolumn{2}{|c|}{ Raw } & $0.58 *$ & $0.42 *$ & & & & & & 0.94 & 1.06 & $0.28 *$ & $0.91^{*}$ & 1.35 \\
\hline \multirow[t]{2}{*}{ Boiling } & $2 \mathrm{~min}$ & $0.77 *$ & $0.35^{*}$ & & & & & & $0.96^{*}$ & $1.09 *$ & $0.26^{*}$ & $0.90 *$ & $1.35^{*}$ \\
\hline & $5 \mathrm{~min}$ & 0.95 & $0.43 *$ & & & & & & 0.80 & 1.09 & $0.37 *$ & 0.84 & 1.73 \\
\hline \multirow[t]{2}{*}{ Steaming } & $2 \mathrm{~min}$ & $0.71 *$ & $0.40^{*}$ & $0.49 *$ & $0.40 *$ & $0.39 *$ & $0.38 *$ & & & 1.05 & $0.32 *$ & $0.74 *$ & $1.72 *$ \\
\hline & $5 \mathrm{~min}$ & $0.70 *$ & $0.35 *$ & $0.43 *$ & $0.35 *$ & $0.40^{*}$ & $0.26^{*}$ & & & $0.90^{*}$ & $0.26^{*}$ & $0.59 *$ & $1.54 *$ \\
\hline \multirow{2}{*}{$\begin{array}{l}\text { Micro- } \\
\text { waving }\end{array}$} & $2 \min$ & $0.46^{*}$ & $0.43^{*}$ & 0.48 & $0.37^{*}$ & $0.36^{*}$ & & $0.69^{*}$ & 0.98 & $1.05^{*}$ & $0.26^{*}$ & $0.76^{*}$ & $2.04^{*}$ \\
\hline & $5 \mathrm{~min}$ & $0.62 *$ & $0.57 *$ & $0.57^{*}$ & $0.57^{*}$ & $0.56^{*}$ & & $0.86^{*}$ & $1.31^{*}$ & $1.38 *$ & $0.49 *$ & 1.02 & $2.33^{*}$ \\
\hline
\end{tabular}

$\dagger$ Abbreviation of amino acid: (1) Glu = glutamic acid, (2) Val = valine, (3) Pro = proline, (4) Ser = serine, (5) Ile = isoleucine, and (6) Ala $=$ alanine .

Asterisk (*) indicates a significant difference of the fold change compared to the untreated broccoli by Student's t-test at $P \leq 0.05$ based on peak intensity $(n=3)$ 


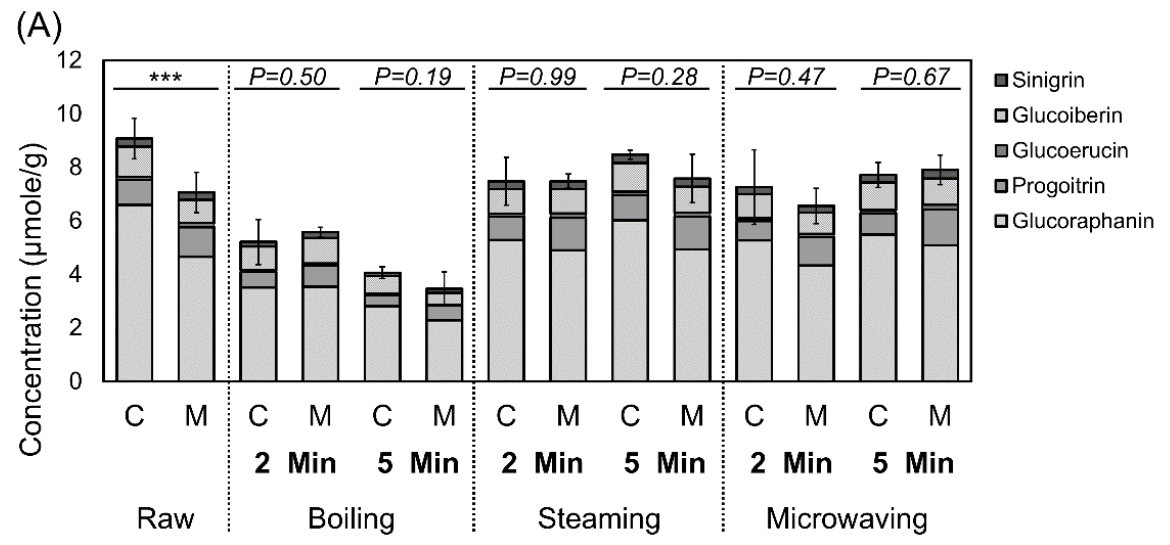

(B)

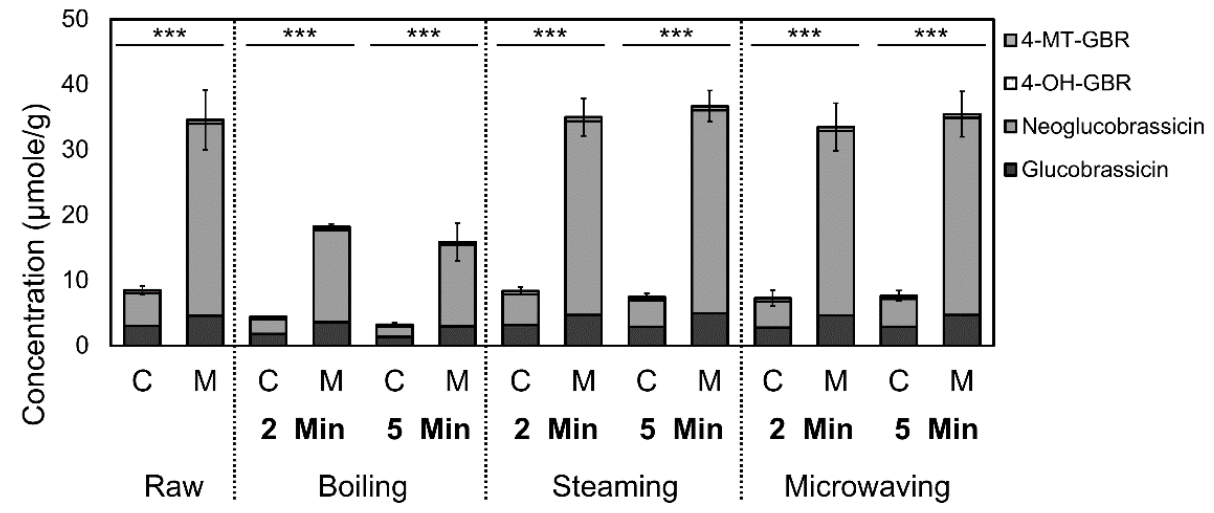

(C)

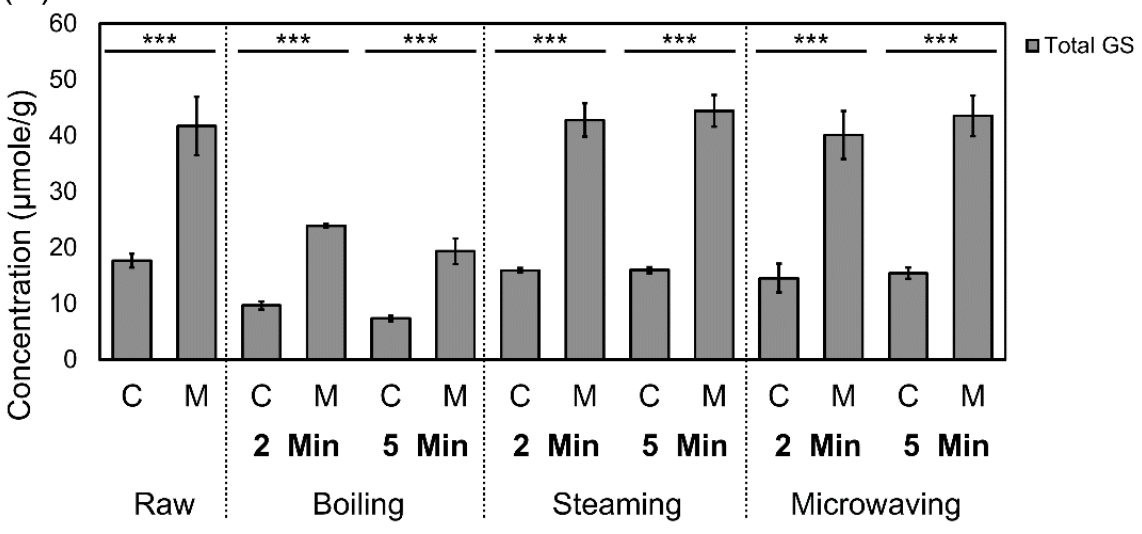

Figure 3.1. Effect of cooking and $250 \mu \mathrm{M}$ MeJA treatment on (A) total aliphatic glucosinolates, (B) total indolyl glucosinolates, and (C) total glucosinolates in 'Green Magic' broccoli.

C: control broccoli; M, MeJA-treated broccoli. Asterisk (*) indicates significant difference of (A) total aliphatic glucosinolates, (B) total indolyl glucosinolates, and (C) total glucosinolates with or without MeJA within the same cooking treatment by Student's T-test $(P \leq 0.05, N=3)$ with the significant interaction between MeJA treatment and cooking treatment detected (Supplementary Table S1).

*, $P \leq 0.05 ; * *, P \leq 0.01 ; * * *, P \leq 0.001$. 
(A)

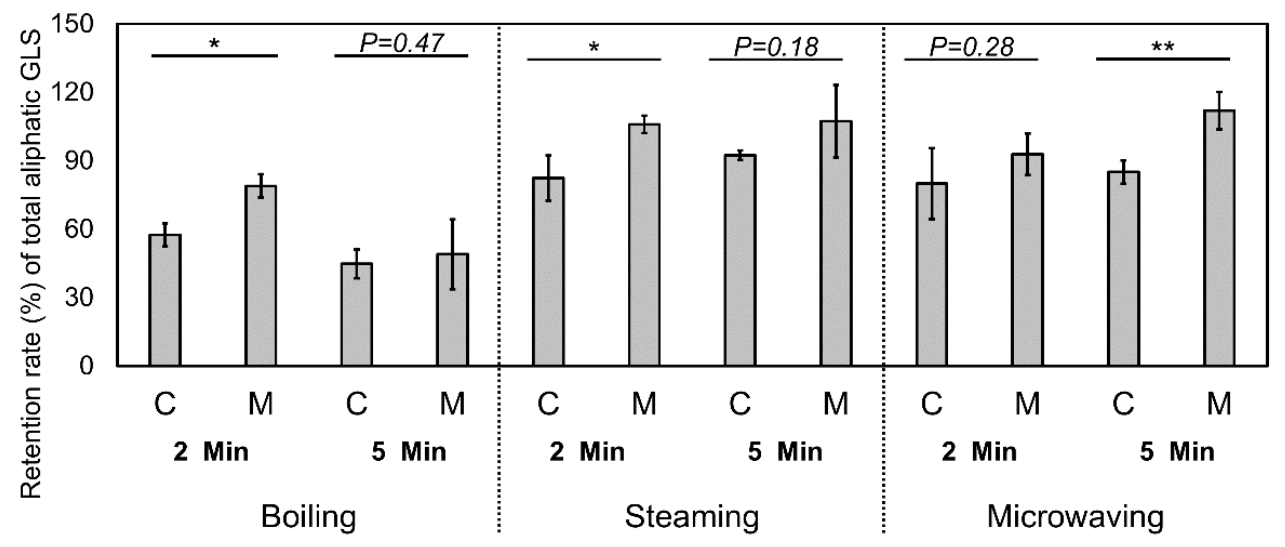

(B)

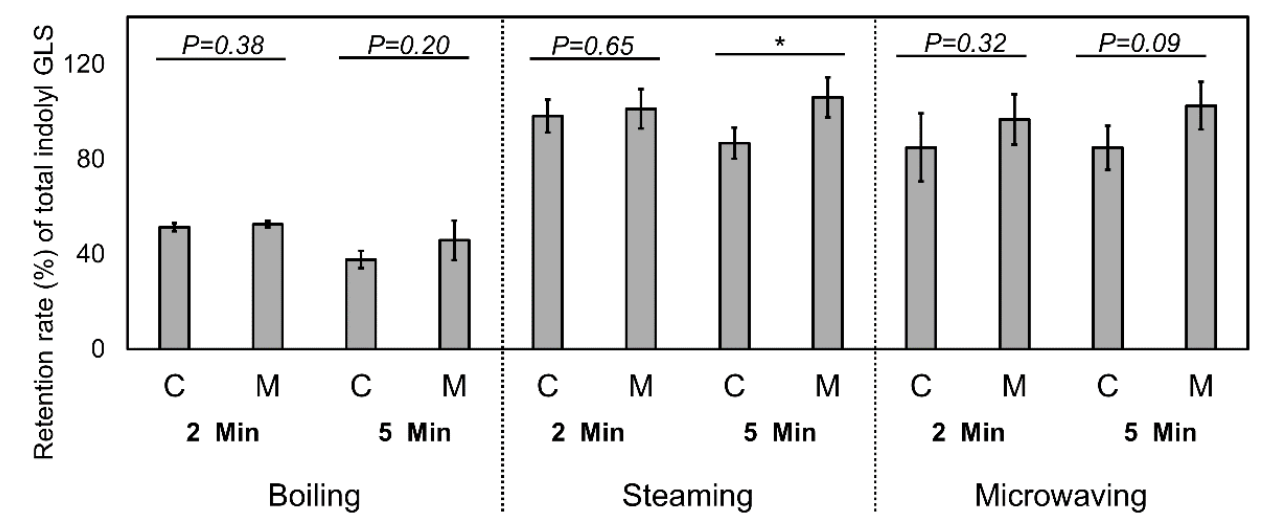

(C)

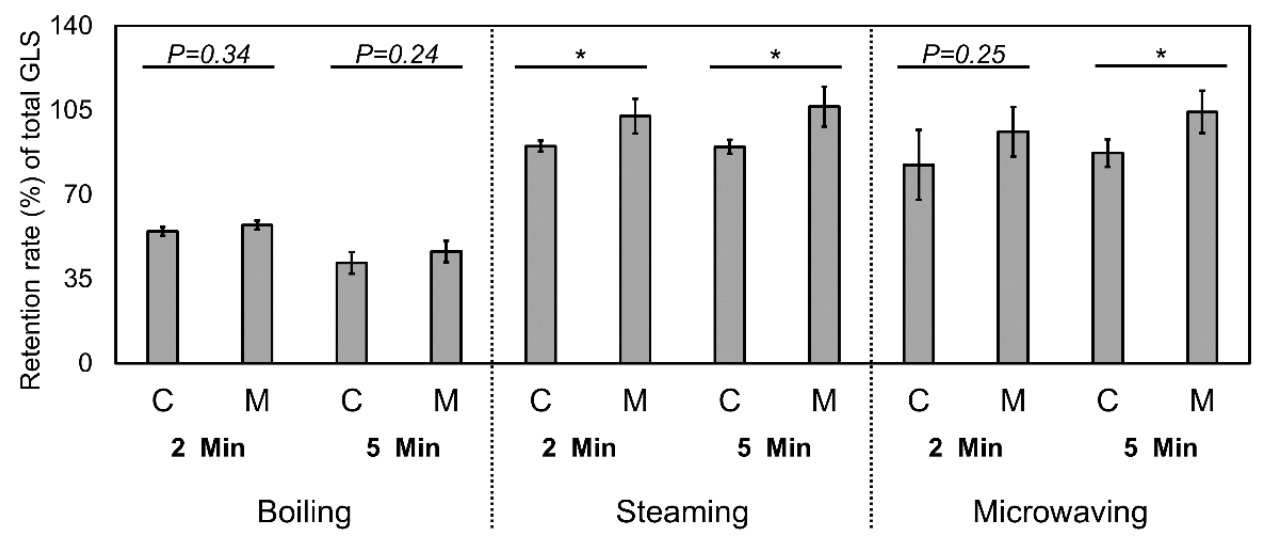

Figure 3.2 Effect of cooking and $250 \mu \mathrm{M}$ MeJA treatment on the retention rate of (A) total aliphatic glucosinolates (GS), (B) total indolyl GS, and (C) total GS.

Asterisk (*) indicates a significant difference compared to the untreated broccoli by Student's ttest at $P \leq 0.05$ based on retention rate $(\mathrm{n}=3)$.

*, $P \leq 0.05$; **, $P \leq 0.01 ; * * *, P \leq 0.001$. 


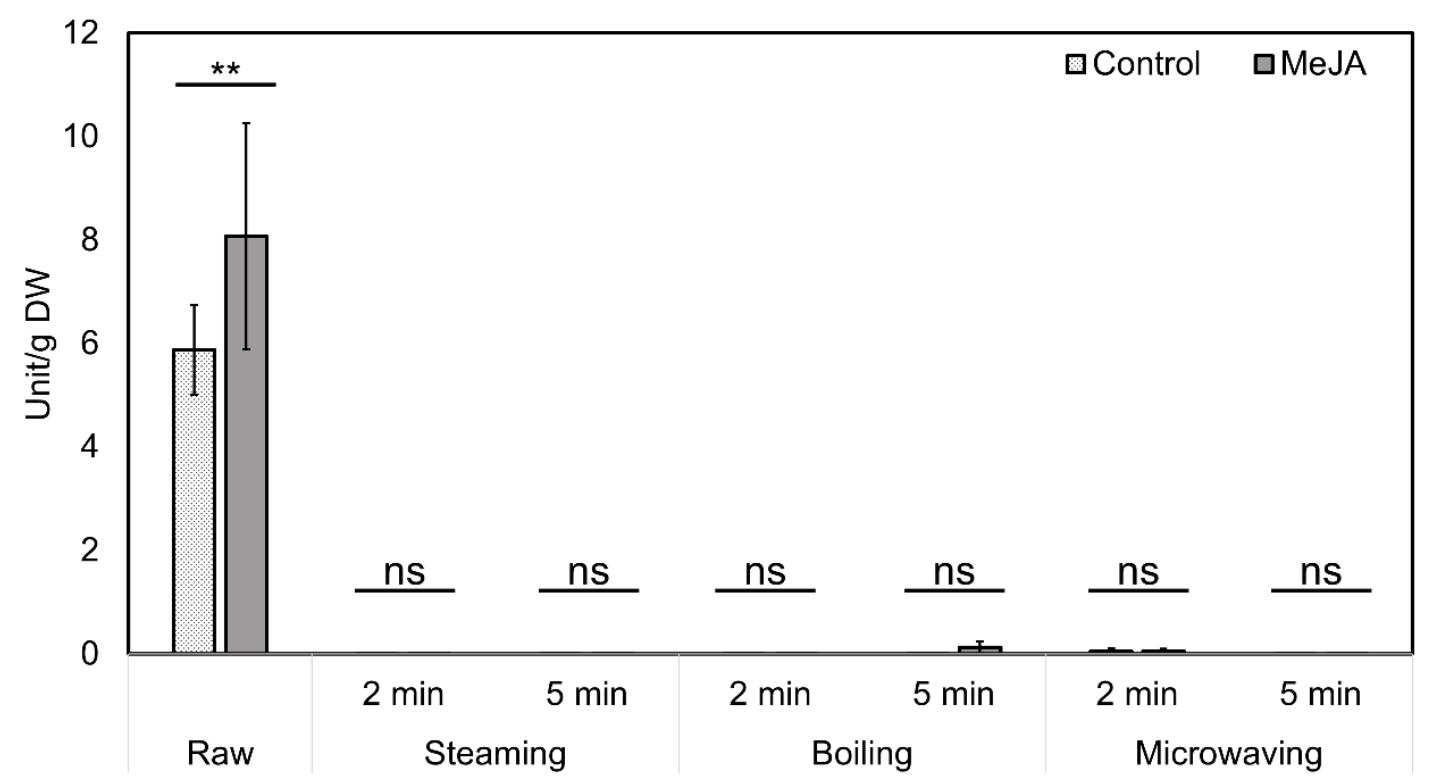

Figure 3.3 Effect of cooking and $250 \mu \mathrm{M}$ MeJA treatment on myrosinase activity in broccoli. Asterisk (*) indicates a significant difference compared to the untreated broccoli by Student's ttest at $P \leq 0.05(\mathrm{n}=3)$.

One unit was defined as $1 \mu \mathrm{mol}$ of total hydrolysis products released per min. ${ }^{\text {ns }}$, not significant; *, $P \leq 0.05 ; * *, P \leq 0.01 ; * * *, P \leq 0.001$. 


\section{Chapter 4 Methyl jasmonate treated broccoli: impact on the production of glucosinolates and consumer preferences ${ }^{3}$}

\subsection{Abstract}

Applying methyl jasmonate can mimic the defense response to insect damage in broccoli and enhances the production of glucosinolates), especially inducible indolyl GS-neoglucobrassicin. Previous studies have suggested that glucosinolates and their hydrolysis products are anticarcinogenic. Therefore, MeJA treatment may increase the nutritional quality of broccoli. However, there are few reports on the sensory evaluation and consumer acceptance of MeJAtreated broccoli. In this study, an untrained consumer panel could not detect any taste differences between steamed MeJA-treated and untreated broccoli, even though the steamed MeJA-treated broccoli contained 50\% more glucosinolates than untreated broccoli. The partial least squareregression model suggested that neoglucobrassicin-derived hydrolysis compounds were the major metabolites that determined overall preference for raw MeJA-treated broccoli potentially due to their potential negative sensory qualities. The results imply that MeJA treatment can increase the nutritional quality of broccoli without sacrificing taste in precooked meals or frozen vegetables.

Keywords: bitter; metabolomics, broccoli, methyl jasmonate, glucosinolate

\footnotetext{
${ }^{3}$ This study is accepted in Food Chemistry.

Chiu, Y. C., Matak, K., \& Ku, K. M. (2019). Methyl jasmonate treated broccoli: Impact on the production of glucosinolates and consumer preferences. Food chemistry, 299, 125099.
} 


\subsection{Introduction}

Brassica vegetables, including broccoli, have been reported to reduce the risk of cancer and cardiovascular disease (Becker \& Juvik, 2016), and this result has been associated with the phytochemicals, glucosinolates (GS) in Brassica vegetables. One of the inducible GS, neoglucobrassicin, can be increased by exogenous applications of methyl jasmonate (Ku et al., 2013; Wiesner et al., 2013). Methyl jasmonate (MeJA) is a signal molecule that is released when a plant is attacked by insects or a physical wound is formed. The jasmonate pathway triggers the plant herbivory defense system and activates a series of physiological events when the plant is subjected to herbivorous attack, and one of these responses is GS biosynthesis (Howe \& Jander, 2008). Studies have shown that the exogenous application of MeJA simulates an insect attack and initiates the jasmonate pathway in plants such as broccoli (Howe \& Jander, 2008).

Many studies have reported that GS hydrolysis products (GSHPs) have health benefits, such as anti-cancer effects (Becker \& Juvik, 2016). However, some GS have been found to be linked to the bitter or pungent taste of Brassicas, and this result may impact consumer preference for these types of vegetables. Sensorial quality also dominates the consumer acceptance of Brassica vegetables (Zabaras et al., 2018). GS themselves cannot fully explain the bitterness associated with Brassica vegetables and the bitterness perception of human is very complex. A recent review suggested that GS hydrolysis products, such as isothiocyanates (ITCs), might contribute more to bitterness perception than glucosinolate itself due to the chemical $\mathrm{N}=\mathrm{C}=\mathrm{S}$ group (thiocyanate moiety) interacting with human bitterness receptors (M. N. Wieczorek et al., 2017). Although thiocyanate moiety from allyl ITC generated the unpleasant taste (Yuesheng Zhang, 2010), sulforaphane, a dominant ITC in broccoli, has thiocyanate moiety were not (Bell, Methven, \& Wagstaff, 2017). Neoglucobrassicin has been associated with bitter taste in cooked 
cauliflower using correlation analysis (Engel, Baty, le Corre, Souchon, \& Martin, 2002b);

however, neoglucobrassicin does not have thiocyanate moiety. Moreover, there was no sensorial quality of neoglucobrassicin-derived hydrolysis reported before. Therefore, it is important to determine the sensorial quality of individual GS to further understand the bitterness of Brassica vegetables.

Presence of sulfur-containing volatiles compounds, including dimethyl disulfide (DMDS) and dimethyl trisulfide (DMTS), might affect the sensorial quality of Brassica as well. The "sulfur odor" in cooked cauliflower has been associated with DMDS and DMTS, and may potentially affect consumer acceptance (Engel et al., 2002b). Exogenous MeJA has been found to induce green leaf volatiles, which is mediated by the lipoxygenase (LOX) biosynthesis pathway and inducing the formation of hexanal and 2-hexanals (Chehab et al., 2008). These volatiles were reported to smell like "fresh cut grass" (hexanal) or "rancid" and "fatty" (2-hexanal), which might influence the sensory evaluation and consumer acceptance of broccoli.

MeJA application can affect postharvest quality and the sensorial quality of various crops (Ku et al., 2013; Luo et al., 2018). However, to our knowledge, there are limited reports on the sensorial quality of MeJA-treated broccoli using consumer panel. In this study, we aimed to understand how consumer acceptance of broccoli related to the phytochemicals and volatiles changes by MeJA treatment. A partial least square-regression (PLS-R) analysis, and an untargeted metabolomic approach were used to narrow down the major compounds that affect consumer preference and to determine the extent to which exogenous MeJA application alters the sensory quality of broccoli. 


\subsection{Materials and methods}

\subsubsection{Broccoli cultivation and sample preparation}

'Broccoli cultivar 'Green Magic' was used because previous study suggested that 'Green Magic' showed similar response to $250 \mu \mathrm{M}$ treatment regardless of years, days from transplant to harvest, accumulated growing degree days, and environmental conditions (Ku, Jeffery, et al., 2013). ‘Green Magic’ broccoli seeds from John's Selected Seeds (Albion, ME, USA) were sown and germinated in trays with Sunshine \#1 Mix (Sun Gro Horticulture, Vancouver, BC, Canada) in the greenhouse facility at West Virginia University for four weeks under a $25 / 18^{\circ} \mathrm{C}$ and $14 / 10$ h day/night temperature/photoperiod regime with supplemental high pressure sodium lighting (600 W HS200 deep reflector; Hortilux, Pijnacker, Netherlands) when the light intensity was below $50 \mathrm{~W} \mathrm{~m}^{-2}$. The seedlings were transplanted into $15 \mathrm{~cm}$ pots for another three weeks and then transplanted to $8.7 \mathrm{~L}$ pots. A total of 40 broccoli heads were harvested 8 weeks later. On the day of the MeJA treatment, half of the broccoli plants were moved outside of the greenhouse for foliage MeJA application. The concentration $(250 \mu \mathrm{M})$ and the timing of MeJA treatment (three to four days prior to the harvest) awas reported to be the most economic-efficient way for increasing overall health-promoting ability of broccoli (Ku et al., 2014b). Before applying MeJA, broccoli head was covered by a sandwich bag and MeJA solution ( $250 \mu \mathrm{M}$ MeJA with $0.1 \%$ Triton X-100; Sigma-Aldrich, St. Louis, MO, USA) was applied to the leaves of the plants. Thirty minutes later, all MeJA-treated broccoli were moved back to the original greenhouse with no excess MeJA solution found on the treated broccoli. All broccoli (control and MeJA-treated broccoli) were harvested three days after MeJA application and stored in separate 18-Gallon tote boxes (Sterilite, Townsend, MA) at $4^{\circ} \mathrm{C}$ refrigerator. After five days, broccoli were used for the sensory evaluation and collected to freeze dry for phytochemical analysis. 
Harvested broccoli were chopped into 3-4 cm diameter florets, and florets were randomized to achieve homogeneity within a treatment group before cooking and serving. Half of the raw florets (both treated and untreated) were placed into 28 gram soufflé cups (plastic serving cups). Then the cups were fitted with lids and identified using random 3-digit number codes. The broccoli florets used in the steamed treatment were evenly spread out without overlapping on a stainless-steel steamer basket, and the steamer basket was placed in a pot over boiling water for 4 minutes. After the steaming process, the florets were transferred to an ice water bath to quick cool them. The chilled steamed florets were then placed into soufflé cups with lids and identified using random 3-digit number codes before serving.

Extra broccoli samples from four treatments (raw control, raw MeJA-treated, steamed control, and steam MeJA-treated) were collected for phytochemical analysis. For each treatment, chopped broccoli florets were randomly distributed into three polyethylene bags as triplicate $(n=3)$ to represent the average of one treatment, and then freeze-dried and ground for further analysis.

\subsubsection{Quantification of glucosinolates}

Glucosinolates were extracted according to Ku et al. (Ku et al., 2016) with slight modifications. Freeze-dried powder was extracted with $70 \%$ methanol at $95^{\circ} \mathrm{C}$ for $10 \mathrm{~min}$. The extract was analyzed on a DEAE Sephadex A-25 (GE Healthcare, Piscataway, NJ, USA). Helix pomatia Type-1 arylsulfatase (Sigma-Aldrich, St. Louis, MO, USA) was added to each column and then incubated for overnight desulfation. The desulfo-glucosinolates were eluted with deionized distilled water and then filtered through a $0.2 \mu \mathrm{m}$ nylon filter. The filtered sample was injected into a Nexera-i, LC 2040C ultrahigh performance liquid chromatograph (UHPLC) (Shimadzu, Kyoto, Japan) equipped with photo diode array (PDA) detector that used a $100 \mathrm{~mm} \times$ 
$2.1 \mathrm{~mm}$ i.d., $1.8 \mu \mathrm{m}, 100 \AA \AA$, Kromasil RP-C18 column (AkzoNobel, Bohus, Sweden). The flow rate was $1 \mathrm{~mL} / \mathrm{min}$ with mobile phase A (deionized distilled water) and B (100\% acetonitrile) with the following elution profile: $0 \min 0 \% \mathrm{~B}, 7 \min 4 \% \mathrm{~B}, 20 \min 20 \% \mathrm{~B}, 35 \min 25 \% \mathrm{~B}, 36$ $\min 80 \% \mathrm{~B}, 40 \mathrm{~min} 80 \% \mathrm{~B}, 41 \mathrm{~min} 0 \% \mathrm{~B}$, and 50 min $0 \% \mathrm{~B}$. Glucosinalbin was used as an internal standard because it was not present in broccoli. For quantification, relative response factor (RRF) of glucosinalbin was set as 1 (Clarke, 2010). The UV response factors for various glucosinolates (Kusznierewicz et al., 2013) applied for quantification were: glucoiberin 1.07, progoitrin 1.09, glucoraphanin 1.07, sinigrin 1.00, gluconapin 1.11, glucoerucin 1.00, glucobrassicin 0.29, 4-hydroxyglucobrassicin 0.28, 4-methoxyglucobrassicin 0.25 , gluconasturtiin 0.95 , and neoglucobrassicin 0.20 . RRF of 1-hydroxyglucobrassicin was set as 0.28 (similar structure to 4-hydroxyglucobrassicin) because RRF was not available.

Freeze-dried broccoli powder (50 mg) was suspended in $1 \mathrm{~mL}$ distilled water in a $2 \mathrm{~mL}$ microcentrifuge tube (Fisher Scientific, Waltham, MA, USA). The hydrolysis products were generated naturally by endogenous myrosinase in the absence of light at room temperature for 24 h. Then $1 \mathrm{~mL}$ of dichloromethane was added and the samples were centrifuged at $12,000 \times g$ for $2 \mathrm{~min}$. The lower organic layer was collected and analyzed using a gas chromatograph (Trace 1310 GC, Thermo Fisher Scientific, Waltham, MA, USA) coupled to an MS detector system (ISQ QD, Thermo Fisher Scientific, Waltham, MA, USA) and an autosampler (Triplus RSH, Thermo Fisher Scientific, Waltham, MA, USA). A capillary column (Rxi-5Sil MS, Restek, Bellefonte, PA, USA; $30 \mathrm{~m}, 0.25 \mathrm{~mm}, 0.25 \mathrm{~m}$, capillary column w/10 m Integra-Guard Column) was also used. The peaks were identified using information from a previous publication and by comparing them with data in the National Institute of Standards and Technology (NIST) library.

\subsubsection{Quantification of myrosinase activity and nitrile formation}


Published methods (Kim, Chiu, \& Ku, 2017) were used to measure the myrosinase activity and the percentage nitrile formation to total hydrolysis products from glucosinolate ratio. These values were then used to estimate epithiospecifier protein (ESP) levels and the epithiospecifier modifier 1 (ESM1) interaction. Myrosinase activity was estimated as the total amount of hydrolysis products produced within $60 \mathrm{~min}$. One unit was defined as $1 \mu \mathrm{mol}$ of total hydrolysis products released per minute. Nitrile formation was determined by incubating concentrated horseradish root extract with protein extracts from broccoli samples. The horseradish extract was prepared and used as an exogenous substrate source of sinigrin and gluconasturtiin at a saturated level to minimize of the reaction with endogenous glucosinolate substrates from the different broccoli samples (Ku, Jeffery, Juvik, \& Kushad, 2015). The hydrolysis products from sinigrin and gluconasturtiin were the dominant compounds detected by GC-MS. A freeze-dried powdered sample $(75 \mathrm{mg})$ was mixed with $1.5 \mathrm{~mL}$ of concentrated horseradish root extract in $2 \mathrm{~mL}$ microcentrifuge tubes. After centrifugation at $12,000 \times g$ for 2 min, $0.5 \mathrm{~mL}$ of the supernatant was transferred to a $1.5 \mathrm{~mL}$ Teflon centrifuge tube (Savillex Corporation, Eden Prairie, MN, USA) and $0.5 \mathrm{~mL}$ of dichloromethane was added. The tube was then placed upside down at room temperature for 10 min to minimize volatile compound losses. Then, the tube was vortexed and centrifuged at $12,000 \times g$ for $4 \mathrm{~min}$. The dichloromethane organic layer was injected into the GC/MS system described above to determine the GSHPs. After an initial temperature hold at $40^{\circ} \mathrm{C}$ for $2 \mathrm{~min}$, the oven temperature was increased to $320^{\circ} \mathrm{C}$ at $15^{\circ} \mathrm{C} \mathrm{min}-1$ and held for $4 \mathrm{~min}$. The injector and detector temperatures were set at $270^{\circ} \mathrm{C}$ and $275^{\circ} \mathrm{C}$, respectively. The flow rate of the helium carrier gas was set at $1.2 \mathrm{~mL} \mathrm{~min}^{-1}$. The standard curves for allyl isothiocyanate, 2-phenethyl isothiocyanate, and 3-phenylpropionitrile 
(Sigma-Aldrich, St Louis, MO, USA) were used for quantification. The standard curve for allyl isothiocyanate was used to quantify 1-cyano-2,3-epithiopropane.

\subsubsection{Volatile organic compounds (VOCs) profiling by in-tube extraction (ITEX)}

The GC coupled with the autosampler and ITEX tool was used to collect the volatile organic compounds (VOCs) released by the lyophilized broccoli sample in the headspace. Briefly, a 0.2 $\mathrm{g}$ broccoli sample was mixed with $10 \mathrm{~mL}$ of water in a $20 \mathrm{~mL}$ headspace sample vial. The sample vial was transferred to the agitator and was agitated at $65^{\circ} \mathrm{C}$ for 20 mins. After incubation, the needle of the syringe was introduced into the headspace of the vial and the syringe extracted the gaseous VOCs via a porous adsorption polymer fiber polydimethylsiloxane (PDMS) microtrap (ITEX-2, Switzerland), and PDMS is common resin used for Brassica vegetables (Wieczorek \& Jeleń, 2019). The extraction was repeated 40 times. The samples, which had undergone thermal desorption, were then directly injected into the GC-MS and cleaned with a hot $\operatorname{trap}\left(250^{\circ} \mathrm{C}\right)$.

The extracted volatiles were separated by the same GC-MS used in the previous section (section 2.3). The carrier gas was helium with flow rate $1.2 \mathrm{~mL} \mathrm{~min}^{-1}$ and splitless injection was employed. The injection temperature was $250^{\circ} \mathrm{C}$. The column oven was kept at $50^{\circ} \mathrm{C}$ for 2 min

and increased to $250^{\circ} \mathrm{C}$ at $12^{\circ} \mathrm{C} \mathrm{min}^{-1}$ and held for $2 \mathrm{~min}$. The ion-source was kept at $250^{\circ} \mathrm{C}$ and the interface temperature was $300^{\circ} \mathrm{C}$. The mass spectrometer was operated in positive electron impact mode (EI) at $70.0 \mathrm{eV}$ ionization energy within an $\mathrm{m} / \mathrm{z} 45-350 \mathrm{scan}$ range. The compounds were identified using the NIST library or by available authentic standards (Table S4).

\subsubsection{Untargeted primary metabolites identification by GC-MS}


Primary metabolites were extracted using published protocols (Lisec et al., 2006) with modifications. The samples $(50 \mathrm{mg}$ ) were placed in $2 \mathrm{~mL}$ microcentrifuge tubes. Ribitol was added as an internal standard and then the primary metabolites were extracted with $1.4 \mathrm{~mL}$ of methanol at $75^{\circ} \mathrm{C}$. After cooling, the sample extracts were centrifuged and the supernatant was transferred to new $2 \mathrm{~mL}$ microcentrifuge tubes. Then $0.375 \mathrm{~mL}$ of cold chloroform $\left(-20^{\circ} \mathrm{C}\right)$ and $0.7 \mathrm{~mL}$ cold water $\left(4^{\circ} \mathrm{C}\right)$ were added to fractionate the polar compounds. After vigorous mixing, the extracts were centrifuged at $12,000 \mathrm{~g}$ for $5 \mathrm{~min}$ and transferred to $1.5 \mathrm{~mL}$ tubes. The extracts were dried using a VacufugeTM concentrator (Eppendorf, Thermo Fisher Scientific, Waltham, MA, USA). The dried extracts were derivatized with methoxyamine hydrochloride for $90 \mathrm{~min}$ at $37^{\circ} \mathrm{C}$ and then with $70 \mu \mathrm{L}$ MSTFA $+1 \%$ TMCS at $37^{\circ} \mathrm{C}$ for $30 \mathrm{~min}$. The metabolites were analyzed using a GC-MS with an autosampler. The mass spectrometer was operated in positive electron impact mode (EI) at $70.0 \mathrm{eV}$ ionization energy within an $\mathrm{m} / \mathrm{z} 40-500$ scan range.

The acquired chromatograms were converted to mzXML using RawConverter (He et al., 2015). Peak detection and alignment was performed by the XCMS package in $R$ language with the default settings. All data was normalized to the unique ion peak $(319 \mathrm{~m} / \mathrm{z})$ produced by the internal standard using the online platform MetaboAnalyst (Chong et al., 2018). Metabolite identification was based on authentic standard compounds (STD) and then compared with the mass spectra present in NIST library and their retention times (Table S5).

\subsubsection{Consumer-based sensory evaluation}

The sensory evaluation and the questionnaire designed for panelist was approved by the Institutional Review Board at WVU. Panelists were recruited during 2017 summer by flyers in the building and in person invitations at Davis College of West Virginia University; recruitment was not limited to Davis College colleagues. All panelists participated voluntarily, and no 
incentives were provided. Participants were given a short presentation prior to testing and then they filled out a demographic questionnaire. We recruited 60 consumers and the ratio of female to male was 6:4. Among all panelists, $73 \%$ of the panelists were between $18-35$ years old; $10 \%$ of the panelists were between 36-54 years old, and the rest of the panelists (17\%) were older than 55.

After the panelists completed the questionnaire, they were then led to the sensory evaluation room. Each participant was then involved in three types of test: (1) triangle test, (2) attribute test, and (3) preference test. Each test was conducted two times; first test was used steamed broccoli (milder taste) and the second test was used raw broccoli (stronger taste) to avoid sensory fatigue. Each sample has its unique 3-digit code when presented to panelists. The samples were presented in a completely random blocked design and the results were collected using a printed evaluation card. Illegible questionnaires were removed before statistical analysis and therefore the sample size sometimes changed. The triangle test results were analyzed by Chi-square test $(P \leq 0.05)$. The attribute test and preference test results were analyzed by Student's $t$-test $(P \leq 0.05)$.

In the first triangle test, panelists were first presented with three steamed broccoli samples. Half of the panelists received two raw control broccoli and one raw MeJA-treated broccoli while the other half of the panelists received two raw MeJA-treated broccoli and one raw control broccoli. The panelists were asked to choose the different sample out of the three only based on flavor. The panelists were also asked to describe the taste of the unique sample, if possible. After the first test, panelists were presented with second triangle test. All designs remained the same except the material (steamed broccoli) was different.

In the first attribute test and preference test, the panelists were presented with a set of two broccoli (steamed control and MeJA-treated broccoli) and asked to rate appearance and color, 
taste and flavor, texture and mouthfeel, and overall liking on a 7-point hedonic scale for both. Panelists were then asked to choose the preferred sample out of two. After the first test, panelists were presented with second set of samples. All designs remained the same except the material (raw broccoli) was different.

\subsubsection{Univariate and multivariate analyses}

The statistical analyses (Student's $t$-test, two-way univariate analysis of variance (ANOVA), Tukey's HSD, and partial least square-regression (PLS-R)) were conducted using JMP 14 (SAS Institute, Cary, NC, USA). The significance level was set at $P \leq 0.05$ for all tests. The primary metabolites statistical analysis was conducted using the MetaboAnalyst online platform.

\subsection{Results and discussions}

4.4.1 Quantification of glucosinolate concentration and abundance of glucosinolate hydrolysis products in 'Green Magic' broccoli

The total GS in the raw control broccoli was $8.05 \mu$ mole $\cdot g^{-1}$ dry weight (DW) with $90 \%$ water content of raw material. However, MeJA-treated broccoli contained a significantly greater total GS concentration $\left(12.34 \mu\right.$ mole $\left.\cdot \mathrm{g}^{-1} \mathrm{DW}\right)$, which was an increase of $50 \%$ (Table 1$)$. The neoglucobrassicin concentration in raw MeJA-treated broccoli increased 8-fold compared to the raw broccoli control samples. This result was consistent with previous studies, which showed that the neoglucobrassicin concentration increases were mainly due to the MeJA treatment (H. S. Kim \& Juvik, 2011). Total aliphatic GS in the raw broccoli control sample was $5.3 \mu \mathrm{mole} \cdot \mathrm{g}^{-1}$ DW, whereas in MeJA-treated raw broccoli, the concentration significantly decreased to 3.97 $\mu$ mole $\cdot \mathrm{g}^{-1} \mathrm{DW}(P \leq 0.05)$. This decrease may potentially due to the higher degradation rate of glucoraphanin in MeJA-treated broccoli during postharvest period (Ku et al., 2013). 
Total GS levels significantly decreased in both samples after steaming (Jones, Frisina, Winkler, Imsic, \& Tomkins, 2010). Four minutes steaming treatment decreased total GS by $13 \%$ in the control broccoli (6.96 $\mu$ mole $\left.\cdot \mathrm{g}^{-1} \mathrm{DW}\right)$ and by $15 \%$ in MeJA-treated broccoli (10.44 $\mu$ mole. $\left.\mathrm{g}^{-1} \mathrm{DW}\right)$. After steaming, MeJA-treated broccoli still had significantly more total glucosinolates than the untreated broccoli $(P \leq 0.05)$, which means the health benefits derived from the glucosinolates in MeJA-treated broccoli may still be utilized in diet because human microbiome may digest intact GS (Tian, Liu, Lei, Zhang, \& Shan, 2018).

Fourteen GSHPs were detected in the raw broccoli, including isothiocyanates (ITCs), nitriles, indoles, and oxazolidine-thione. However, only six different GSHPs were detected in the steamed broccoli samples (Figure 3.1; Supplementary Table S6). The MeJA treatment significantly increased the amount of neoglucobrassicin (Table 3.1), and the hydrolysis products derived from neoglucobrassicin ( $N$-methoxyindole-3-carbinol (NMI3C), $N$-methoxyindole-3carboxyaldehyde (NMI3CA), and $N$-methoxyindole-3-acetonitrile(NMI3ACN)) were also significantly higher in raw MeJA-treated broccoli. This result agreed with previous research on broccoli (Ku, Becker, \& Juvik, 2016) and 'kale (Chiu et al., 2018). Although sulforaphane was known for its high bioactivity, the correlation coefficient between quinone reductase (a phase II detoxification enzyme for carcinogens) inducing activity and sulforaphane was similar to indole3-carbinol (I3C) (Ku et al., 2013), which has similar chemical structure to NMI3C. It was also reported that NMI3C is a stronger prohibitor of cytochrome P450 1A1 (involved in tumor development) than I3C (Stephensen et al., 2000). Furthermore, the bio activity of sulforaphane and I3C was dose-dependent (Frydoonfar, McGrath, \& Spigelman, 2003), which implies that higher concentration of hydrolysis products and the precursor GS may show stronger bioactivity. 
Therefore, the increase of neoglucobrassicin and its hydrolysis products may increase the nutritional quality of broccoli.

\subsubsection{Myrosinase activity and nitrile formation in 'Green Magic' broccoli}

Myrosinase activity was measured to determine whether GSHP formation preference was altered by MeJA treatment and the steaming process. In raw broccoli, myrosinase activity in the

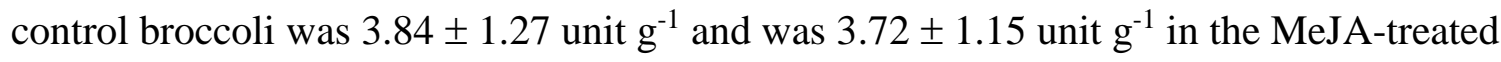
broccoli. Therefore, the $250 \mu \mathrm{M}$ MeJA treatment did not change $(P=0.90)$ the myrosinase activity in the raw broccoli (Figure S3).

Nitrile formation was determined from the ratio of the concentration of nitriles to the total concentration of the hydrolysis products formed (sum of isothiocyanates and nitriles) from sinigrin and gluconasturtiin. A high value indicates that more nitriles are formed compared to ITCs. The nitrile formation value can be used as an indirect method to measure ESP activity (Kim et al., 2017). There was a significant decrease $(P \leq 0.05)$ of nitrile formation from gluconasturtiin in the raw MeJA-treated broccoli compared to the control (Figure S1), but there was no significant difference $(P=0.17)$ in nitrile formation from sinigrin (Figure $\mathrm{S} 1)$. The discrepancy might be due to the affinity of broccoli myrosinase for different substrates or different binding sites (Román, Castillo, Cottet, \& Mahn, 2018). This result was different from the results for pak choi (Kim et al., 2017) and 'Red Russian' kale (Chiu et al., 2018) where it was the leaf tissue that was harvested and analyzed rather than a floret. In the pak choi study, the authors also found the response to MeJA treatment was cultivar-specific. In this study, the MeJA solution was applied to broccoli leaves and the broccoli florets were harvested as the experiment material rather than the leaves. Furthermore, broccoli belongs to the species Brassica oleracea 
while pak choi and 'Red Russian' kale belong to Brassica rapa and Brassica napus, respectively. Different genetic backgrounds will potentially change their responses. In this study, erucin nitrile, iberin nitrile, and sulforaphane nitrile were significantly lower in raw MeJA-treated broccoli $(P \leq 0.05)$. Taken this result together with higher GS amount in MeJA-treated broccoli, the results implied that the nutritional quality of the MeJA-treated broccoli may have been improved because ITCs have been shown to have a higher bioactivity than nitriles (Matusheski \& Jeffery, 2001).

After the 4 min steaming process, myrosinase activity was significantly reduced $(P \leq 0.05)$

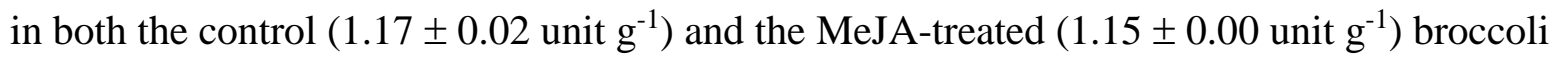
(Figure 2A) compared to the raw samples. This result corresponded well with the significant GSHPs decrease $(P \leq 0.05)$ in both the steamed control and the steamed MeJA-treated broccoli (Table S6). Steaming deactivated myrosinase and therefore the GS hydrolysis process was significantly reduced $(P \leq 0.05)$, which led to fewer detectable GSHPs in the steamed broccoli samples (Table S6). In this study, broccoli was steamed for 4 min, which was probably sufficient to increase the inner temperature of the floret to above $100^{\circ} \mathrm{C}$ (Sarvan, Kramer, Bouwmeester, Dekker, \& Verkerk, 2017). Myrosinase is deactivated at temperatures above $80^{\circ} \mathrm{C}$ (R. Björkman \& Lönnerdal, 1973). Therefore, it was not surprising to detect significantly lower GSHP concentrations $(P \leq 0.05)$ in the steamed samples.

\subsubsection{Measurement of polar primary metabolites using an untargeted metabolomic approach}

Although the effect of MeJA application on untargeted metabolomic data in Brassica vegetables have been analyzed before (Chiu et al., 2018; Kim et al., 2017; Ku, Becker, \& Juvik, 2016), the investigation on broccoli is still limited. Among the polar metabolites, eight amino acids, three organic acids, two sugars, and one sugar alcohol were found to be significantly 
different by the two-way ANOVA at $P \leq 0.05$ (Figure 3.2). In the partial least square discriminant analysis (PLS-DA), metabolites with an Importance in the Projection (VIP) value > 1.5 can be considered as potential biomarkers. Within the four groups (raw control, raw MeJA, steamed control, and steamed MeJA), metabolites such as glucose, myo-inositol, alanine, fructose, glutamine, glutamic acid, and malic acid were significantly reduced $(P \leq 0.05)$ in steamed broccoli (Figure 2; Supplementary Table S5). The amino acid, organic acid, and sugar levels were significantly higher $(P \leq 0.05)$ in raw broccoli, which indicated that the steaming process may lead to the loss of primary metabolites in broccoli. All the biomarkers were used in the projection model to determine their contribution to the sensory evaluation results.

MeJA treatment had no significant effect on polar metabolites in either the raw samples or the steamed samples, which volcano plots implied (Figure S4). It was reported that MeJA treatment may reduce sugars by simulating the effect of herbivore attack on a plant (Chiu et al., 2018). The sugar content usually significantly lower in some MeJA-treated pak choi cultivars; however, the effect was cultivar-dependent (Kim et al., 2017). Therefore, genetic background may also alter the effect of MeJA application on primary metabolites in Brassica vegetables. The non-significant effect of the MeJA treatment on raw and steamed broccoli also suggested that the detected polar primary metabolites may not lead to sensorial quality change in broccoli (section 4.4.5).

\subsubsection{In-tube extraction (ITEX) analysis of fresh broccoli samples}

The volatile profiles of the control and MeJA-treated broccoli were obtained using the ITEX/GC-MS technique in order to gain a better understanding of whether the MeJA treatment affected the volatile compound profile, and whether the volatile compound profile affected the results of the sensory evaluation. A total of eight major volatile compounds were detected and 
identified (Table S1). These were dimethyl sulfide, 2-ethylfuran, dimethyl disulfide, hexanal, (E)-2-hexanal, dimethyl trisulfide, nonanal, and dimethyl tetrasulfide after using PDMS resin (Table 3.2).

The major volatiles identified in the raw control broccoli were sulfur volatiles (dimethyl sulfide, dimethyl disulfide, dimethyl trisulfide, and dimethyl tetrasulfide), which made up of 98.3\% of all detected volatiles (Table 2). In raw MeJA-treated broccoli, sulfur volatiles only accounted for $58.1 \%$ of the detectable volatiles. Sulfur-containing volatiles, including dimethyl disulfide and dimethyl trisulfide, are common volatiles in brassica vegetables and usually present a "sulfur-like", "cabbage like", or "spoiled cabbage" smell to the consumer (Wieczorek et al., 2017). However, the formation of sulfur-containing volatiles in MeJA-treated broccoli was significantly reduced $(P \leq 0.05)$. The enzyme required for dimethyl sulfides formation (cysteine sulfoxide lyase) and the substrate, $S$-methyl-L-cysteine sulfoxide (SMCSO) may have been affected by MeJA treatment. Because both SMCSO and glucosinolates are sulfur-containing compounds, the difference could be due to the repartitioning of sulfur or sulfur assimilation (Traka et al., 2013), which suggests that the MeJA treatment may be involved in this change.

The MeJA treatment caused a significant increase $(P \leq 0.05)$ in the formation of aldehyde volatiles (hexanal, (E)-2-hexanal, and nonanal), which made up 35.1\% of the total volatiles (Table 2). It has been reported that the increased formation of aldehydes by exogenous MeJA application in barley leaves (Kohlmann et al., 1999) and cucumber (Jiang et al., 2017). Interactions between jasmonates and aldehydes (LOX-pathway volatiles such as hexanals) have also been reported to play a critical role in overall plant-defense responses (Chehab et al., 2008). The exogenous application of MeJA activates the defense system in a similar way to a generalist 
attack on kale (Chiu et al., 2018). Therefore, the increased hexanal levels in MeJA-treated broccoli recorded by this study were consistent with previous reports.

After steaming, volatile abundance in the control and MeJA-treated broccoli significantly decreased (Figure S5), which was consistent with previous research (Wieczorek \& Jeleń, 2019). Inactivation of cysteine sulfoxide lyase may occur during the steaming process and could have led to the overall lower volatile abundance in both samples.

\subsubsection{Results from the sensory evaluation}

The triangle test is used to determine whether the customer panel can detect the odd sample in three provided samples (Meilgaard, Carr, \& Civille, 2006). In this study, 20 out of 60 panelists correctly selected the odd samples based on flavor in steamed broccoli triangle test, which meant no significant difference by Chi-square test $(P=0.99)$ between the steamed control and MeJAtreated broccoli. In contrast, 32 out of 60 panelists correctly detected the odd samples from raw broccoli triangle test, which implied a significant difference between untreated and MeJA-treated broccoli by Chi-square test at $P \leq 0.05$.

In the steamed broccoli preference test, panelists $(n=56)$ showed no preference difference between the control and MeJA-treated broccoli, but they significantly $(P \leq 0.05)$ preferred the raw control broccoli over the raw MeJA-treated broccoli. These results are consistent with the triangle test and support the theory that steaming will reduce detectable differences between the samples and consumer panelists could not tell the difference between MeJA-treated broccoli and regular broccoli if broccoli is cooked.

The attribute test results for steamed broccoli showed that there were no significant differences in appearance and color $(P=0.92)$, taste and mouth feel $(P=0.24)$, texture and 
mouth feel $(P=0.26)$, and overall liking $(P=0.41)$ between the steamed control broccoli and MeJA-treated broccoli (Table 3). In contrast, the attribute rating results for raw broccoli $(\mathrm{n}=58)$ were significantly changed $(P \leq 0.05)$ by $250 \mu \mathrm{M}$ MeJA treatment (Table 3$)$. Raw MeJA-treated broccoli was significantly less accepted $(P \leq 0.05)$ by the consumer panelists compared to the raw control broccoli except for outer appearance. Panelists described the undesirable flavors in the MeJA-treated broccoli as "grassy", a "horrible taste", and a "strong aftertaste". Some feedback also stated that the raw MeJA-treated broccoli "tasted a little more bitter". Results from sensory evaluation showed that 4 minutes steaming reduced the detectable differences in MeJAtreated broccoli for untrained panelists, which was more applicable to regular consumers.

Flavor and aroma are two key factors that critically affect repeated purchase behavior (Garitta, Hough, \& Chaves, 2013). Evidence (Bongoni, Verkerk, Steenbekkers, Dekker, \& Stieger, 2014; Garitta et al., 2013) suggested that most consumers often cook broccoli prior to consumption. Based on our results, the attribute evaluation of raw broccoli indicated that the actual taste of raw MeJA-treated broccoli might not be acceptable to consumers. However, the detectable difference by MeJA treatment might be negligible after steaming according to the results produced by this study, especially when broccoli is mostly eaten cooked. It was suggested that $0.5 \mu \mathrm{M}$ MeJA treatment increased the sweetness and decreased the bitterness of broccoli using electronic tongue (Luo et al., 2018); therefore, the different application concentration of MeJA may also alter the sensorial quality of broccoli.

\subsubsection{Partial least square-regression analysis results suggested that neoglucobrassicin played a role in driving consumer overall liking for 'Green Magic' broccoli}

To identify the major compounds that affected consumer overall liking and the taste/flavor of broccoli, a partial least square-regression (PLS-R) analysis was conducted on the results of 
sensory evaluation (overall liking and taste and flavor) and metabolite profiles (nine GS, 16 GSHPs, eight volatile compounds, and 14 primary metabolites with VIP value $>1.5$ by PLS-DA in a non-linear iterative partial least squares (NIPALS) algorithm after centering and scaling the data using JMP. A "Leave-One-Out" statistical approach was used for cross validation in the PLS model. The overall liking prediction model derived from the PLS-R analysis showed that the root-mean-square-error (RMSE) of the fit for collected overall liking was 0.05 . The correlation between predicted overall liking and measured overall liking was highly correlated $\left(\mathrm{R}^{2}=0.97\right)$ with two of the extracted factors (Figure S6). Among all metabolites with VIP value above 1.5 calculated by the model, NMI3ACN, NMI3C, and NMI3CA (hydrolysis products from neoglucobrassicin), brassicanapin, volatiles (E)-2-hexanal, 4-methoxy-glucobrasscin, 2-ethylfuran, glucoerucin, and I3C (Table 3.4). When the same variables in the overall liking model were used to test on the prediction model of "taste and flavor", the model showed a RMSE of 0.05 and the correlation between predicted taste and flavor score and measured taste and flavor score were highly correlated $\left(\mathrm{R}^{2}=0.98\right)$ with three extracted factors (Figure S6). In this model, metabolites with VIP value above 1.5 were the same as in overall liking model (Table 3.4).

To our knowledge, this study may be the first report on the potential sensorial quality of neoglucobrassicin-derived hydrolysis products. The results of PLS-R model showed that neoglucobrassicin-derived hydrolysis products (NMI3ACN, NMI3C, and NMI3CA) significantly contributed to the taste and the flavor, and they also showed higher VIP value than neoglucobrassicin itself in overall liking model. Considering the unpleasant taste of raw MeJAtreated broccoli reported by the panelists, it is possible that neoglucobrassicin-derived hydrolysis products contributed to taste and flavor in raw MeJA-treated broccoli. In this study, the role of these hydrolysis products may be as important as the precursor (neoglucobrassicin) when 
attempting to determine the sensorial quality of broccoli because these products had very high VIP values in the taste and flavor model and in the overall liking model. Therefore, neoglucobrassicin-derived hydrolysis products may not only be significant metabolites in determining overall liking by the PLS-R model, but also potentially contributed to the detectable taste of raw MeJA-treated broccoli.

\subsection{Conclusion}

The results indicated that steaming can effectively eliminate the detectable difference by MeJA treatment in broccoli to the untrained panelists. MeJA-treated broccoli can be especially useful in prepared food that contains precooked or frozen broccoli for food industry as a valueadded component in prepared meals or frozen vegetables. After 4 min of steaming, the taste of control and MeJA-treated was the same to the untrained panelists, while the total GS levels in MeJA-treated broccoli were still 50\% higher than in the untreated broccoli. In addition, neoglucobrassicin-derived hydrolysis products played an important role in sensory quality of broccoli determined by the PLS-R model. The results also help to explain the unpleasant taste of broccoli when they are attacked by herbivores in the field because MeJA treatment simulates a plant-herbivore defense response. This study may be the first to use a consumer panel survey to evaluate the sensory quality of MeJA-treated broccoli, and the results also provide valuable information about applying MeJA as part of Brassica crop agricultural regimes.

\section{Conflict of interest}

The authors declare no conflict of interest with regards to this work.

\section{Acknowledgments}


This study is based on research that is supported by the National Institute of Food and Agriculture, U.S. Department of Agriculture, Hatch project number \#WVA 00722 and 112506. 
Table 4.1. Glucosinolate profile $\left(\mu \mathrm{mole} \cdot \mathrm{g}^{-1} \mathrm{DW}\right)$ of raw and steamed broccoli with or without $250 \mu \mathrm{M}$ methyl jasmonate application (average $\pm \mathrm{SD}$ ).

\begin{tabular}{lcccc}
\hline \multirow{2}{*}{ Glucosinolates } & \multicolumn{2}{c}{ Raw } & \multicolumn{2}{c}{ Steamed } \\
\cline { 2 - 5 } & \multicolumn{1}{c}{ Control } & \multicolumn{1}{c}{ MeJA } & Control & MeJA \\
\hline Aliphatic GS & & & & \\
Glucoiberin & $0.91 \pm 0.11$ & $0.72 \pm 0.03$ & $0.76 \pm 0.15$ & $0.63 \pm 0.12$ \\
Progoitrin & $0.45 \pm 0.03$ & $0.36 \pm 0.01 *$ & $0.36 \pm 0.01 \mathrm{~b}^{\mathrm{z}}$ & $0.30 \pm 0.06$ \\
Glucoraphanin & $3.52 \pm 0.43$ & $2.62 \pm 0.12 *$ & $2.93 \pm 0.55$ & $2.29 \pm 0.50 *$ \\
Sinigrin & $0.17 \pm 0.01 \mathrm{a}$ & $0.10 \pm 0.01 *$ & $0.14 \pm 0.01 \mathrm{~b}$ & $0.08 \pm 0.02 *$ \\
Gluconapin & $0.05 \pm 0.00 \mathrm{a}$ & $0.02 \pm 0.00 *$ & $0.04 \pm 0.00 \mathrm{~b}$ & $0.02 \pm 0.01 *$ \\
Glucoerucin & $0.20 \pm 0.02$ & $0.15 \pm 0.01 *, \mathrm{~b}$ & $0.22 \pm 0.02$ & $0.18 \pm 0.02 *, \mathrm{a}$ \\
Total aliphatic GS & $5.30 \pm 0.58$ & $3.97 \pm 0.14 *, \mathrm{a}$ & $4.45 \pm 0.73$ & $3.50 \pm 0.71 *, \mathrm{~b}$ \\
$\begin{array}{l}\text { Indolyl GS } \\
\text { Glucobrassicin }\end{array}$ & $0.70 \pm 0.03$ & $0.79 \pm 0.00 *$ & $0.63 \pm 0.05 \mathrm{~b}$ & $0.74 \pm 0.08 *$ \\
4-Methoxy- & $0.23 \pm 0.01 \mathrm{a}$ & $0.33 \pm 0.02 *$ & $0.19 \pm 0.02 \mathrm{c}$ & $0.28 \pm 0.01 *$ \\
glucobrassicin & & & & \\
Neoglucobrassicin & $0.76 \pm 0.03$ & $6.56 \pm 0.72 *, \mathrm{a}$ & $0.59 \pm 0.04 \mathrm{c}$ & $5.22 \pm 0.14 *, \mathrm{~b}$ \\
1-Hydroxy- & $1.03 \pm 0.10$ & $0.56 \pm 0.72 *$ & $1.07 \pm 0.04 \mathrm{a}$ & $0.61 \pm 0.14 *$ \\
glucobrassicin & & & & \\
Total indolyl GS & $1.68 \pm 0.06$ & $7.68 \pm 0.74 *$ & $1.41 \pm 0.10 \mathrm{c}$ & $6.23 \pm 0.22 *$ \\
Gluconasturiin & $0.04 \pm 0.00$ & $0.14 \pm 0.02 *$ & $0.03 \pm 0.00 \mathrm{c}$ & $0.11 \pm 0.03 *$ \\
Total GS & $8.05 \pm 0.68$ & $12.34 \pm 0.91 *, \mathrm{a}$ & $6.96 \pm 0.83 \mathrm{c}$ & $10.44 \pm 1.05 *, \mathrm{~b}$ \\
\hline
\end{tabular}

* Asterisk indicates significant difference before and after steaming within the same treatment group by Student's T-test $(P \leq 0.05, N=3)$.

${ }^{\mathrm{z}}$ Different letters indicate significant difference with or without MeJA treatment within the same cooking treatment group by Student's T-test $(P \leq 0.05, N=3)$ 
Table 4 2. Volatile composition of raw and steamed 'Green Magic' broccoli with or without 250 $\mu \mathrm{M}$ methyl jasmoante treatment.

\begin{tabular}{|c|c|c|c|c|}
\hline \multirow{2}{*}{ Percentage $(\%)^{\mathrm{z}}$} & \multicolumn{2}{|c|}{ Raw } & \multicolumn{2}{|c|}{ Steamed } \\
\hline & Control & MeJA & Control & MeJA \\
\hline Dimethyl sulfide & $24.93 \pm 0.24 \mathrm{a}$ & $42.41 \pm 5.12 *, \mathrm{a}$ & $91.28 \pm 3.92 b$ & $91.82 \pm 0.55 b$ \\
\hline Dimethyl tisulfide & $7.13 \pm 0.88 \mathrm{a}$ & $2.79 \pm 0.68 *, \mathrm{a}$ & $0.49 \pm 0.10 b$ & $0.19 \pm 0.05 b$ \\
\hline Dimethyl trisulfide & $62.67 \pm 1.01 \mathrm{a}$ & $12.86 \pm 4.57^{*}, \mathrm{a}$ & $0.09 \pm 0.15 b$ & $0.02 \pm 0.03 \mathrm{~b}$ \\
\hline Dimethyl tetrasulfide & $3.55 \pm 0.22 \mathrm{a}$ & $0.00 \pm 0.00 *$ & $0.01 \pm 0.02 \mathrm{~b}$ & $0.01 \pm 0.01$ \\
\hline $\begin{array}{c}\text { Total sulfides } \\
\text { volatiles }\end{array}$ & $98.27 \pm 0.43$ & $58.05 \pm 10.36 *, a$ & $91.87 \pm 4.06$ & $92.04 \pm 0.50 \mathrm{~b}$ \\
\hline 2-Ehtylfuran & $0.80 \pm 0.33 \mathrm{a}$ & $6.80 \pm 1.4 *$ & $6.56 \pm 3.24 \mathrm{~b}$ & $7.22 \pm 0.20$ \\
\hline Hexanal & $0.74 \pm 0.64$ & $8.64 \pm 7.51 *, \mathrm{a}$ & $0.36 \pm 0.59$ & $0.04 \pm 0.01 \mathrm{~b}$ \\
\hline (E)-2-hexanal & $0.02 \pm 0.03$ & $26.11 \pm 4.24 *, \mathrm{a}$ & $0.01 \pm 0.01$ & $0.00 \pm 0.00 \mathrm{~b}$ \\
\hline Nonanal & $0.17 \pm 0.03 \mathrm{a}$ & $0.39 \pm 0.07$ & $1.2 \pm 0.33 b$ & $0.70 \pm 0.60$ \\
\hline $\begin{array}{l}\text { Total fatty acid- } \\
\text { derived volatiles }\end{array}$ & $0.75 \pm 0.65$ & $34.75 \pm 11.5 *, \mathrm{a}$ & $0.37 \pm 0.60$ & $0.04 \pm 0.01 b$ \\
\hline
\end{tabular}

${ }^{\mathrm{z}}$ Calculation of percentage $=$ peak intensity of individual volatile or category volatile (sulfides and fatty acid-derived volatile) divided by total peak intensity.

* Asterisk indicates significant difference before and after steaming within the same treatment group by Student's T-test $(P \leq 0.05)$., $n=3)$.

Different letters indicate significant difference with or without MeJA treatment within the same cooking treatment group by Student's T-test $(P \leq 0.05, n=3)$ 
Table 4.3 Sensory attribute evaluation of raw and steamed broccoli with or without $250 \mu \mathrm{M}$ methyl jasmonate treatment.

\begin{tabular}{lcccc}
\hline & \multicolumn{2}{c}{ Raw broccoli } & \multicolumn{2}{c}{ Steamed broccoli } \\
\cline { 2 - 5 } & Control & MeJA-treated & Control & MeJA-treated \\
\hline Appearance and color & $5.9 \pm 0.83$ & $5.8 \pm 0.94$ & $6.0 \pm 0.88$ & $6.2 \pm 0.83$ \\
Taste and mouthfeel & $5.7 \pm 1.30$ & $4.9 \pm 1.27^{*}$ & $5.7 \pm 1.19$ & $5.4 \pm 1.09$ \\
Texture and mouthfeel & $5.8 \pm 1.22$ & $5.2 \pm 1.43^{*}$ & $5.9 \pm 0.99$ & $5.7 \pm 0.96$ \\
Overall liking & $5.7 \pm 1.18$ & $5.1 \pm 1.19^{*}$ & $5.8 \pm 1.05$ & $5.7 \pm 0.95$ \\
\hline
\end{tabular}

Data expressed as mean value \pm standard deviation $(N=58)$. Attributes were scored using a 7point hedonic scale where $1=$ dislike extremely, $2=$ dislike very much, $3=$ dislike moderately, $4=$ neither like nor dislike, $5=$ like moderately, $6=$ like very much, and $7=$ like extremely.

*Asterisk indicates significant difference by Student's T-test $(P \leq 0.05)$. 
Table 4.4. Metabolites that high in variable influence on projection (VIP) by partial least square - regression model using overall liking score and taste/flavor score.

\begin{tabular}{lclc}
\hline From model using overall liking score & \multicolumn{2}{l}{ From model using taste \& flavor score } \\
\hline Metabolites & VIP & Metabolites & VIP \\
\hline NMI3ACN $^{z}$ & 1.88 & 4-Methoxyglucobrassicin & 1.73 \\
NMI3C & 1.87 & Neoglucobrassicin & 1.71 \\
(E)-2-hexanal & 1.86 & 2-Ehtylfuran & 1.70 \\
Brassicianapin & 1.84 & (E)-2-hexanal & 1.68 \\
NMI3CA $^{\mathrm{x}}$ & 1.79 & NMI3ACN & 1.66 \\
4-Methoxy-glucobrassicin & 1.63 & NMI3C & 1.65 \\
2-Ehtylfuran & 1.63 & Glucoerucin & 1.57 \\
Glucoerucin & 1.56 & Brassicanapin & 1.54 \\
Indole-3-carbinol & 1.53 & NMI3CA & 1.50
\end{tabular}

${ }^{\mathrm{z}} \mathrm{NMI3ACN}=N$-methoxyindole-3-acetonitrile; ${ }^{\mathrm{y}} \mathrm{NMI} 3 \mathrm{C}=N$-methoxyindole-3-carbinol; ${ }^{\mathrm{x}}$ NMI3CA $=N$-methoxyindole-3-carboxyaldehyde 
(A)

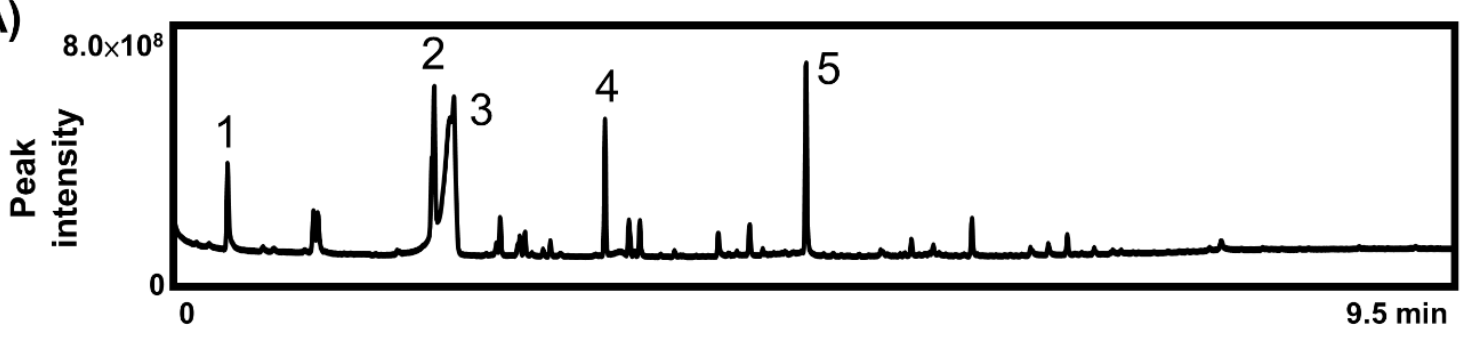

(B)

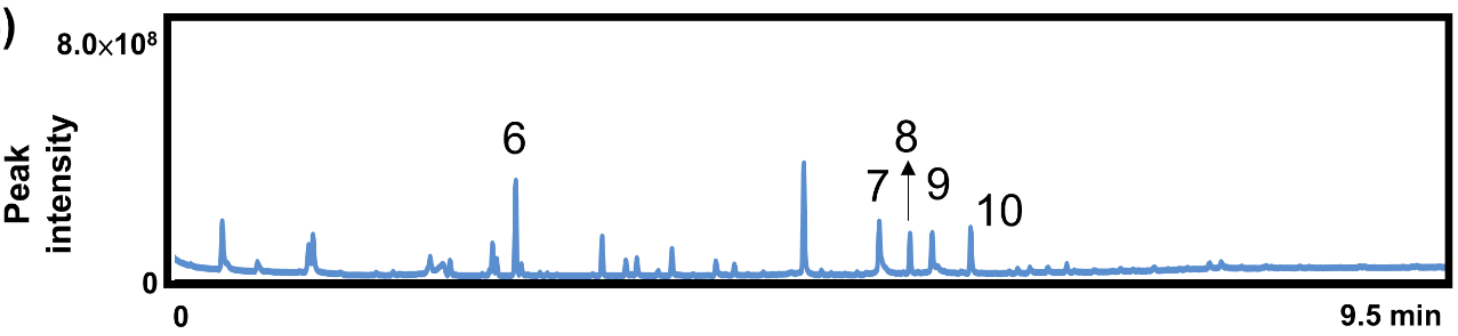

(C)

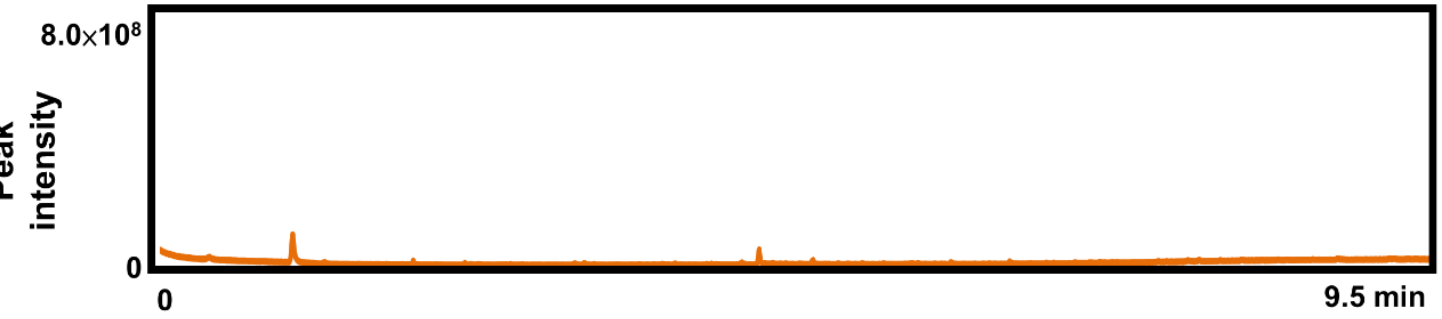

(D)

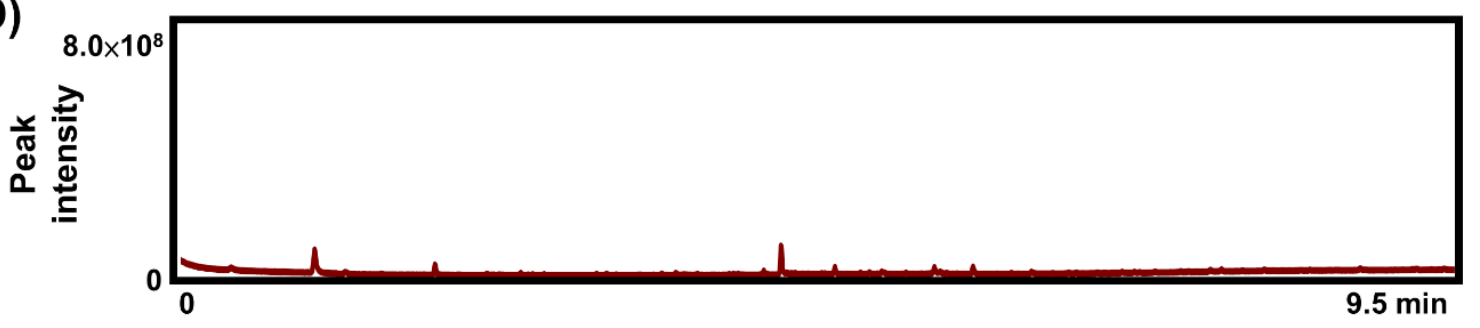

Figure 4.1 GC chromatograph of glucosinolate hydrolysis products from control and MeJAtreated broccoli. (A) raw control broccoli, (B) raw MeJA-treated broccoli, (C) Steamed control broccoli, and (D) Steamed MeJA-treated broccoli.

The major hydrolysis products in the graph: (1) 3-pentenenitrile, (2) 3-butenyl isothiocyanate, (3) 1-cyano-2,3-epithiopropane, (4) erucin nitrile, (5) sulforaphane nitrile, (6) 1-cyano-3,4epithiobutane, (7) $\mathrm{N}$-methoxy-indole-3-carbinol, (8) $\mathrm{N}$-methoxy-indole-3-carboxaldehyde, (9) $\mathrm{N}$ methoxy-indole-3-acetonitrile, and (10) indole-3-acetonitrile. 
(A) Amino acids
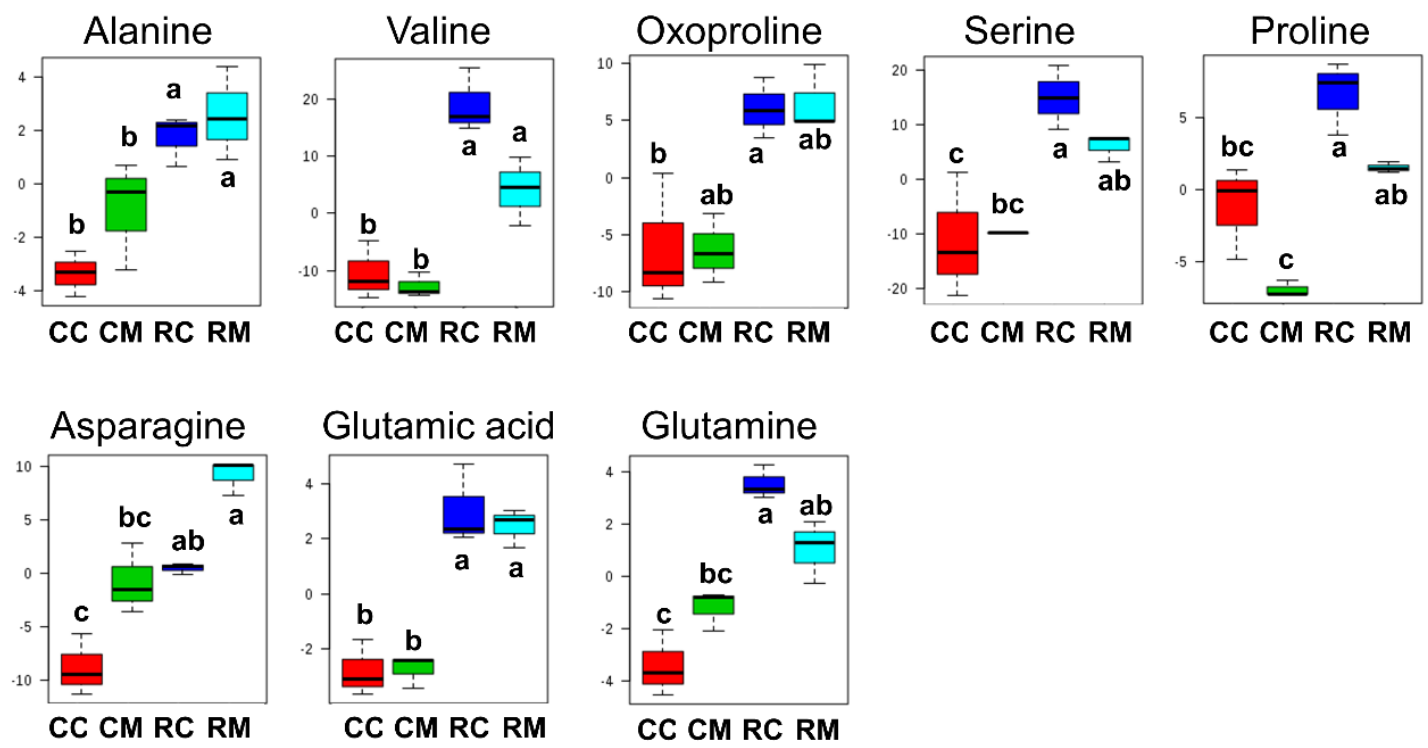

(B) Organic acids
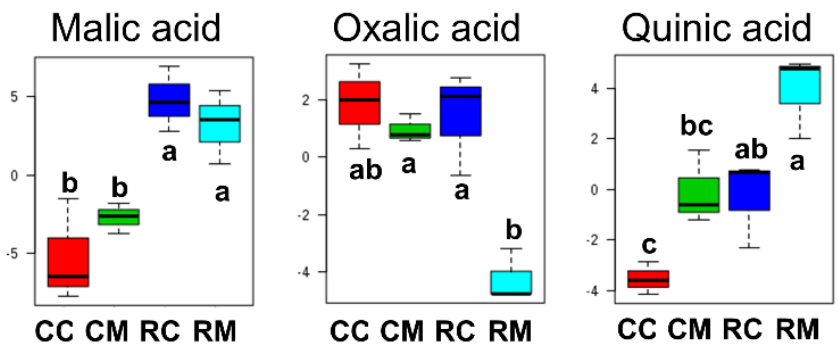

(C) Sugars and derivatives
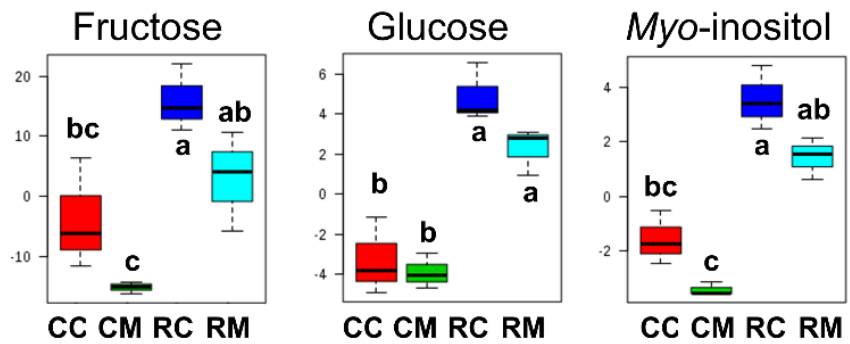

Figure 4.2. Significant primary metabolites (A) amino acids, (B) organic acids, and (C) sugars and derivatives of raw and steamed 'Green Magic' broccoli with or without $250 \mu \mathrm{M} \mathrm{MeJA}$ treatment.

X-axis: sample groups (CC: steamed control; CM: steamed MeJA; RC: raw control; RM: raw MeJA). Y-axis: peak intensity after normalizing to internal standard. Different letters indicate significant difference between different groups by Tukey's test at $P \leq 0.05$. 


\section{Chapter 5 Evaluating the feasibility of exogenous methyl jasmonate application on Brassica vegetables using consumer survey and producer focus group}

\subsection{Abstract}

Application of methyl jasmonate (MeJA) enhances the production of the potential healthpromoting glucosinolates (GS). Neoglucobrassicin, an indolyl GS, in broccoli is especially enhanced because it mimics insect attacks. MeJA application also increases the concentration of neoglucobrassicin-derived hydrolysis products, which are anti-carcinogenic. However, if consumers are unwilling to pay more for MeJA-treated broccoli or if farmers are unwilling to adopt the practices owing to the lack of increased gross income, then the application of MeJA would be limited to the small-scale experimental setting. The aim of this study was to (1) identify potential consumers who are willing to pay more for the MeJA-treated broccoli by a designed consumer survey, (2) collect farmers' feedback on MeJA application by a focus group discussion, and (3) determine if MeJA treatment can be economically feasible in the small-scale farm by incorporating the cost of farm business and the concept of elasticity.

Results of the consumer survey $(n=48)$ showed that the consumers who considered "anticancer broccoli" as the most attractive trait were also willing to pay higher price $(\$ 1.00$ or $\$ 1.50$ per head more) when compared to those who were willing to pay $\$ 0.25, \$ 0.50$, and $\$ 0.75$ more per head. This implies that the potential health-promoting effect of MeJA-treated broccoli may be able to increase the sales price. However, the feedback from the local farmers of the focus group showed that the significant bitterness in raw MeJA-treated broccoli may deter consumers from buying MeJA-treated broccoli. Moreover, the sale quantity of broccoli will affect the gross income of a farm. The scenario with the potential MeJA application cost and the 
sales quantity of broccoli from a small farm showed that MeJA-treated broccoli may not bring higher gross income to a small-scale family-owned farm.

Collectively, the feasibility of MeJA treatment on broccoli in a small-scale direct marketing farm is limited. Because the farmers directly face their customers, the taste of broccoli is the biggest concern for farmers selling MeJA-treated broccoli. However, this finding only showed the limitations of MeJA application to direct-marketing farm, such as growers in farmers market in this study. The results from the consumer survey showed the high potential of MeJAtreated broccoli if consumers are well-educated and well-informed of the high nutrition value of MeJA-treated broccoli. Therefore, the feasibility of MeJA treatment may be higher in different types of markets. 


\subsection{Introduction}

Methyl jasmonate (MeJA) is a phytohormone that plays an important role in plant insect herbivory (Wasternack \& Hause, 2013). One of the defense mechanisms of MeJA in plants is to activate defense compounds, usually plant secondary metabolites that combat the insect herbivory. Endogenous MeJA also serves as a signal molecule that activates the defense system in the distant tissues or neighboring plants. Owing to its role in the inducible plant defense system, exogenous MeJA application is considered a method to experimentally mimic insect herbivory at the biochemical and molecular levels (Benevenuto et al., 2019; Chiu et al., 2018; Seldal, Hegland, Rydgren, Rodriguez-Saona, \& Töpper, 2017).

The application of MeJA increases health-promoting compounds, the glucosinolates (GS), in Brassica vegetables, such as broccoli (Ku et al., 2014). Among all of the GS, two indolyl GS, glucobrassicin and neoglucobrassicin, and an aromatic GS, gluconasturtiin, were reported to be increased by MeJA application (Ku et al., 2014). Moreover, the health-promoting properties (anti-inflammatory or chemopreventive) do not come from GS themselves; instead, hydrolysis products of GS are responsible for the bioactivity (Becker \& Juvik, 2016).

Despite the positive effect of GS and its hydrolysis products for humans, consumers might need to pay a higher price for MeJA-treated broccoli because of the application cost of MeJA. Identifying the types of consumers who are willing to pay more for the "value-added" broccoli, even at a premium price, is critical. Additionally, understanding how elasticity, an indicator of how sales quantity will fluctuate due to the price, will change the sale quantity of MeJA-treated broccoli is crucial. A 2010 study revealed that the estimated absolute value of mean price elasticity of vegetables is -0.58 (Andreyeva, Long, \& Brownell, 2010), which is based on primary demand (commodity or category demand) and is lower than the price elasticity of soft 
drinks (elasticity $=-0.79$ ). In 2012, USDA provided the estimated demand elasticity, -0.79 , of vegetables (excluding lettuce and tomato) (Okrent \& Alston, 2012). Despite the difference in estimate, vegetable is considered to be inelastic: the decreased percentage of sale quantity is below the increased percentage of new price.

Although MeJA application has been investigated extensively, it is primarily used in smallscale experiments. In this study, the feasibility of MeJA on broccoli production was evaluated, with particular attention paid to the farmers' experience. This step is crucial before MeJA application can be applied to real-world practices or large-scale production. Furthermore, we need to identify the constraints and perceptions of applying MeJA from local farmers along with the marketing challenges they face. Focus groups (or focus group discussions) allow researchers to collect qualitative data from the participants and interact with the participants about a certain topic. These such groups have been used as a tool to identify people's preferences and thoughts toward a product or a service, as well as collect quantitative results in an economic feasibility assessment (Krueger, 2014). Additionally, focus groups have been utilized to access the needs of the farmers (Bailey, Arnold, \& Igo, 2014) and address the obstacles in the agriculture business (Mwaijande, Miller, Wailes, \& Peterson Jr., 2009). Therefore, it can be used to analyze a wide range of topics from a variety of individuals, and it can provide valuable feedback from the farmers.

In the food supply chain, an agricultural product is harvested from the supplier (farmers), processed, and transported to the retailers (postharvest process); afterwards, it is sold to the consumers. Research about the exogenous application of MeJA in Brassica vegetables has been conducted on the postharvest process or the application itself; however, to our knowledge, feedback on the exogenous MeJA application from consumers and farmers is lacking. The aim of 
this study was to evaluate the feasibility of MeJA application by combining consumer surveys and farmer feedback. The ordered probit regression model was used to analyze the existing consumer survey data to identify the types of consumers who are willing to purchase MeJAtreated broccoli. Focus group was formed with the growers in the greater Morgantown area to collect their perspectives on MeJA application in the real-world. Lastly, a scenario-based analysis was developed to evaluate the feasibility of MeJA treatment in a small-scale family farm. 


\subsection{Methods}

\subsubsection{The consumer survey}

To investigate how broccoli was incorporated into the daily diet and the broccoli consumption habitat, the 60 panelists recruited in Chapter 3 were given a questionnaire (Supplementary Document 2) before the sensory evaluation. Panelists were recruited during the summer of 2017 by flyers in the building and in-person invitations at Davis College of West Virginia University; recruitment was not limited to Davis College colleagues. All panelists participated voluntarily, and no incentives were provided. All responses were recorded in a printed questionnaire anonymously during the session; however, all responses were typed in a Google Form for preliminary data visualization.

\subsubsection{The consumer survey: data Analysis}

To understand the type of consumer willing to pay more for broccoli, an ordered probit model was conducted with Stata (version 13, College Station, Texas, 77845) using the data from those who were willing to pay more for broccoli that has twice the amount of cancer fighting compounds $(\mathrm{n}=48)$. The dependent variable was the premium price that a customer was willing to pay ( $\$ 0.25, \$ 0.50, \$ 0.75, \$ 1.00$, and $\$ 1.50$ more per broccoli head), and the independent variables were: (1) age (between 18 and 35 years old, between 36-55 years old, or 56 years old and above), (2) purchasing frequency (purchase broccoli at least weekly or monthly), (3) expected nutritional value (vitamins and minerals, antioxidant compounds, fibers, and others), and (4) ideal broccoli traits (anticancer, better tasting , and longer shelf-life broccoli). Five marginal effects were evaluated with each selected premium price $(\$ 0.25, \$ 0.50, \$ 0.75, \$ 1.00$, and $\$ 1.50$ more per broccoli head). 


$$
y_{i}^{*}=\beta_{0}+\beta_{1} \text { Age }_{i}+\beta_{2} \text { Frequency }_{i}+\beta_{3} \text { Nutrition }_{i}+\beta_{4} \text { Attraction }_{i}+\epsilon_{i},
$$

where:

$$
\begin{aligned}
& y_{i}^{*}=\text { the unobserved premium price consumers are truly willing to pay, } \\
& y_{i}=0 \text { if } 0 \leq y^{*} \leq 0.25, \text { indicating the participant chose a premium price of } \$ 0.25, \\
& y_{i}=1 \text { if } 0.26 \leq y^{*} \leq 0.50 \text {, indicating the participant chose a premium price of } \$ 0.50, \\
& y_{i}=2 \text { if } 0.51 \leq y^{*} \leq 0.75, \text { indicating the participant chose a premium price of } \$ 0.75, \\
& y_{i}=3 \text { if } 0.76 \leq y^{*} \leq 0.10, \text { indicating the participant chose a premium price of } \$ 1.00, \\
& y_{i}=4 \text { if } 0.11 \leq y^{*}, \text { indicating the participant chose a premium price of } \$ 1.50, \\
& \beta=\text { the estimated vector of each parameter, } \\
& \epsilon_{i}=\text { error term, which is normally distributed with a mean of zero and standard deviation of }
\end{aligned}
$$
one.

$A g e_{i}$ represents the ages of the participants. When $\mathrm{i}=1$, the participant was between the ages of 18 and 35; when $i=2$, the participant was between the ages of 36 and 55; when $i=3$, the participant was 56 or older.

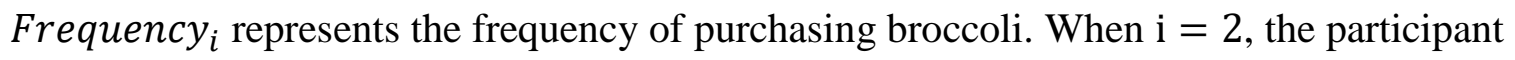
purchased broccoli at least weekly (including both daily and weekly); when $\mathrm{i}=3$, the participant purchased broccoli monthly.

Nutrition $_{i}$ represents the main nutritional component that the participants were expecting to gain when they eat broccoli. When $\mathrm{i}=1$, the participant expects vitamins and minerals; when 
$\mathrm{i}=2$, the participant expects antioxidant compounds; when $\mathrm{i}=3$, the participant expects fibers; when $\mathrm{i}=0$, the participant expects another benefit.

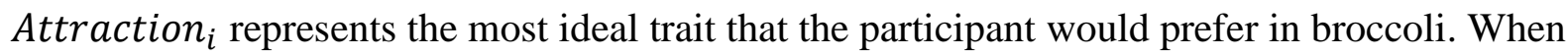
$\mathrm{i}=1$, the participant preferred an anticancer benefit; when $\mathrm{i}=0$, the participant would prefer another benefit (better tasting broccoli and longer shelf-life).

\subsubsection{Farmer Focus Group}

A farmers' focus group was formed to investigate their perception on the practicability of MeJA application. The recruitment of focus group participants was based on the pre-existing list of local growers provided by the Small Farm Center at West Virginia University. We particularly recruited farmers on the list who currently grow or have previously grown broccoli and five farmers were recruited for the focus group discussion.

The farmer focus group session lasted an hour and a half. All participants were given a questionnaire to fill in demographics and questions related to the broccoli cultivation in their farms (Supplementary Document 3). A 10 min presentation was given at the beginning of the session to introduce the effect of MeJA application on broccoli and other Brassica vegetables, and to show the approximate cost of MeJA application. The focus group participants were asked for their opinions on the following two themes:

1. What do you think about applying MeJA to the production of broccoli?

2. What are the major challenges you face in broccoli or Brassica vegetables production?

Each theme included three to five questions for the focus group to discuss, and the questions were listed in the questionnaire that participants received when they arrived at the meeting. The questionnaire and the recruiting process were approved by the West Virginia 
University Institutional Review Board for the Protection of Human Subjects in Research (IRB)

(Supplementary Document 4). Each participant read and signed the documents, which included a video recording consent form, purpose of the study, participant anonymity, and the use and handling of the collected qualitative information (Supplementary Document 5).

\subsubsection{Farmer Focus Group: Data Analysis}

Electronic transcript and audio recording were conducted during the focus group session, and the transcript was carefully analyzed by the qualitative online platform ATLAS.ti ${ }^{\circledR}$ CLOUD (ATLAS.ti Scientific Software Development GmbH, Liezenburger, Berlin, Germany). The analysis involved thematic coding related with the two above-mentioned themes. Four codes were chosen to extract the farmers' feedback from the transcript, which included advantages, disadvantages, challenges, and new thoughts toward to the MeJA application. Using the online platform ATLAS.ti@ CLOUD, the core content of each participant was identified in each code, and therefore, the trend could be seen after the content analysis.

\subsubsection{The Consumer Survey Demographics}

We received 60 questionnaires from consumers, and the ratio of female to male was 6:4 among all participants. In the consumer survey, $73 \%$ of the panelists were between 18-35 years old; $10 \%$ of the panelists were between $36-54$ years old, and the rest of the panelists (17\%) were older than 55 .

\subsubsection{Farmer's Focus Group Demographics}

We recruited five local farmers for the focus group discussion. Four of them were from West Virginia, and one of them was from Maryland. They all sold their fresh products in Morgantown Farmers' Market. The focus group included two male and three female participants. 
Three of them own a conventional farm, and one of them owns an organic farm. One farmer did not identify the operation style, but the farm website states that the farm is "low input and nonGMO." Four growers were above 30 years old, and one farmer was less than 30 years old. The farm size ranged from 1 to 315 acres.

\subsection{Results and discussions}

\subsubsection{The Consumer Survey}

Based on the questionnaire, all panelists enjoyed eating fruits and vegetables. However, we might have encountered a sampling bias, because all participants were voluntary (Salkind, 2010). Ninety-five percent of the participants stated that they liked broccoli. When asked about why they enjoyed broccoli, the most prominent reason was (1) nutritional value (48\%) and (2) flavor (43\%). Vitamins, minerals, and fiber were selected as the three main nutrients that panelists expected to gain from consuming broccoli. These results agree with a previous study, where taste and health benefits were the two main reasons for vegetable consumption (Schätzer, Rust, \& Elmadfa, 2010). When asked which additional broccoli traits would be most attractive to panelists, $45 \%$ stated that better tasting broccoli would be the most attractive trait, followed by anti-cancer (30\%) and longer shelf-life broccoli (19\%). Along with the response to the previous question (why do you purchase broccoli), taste of broccoli is the most critical factor to drive the purchase and consumption, which corresponds well with a previous study (Schätzer et al., 2010).

Based on the responses, $88 \%$ of the panelists purchased fresh broccoli, and $65 \%$ of the panelists purchased fresh broccoli on a regular basis. For the panelist who purchased fresh broccoli, the most important factor to them was the visual color (greenness). This result agreed with previous research that appearance was an important factor in the purchase of vegetables 
regardless of household income (Chikkamath, Atteri, Srivastava, \& Roy, 2012). Meanwhile, half of the panelists $(52 \%)$ consumed broccoli (either fresh or frozen) weekly, and another $43 \%$ of the panelists consumed broccoli monthly.

Using the questionnaire, we also investigated how the panelists consumed broccoli, specifically whether they ate raw or cooked broccoli, because cooking affects the sensory evaluation (Chapter 3) and nutritional quality (Chapter 4) of broccoli. From the responses, more than half $(52 \%)$ of the panelists steamed the broccoli before consumption, followed by boiling (14\%). Of the proposed methods, only $8.5 \%$ of the panelists ate raw broccoli.

Because we wanted to know if MeJA-treated broccoli would have a better market price, we asked the panelists about the potential increase of broccoli sales price. Eighty-one percent of the panelists $(n=48)$ were willing to pay more for broccoli with twice the amount of cancer fighting compounds (Figure 5.1).

To further elaborate on this result, we produced an ordered probit model to investigate the types of consumers who are willing to pay more. Consequently, we found that consumers who considered anticancer benefits of broccoli as an important trait (Question 12 in the questionnaire) would be willing to pay a higher price ( $\$ 1.00$ or $\$ 1.50$ more per head) at $P \leq 0.05$ (Table 5.1).

\subsubsection{Producer focus group-adapting MeJA application in the current practice}

The potential price increase induced by MeJA application in broccoli cultivation was calculated using a $250 \mu \mathrm{M}$ MeJA solution (mixing 57.81 $\mu \mathrm{L}$ MeJA per L of water) (Ku et al., 2014b) as the optimum spray regime, and each plant was sprayed with a $0.1 \mathrm{~L}$ solution. The equation below was used to calculate the cost of spraying: 


$$
\begin{aligned}
\text { increased price per head } & =\frac{\text { Price of MeJA per bottle }}{\text { Number of broccoli sprayed per bottle }}, \\
\text { increased price per head } & =\frac{\$ 125}{25 m L * 10^{3}(\mu L) \div 57.81 \mu L \div 0.1 L}, \\
& =\$ 0.03
\end{aligned}
$$

Where:

- Price of MeJA per bottle as obtained from the Sigma website (St. Louis, MO, USA) and MeJA can be purchased as $25-\mathrm{mL}$ or 5-mL glass bottle. No shelf life was suggested, nor was the expiration date suggested in the safe data sheet;

- Number of broccoli sprayed was determined by how many broccoli can be sprayed with a bottle of $25-\mathrm{mL}$ MeJA.

If using a 5-mL bottle MeJA under the same condition, the application cost of is $\$ 0.04$ derived from the same equation.

We conducted the focus group discussion from local growers to collect feedback about the advantages or disadvantages of exogenous MeJA application in the current broccoli or Brassica vegetables production. Although we have calculated the potential cost of MeJA application, we did not include other potential cost, such as labor or storage, because we did not have such data. After the discussion, the focus group came to the conclusion that the cost of exogenous MeJA application would be minimal if it was combined with another application. One grower also mentioned that there was no apparent difficulty in the current proposed application method. Aside from the additional cost or difficulties, two growers specifically asked about the 
timing of MeJA application during the harvest period and whether rain washes off the effects of the treatment.

Insects, high humidity, and heat are common challenges of broccoli cultivation in Morgantown, West Virginia, and the western Maryland area according to the focus group. All participants in the focus group commented about the insect issue, and humidity and heat were mentioned only two times. Other challenges, such as the uniformity of broccoli head, were also mentioned. High heat usually reduces the uniformity of broccoli head (T. Björkman \& Pearson, 1998); therefore, this uniformity issue was expected to be mentioned.

The major issue mentioned by the local producers was the taste of broccoli. Throughout the discussion sessions, the word "taste" or "bitterness" was mentioned up to six times from different participants. Focus group participants sell their fresh products at the farmers' market, wholesale through a growers' cooperative, to a school's community supported agriculture (CSA) program, and on a Facebook group. In the farmers' market, broccoli is usually sold at a higher price compared to wholesale. One trend identified from the discussion was that majority of the participants faced their customers directly. Participants considered the taste of broccoli as the most important quality. If the taste did not meet their expectations, the farmers would lose their customers. For example, one participant mentioned “.... if kids don't eat it they wouldn't buy it anymore..." Therefore, the biggest concern of MeJA treatment on broccoli for growers would be the bitterness.

One discussion in the focus group was about how consumers eat broccoli. Focus group participants mentioned that some consumers prefer to eat broccoli raw. One participant stated that "...lots of them (the customers) eat it (broccoli) raw." Therefore, the detectable bitterness in raw MeJA-treated broccoli (see Chapter 3) would make exogenous MeJA application an 
improper treatment to the focus group participants, because the taste is directly associated with their sales. However, this feedback conflicted with the consumer survey we conducted (see section 4.2) where above $50 \%$ of the consumers stated that they cooked broccoli. One grower stated “...I think people at the farmers' market are more concerned about the taste...”. Another grower also mentioned that some customers purchased broccoli from his farm because of the raw broccoli taste. In the consumer survey, the most important reason to make a broccoli purchase was its nutritional benefits, which was weighed at $48.3 \%(5.2 \%$ higher than the flavor of the broccoli).

Combing all the information, the focus group emphasized the importance of the marketing strategy of MeJA-treated broccoli. Although they shared the concerns about the taste of broccoli, two growers also pointed out the possibility of accepting MeJA-treated broccoli if its increased health-benefit can be properly advertised. The farmers were asked which type of evidence they would need to convince them to adopt MeJA treatment. All local growers suggested that marketing campaigns and a new name associated with the high anti-cancer activity of MeJA-treated broccoli. One grower also suggested that MeJA treatment might be acceptable for consumers in the CSA program. Because farmers communicate with consumers before they receive the products from CSA, the farmers have an opportunity to explain the benefit of the application and how it potentially increases the bitterness in broccoli.

To further evaluate the feasibility of the exogenous application of MeJA, the focus group was asked if there were other alternative crops that might be suitable for this application. They suggested using MeJA treatment on Brassica vegetables that are already bitter. For example, kale or swiss chard. Interestingly, collards were mentioned by a grower and he mentioned "Collards are not that bitter. Maybe we can sell more collards that way," which implied that the 
increased bitterness in collards may be more flavorful to the grower and the consumer. In addition to the leafy Brassica vegetables, cauliflower was mentioned by three growers. According to the farmers, cauliflower was very popular, especially sold to school food services.

The farmers focus group provided perceptions of MeJA application from five local growers in the Morgantown and western Maryland area. The biggest concern of the growers was the bitterness caused by the MeJA treatment; however, there was no significant increase in the cost of labor associated with the proposed spraying routine. Local growers also suggested that a marketing strategy be developed to increase the possibility of purchase. Some alternative vegetables (kale, swiss chard, collard, and cauliflower) were suggested for MeJA application. The information provided by the focus group will be compared to the results from the consumer survey in section 4.2, and further discussion will be stressed in Chapter 6 .

\subsubsection{Cost-Benefit Analysis}

We calculated the material cost of MeJA application and constructed a proposed scenario using the data provided by one grower who attended the focus group (Table 5.2). The assumptions for this scenario were based upon the cost of broccoli cultivation (seeds, fertilizer, and labor), optimization of MeJA application, and focus group discussion.

\section{$\underline{\text { Scenario assumptions }}$}

- Elasticity is set at -0.58 (relatively inelastic) and -0.79 (relatively elastic) according to previous literature (Andreyeva, Long, \& Brownell, 2010; Okrent \& Alston, 2012)

- Farm size of focus group participants are small (<279 acres) (Hoppe, 2014).

- $5 \mathrm{~mL}$ of MeJA purchased from Sigma (St. Louis, MO, USA) costs $\$ 37.9$, and $25 \mathrm{~mL}$ MeJA from the same company costs $\$ 125$. Each broccoli plant will need $300 \mathrm{~mL}$ of spray 
to be effective according to the literate (Ku et al., 2014b). No shelf-life or expiration date was suggested by the safe data sheet.

- If using $5 \mathrm{~mL}$ bottle of MeJA using the optimized spray regime (a $250 \mu \mathrm{M}$ MeJA application), the application cost of a broccoli plant is $\$ 0.04$. If using $25 \mathrm{~mL}$ bottle of MeJA under the same condition, the application cost of a broccoli plant is $\$ 0.03$.

In a 2012 USDA study (Okrent \& Alston, 2012), "healthy" products, such as vegetables and fruits, were shown to be less sensitive to price changes when compared to "unhealthy" products (e.g., cake and cookies). According to the proposed scenario, exogenous MeJA application may increase gross income by $2.58 \%$ to $14.13 \%$ when elasticity is set at -0.58 , or increase by $1.25 \%-7 \%$ when elasticity is set at -0.79 for small-scale farm. In this proposed scenario, the gross income of the farmers was always lower than that based on broccoli without MeJA treatment ven with lower sales quantity (50 broccoli). The simulated calculation indicated that exogenous MeJA treatment may bring extra revenue for small farm with small-scale of broccoli production if growers are willing to incorporate MeJA application into their routine practice.

Elasticity can change with income level: for instance, lower-income households are more sensitive to the price changes (Ni Mhurchu et al., 2013). Therefore, depending on the target demography, the elasticity can increase or decrease. The limitation of this study is mainly attributed to the recruited panelists and local producers. All recruited panelists in the consumer survey are associated with Davis college or WVU, and therefore the diversity of occupations and education level is lacking. As the recruiting time occurred at the summer vacation, we could not recruit many college students, and thus age and income level of panelists was relatively homogenous. Sensitivity of bitterness changes with age, and children are usually highly sensitive 
to the bitterness (Cowart, Yokomukai, \& Beauchamp, 1994; Mennella \& Bobowski, 2015). Therefore, it is important to consider the age of recruited panelists in this study.

Socioeconomic status can largely affect the food choice when individual is making food purchasing decision (Turrell, Hewitt, Patterson, Oldenburg, \& Gould, 2002), and the food purchasing decision from socioeconomically-disadvantaged groups are less likely to meet dietary guideline recommendations (Giskes, Van Lenthe, Brug, Mackenbach, \& Turrell, 2007). Therefore, the results from this study can only apply to certain demographic population. WVU locates at Morgantown, West Virginia and is experiencing economic growth in terms of increasing job numbers (WV News, 2019); however, West Virginia, as a whole, is economically disadvantaged and has lowest personal income in the US (USDC, 2019). As the survey was conducted at WVU, the applicability of the result may only apply to the demographic population with similar demography. On the other hand, survey results (diet habit, food choice, and willingness-to-pay for premium price broccoli) may change when the recruited panelists are from high-income or metropolitan area.

\subsection{Conclusion}

Recent research on MeJA application in different crops has provided the evidence on how the application of MeJA can increase the produce quality. However, the feasibility of MeJA application should be evaluated in the scale of real production. The findings in this study show that MeJA-treated broccoli may have a niche in the market for consumers' who are willing to pay more for broccoli with better nutritional value and small-scale broccoli grower may be benefit from this application as well. However, local growers are concerned about the significant 
bitterness in raw MeJA-treated broccoli. If MeJA-treated broccoli was sold through direct marketing by farmers, an education program or a marketing campaign would be needed so that consumers understand the nutritional value of MeJA-treated broccoli and the proper way to cook it to achieve a better taste. Lastly, MeJA-treated broccoli may be suitable for CSA consumers, because famers have the opportunity to explain the benefit to the educational program themselves. 
Table 5.1 Marginal effect of the ordered probit model for the extra premium price that consumers are willing to pay.

\begin{tabular}{cccccc}
\hline & $\begin{array}{c}\text { Marginal } \\
\text { effect for }\end{array}$ & $\begin{array}{c}\text { Marginal } \\
\text { effect for }\end{array}$ & $\begin{array}{c}\text { Marginal } \\
\text { effect for }\end{array}$ & $\begin{array}{c}\text { Marginal } \\
\text { effect for }\end{array}$ & $\begin{array}{c}\text { Marginal } \\
\text { effect for }\end{array}$ \\
& $\$ 0.25$ extra & $\$ 0.50$ extra & $\$ 0.75$ extra & $\$ 1.00$ extra & $\$ 1.5$ extra \\
\hline Age & 0.008 & 0.007 & 0.003 & -0.010 & -0.009 \\
Frequency & -0.027 & -0.024 & -0.013 & 0.033 & 0.031 \\
Nutrition & -0.002 & -0.001 & -0.000 & 0.002 & 0.001 \\
Attraction & $-0.118^{*}$ & $-0.120^{*}$ & -0.081 & $0.124^{*}$ & $0.194^{*}$ \\
\hline
\end{tabular}

* indicates the significant difference by $p$-value $\leq 0.05$. 
Table 5.2 Simulation of consumer demand using estimated elasticity

\begin{tabular}{|c|c|c|c|c|c|c|}
\hline \multicolumn{7}{|c|}{ Elasticity $=-0.58^{z}$} \\
\hline $\begin{array}{c}\text { Increased } \\
\text { priced per head }\end{array}$ & $\begin{array}{c}\text { Sale price } \\
\text { per head }\end{array}$ & $\begin{array}{c}\text { Sale } \\
\text { quantity }\end{array}$ & Revenue & $\begin{array}{l}\text { Revenue } \\
\text { increase }\end{array}$ & $\begin{array}{l}\text { Gross income } \\
(5 \mathrm{~mL} \text { MeJA) }\end{array}$ & $\begin{array}{l}\text { Gross income } \\
(25 \mathrm{~mL} \\
\text { MeJA })\end{array}$ \\
\hline 0 & 4 & 50 & $\$ 200$ & $0 \%$ & $\$ 143.43$ & $\$ 149.13$ \\
\hline 0.25 & 4.25 & 48.27 & $\$ 205.15$ & $2.58 \%$ & $\$ 148.57$ & $\$ 154.28$ \\
\hline 0.5 & 4.5 & 46.70 & $\$ 210.15$ & $5.08 \%$ & $\$ 153.58$ & $\$ 159.28$ \\
\hline 0.75 & 4.75 & 45.26 & $\$ 214.99$ & $7.50 \%$ & $\$ 158.41$ & $\$ 164.12$ \\
\hline 1 & 5 & 43.95 & $\$ 219.75$ & $9.86 \%$ & $\$ 163.18$ & $\$ 168.88$ \\
\hline 1.5 & 5.5 & 41.61 & $\$ 228.86$ & $14.43 \%$ & $\$ 172.28$ & $\$ 177.99$ \\
\hline \multicolumn{7}{|c|}{ Elasticity $=-0.79^{y}$} \\
\hline $\begin{array}{c}\text { Increased } \\
\text { priced per head }\end{array}$ & $\begin{array}{l}\text { Sale price } \\
\text { per head }\end{array}$ & $\begin{array}{c}\text { Sale } \\
\text { quantity }\end{array}$ & Revenue & $\begin{array}{l}\text { Revenue } \\
\text { increase }\end{array}$ & $\begin{array}{l}\text { Gross income } \\
(5 \mathrm{~mL} \text { MeJA })\end{array}$ & $\begin{array}{c}\text { Gross income } \\
(25 \mathrm{~mL} \text { MeJA) }\end{array}$ \\
\hline 0 & 4 & 50 & $\$ 200$ & $0 \%$ & $\$ 143.43$ & $\$ 149.13$ \\
\hline 0.25 & 4.25 & 47.66 & $\$ 202.56$ & $1.28 \%$ & $\$ 145.99$ & $\$ 151.69$ \\
\hline 0.5 & 4.5 & 45.56 & $\$ 205.02$ & $2.51 \%$ & $\$ 148.45$ & $\$ 154.15$ \\
\hline 0.75 & 4.75 & 43.66 & $\$ 207.38$ & $3.69 \%$ & $\$ 150.81$ & $\$ 156.51$ \\
\hline 1 & 5 & 41.93 & $\$ 209.65$ & $4.83 \%$ & $\$ 153.08$ & $\$ 158.78$ \\
\hline 1.5 & 5.5 & 38.91 & $\$ 214$ & $7 \%$ & $\$ 157.43$ & $\$ 163.13$ \\
\hline
\end{tabular}

${ }^{\mathrm{z}}$ Data from Andreyeva, Long, \& Brownell, 2010.

${ }^{\mathrm{y}}$ Data from Okrent \& Alston, 2012. 


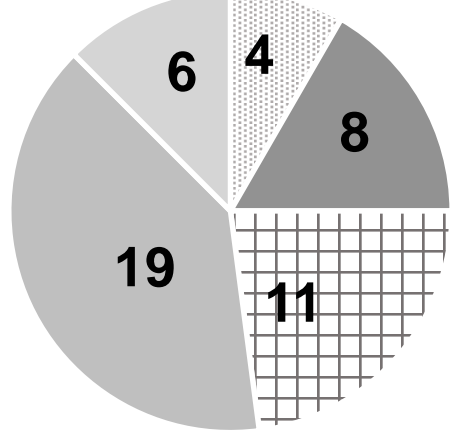

\section{$\$ 0.25$ / head \\ - $\$ 0.50$ / head \\ $\vdash \$ 0.75 /$ head \\ $\$ 1.00 /$ head \\ $\$ 1.50$ / head}

Figure 5.1 Distribution of consumers who were willing to pay more for the broccoli containing twice amount of the anti-cancer compounds $(n=48)$. 


\section{Chapter 6 Summary and discussion with future perspectives}

\subsection{Summary}

The overall goal of this project was to bridge the gap in the research of exogenous MeJA application on Brassica vegetables at the lower end of food supply chain (consumers) (Chapter 3and 4), as well as the further elucidation of the mechanism of exogenous MeJA application and the generalist insect attack on Brassica vegetables (Chapter 1). In addition to this goal, we collected local growers' perspectives on exogenous MeJA application (Chapter 5), because information is lacking on the feasibility of the application from their perspective. The major findings of this project were:

(1) Between exogenous $250 \mu \mathrm{M}$ MeJA application and T. $n i$ treatment, the change in metabolite level and gene expression level was not similar. The variation between replications in the $T . n i$ treatment group was greater than the MeJA application. Exogenous MeJA treatment showed significant effect on gene expression levels, while $T . n i$ treatment did not exhibit the same significance. Changes in GS showed very similar trend - the indolyl GS was increased significantly in both treatments along with the gene expression data from the related genes, i.e., $C Y P 81 F 4, M Y B 34$, and $M Y B 122$. On the other hand, the effect of MeJA and T. $n i$ treatment on primary metabolites were not consistent: MeJA treatment consistently decreased the abundance of primary metabolites, while $T . n i$ treatment did not. Five primary metabolites were selected as biomarkers that are responsible for the separation between control, MeJA treatment, and T. ni treatment.

(2) Steaming for four minutes eliminates the bitter taste by exogenous MeJA application. Raw MeJA-treated broccoli was less acceptable to untrained consumer panelists and 
this result may be stemmed from the high concentration of neoglucobrassicin-derived hydrolysis products in MeJA-treated broccoli.

(3) After 5 min of boiling, MeJA-treated broccoli contained 1.9-fold higher levels of total GS compared to the untreated broccoli. Total GS content of MeJA-treated broccoli in other cooking methods (steaming and microwaving) was not significantly different from the raw MeJA-treated broccoli.

(4) Exogenous MeJA treatment may increase the nutritional quality of cooked broccoli, and MeJA-treated broccoli can be utilized as a value-added ingredient in precooked meals, as the taste of steamed MeJA treated broccoli did not differ from the untreated broccoli.

(5) Bitterness of raw MeJA-treated broccoli may deter farmers from adapting MeJA treatment into their cultivation, as they usually sell their production directly to the consumers and some consumers eat broccoli raw; therefore, exogenous MeJA application may not be feasible for the farms in direct market.

(6) There is a market for nutrition-enhanced broccoli if the broccoli by MeJA application is being advertised properly. Consumers who are concerned about the anti-cancer properties of broccoli were willing to pay extra $\$ 1.00$ or $\$ 1.50$ for broccoli.

(7) Exogenous MeJA application at a small-scale farm may be economically feasible; however, if the absolute value of elasticity of broccoli was larger than 0.80 , the this treatment may be used for the farms growing broccoli for frozen foods or precooked meals, and may be very valuable for the food processing industry. 


\subsection{Discussion}

Scientists have investigated the health-promoting effect of GS, GS hydrolysis products, broccoli extracts, or diet rich in Brassica vegetables. (Zabaras et al., 2018). The consumption with high Brassica vegetables reduces the risk of different illness, including breast cancer (Ambrosone et al., 2004) and cardiovascular disease (Zhang et al., 2011). Health-related and chemopreventive information has been used in the intervention study to examine if the provided information would change the consumers' acceptance of vegetables (Cox, Melo, Zabaras, \& Delahunty, 2012). Among all the Brassica vegetables used in the Cox study, the acceptance of broccoli was not affected by the provided information. Instead, bitterness, flavor, vegetable intake, and gender were factors that affected consumers' liking and acceptance of broccoli. Results from a 2018 study showed a similar result, where health benefit information did not significantly increase the preference of broccoli (Zabaras et al., 2018). Results from the focus group and previous literature identified taste as the factor that will dominate consumer willingness to buy; therefore, flavor and taste-related characteristics should be considered high priority, especially for Brassica vegetables sold directly from farmers to the general public, such as in a farmers' market.

The feasibility of MeJA application is largely dependent on the consumer threshold of tasting bitter compounds, as a result of neoglucobrassicin and the conversion of GS and its hydrolysis products. The increase of neoglucobrassicin and its significant bitter taste in our sensory study only showed the effect of a $250 \mu \mathrm{M}$ MeJA application. According to previous literature (Ku et al., 2014b), the application of as little as $62.5 \mu \mathrm{M}$ of MeJA significantly increase the amount of neoglucobrassicin in broccoli. Most importantly, $62.5 \mu \mathrm{M}$ of MeJA can also successfully induce sulforaphane production, which is a highly bioavailable GS hydrolysis 
product as different MeJA concentration may result in several of signal intensities to affect GS biosynthesis or upstream pathways. If we tentatively use $62.5 \mu \mathrm{M}$ of MeJA application to recalculate the cost using the abovementioned equation, the cost can be reduced to $\$ 0.007$ per broccoli head, which equals to about increase of $\$ 0.028$ per $\mathrm{kg}$ of broccoli, depending on the weight of harvested broccoli. With the minimum cost of MeJA application and the potential price increase, we might achieve a healthier broccoli or other Brassica vegetables without losing the taste.

Whether broccoli is consumed raw or cooked is another consideration in the feasibility of MeJA-treated broccoli. Two-thirds of consumer panelists in our study from the Davis College at West Virginia University often steam or boil broccoli, and only 6 people regularly consume broccoli raw. Different cooking processes result in different levels of GS loss, sulfur volatile formation, and endogenous myrosinase activity (Hanschen et al., 2018). All of the changes will pose an impact on the sensorial quality (Bell et al., 2018). However, from our study (Table 2), we observed that when the broccoli was steamed, there was no significant difference in the preference between control and MeJA-treated broccoli regardless of the GS amount in untreated and MeJA-treated broccoli. Cooking time also plays a role in the perception of bitter taste; for example, Poelman and others reported that children were able to taste more bitterness when broccoli was steamed for long periods of time (12 min) (Poelman, Delahunty, \& de Graaf, 2013). Therefore, how broccoli is processed at home or in the restaurant will affect the feasibility of MeJA application on broccoli. For example, MeJA-treated broccoli may be blanched and sold as frozen broccoli or as an ingredient in precooked meal. Because no detectable differences were found between the steamed control and $250 \mu \mathrm{M}$ MeJA-treated broccoli, broccoli used in the precooked meal can be marketed as a value-added ingredient. 
MeJA application is not only effective on broccoli, but also in other Brassica vegetables. In addition, MeJA treatment may be beneficial with different culinary choices. Increased GS by MeJA has been reported in kale, collard greens, pak choi, and cauliflower (Kim et al., 2017; Kim, Chiu, \& Ku, 2017; Ku et al., 2013). Based on the results in Chapter 3, Chapter 4, and previous studies, exogenous MeJA application will increase the GS amounts in Brassica vegetables and, at the same time, cooked MeJA-treated Brassica vegetables may not taste differently to common consumers. In Southern dishes, collard greens are often cooked in stew and eaten together with stew. In this case, MeJA treatment can increase the concentration of GS in collard greens, and the GS loss in the cooking water (Jones et al., 2010) can still be consumed by the consumers. In this proposed scenario, the value of MeJA application is significant without sacrificing the taste.

The feasibility of exogenous MeJA on broccoli and other Brassica vegetables might also change in different geological areas. As mentioned earlier, the bitter perception, or in general sensory perception, is a complex process. Diet habit, family food choice, and cultural difference will all affect taste and the food choices that people made (Costell, Tárrega, \& Bayarri, 2010). The tolerance toward certain sensorial quality can also be altered by these factors. Therefore, the acceptance of bitterness might change depending on the population, and MeJA-treated broccoli may be acceptable in different regions.

To have a better taste broccoli does not necessary mean to scarify the nutrition benefit of broccoli; however, this process usually takes a long time. For example, Beneforté $e^{\circledR}$ broccoli was bred to have significant higher amounts of glucoraphanin, which is the precursor of bioactive sulforaphane (Bell \& Wagstaff, 2017).The quinone reductase-inducing activity of sulforaphane was higher than other hydrolysis products (Ku et al., 2013b), and glucoraphanin and 
sulforaphane were not as bitter as other GSLs (sinigrin or progoitrin). The breeding process of Beneforté broccoli takes over 10 years. By contrast, MeJA application has a relatively low cost and is quick and easy since the application can be done toward the end of production. This application can be adapted in greenhouse or field conditions. With MeJA application, we can provide broccoli or other Brassica vegetables with greater health benefits, while Brassica breeders work on the long breeding process.

To further evaluate the effect of MeJA on nutritional quality of Brassica vegetables, other nutrients in plant, such as vitamins, minerals, and fiber, should be quantified to provide a more comprehensive aspect. For instance, it was reported that MeJA triggers the de novo biosynthesis of L-ascorbic acid (vitamin C) (Wolucka, Goossens, \& Inzé, 2005). Exogenous MeJA application affects sulfur assimilation or sulfur-related pathway (GS and sulfur volatiles in Chapter 4) as JA signaling involves in sulfur-assimilation. This relationship may change the cross-talk between sulfur and other minerals. For example, sulfur assimilation and glucosinolate biosynthesis was affected by selenium in broccoli (Kim \& Juvik, 2011; Tian, Hui, Thannhauser, Pan, \& Li, 2017). MeJA was reported to affect copper stress in development stage- and dosedependent manner in runner bean (Hanaka, Maksymiec, \& Bednarek, 2015), and to potential mediate free calcium concentration in cytosol (Szczegielniak et al., 2012). When the relationship between MeJA application and other nutrients in plants is investigated in detail, the feasibility of MeJA application may be re-evaluated with a more inclusive perspective.

In the future, neoglucobrassicin or neoglucobrassicin-derived hydrolysis products can be isolated from MeJA-treated Brasssica, and conducting descriptive tests, which provides a quantitative measure of bitterness that allows for comparison of intensity, should be performed. Molecular tool may be implemented to develop Brassica lines that contains only 
neoglucobrassicin or any GS of interest to facilitate GS extraction and bitterness intensity determination. In this way, bitterness level of neoglucobrassicin or neoglucobrassicin-derived hydrolysis products at different concentration can be estimated, which means bitterness level of broccoli and other Brassica vegetables can be estimated as well. Furthermore, this approach will useful to compare the bitterness between different GS, and help us understand the tolerant level of bitterness in Brassica vegetables of different populations as sensitivity to bitterness changes with age.

Meanwhile, the nutritional value of cooked MeJA-treated broccoli after the freezing procedure should be investigated, because precooked meals are usually frozen before the sale. Additional tests can be done on the mouthful texture of cooked MeJA-treated broccoli to determine a better MeJA-treated broccoli sensory profile. Research on the mechanism of the MeJA-mediated increase in the retention rate of GS should be extended by studying cellulose components or related gene expression as one potential way to investigate the higher retention of GS in MeJA-treated broccoli. MeJA application would not only be beneficial to the fresh vegetable industry, but could also benefit the processed food industry as well. For example, a focus group to discuss how MeJA application can be useful in ready-to-eat (RTE) food or processed food may be recruited and interests or concerns can be discussed from different professional groups.

Exogenous MeJA application has been shown to be an efficient plant growth regulator for inducing GS content in Brassica vegetables, and this application can also be used in a wide variety of crops for increasing different health-promoting compounds. Although pre-harvest MeJA application was shown to increase ethylene production and potentially decrease shelf-life, the application regime may be able to change depending on the crops or the storage condition. 
As 1-methylcyclopropene (1-MCP) application was reported to be more effective on a floral Brassica (Able, Wong, Prssad, \& O’Hare, 2002), the application of MeJA and 1-MCP together may enable the similar shelf-life and enhanced nutritional quality at the same time.

On the other hand, post-harvest MeJA application was shown to combat the unfavorable impacts of storage, such as pathogen attacks and chilling injuries, and to increase flavonoids in various fruits. In medicinal plants, MeJA is also shown to increase the bioactive compounds. For example, exogenous MeJA application was shown to elicit ginseng saponins (Kochan, Balcerczak, Lipert, Szymańska, \& Szymczyk, 2018) and artemisinin in Artemisia annua (Wu et al., 2011). The possibility of using MeJA as an effective elicitor to produce crops with high nutrition or pharmaceutical ingredients should not be underestimated, and future research may focus on the mechanism of MeJA-mediated pathways in different plants to better discover the potential of MeJA. 


\section{Supplementary Material}

Supplementary Table S1. Glucosinolate profile of different maturity (different leaf location) of 'Red Russian' kale. Stages were separated by the different position on the plant from apical to basal leaf.

\begin{tabular}{|c|c|c|c|c|c|c|c|c|c|c|c|}
\hline Stage & Glucoiberin & Glucoerucin & GR & Gluconapin & Progoitrin & Sinigrin & GBS & NeoGBS & 4MGBS & 4OHGBS & GNS \\
\hline 1 & $\begin{array}{c}0.42 \pm 0.25 \\
\mathrm{ab}\end{array}$ & $1.94 \pm 0.44 \mathrm{a}$ & $\begin{array}{c}7.59 \pm 1.38 \\
\mathrm{a}\end{array}$ & $1.40 \pm 0.52 \mathrm{a}$ & $\begin{array}{c}47.09 \pm 9.94 \\
\mathrm{a}\end{array}$ & $\begin{array}{c}0.37 \pm 0.14 \\
\mathrm{a}\end{array}$ & $\begin{array}{c}8.39 \pm 3.89 \\
\mathrm{a}\end{array}$ & $\begin{array}{c}6.79 \pm 2.33 \\
\mathrm{a}\end{array}$ & $\begin{array}{c}0.19 \pm 0.08 \\
\mathrm{a}\end{array}$ & $\begin{array}{c}1.52 \pm 0.46 \\
\mathrm{a}\end{array}$ & $\begin{array}{c}1.15 \pm 0.27 \\
\mathrm{a}\end{array}$ \\
\hline 2 & $0.42 \pm 0.05 \mathrm{a}$ & $0.67 \pm 0.19 \mathrm{~b}$ & $\begin{array}{c}5.61 \pm 0.96 \\
b\end{array}$ & $1.51 \pm 0.76 \mathrm{a}$ & $\begin{array}{c}24.04 \pm 8.15 \\
b\end{array}$ & $\begin{array}{c}0.16 \pm 0.05 \\
\mathrm{~b}\end{array}$ & $\begin{array}{c}3.37 \pm 1.16 \\
\mathrm{~b}\end{array}$ & $\begin{array}{c}1.74 \pm 0.61 \\
\mathrm{~b}\end{array}$ & $\begin{array}{c}0.06 \pm 0.02 \\
b\end{array}$ & $\begin{array}{c}0.46 \pm 0.08 \\
\mathrm{~b}\end{array}$ & $\begin{array}{c}0.86 \pm 0.10 \\
\mathrm{a}\end{array}$ \\
\hline 3 & $\begin{array}{c}0.28 \pm 0.17 \\
\mathrm{ab}\end{array}$ & $0.44 \pm 0.25 \mathrm{c}$ & $\begin{array}{c}1.84 \pm 1.59 \\
\mathrm{c}\end{array}$ & $1.42 \pm 0.05 \mathrm{a}$ & $\begin{array}{c}6.19 \pm 2.18 \\
c\end{array}$ & $\begin{array}{c}0.00 \pm 0.00 \\
\mathrm{c}\end{array}$ & $\begin{array}{c}0.73 \pm 0.44 \\
\mathrm{c}\end{array}$ & $\begin{array}{c}0.70 \pm 0.20 \\
\mathrm{~b}\end{array}$ & $\begin{array}{c}0.03 \pm 0.01 \\
\mathrm{~b}\end{array}$ & $\begin{array}{c}0.39 \pm 0.23 \\
\mathrm{~b}\end{array}$ & $\begin{array}{c}0.64 \pm 0.40 \\
b\end{array}$ \\
\hline 4 & $0.11 \pm 0.05 \mathrm{~b}$ & $0.37 \pm 0.14 \mathrm{bc}$ & $\begin{array}{c}0.25 \pm 0.15 \\
\mathrm{c}\end{array}$ & $0.51 \pm 0.13 \mathrm{~b}$ & $\begin{array}{c}1.91 \pm 0.43 \\
\mathrm{c}\end{array}$ & $\begin{array}{c}0.00 \pm 0.00 \\
\mathrm{c}\end{array}$ & $\begin{array}{c}0.17 \pm 0.13 \\
\mathrm{c}\end{array}$ & $\begin{array}{c}0.25 \pm 0.09 \\
\mathrm{~b}\end{array}$ & $\begin{array}{c}0.00 \pm 0.00 \\
\mathrm{~b}\end{array}$ & $\begin{array}{c}0.34 \pm 0.12 \\
\mathrm{~b}\end{array}$ & $\begin{array}{c}0.38 \pm 0.20 \\
\mathrm{~b}\end{array}$ \\
\hline 5 & $\begin{array}{c}0.35 \pm 0.21 \\
\mathrm{ab}\end{array}$ & $0.17 \pm 0.09 \mathrm{c}$ & $\begin{array}{c}0.13 \pm 0.07 \\
\mathrm{c}\end{array}$ & $0.22 \pm 0.11 \mathrm{~b}$ & $\begin{array}{c}1.01 \pm 0.70 \\
\mathrm{c}\end{array}$ & $\begin{array}{c}0.00 \pm 0.00 \\
\mathrm{c}\end{array}$ & $\begin{array}{c}0.42 \pm 0.23 \\
\mathrm{c}\end{array}$ & $\begin{array}{c}0.62 \pm 0.22 \\
\mathrm{~b}\end{array}$ & $\begin{array}{c}0.01 \pm 0.01 \\
\mathrm{~b}\end{array}$ & $\begin{array}{c}0.15 \pm 0.08 \\
\mathrm{~b}\end{array}$ & $\begin{array}{c}0.19 \pm 0.13 \\
\mathrm{~b}\end{array}$ \\
\hline
\end{tabular}

${ }^{\mathrm{z}}$ Values are means of four replications. Kale leaves were harvested at five different positions (stage 1: youngest, stage 2, stage 3, stage 4, and stage 5: oldest) from the same plant to different five maturation stages ${ }^{\mathrm{z}}$. Abbreviation: $\mathrm{GR}=$ glucoraphanin, GBS $=$ glucobrassicin, NeoGBS $=$ neoglubrassicin, 4MGRN = 4-methoxyglucobrassicin, 4OHBRN = 4-OH-glucobrassicin, GNS = gluconasturtiin. 
Supplementary Table S2. Tentative identification of desulfo-glucosinolate in 'Red Russian' kale by LC- high resolution MS in the full scan positive and negative ion mode.

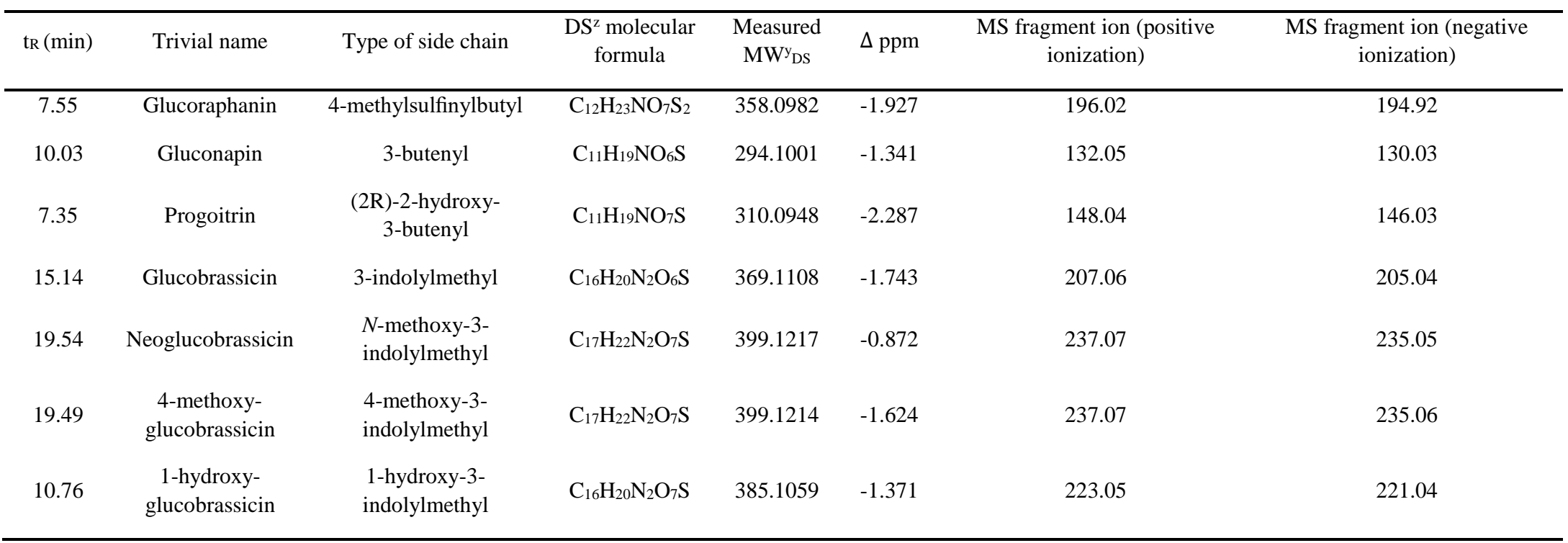

${ }^{\mathrm{z}} \mathrm{DS}$ indicates desulfo-glucosinolate. 
Supplementary Table S3. Primer set information for gene expression analysis

\begin{tabular}{|c|c|c|c|c|}
\hline Gene class & Gene name & Gene model & Type & Sequence \\
\hline \multirow[t]{4}{*}{ Chain elongation } & \multirow[t]{2}{*}{ MAM3 } & \multirow[t]{2}{*}{ Bol004799 } & Forward & GGTGGATGAAGAAACAGGTTACG \\
\hline & & & Reverse & CAAACCGCCTCGATGTCTCT \\
\hline & \multirow[t]{2}{*}{ SUR1 } & \multirow[t]{2}{*}{ Bol029775 } & Forward & GCTCCCACGTCCCGTTT \\
\hline & & & Reverse & GCGAACCTCGAGACCACTGT \\
\hline \multirow{8}{*}{$\begin{array}{l}\text { Core-structure } \\
\text { biosynthesis }\end{array}$} & \multirow[t]{2}{*}{ SOT17 } & \multirow[t]{2}{*}{ Bol030757 } & Forward & CCATCGCCACGCTTCCT \\
\hline & & & Reverse & CCGCCGTACTCGACGAAA \\
\hline & \multirow[t]{2}{*}{ SOT18 } & \multirow[t]{2}{*}{ Bol026202v2 } & Forward & CCCAAAGACAGGCACCACTT \\
\hline & & & Reverse & GGAATCGTCGAAGCGAGATC \\
\hline & \multirow[t]{2}{*}{ CYP79B2 } & \multirow[t]{2}{*}{ Bol032767 } & Forward & GATGAAATTAAACCCACCATTAAGGA \\
\hline & & & Reverse & GCCATGGCCCATTCGA \\
\hline & \multirow[t]{2}{*}{ SOT16 } & \multirow[t]{2}{*}{ Bol039395 } & Forward & TTCGACGACGCCACGAA \\
\hline & & & Reverse & CTCCACGTAAGGCACGAACTC \\
\hline \multirow{10}{*}{$\begin{array}{l}\text { Secondary } \\
\text { modification }\end{array}$} & \multirow[t]{2}{*}{$G S L-O H$} & \multirow[t]{2}{*}{ Bol033373 } & Forward & GCTTGTTGATGCTCTGTCATTGT \\
\hline & & & Reverse & TGGCGCCGAGCGTTAG \\
\hline & \multirow[t]{2}{*}{ CYP81F1 } & \multirow[t]{2}{*}{ Bol028913 } & Forward & CCGAGACATTCCGGCTATTC \\
\hline & & & Reverse & CATGTCCTCCGTCGGTCTTC \\
\hline & \multirow[t]{2}{*}{ CYP81F2 } & \multirow[t]{2}{*}{ Bol026044 } & Forward & TCTCCCACCAGGACCAACTC \\
\hline & & & Reverse & GGTGGACCGGCGGTTT \\
\hline & \multirow[t]{2}{*}{ CYP81F3 } & \multirow[t]{2}{*}{ Bol028919 } & Forward & CTCCTCACTCGCAACAGAATGT \\
\hline & & & Reverse & GGAAACAAGGGCGGTTTGAT \\
\hline & \multirow[t]{2}{*}{ CYP81F4 } & \multirow[t]{2}{*}{ Bol032712v2 } & Forward & TCCCTCTCCGCCTCACTCT \\
\hline & & & Reverse & GGTGGACGGGAGGTTTAATGA \\
\hline \multirow{8}{*}{$\begin{array}{l}\text { Transcription } \\
\text { factors }\end{array}$} & $M Y B 28$ & Bol036286 & Forward & TCTGAGCAGATTCTCAATGAAGATG \\
\hline & & & Reverse & TCAGGGTAAAACGTTGTTTGGA \\
\hline & MYB29 & Bol008849 & Forward & GCTTCCATGGGCAATATCATATC \\
\hline & & & Reverse & GACATGGAGGAGACAGTGTTGTAGA \\
\hline & MYB34 & Bol007760 & Forward & GCTCAAACCGGTGGCAAA \\
\hline & & & Reverse & CGTCAAGATCATCGGAGAAAGA \\
\hline & $M Y B 122$ & BoMYB122 & Forward & CTTCCCGACAAAGCTGGACT \\
\hline & & & Reverse & TTGGCTAAACTCACCACGCT \\
\hline Myrosinase & $T G G 1$ & Bol017328v2 & Forward & GTGCCTACGAGAGGCTATTCAAC \\
\hline & & & Reverse & GCCGTAACATCTTTCATCAACCT \\
\hline & $T G G 2$ & Bol028319v2 & Forward & CGAACTCAACGCTACTGGTTACA \\
\hline & & & Reverse & TACTCCCCTGCTCCTCTTTCC \\
\hline Specifier proteins & $E S P$ & Bol006378 & Forward & CTACACGACTGCTACCGTCTATGG \\
\hline & & & Reverse & GGTTGTTGGTGGGACGTTTT \\
\hline & ESM1 & Bol005067v2 & Forward & TCCGATGTTGAACCAGTTTGC \\
\hline & & & Reverse & CGAAGGATGGCGTTGTAGAAA \\
\hline Endogenous & Actin & Bol030974 & Forward & TCCCGAGAGGAAGTACAGTGTCT \\
\hline control gene & & & Reverse & GAGATCCACATCTGCTGGAATG \\
\hline
\end{tabular}


Supplementary Table S5. Tentative identification of volatiles in 'Green Magic' broccoli with or without $250 \mu \mathrm{M}$ methyl jasmonate treatment.

\begin{tabular}{|c|c|c|c|c|}
\hline Volatile compounds & $\begin{array}{l}\text { Retention } \\
\text { time (min) }\end{array}$ & $\begin{array}{c}\text { Identification } \\
\text { Method }\end{array}$ & $\begin{array}{c}\text { Odor } \\
\text { Descriptions }\end{array}$ & References $^{\mathrm{s}}$ \\
\hline Dimethyl sulfide & 2.85 & NIST $^{\mathrm{y}}$ & $\begin{array}{c}\text { cabbage, sulfur, } \\
\text { gasoline }\end{array}$ & Flavornet \\
\hline 2-Ethyl-furan & 4.16 & NIST, STD ${ }^{\mathrm{x}}$ & Sweet, earthy & $\begin{array}{c}\text { (Rajkumar et al., } \\
\text { 2017) }\end{array}$ \\
\hline Dimethyl disulfide & 5.05 & NIST & $\begin{array}{c}\text { onion, cabbage, } \\
\text { putrid }\end{array}$ & Flavornet \\
\hline Hexanal & 6.61 & NIST, STD & $\begin{array}{c}\text { grass, tallow, } \\
\text { fat }\end{array}$ & Flavornet \\
\hline (E)-2-hexenal & $8.13 \mathrm{f}$ & NIST, STD & green, leaf & Flavornet \\
\hline Dimethyl trisulfide & 10.88 & NIST, STD & $\begin{array}{l}\text { sulfur, fish, } \\
\text { cabbage }\end{array}$ & Flavornet \\
\hline Nonanal & 13.48 & NIST, STD & $\begin{array}{l}\text { fat, citrus, } \\
\text { green }\end{array}$ & Flavornet \\
\hline Dimethyl Tetrasulfide & 15.38 & NIST & cabbage, sulfur & Flavornet \\
\hline
\end{tabular}

${ }^{\mathrm{z}}$ The reference of odor descriptions

${ }^{\mathrm{y}}$ Comparison with the NIST library.

${ }^{\mathrm{x}}$ Authentic standard for identification using retention time and fragment pattern. 
Supplementary Table S6. Primary metabolites that high in variable influence on projection (VIP) by partial least square - discrimination analysis (PLS-DA) from raw and steamed 'Green Magic' broccoli with or without $250 \mu \mathrm{M}$ MeJA treatment bv GC-MS analysis.

\begin{tabular}{|c|c|c|c|c|c|}
\hline $\begin{array}{l}\text { Tentative } \\
\text { metabolites }\end{array}$ & $\begin{array}{l}\text { Retention } \\
\text { time } \\
\text { (min) }\end{array}$ & Characteristic ion $(\mathrm{m} / \mathrm{z})$ & $\mathrm{TMS}^{\mathrm{z}}$ & VIP & Identification \\
\hline Glucose $^{\mathrm{y}}$ & 12.42 & $\mathbf{7 3}^{\mathrm{x}}, 147,160,205,319$ & $\begin{array}{l}\text { Meox, } \\
(\mathrm{TMS})_{5}\end{array}$ & 8.11 & $\begin{array}{c}\text { (Chiu, Juvik, \& Ku, } \\
\text { 2018) }\end{array}$ \\
\hline Alanine & 5.6 & 73, 116, 147 & $(\mathrm{TMS})_{2}$ & 3.74 & $\begin{array}{c}\text { (Chiu, Juvik, \& Ku, } \\
\text { 2018) }\end{array}$ \\
\hline Glutamine & 11.53 & $73, \mathbf{1 5 6}, 245$ & $(\mathrm{TMS})_{3}$ & 3.42 & $\mathrm{STD} \mathrm{NIST}^{\mathrm{w}}$ \\
\hline Myo-Inositol & 13.73 & 73, 147, 217, 305, 318 & $(\mathrm{TMS})_{6}$ & 1.98 & STD/NIST \\
\hline Fructose & 12.3 & $73, \mathbf{1 0 3}, 147,217,307$ & $\begin{array}{l}\text { Meox, } \\
(\mathrm{TMS})_{5}\end{array}$ & 1.93 & $\begin{array}{c}\text { (Chiu, Juvik, \& Ku, } \\
\text { 2018) }\end{array}$ \\
\hline $\begin{array}{l}\text { Glutamic } \\
\text { acid }\end{array}$ & 10.36 & $73,128,246$ & $(\mathrm{TMS})_{3}$ & 1.89 & STD/NIST \\
\hline Leucine & 7.28 & $147, \mathbf{1 5 8}, 299$ & $(\mathrm{TMS})_{2}$ & 1.78 & STD/NIST \\
\hline $\begin{array}{l}\text { Quininic } \\
\text { acid }\end{array}$ & 12.16 & $147,255, \mathbf{3 4 5}$ & $(\mathrm{TMS})_{5}$ & 1.66 & NIST \\
\hline Sucrose & 16.8 & $73,147,217,271, \mathbf{3 6 1}$ & $(\mathrm{TMS})_{8}$ & 1.63 & $\begin{array}{c}\text { (Chiu, Juvik, \& Ku, } \\
\text { 2018) }\end{array}$ \\
\hline
\end{tabular}

${ }^{\mathrm{z}}$ Meox, methyloxime; TMS, trimethylsilyl.

${ }^{\mathrm{y}}$ Significant metabolite detected by ANOVA is label as bold

${ }^{\mathrm{x}}$ Highest peak is label as bold

${ }^{\mathrm{w}}$ Metabolites were identified using commercial standard compounds (STD) in comparison with the mass spectra in The National Institute of Standards and Technology (NIST) and retention time. 
Supplementary Table S6. Glucosinolate hydrolysis profile of raw and steamed broccoli with or without $250 \mu \mathrm{M}$ methyl jasmonate application (average $\pm \mathrm{SD}$ ). Compound identification were based on a previous publication (M. J. Kim, Chiu, Kim, et al., 2017) or by comparison with data in the National Institute of Standards and Technology (NIST) library.

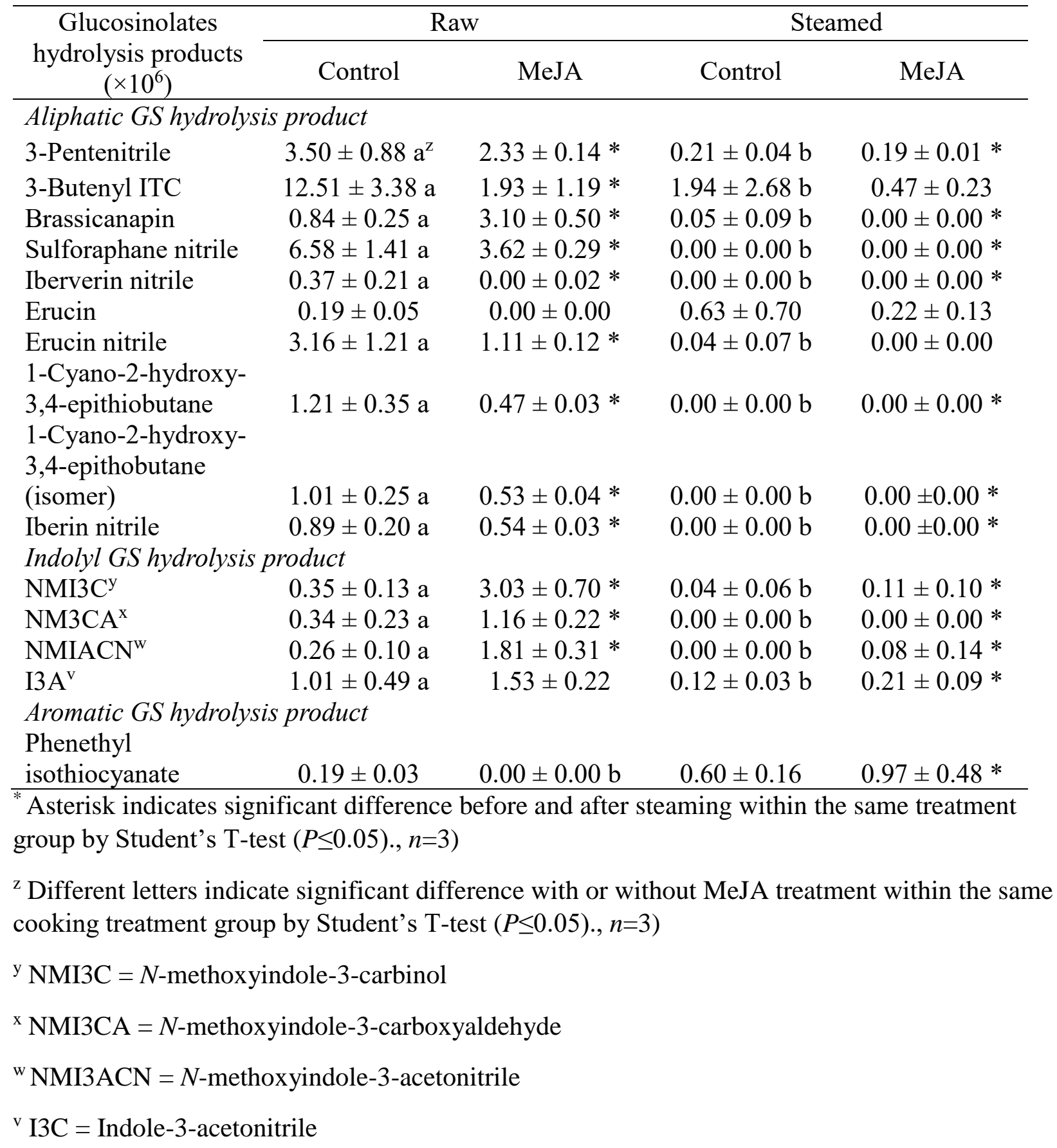


Supplementary Table S7. Results of two-wat ANOVA (MeJA treatment $\times$ cooking method) for (A) glucosinolate profiles of 2-minute cooking and (B) glucosinolate profiles of 5-minute cooking.

\begin{tabular}{|c|c|c|c|c|c|}
\hline \multicolumn{6}{|c|}{ Total Aliphatic GS } \\
\hline Source & $\begin{array}{l}\text { Number of } \\
\text { parameters }\end{array}$ & DF & $\begin{array}{l}\text { Sum of } \\
\text { Squares }\end{array}$ & F Ratio & Prob $>F$ \\
\hline MeJA treatment & 1 & 1 & 2.55258 & 2.0434 & 0.1721 \\
\hline Cooking & 3 & 3 & 79.07297 & 21.1 & $<.0001$ \\
\hline Cooking*MeJA & 3 & 3 & 9.954825 & 2.6564 & 0.0837 \\
\hline \multicolumn{6}{|l|}{ Total indolyl GS } \\
\hline Source & $\begin{array}{l}\text { Number of } \\
\text { parameters }\end{array}$ & $\mathrm{DF}$ & $\begin{array}{l}\text { Sum of } \\
\text { Squares }\end{array}$ & F Ratio & Prob $>F$ \\
\hline MeJA treatment & 1 & 1 & 3278.25 & 464.2703 & $<.0001$ \\
\hline Cooking & 3 & 3 & 639.8043 & 30.2033 & $<.0001$ \\
\hline Cooking*MeJA & 3 & 3 & 260.3897 & 12.2923 & 0.0002 \\
\hline \multicolumn{6}{|l|}{ Total GS } \\
\hline Source & $\begin{array}{l}\text { Number of } \\
\text { parameters }\end{array}$ & $\mathrm{DF}$ & $\begin{array}{l}\text { Sum of } \\
\text { Squares }\end{array}$ & F Ratio & Prob $>F$ \\
\hline MeJA treatment & 1 & 1 & 2790.209 & 319.1227 & $<.0001$ \\
\hline Cooking & 3 & 3 & 984.7455 & 37.5425 & $<.0001$ \\
\hline Cooking*MeJA & 3 & 3 & 353.2774 & 13.4684 & 0.0001 \\
\hline \multicolumn{6}{|c|}{ (B) } \\
\hline \multicolumn{6}{|c|}{ Total Aliphatic GS } \\
\hline Source & Nparm & DF & $\begin{array}{l}\text { Sum of } \\
\text { Squares }\end{array}$ & F Ratio & Prob $>F$ \\
\hline MeJA treatment & 1 & 1 & 2.552580 & 28.3724 & 0.1721 \\
\hline Cooking & 3 & 3 & 79.072965 & 19.1566 & $<.0001$ \\
\hline Cooking*MeJA & 3 & 3 & 9.954825 & 5.718 & 0.0834 \\
\hline \multicolumn{6}{|l|}{ Total indolyl GS } \\
\hline Source & Nparm & $\mathrm{DF}$ & $\begin{array}{l}\text { Sum of } \\
\text { Squares }\end{array}$ & F Ratio & Prob $>F$ \\
\hline MeJA treatment & 1 & 1 & 3278.25 & 464.2703 & $<.0001$ \\
\hline Cooking & 3 & 3 & 639.8043 & 30.2033 & $<.0001$ \\
\hline Cooking*MeJA & 3 & 3 & 260.3897 & 12.2923 & 0.0002 \\
\hline \multicolumn{6}{|l|}{ Total GS } \\
\hline Source & Nparm & DF & $\begin{array}{l}\text { Sum of } \\
\text { Squares }\end{array}$ & F Ratio & Prob $>F$ \\
\hline MeJA treatment & 1 & 1 & 3262.142 & 280.3611 & $<.0001$ \\
\hline Cooking & 3 & 3 & 1153.127 & 33.0347 & $<.0001$ \\
\hline Cooking*MeJA & 3 & 3 & 320.7688 & 9.1894 & 0.0009 \\
\hline
\end{tabular}


Supplementary Table S8. Glucosinolate profile ( $\mu$ mole $\cdot \mathrm{g}^{-1}$ dry weight) of (A) raw and 2-minute cooked broccoli and (B) raw and 5minute cooked broccoli with or without $250 \mu \mathrm{M}$ methyl jasmonate application (average $\pm \mathrm{SD}, \mathrm{n}=3$ ).

(A)

\begin{tabular}{|c|c|c|c|c|c|c|c|c|}
\hline \multirow{2}{*}{ Glucosinolate } & \multicolumn{2}{|c|}{ Raw } & \multicolumn{2}{|c|}{ 2-minute boiling } & \multicolumn{2}{|c|}{ 2-minute Steaming } & \multicolumn{2}{|c|}{ 2-minute Microwaving } \\
\hline & Control & MeJA & Control & MeJA & Control & MeJA & Control & MeJA \\
\hline Glucoraphanin & $6.60 \pm 0.58$ & $\begin{array}{l}4.66 \pm 0.50 \\
* * *\end{array}$ & $3.51 \pm 0.47$ & $\begin{array}{l}3.53 \pm 0.35 \\
\text { ns }\end{array}$ & $5.29 \pm 0.70$ & $\begin{array}{l}4.90 \pm 0.21 \\
\mathrm{~ns}\end{array}$ & $5.27 \pm 1.04$ & $\begin{array}{l}4.34 \pm 0.41 \\
\mathrm{~ns}\end{array}$ \\
\hline Progoitrin & $0.94 \pm 0.10$ & $1.11 \pm 0.11^{\mathrm{ns}}$ & $0.59 \pm 0.14$ & $\begin{array}{l}0.81 \pm 0.08 \\
\mathrm{~ns}\end{array}$ & $0.88 \pm 0.07$ & $\begin{array}{l}1.22 \pm 0.05 \\
* *\end{array}$ & $0.73 \pm 0.10$ & $\begin{array}{l}1.07 \pm 0.12 \\
*\end{array}$ \\
\hline Glucoerucin & $0.11 \pm 0.02$ & $\begin{array}{l}0.16 \pm 0.02 \\
*\end{array}$ & $0.06 \pm 0.01$ & $\begin{array}{l}0.07 \pm 0.01 \\
\mathrm{~ns}\end{array}$ & $0.10 \pm 0.01$ & $\begin{array}{l}0.16 \pm 0.01 \\
* * *\end{array}$ & $0.10 \pm 0.02$ & $\underset{\mathrm{ns}}{0.11 \pm 0.02}$ \\
\hline Glucoiberin & $1.13 \pm 0.10$ & $0.86 \pm 0.11^{\mathrm{ns}}$ & $0.89 \pm 0.20$ & $0.96 \pm 0.15^{\mathrm{ns}}$ & $0.92 \pm 0.15$ & $0.91 \pm 0.04^{\mathrm{ns}}$ & $0.91 \pm 0.20$ & $0.79 \pm 0.08^{\mathrm{ns}}$ \\
\hline Sinigrin & $0.30 \pm 0.02$ & $0.28 \pm 0.03^{\mathrm{ns}}$ & $0.17 \pm 0.03$ & $0.22 \pm 0.01^{\mathrm{ns}}$ & $0.29 \pm 0.03$ & $0.30 \pm 0.01^{\mathrm{ns}}$ & $0.26 \pm 0.05$ & $0.25 \pm 0.03^{\mathrm{ns}}$ \\
\hline $\begin{array}{l}\text { Total Aliphatic } \\
\text { GS }\end{array}$ & $9.07 \pm 0.76$ & $\begin{array}{l}7.06 \pm 0.75 \\
* * *\end{array}$ & $5.21 \pm 0.84$ & $\begin{array}{l}5.58 \pm 0.18 \\
n s\end{array}$ & $7.48 \pm 0.89$ & $\begin{array}{l}7.48 \pm 0.27 \\
n s\end{array}$ & $7.26 \pm 1.39$ & $\begin{array}{l}6.56 \pm 0.6 \\
6^{n s}\end{array}$ \\
\hline Glucobrassicin & $3.04 \pm 0.18$ & $\begin{array}{l}4.61 \pm 0.61 \\
* * *\end{array}$ & $1.80 \pm 0.03$ & $\begin{array}{l}3.57 \pm 0.12 \\
* * *\end{array}$ & $3.18 \pm 0.14$ & $\begin{array}{l}4.69 \pm 0.15 \\
* * *\end{array}$ & $2.74 \pm 0.49$ & $\begin{array}{l}4.65 \pm 0.33 \\
* * *\end{array}$ \\
\hline Neoglucobrassicin & $4.99 \pm 0.47$ & $\begin{array}{l}29.36 \pm 3.94 \\
* * *\end{array}$ & $2.20 \pm 0.08$ & $\begin{array}{l}14.14 \pm 0.32 \\
* * *\end{array}$ & $4.67 \pm 0.45$ & $\begin{array}{l}29.67 \pm 2.71 \\
* * *\end{array}$ & $4.04 \pm 0.69$ & $\begin{array}{l}28.21 \pm 3.46 \\
* * *\end{array}$ \\
\hline $\begin{array}{l}\text { 4-hydroxy- } \\
\text { glucobrassicin }\end{array}$ & $0.00 \pm 0.01$ & $\begin{array}{l}0.00 \pm 0.00 \\
\text { ns }\end{array}$ & $0.10 \pm 0.09$ & $\begin{array}{l}0.10 \pm 0.01 \\
\text { ns }\end{array}$ & $0.02 \pm 0.00$ & $\begin{array}{l}0.02 \pm 0.00 \\
\mathrm{~ns}\end{array}$ & $0.00 \pm 0.00$ & $\begin{array}{l}0.01 \pm 0.00 \\
\mathrm{~ns}\end{array}$ \\
\hline $\begin{array}{l}\text { 4-methoxy- } \\
\text { glucobrassicin }\end{array}$ & $0.49 \pm 0.04$ & $\begin{array}{l}0.59 \pm 0.07 \\
* *\end{array}$ & $0.29 \pm 0.01$ & $\begin{array}{l}0.40 \pm 0.02 \\
* *\end{array}$ & $0.51 \pm 0.01$ & $\begin{array}{l}0.63 \pm 0.02 \\
* * *\end{array}$ & $0.47 \pm 0.09$ & $\begin{array}{l}0.59 \pm 0.05 \\
\mathrm{~ns}\end{array}$ \\
\hline $\begin{array}{l}\text { Total indolyl } \\
\text { GS }\end{array}$ & $8.52 \pm 0.65$ & $\begin{array}{l}34.57 \pm 4.60 \\
* * *\end{array}$ & $4.39 \pm 0.15$ & $\begin{array}{l}18.21 \pm 0.45 \\
* * *\end{array}$ & $8.37 \pm 0.59$ & $\begin{array}{l}35.00 \pm 2.88 \\
* * *\end{array}$ & $7.25 \pm 1.22$ & $\begin{array}{l}33.46 \pm 3.66 \\
* * *\end{array}$ \\
\hline Gluconasturtiin & $0.09 \pm 0.01$ & $0.09 \pm 0.01$ & $0.09 \pm 0.02$ & $0.14 \pm 0.08$ & $0.10 \pm 0.01$ & $0.31 \pm 0.10$ & $0.07 \pm 0.01$ & $\begin{array}{l}0.09 \pm 0.01 \\
* * *\end{array}$ \\
\hline Total GS & $17.69 \pm 1.23$ & $\begin{array}{l}41.72 \pm 5.23 \\
* * *\end{array}$ & $9.69 \pm 0.73$ & $\begin{array}{l}23.93 \pm 0.32 \\
* * * *\end{array}$ & $15.95 \pm 0.41$ & $\begin{array}{l}42.80 \pm 3.00 \\
* * *\end{array}$ & $14.57 \pm 2.54$ & $\begin{array}{l}40.11 \pm 4.28 \\
* * *\end{array}$ \\
\hline
\end{tabular}


(B)

\begin{tabular}{|c|c|c|c|c|c|c|c|c|}
\hline \multirow{2}{*}{ Glucosinolate } & \multicolumn{2}{|c|}{ Raw } & \multicolumn{2}{|c|}{ 5-minute Boiling } & \multicolumn{2}{|c|}{ 5-minute Steaming } & \multicolumn{2}{|c|}{ 5-minute Microwaving } \\
\hline & Control & MeJA & Control & MeJA & Control & MeJA & Control & MeJA \\
\hline Glucoraphanin & $6.60 \pm 0.58$ & $\begin{array}{l}4.66 \pm 0.50 \\
* * *\end{array}$ & $2.82 \pm 0.18$ & $\begin{array}{l}2.28 \pm 0.38 \\
\mathrm{~ns}\end{array}$ & $6.01 \pm 0.05$ & $\begin{array}{l}4.93 \pm 0.60 \\
*\end{array}$ & $5.49 \pm 0.31$ & $\begin{array}{l}5.08 \pm 0.25 \\
\mathrm{~ns}\end{array}$ \\
\hline Progoitrin & $0.94 \pm 0.10$ & $\underset{\mathrm{ns}}{1.11 \pm 0.11}$ & $0.41 \pm 0.02$ & $\begin{array}{l}0.56 \pm 0.11 \\
\text { ns }\end{array}$ & $0.94 \pm 0.08$ & $\begin{array}{l}1.22 \pm 0.12 \\
* *\end{array}$ & $0.80 \pm 0.09$ & $\begin{array}{l}1.36 \pm 0.15 \\
* * *\end{array}$ \\
\hline Glucoerucin & $0.11 \pm 0.02$ & $0.16 \pm 0.02 *$ & $0.06 \pm 0.01$ & $\begin{array}{l}0.01 \pm 0.02 \\
* *\end{array}$ & $0.13 \pm 0.01$ & $\begin{array}{l}0.16 \pm 0.00 \\
* *\end{array}$ & $0.12 \pm 0.01$ & $\begin{array}{l}0.16 \pm 0.01 \\
* *\end{array}$ \\
\hline Glucoiberin & $1.13 \pm 0.10$ & $0.86 \pm 0.11^{\mathrm{ns}}$ & $0.65 \pm 0.06$ & $0.47 \pm 0.11^{\mathrm{ns}}$ & $1.07 \pm 0.01$ & $0.96 \pm 0.15^{\mathrm{ns}}$ & $1.02 \pm 0.08$ & $0.97 \pm 0.10^{\mathrm{ns}}$ \\
\hline Sinigrin & $0.30 \pm 0.02$ & $0.28 \pm 0.03^{\mathrm{ns}}$ & $0.13 \pm 0.01$ & $0.14 \pm 0.02^{\mathrm{ns}}$ & $0.32 \pm 0.04$ & $0.31 \pm 0.05^{\mathrm{ns}}$ & $0.29 \pm 0.02$ & $0.34 \pm 0.05^{\mathrm{ns}}$ \\
\hline $\begin{array}{l}\text { Total Aliphatic } \\
\text { GS }\end{array}$ & $9.07 \pm 0.76$ & $\begin{array}{l}7.06 \pm 0.75 \\
* *\end{array}$ & $4.06 \pm 0.22$ & $\begin{array}{l}3.46 \pm 0.63 \\
n s\end{array}$ & $7.02 \pm 2.52$ & $\begin{array}{l}7.58 \pm 1.11 \\
n s\end{array}$ & $7.72 \pm 0.47$ & $\begin{array}{l}7.91 \pm 0.55 \\
n s\end{array}$ \\
\hline Glucobrassicin & $3.04 \pm 0.18$ & $\begin{array}{l}4.61 \pm 0.61 \\
* * *\end{array}$ & $1.33 \pm 0.08$ & $\begin{array}{l}2.96 \pm 0.11 \\
* * *\end{array}$ & $2.89 \pm 0.11$ & $\begin{array}{l}4.98 \pm 0.22 \\
* * *\end{array}$ & $2.87 \pm 0.15$ & $\begin{array}{l}4.67 \pm 0.16 \\
* * *\end{array}$ \\
\hline Neoglucobrassicin & $4.99 \pm 0.47$ & $\begin{array}{l}29.36 \pm 3.94 \\
* * *\end{array}$ & $1.63 \pm 0.22$ & $\begin{array}{l}12.54 \pm 2.90 \\
* * *\end{array}$ & $4.05 \pm 0.42$ & $\begin{array}{l}31.07 \pm 2.28 \\
* * *\end{array}$ & $4.31 \pm 0.58$ & $\begin{array}{l}30.17 \pm 3.34 \\
* * *\end{array}$ \\
\hline $\begin{array}{l}\text { 4-hydroxy- } \\
\text { glucobrassicin }\end{array}$ & $0.00 \pm 0.01$ & $\begin{array}{l}0.00 \pm 0.00 \\
\text { ns }\end{array}$ & $0.04 \pm 0.01$ & $\begin{array}{l}0.06 \pm 0.05 \\
\text { ns }\end{array}$ & $0.02 \pm 0.01$ & $\begin{array}{l}0.02 \pm 0.00 \\
\text { ns }\end{array}$ & $0.00 \pm 0.00$ & $\begin{array}{l}0.01 \pm 0.00 \\
* *\end{array}$ \\
\hline $\begin{array}{l}\text { 4-methoxy- } \\
\text { glucobrassicin }\end{array}$ & $0.49 \pm 0.04$ & $\begin{array}{l}0.59 \pm 0.07 \\
* *\end{array}$ & $0.22 \pm 0.02$ & $\begin{array}{l}0.30 \pm 0.01 \\
* *\end{array}$ & $0.46 \pm 0.03$ & $\begin{array}{l}0.60 \pm 0.02 \\
* *\end{array}$ & $0.46 \pm 0.03$ & $\begin{array}{l}0.62 \pm 0.06 \\
* *\end{array}$ \\
\hline Total indole GS & $8.52 \pm 0.65$ & $\begin{array}{l}34.57 \pm 4.60 \\
* * *\end{array}$ & $3.22 \pm 0.31$ & $\begin{array}{l}15.86 \pm 2.90 \\
* * *\end{array}$ & $7.43 \pm 0.56$ & $\begin{array}{l}36.67 \pm 2.39 \\
* * *\end{array}$ & $7.24 \pm 0.79$ & $\begin{array}{l}35.46 \pm 3.47 \\
* * *\end{array}$ \\
\hline Gluconasturtiin & $0.09 \pm 0.01$ & $\begin{array}{l}0.09 \pm 0.01 \\
\text { ns }\end{array}$ & $0.09 \pm 0.01$ & $\begin{array}{l}0.06 \pm 0.02 \\
\text { ns }\end{array}$ & $0.11 \pm 0.01$ & $\begin{array}{l}0.19 \pm 0.01 \\
* * *\end{array}$ & $0.09 \pm 0.02$ & $\begin{array}{l}0.17 \pm 0.05 \\
* * *\end{array}$ \\
\hline Total GS & $17.69 \pm 1.23$ & $\begin{array}{l}41.72 \pm 5.23 \\
* * *\end{array}$ & $7.37 \pm 0.46$ & $\begin{array}{l}19.38 \pm 2.27 \\
* * *\end{array}$ & $13.29 \pm 4.75$ & $\begin{array}{l}44.43 \pm 3.45 \\
* * *\end{array}$ & $15.44 \pm 1.02$ & $\begin{array}{l}43.54 \pm 3.64 \\
* * * *\end{array}$ \\
\hline
\end{tabular}

Asterisk (*) indicates significant difference with or without MeJA within the same cooking treatment by Student's T-test $(P \leq 0.05$, $N=3$ ) with the significant interaction between MeJA treatment and cooking treatment detected (Supplementary Table S1).

ns, not significant; *, $P \leq 0.05 ;{ }^{* *}, P \leq 0.01$; ***, $P \leq 0.001$. 
Supplementary Table S9. Two-way analysis of variance (ANOVA) of the electrical conductivity in cooking water of broccoli with or without $250 \mu \mathrm{M}$ MeJA treatment.

2- min cooking

\begin{tabular}{lccccc}
\hline Source & Nparm & DF & $\begin{array}{c}\text { Sum of } \\
\text { Squares }\end{array}$ & F Ratio & Prob > F \\
MeJA treatment & 1 & 1 & 254.4 & 0.5095 & 0.489 \\
Cooking & 2 & 2 & 2709778.6 & 2713.816 & $<.0001$ \\
Cooking*MeJA & 2 & 2 & 768.7 & 0.7698 & 0.4847 \\
\hline 5-min cooking & Nparm & DF & Sum of & F Ratio & Prob > F \\
Source & 1 & & Squares & & \\
MeJA treatment & 2 & 1 & 12675.9 & 10.7719 & 0.0066 \\
Cooking & 2 & 2 & 4322252 & 1836.512 & $<.0001$ \\
Cooking*MeJA & 2 & 6115.6 & 2.5985 & 0.1154 \\
\hline
\end{tabular}


Supplementary Table S10. The peak intensity ( $\times 103)$ of glucosinolate hydrolysis products in (A) raw and 2-minute cooked and (B) raw and 5-minute cooked "Green Magic" broccoli with or without $250 \mu \mathrm{M}$ methyl jasmonate application (average $\pm \mathrm{SD}, \mathrm{n}=3$ ). Compound identification were based on a previous publication (Kim et al., 2017) or by comparison with data in the National Institute of Standards and Technology (NIST) library.

(A)

\begin{tabular}{|c|c|c|c|c|c|c|c|c|}
\hline & \multicolumn{2}{|c|}{ Raw } & \multicolumn{2}{|c|}{ 2-minute Boiling } & \multicolumn{2}{|c|}{ 2-minute Steaming } & \multicolumn{2}{|c|}{ 2-minute Microwaving } \\
\hline & Control & MeJA & Control & MeJA & Control & MeJA & Control & MeJA \\
\hline Sulforaphane & $0.10 \pm 0.02$ & $0.39 \pm 0.19^{\mathrm{ns}}$ & $0.08 \pm 0.07$ & $0.27 \pm 0.13^{\mathrm{ns}}$ & $0.12 \pm 0.12$ & $0.20 \pm 0.17^{\mathrm{ns}}$ & $0.10 \pm 0.09$ & $0.11 \pm 0.07^{\mathrm{ns}}$ \\
\hline $\begin{array}{l}\text { Sulforaphane } \\
\text { nitrile }\end{array}$ & $4.37 \pm 1.03$ & $4.58 \pm 1.48^{\mathrm{ns}}$ & $5.47 \pm 0.39$ & $3.51 \pm 0.95^{\mathrm{ns}}$ & $26.02 \pm 30.61$ & $3.84 \pm 0.73 *$ & $8.60 \pm 6.99$ & $2.36 \pm 1.78^{\mathrm{ns}}$ \\
\hline Erucin nitrile & $1.25 \pm 0.75$ & $0.67 \pm 0.81^{\mathrm{ns}}$ & $0.28 \pm 0.34$ & $1.83 \pm 2.66^{\mathrm{ns}}$ & $0.38 \pm 0.33$ & $0.31 \pm 0.19^{\mathrm{ns}}$ & $0.17 \pm 0.18$ & $3.13 \pm 1.17^{* *}$ \\
\hline $\begin{array}{l}\text { 1-cyano-3,4 } \\
\text { epithiobutene }\end{array}$ & $0.78 \pm 0.43$ & $0.37 \pm 0.09^{\mathrm{ns}}$ & $0.39 \pm 0.26$ & $0.60 \pm 0.20^{\mathrm{ns}}$ & $0.73 \pm 0.77$ & $0.29 \pm 0.05^{\mathrm{ns}}$ & $0.91 \pm 0.70$ & $0.33 \pm 0.14^{\mathrm{ns}}$ \\
\hline $\begin{array}{l}\text { 1-Cyano-2- } \\
\text { hydroxy-3,4- } \\
\text { epithiobutane }\end{array}$ & $0.82 \pm 0.42$ & $2.16 \pm 0.55^{* *}$ & $1.46 \pm 1.24$ & $0.67 \pm 0.25^{\mathrm{ns}}$ & $0.98 \pm 0.83$ & $0.72 \pm 0.12^{\mathrm{ns}}$ & $0.59 \pm 0.10$ & $0.55 \pm 0.31^{\mathrm{ns}}$ \\
\hline NMI3CA & $35.00 \pm 11.30$ & $\begin{array}{c}116.13 \pm 38.96 \\
* * *\end{array}$ & $5.89 \pm 0.76$ & $13.70 \pm 3.36^{\mathrm{ns}}$ & $22.75 \pm 5.79$ & $35.26 \pm 8.82^{\mathrm{ns}}$ & $2.86 \pm 0.83$ & $7.25 \pm 0.38^{\mathrm{ns}}$ \\
\hline NMI3CAN & $0.34 \pm 0.33$ & $0.65 \pm 0.17^{* * *}$ & $0.29 \pm 0.22$ & $0.69 \pm 0.30^{\mathrm{ns}}$ & $0.49 \pm 0.14$ & $0.44 \pm 0.12^{\mathrm{ns}}$ & $0.49 \pm 0.10$ & $0.15 \pm 0.18^{* *}$ \\
\hline NMI3C & $450.48 \pm 39.56$ & $\begin{array}{c}3991.30 \pm \\
539.36^{* * *}\end{array}$ & $65.76 \pm 8.96$ & $\begin{array}{c}53.09 \pm 47.95 \\
\text { ns }\end{array}$ & $106.48 \pm 95.03$ & $4.13 \pm 5.58^{\mathrm{ns}}$ & $523.48 \pm 67.09$ & $\begin{array}{c}33.30 \pm 31.54 \\
* *\end{array}$ \\
\hline $\begin{array}{l}\text { Indole-3- } \\
\text { acetonitrile }\end{array}$ & $0.21 \pm 0.14$ & $0.26 \pm 0.36^{\mathrm{ns}}$ & $0.25 \pm 0.24$ & $0.48 \pm 0.05^{\mathrm{ns}}$ & $0.78 \pm 0.35$ & $1.89 \pm 1.11^{* *}$ & $0.31 \pm 0.03$ & $0.44 \pm 0.16^{\mathrm{ns}}$ \\
\hline $\begin{array}{l}\text { Indole-3- } \\
\text { carbinol }\end{array}$ & $0.23 \pm 0.12$ & $0.93 \pm 0.13^{* * *}$ & $0.33 \pm 0.15$ & $0.67 \pm 0.20 *$ & $0.34 \pm 0.22$ & $0.45 \pm 0.19^{\mathrm{ns}}$ & $0.21 \pm 0.17$ & $0.22 \pm 0.11^{\mathrm{ns}}$ \\
\hline $\begin{array}{l}\text { Phenylethyl } \\
\text { ITC }\end{array}$ & $0.44 \pm 0.23$ & $1.23 \pm 0.26$ & $3.80 \pm 0.18$ & $1.80 \pm 0.40^{\mathrm{ns}}$ & $2.13 \pm 2.74$ & $3.43 \pm 1.31^{\mathrm{ns}}$ & $1.97 \pm 1.22$ & $1.83 \pm 1.51^{\mathrm{ns}}$ \\
\hline $\begin{array}{c}\text { Total Peak } \\
\text { Intensitv }\end{array}$ & $\begin{array}{c}494.85 \pm \\
5028\end{array}$ & $\begin{array}{l}4120.84 \pm \\
57974 * * *\end{array}$ & $\begin{array}{c}83.72 \pm \\
820\end{array}$ & $\begin{array}{l}77.72 \pm \\
4780^{\mathrm{ns}}\end{array}$ & $\begin{array}{c}163.12 \pm \\
83.80\end{array}$ & $\begin{array}{l}51.59 \pm \\
1222^{\mathrm{ns}}\end{array}$ & $\begin{array}{c}49.66 \pm \\
36.61\end{array}$ & $\begin{array}{c}540.69 \pm \\
7006 * * *\end{array}$ \\
\hline
\end{tabular}


(B)

\begin{tabular}{|c|c|c|c|c|c|c|c|c|}
\hline & \multicolumn{2}{|c|}{ Raw } & \multicolumn{2}{|c|}{ 5-minute Boiling } & \multicolumn{2}{|c|}{ 5-minute Steaming } & \multicolumn{2}{|c|}{ 5-minute Microwaving } \\
\hline & Control & MeJA & Control & MeJA & Control & MeJA & Control & MeJA \\
\hline Sulforaphane & $0.10 \pm 0.02$ & $0.39 \pm 0.19$ & $0.05 \pm 0.07$ & $0.12 \pm 0.10^{* *}$ & $0.14 \pm 0.08$ & $0.23 \pm 0.15$ & $0.17 \pm 0.06$ & $0.20 \pm 0.05$ \\
\hline $\begin{array}{c}\text { Sulforaphane } \\
\text { nitrile }\end{array}$ & $4.37 \pm 1.03$ & $4.58 \pm 1.48^{\mathrm{ns}}$ & $3.64 \pm 0.39$ & $9.31 \pm 3.78 * *$ & $4.40 \pm 2.10$ & $3.45 \pm 0.98^{\mathrm{ns}}$ & $8.00 \pm 6.96$ & $4.76 \pm 1.48^{\mathrm{ns}}$ \\
\hline Erucin nitrile & $1.25 \pm 0.75$ & $0.67 \pm 0.81^{\mathrm{ns}}$ & $0.83 \pm 0.34$ & $0.86 \pm 0.97^{\mathrm{ns}}$ & $0.58 \pm 0.25$ & $0.50 \pm 0.25^{\mathrm{ns}}$ & $0.45 \pm 0.27$ & $0.31 \pm 0.14^{\mathrm{ns}}$ \\
\hline $\begin{array}{c}\text { 1-cyano-3,4 } \\
\text { epithiobutene }\end{array}$ & $0.78 \pm 0.43$ & $0.37 \pm 0.09^{\mathrm{ns}}$ & $0.34 \pm 0.26$ & $0.63 \pm 0.20^{\mathrm{ns}}$ & $0.34 \pm 0.18$ & $0.08 \pm 0.06^{\mathrm{ns}}$ & $0.31 \pm 0.23$ & $0.23 \pm 0.24^{\mathrm{ns}}$ \\
\hline $\begin{array}{l}\text { 1-Cyano-2- } \\
\text { hydroxy-3,4- } \\
\text { epithiobutane }\end{array}$ & $0.82 \pm 0.42$ & $\begin{array}{l}2.16 \pm 0.55 \\
* * *\end{array}$ & $1.15 \pm 0.26$ & $0.65 \pm 0.20^{\mathrm{ns}}$ & $0.38 \pm 0.18$ & $0.70 \pm 0.06^{\mathrm{ns}}$ & $0.34 \pm 0.23$ & $0.75 \pm 0.24^{\mathrm{ns}}$ \\
\hline $\mathrm{NMI} \mathrm{CA}^{\dagger}$ & $\begin{array}{l}35.00 \pm \\
11.30\end{array}$ & $\begin{array}{l}116.13 \pm \\
38.96^{*}\end{array}$ & $3.82 \pm 0.76$ & $15.69 \pm 4.73^{\mathrm{ns}}$ & $56.13 \pm 25.34$ & $78.29 \pm 41.51 *$ & $28.35 \pm 10.68$ & $\begin{array}{l}101.45 \pm \\
46.64 * *\end{array}$ \\
\hline $\mathrm{NMI} 3 \mathrm{ACN} \ddagger$ & $0.34 \pm 0.33$ & $\begin{array}{l}0.65 \pm 0.17 \\
* * *\end{array}$ & $0.54 \pm 0.22$ & $0.25 \pm 0.42^{\mathrm{ns}}$ & $0.60 \pm 0.58$ & $0.77 \pm 1.23^{\mathrm{ns}}$ & $0.55 \pm 0.45$ & $0.46 \pm 0.42 * *$ \\
\hline NMI3C § & $\begin{array}{l}450.48 \pm \\
39.56\end{array}$ & $\begin{array}{l}3991.30 \pm \\
539.36^{* * *}\end{array}$ & $599.27 \pm 8.96$ & $\begin{array}{l}812.66 \pm \\
371.75^{\mathrm{ns}}\end{array}$ & $\begin{array}{l}135.23 \pm \\
231.83\end{array}$ & $\begin{array}{l}182.61 \pm \\
55.04 * *\end{array}$ & $\begin{array}{l}2361.27 \pm \\
1006.49\end{array}$ & $\begin{array}{l}180.02 \pm 114.76 \\
* *\end{array}$ \\
\hline $\begin{array}{l}\text { Indole-3- } \\
\text { acetonitrile }\end{array}$ & $0.21 \pm 0.14$ & $0.26 \pm 0.36^{\mathrm{ns}}$ & $0.37 \pm 0.24$ & $0.23 \pm 0.30^{\mathrm{ns}}$ & $0.61 \pm 0.45$ & $3.66 \pm 5.01^{\mathrm{ns}}$ & $0.23 \pm 0.23$ & $0.51 \pm 0.20^{\mathrm{ns}}$ \\
\hline $\begin{array}{c}\text { Indole-3- } \\
\text { carbinol }\end{array}$ & $0.23 \pm 0.12$ & $\begin{array}{l}0.93 \pm 0.13 \\
* * *\end{array}$ & $0.36 \pm 0.15$ & $0.33 \pm 0.09^{\mathrm{ns}}$ & $0.39 \pm 0.23$ & $0.25 \pm 0.11^{\mathrm{ns}}$ & $0.31 \pm 0.13$ & $0.72 \pm 0.41 *$ \\
\hline $\begin{array}{l}\text { Phenylethyl } \\
\text { ITC }\end{array}$ & $0.44 \pm 0.23$ & $1.23 \pm 0.26^{\mathrm{ns}}$ & $3.19 \pm 0.18$ & $1.24 \pm 0.48^{\mathrm{ns}}$ & $4.08 \pm 2.36$ & $3.37 \pm 1.75^{*}$ & $3.04 \pm 2.85$ & $2.06 \pm 0.92^{\mathrm{ns}}$ \\
\hline Total Peak & $494.43 \pm$ & $4119.09 \pm$ & $614.72 \pm$ & $842.64 \pm$ & $204.44 \pm$ & $274.67 \pm$ & $222.11 \pm$ & $2473.47 \pm$ \\
\hline Intensity & 50.06 & $579.83 * * *$ & 8.20 & $371.75^{\mathrm{ns}}$ & 234.45 & $78.81^{\mathrm{ns}}$ & 100.33 & $1054.43 *$ \\
\hline
\end{tabular}

${ }^{\dagger} \mathrm{NMI} 3 \mathrm{CA}==N$-methoxyindole-3- carboxyaldehyde; ${ }^{\dagger} \mathrm{NMI} 3 \mathrm{CN}=N$-methoxyindole-3-acetonitrile; ${ }^{\S} \mathrm{NMI} \mathrm{C}=N$-methoxyindole-3carbinol. Asterisk $\left(^{*}\right)$ indicates significant difference with or without MeJA within the same cooking treatment by Student's T-test $(P \leq 0.05, N=3)$.

ns , not significant; *, $P \leq 0.05 ; * *, P \leq 0.01 ; * * *, P \leq 0.001$. 
Supplementary Table S11. Primary metabolites that high in variable influence on projection (VIP) by partial least square - discrimination analysis (PLS-DA) from raw and cooked 'Green Magic' broccoli with or without $250 \mu \mathrm{M}$ MeJA treatment by GC-MS analysis. All listed metabolite were significant changed by ANOVA at $P \leq 0.05$.

\begin{tabular}{|c|c|c|c|c|c|}
\hline $\begin{array}{l}\text { Tentative } \\
\text { metabolites }\end{array}$ & $\begin{array}{l}\text { Retention } \\
\text { time } \\
\text { (min) }\end{array}$ & Characteristic ion $(\mathrm{m} / \mathrm{z})$ & TMS & VIP & Identification \\
\hline Oxoproline & 9.57 & $73,147, \mathbf{1 5 6}^{\dagger}$ & $(\mathrm{TMS})_{2}$ & 5.94 & NIST \\
\hline $\begin{array}{l}\text { Glutamic } \\
\text { acid }\end{array}$ & 10.36 & $73,128,246$ & $(\mathrm{TMS})_{3}$ & 1.89 & $\mathrm{STD} / \mathrm{NIST}^{\mathrm{w}}$ \\
\hline Valine & 6.75 & $73, \mathbf{1 4 4}, 218$ & $(\mathrm{TMS})_{2}$ & 1.69 & STD/NIST \\
\hline Proline & 7.58 & $73, \mathbf{1 4 2}, 216$ & $(\mathrm{TMS})_{2}$ & 2.85 & STD/NIST \\
\hline Serine & 7.17 & $73, \mathbf{1 1 6}, 132$ & $(\mathrm{TMS})_{2}$ & 1.97 & STD/NIST \\
\hline Isoleucine & 7.52 & $73, \mathbf{1 5 8}, 218$ & $(\mathrm{TMS})_{2}$ & 2.09 & STD/NIST \\
\hline Alanine & 5.60 & $73, \mathbf{1 1 6}, 147$ & $(\mathrm{TMS})_{2}$ & 2.70 & $\begin{array}{c}\text { (Chiu, Juvik, \& } \\
\text { Ku, 2018) }\end{array}$ \\
\hline Fructose & 12.3 & $73, \mathbf{1 0 3}, 147,217,307$ & Meox, $(\mathrm{TMS})_{5}$ & 1.93 & $\begin{array}{l}\text { (Chiu, Juvik, \& } \\
\text { Ku, 2018) }\end{array}$ \\
\hline Glucose $^{\mathrm{y}}$ & 12.42 & $\mathbf{7 3}, 147,160,205,319$ & Meox, $(\mathrm{TMS})_{5}$ & 8.11 & $\begin{array}{c}\text { (Chiu, Juvik, \& } \\
\text { Ku, 2018) }\end{array}$ \\
\hline Sucrose & 16.80 & $73,147,217,271, \mathbf{3 6 1}$ & $(\mathrm{TMS})_{8}$ & 1.63 & $\begin{array}{c}\text { (Chiu, Juvik, \& } \\
\text { Ku, 2018) }\end{array}$ \\
\hline Myo-Inositol & 13.73 & $\begin{array}{l}\text { 73, 147, 217, 305, } 318 \\
147255 \mathbf{3 4 5}\end{array}$ & $(\mathrm{TMS})_{6}$ & 1.98 & STD/NIST \\
\hline
\end{tabular}

${ }^{\dagger}$ Highest peak is label as bold

‡ Meox, methyloxime; TMS, trimethylsilyl.

$\S$ Metabolites were identified using commercial standard compounds (STD) in comparison with the mass spectra in The National Institute of Standards and Technology (NIST) and retention time. 

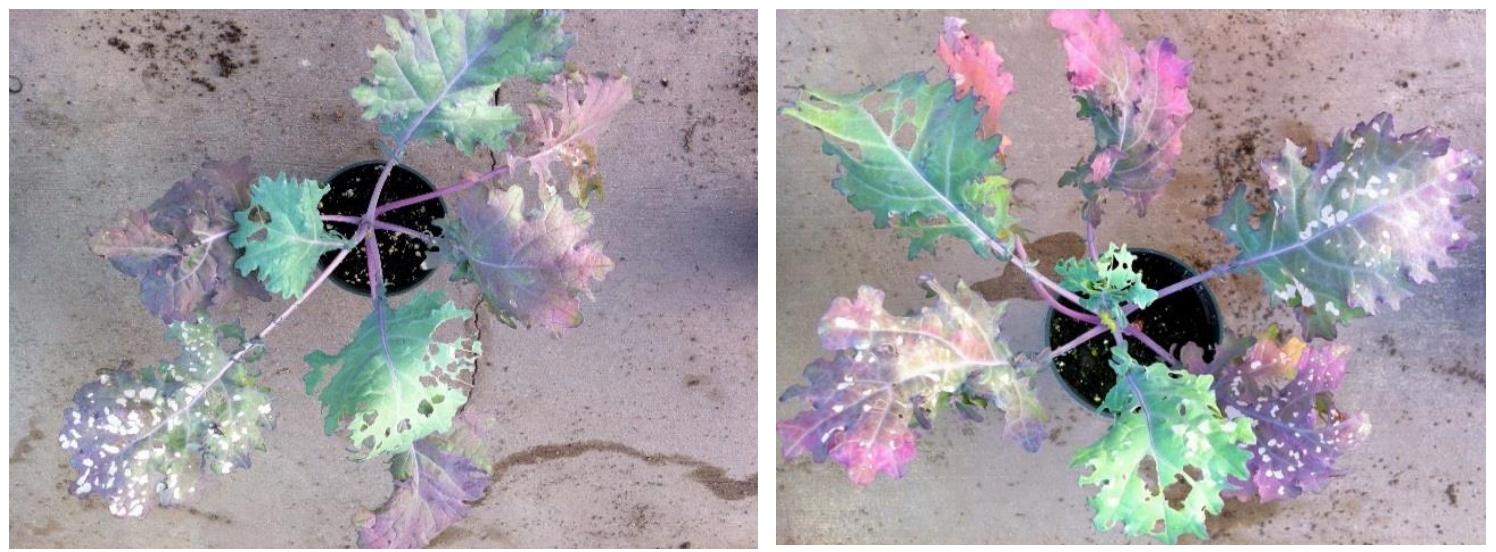

Supplementary Figure S1. Representative image of insect damaged kale seedlings. 


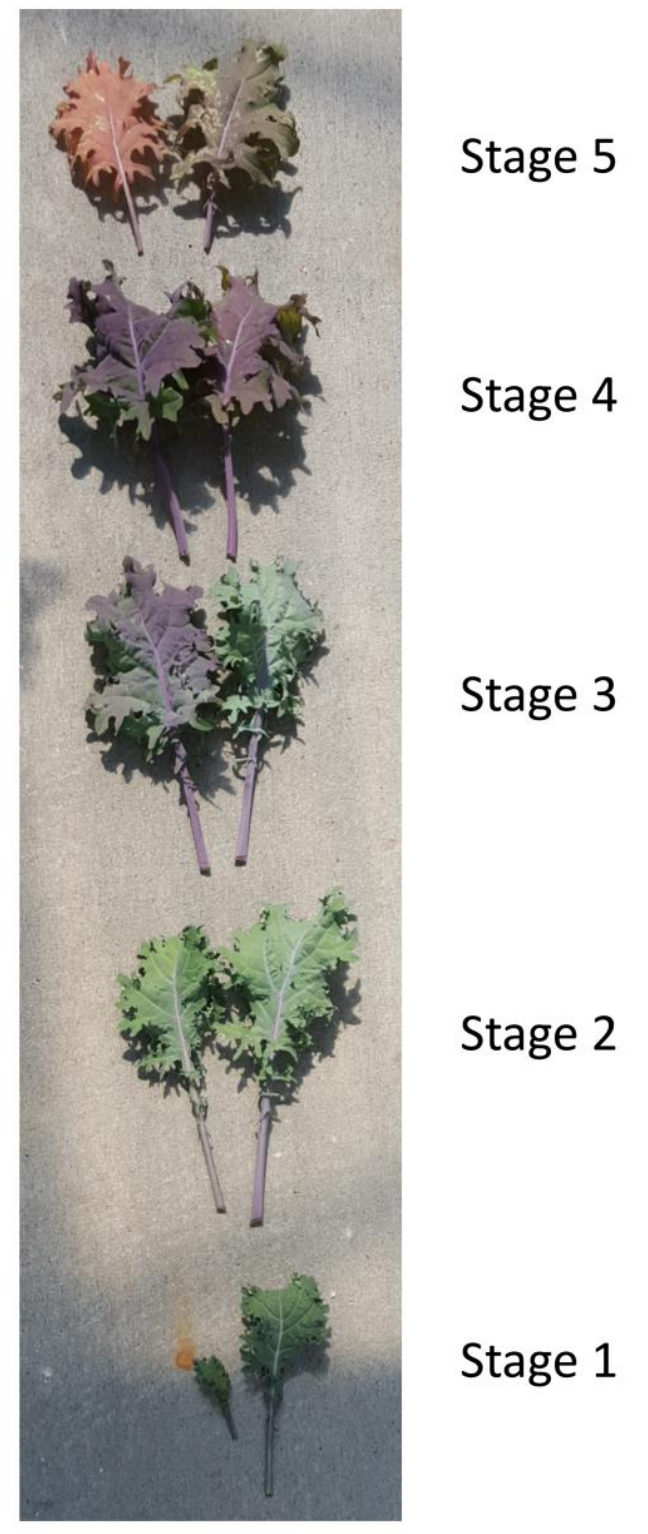

Supplementary Figure S2. Representative image of different leaf location (maturation stage) in kale seedling. (Stage 1: youngest - Stage 5: oldest). 
(A)

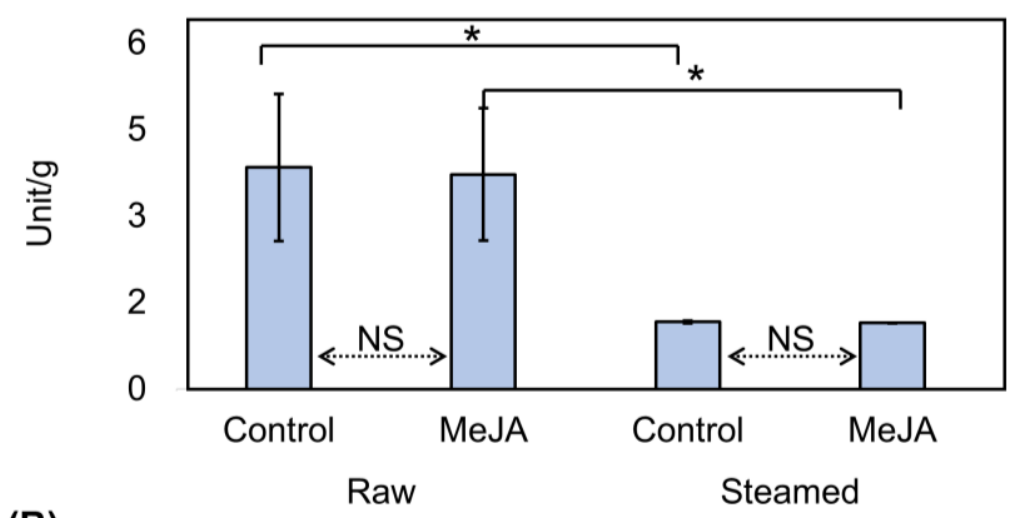

(B)

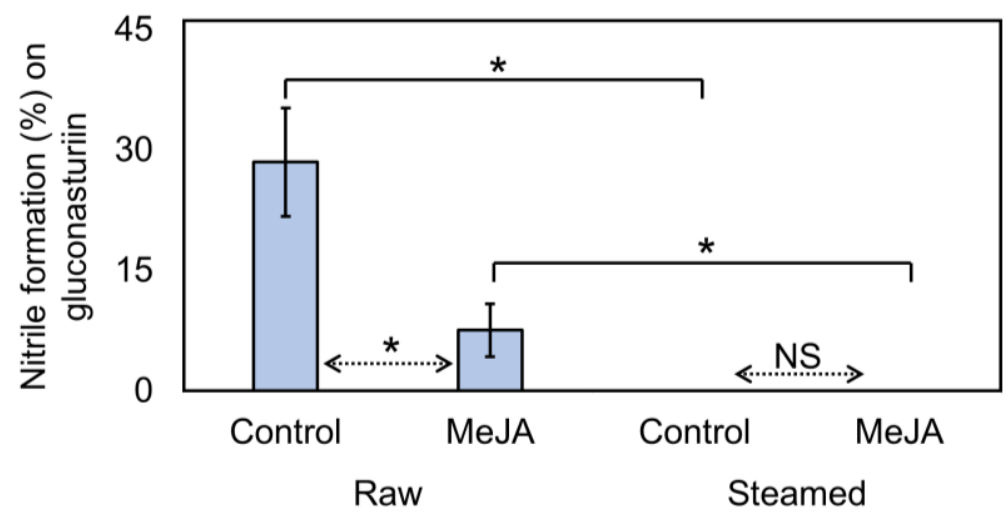

(C)

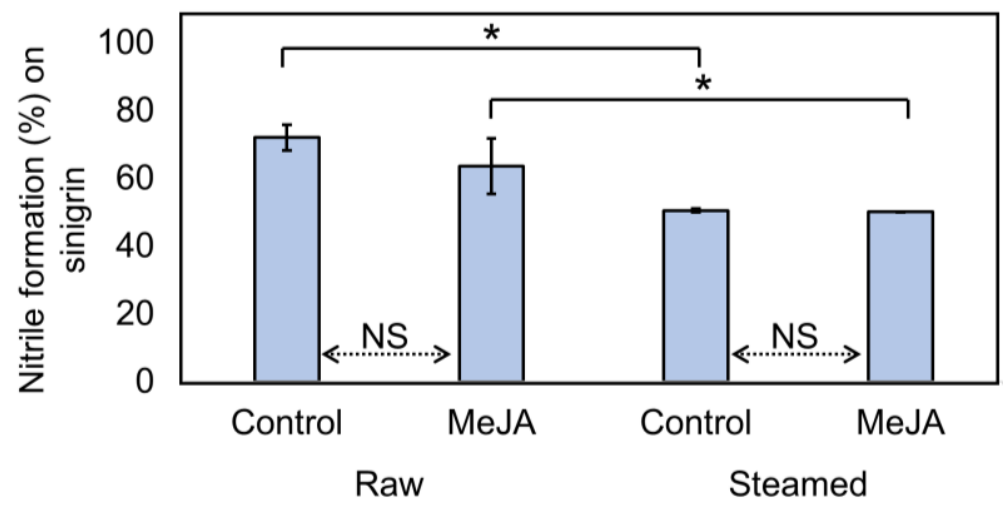

Supplementary Figure S3. Myrosinase activity (A), nitrile formation from sinigrin (B), and nitrile formation from gluconasturiin (C) of raw and steamed 'Green Magic' broccoli with or without $250 \mu \mathrm{M}$ methyl jasmonate application. Asterisk indicates the significant difference with or without $250 \mu \mathrm{M}$ MeJA treatment and before and after steaming by Student's T-test $(P \leq 0.05$, $n=3$ ). NS indicated not significant difference with or without $250 \mu \mathrm{M}$ methyl jasmonate application. 
(A)

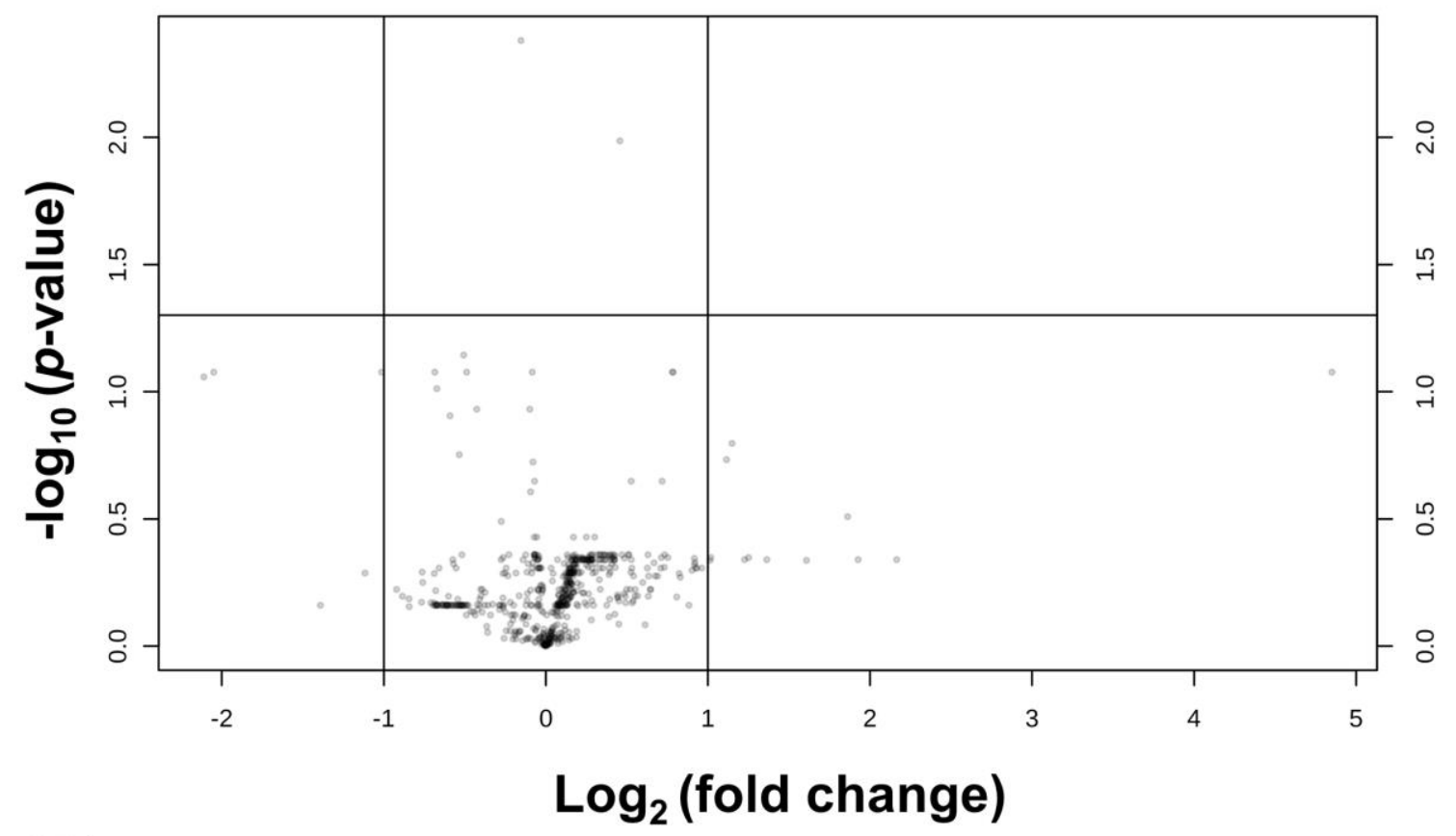

(B)

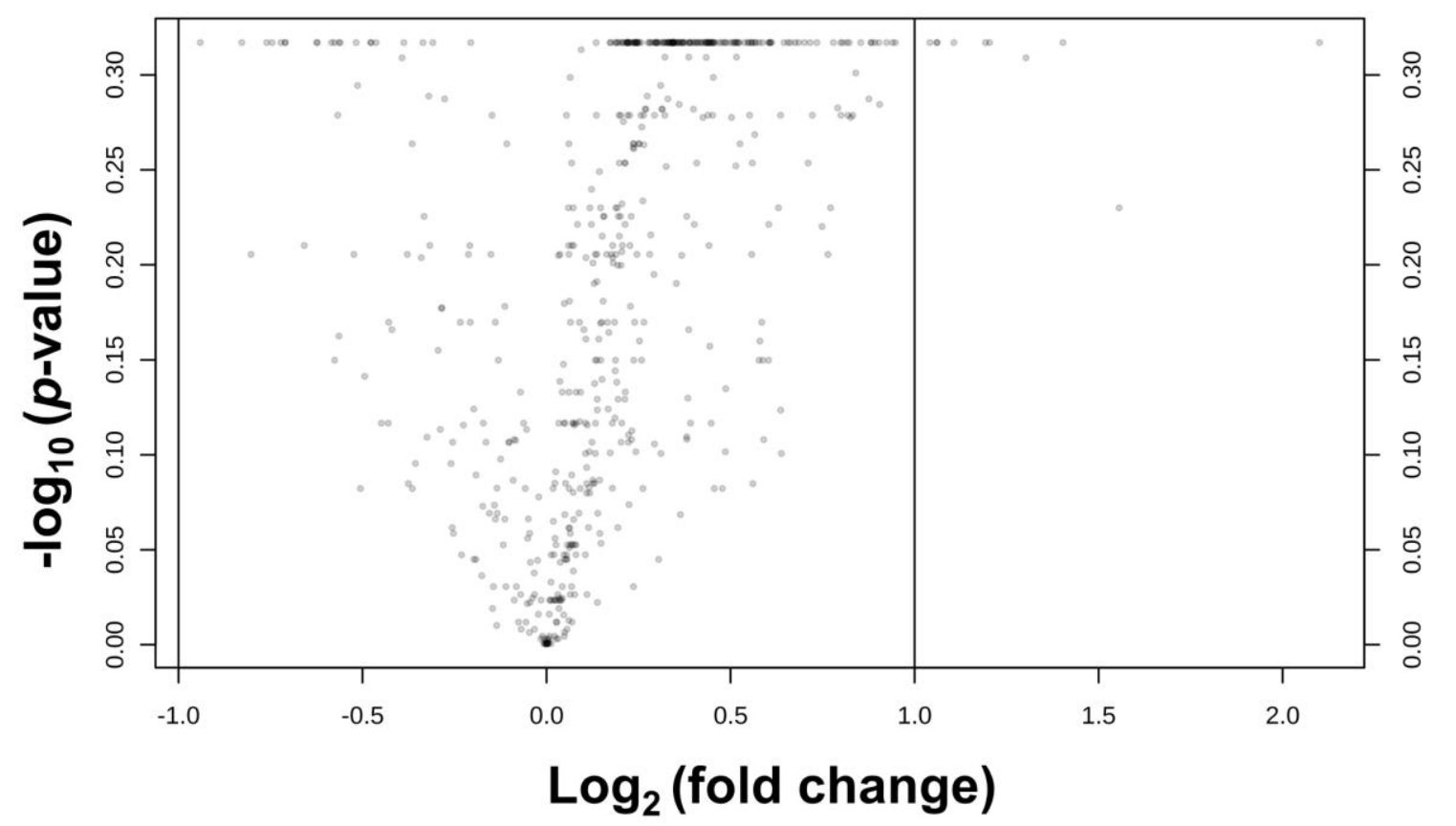

Supplementary Figure S4. Volcano plot of (A) raw broccoli samples and (B) steamed broccoli samples. Fold change was calculated by peak intensity of control broccoli over peak intensity of MeJA-treated broccoli. 
(A)

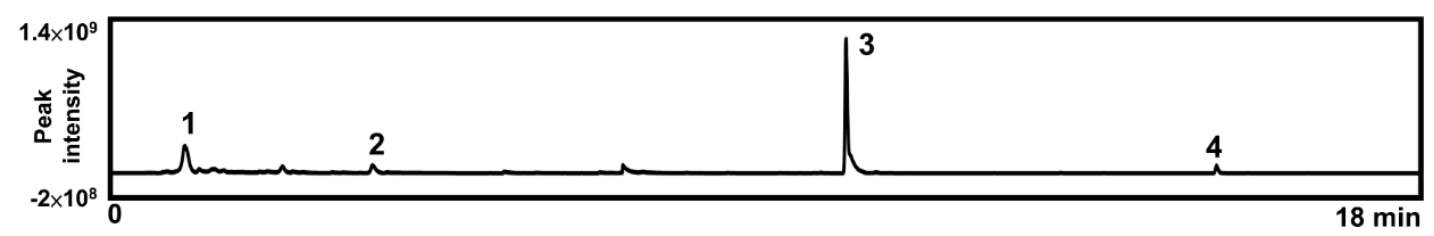

(B)

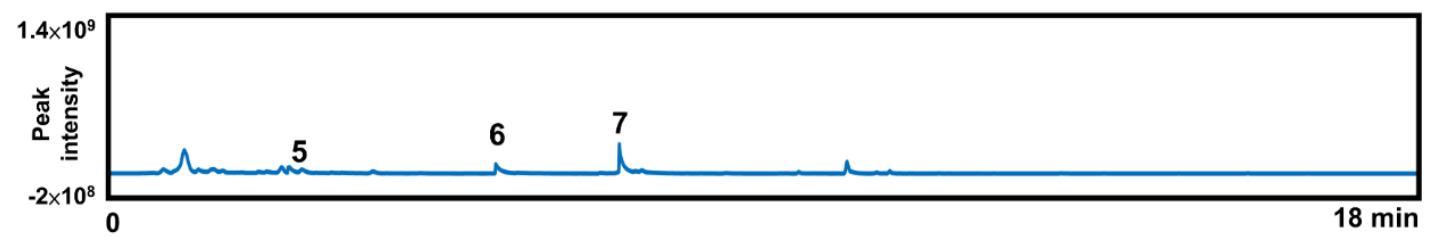

(C)

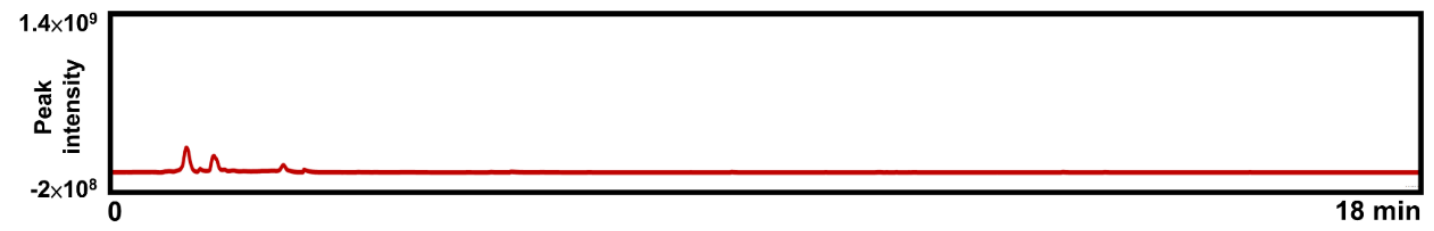

(D)

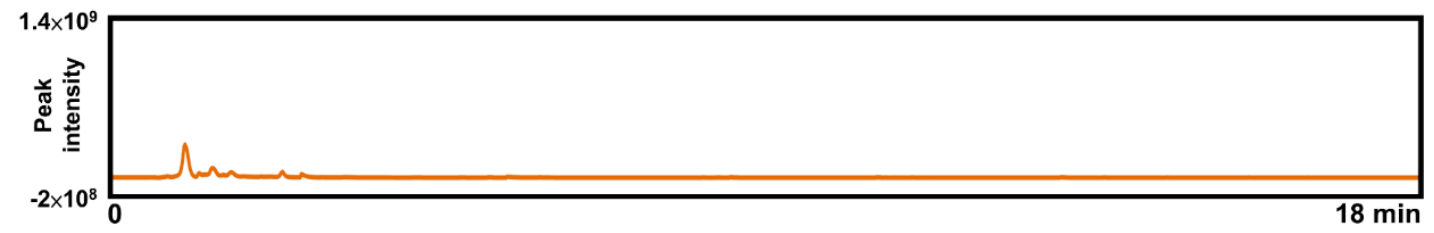

Supplementary Figure S5 GC chromatograph of volatile profile from control and MeJA-treated broccoli using ITEX. (A) Raw control broccoli, (B) Raw MeJA-treated broccoli, (C) Steamed control broccoli, and (D) Steamed MeJA-treated broccoli.

The major volatile compound in the graph: (1) dimethyl sulfide, (2) dimethyl disulfide, (3) dimethyl trisulfide, (4) dimethyl tetrasulfide, (5) 2-ethylfuran, (6) hexanal, and (7) (E)-2-hexanal. 
(A)

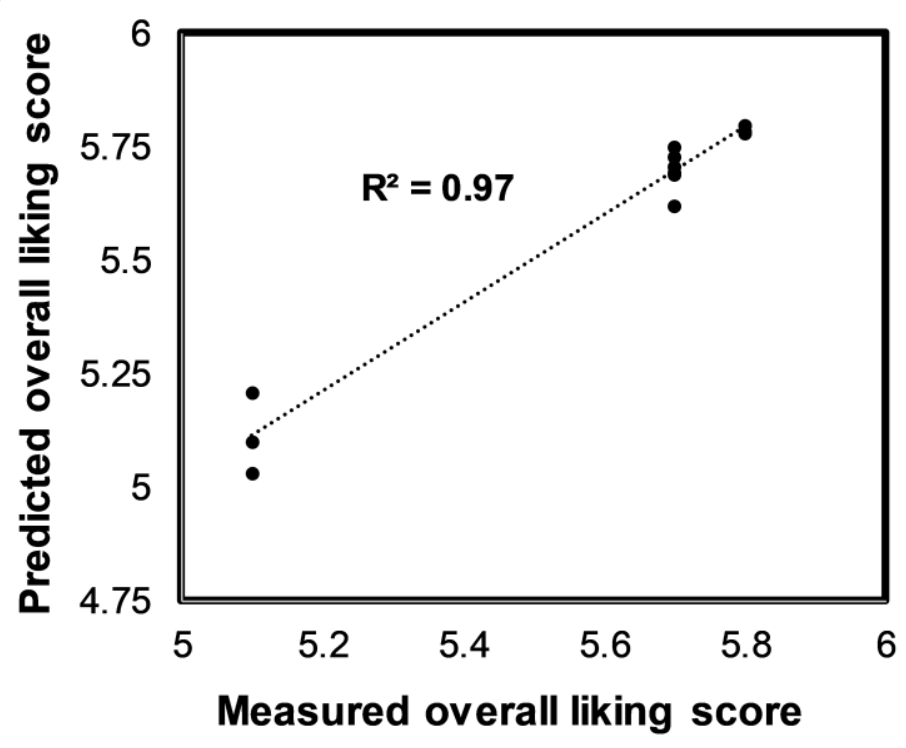

(B)

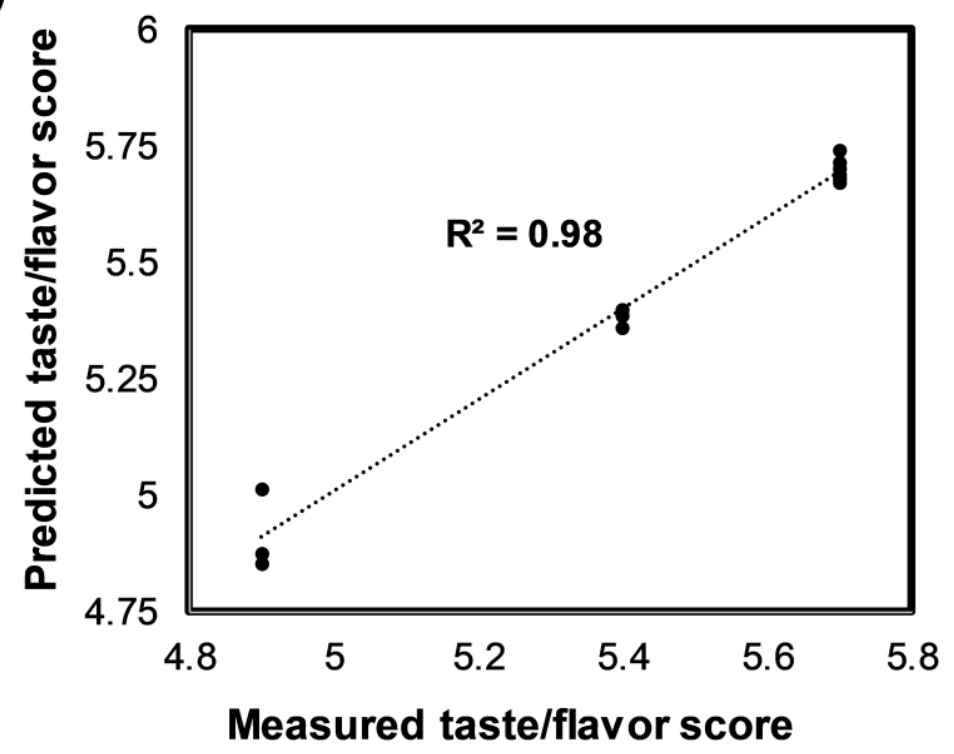

Supplementary Figure S6. Partial least square -regression prediction model of (A) overall liking score and (B) taste/favor score based on 57 measured metabolites including primary metabolites, glucosinolates, glucosinolate hydrolysis products, and volatiles. 


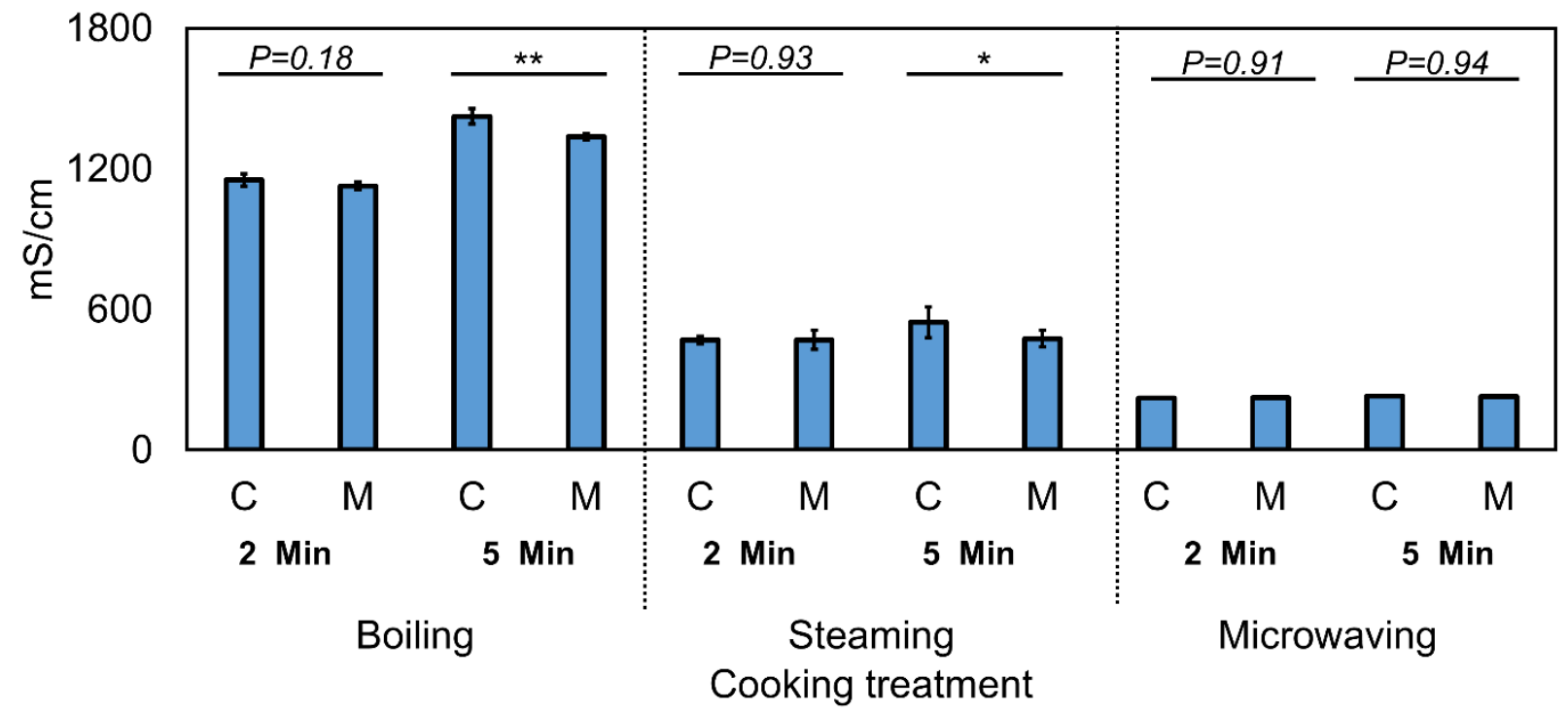

Supplementary Figure S7 Electrical conductivity in cooking water. Asterisk (*) indicates significant difference with or without MeJA within the same cooking treatment by Student's Ttest $(P \leq 0.05, N=3)^{\mathrm{ns}}, *, P \leq 0.05 ; * *, P \leq 0.01 ; * * *, P \leq 0.001$. 
Supplementary Document 1. IRB Approval Letter for Sensory Evaluation

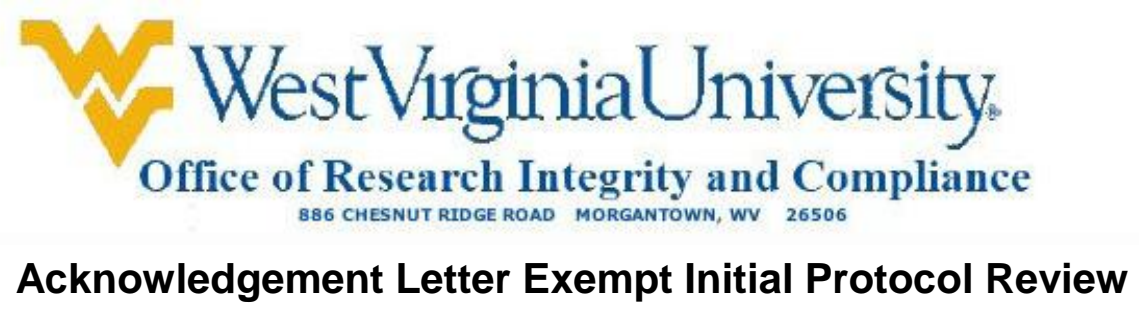
Action Date
$05 / 13 / 2016$
To
Kristen Matak
From
WVU Office of Research Integrity and Compliance
Approval Date
$05 / 13 / 2016$
Expiration Date
$05 / 12 / 2019$
Subject
Acknowledgement Letter Exempt Initial Protocol Review
Protocol Number
1605114506
Title
Pre-harvest methyl jasmonate treatment on the sensory evaluation and consumer acceptance of broccoli

The above-referenced study was reviewed by the West Virginia University Institutional Review Board IRB and was granted exemption in accordance with 45 CFR 46.101.

- This research study was granted an exemption because the Research involves educational tests, survey procedures, interview procedures or observation of public behavior and (i) information obtained is recorded in such a manner that human subjects cannot be identified, directly or through identifiers linked to the subjects; and (ii) any disclosure of the human subjects responses outside the research could not reasonably place the subjects at risk of criminal or civil liability or be damaging to the subjects financial standing, employability, or reputation [45 CFR 46.101(2)]. All exemptions are only good for three years. If this research extends more than three years beyond the approved date, then the researcher will have to request another exemption. The following documents have been acknowledged for use in this study and are available in the WVU+kc system:

Documents reviewed and/or approved as part of this submission:

Recruitment email.docx: 2016-05-12-04:00

Broccoli Flyer.pptx: 2016-05-12-04:00

Human Subjects Form Broccoli.doc: 2016-05-12-04 
Documents for use in this study have been acknowledged and are available in the WVUkc system in the Notes and Attachments section of your protocol.

The Office of Research Integrity and Compliance is here to provide assistance to you from the initial submission of an IRB protocol and all subsequent activity. Please feel free to contact us by phone at 304.293.7073 with any question you may have. Thank you.

WVU Office of Research Integrity and

Compliance Date:05/13/2016

Signed:

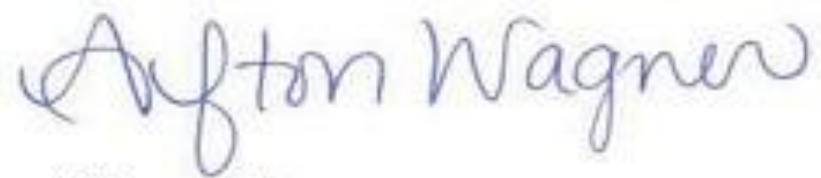

Afton Wagner

IRB Administrator 
Supplementary Document 2. Consumer Survey Questionnaire

Title of Project Sensory Evaluation of Nutritionally Enhanced Broccoli

1. What is your gender? Male Female

2. What is your age?
a. $18-35$ years
b. $36-54$ years
c. over 55 years

3. Do you think what people eat are related to health problems like high blood pressure or cancer? Yes or No

4. Do you like fruits and vegetables? Yes or No

5. Do you like broccoli? Yes or No

What is the main reason for your response? (Please choose one)
a. Flavor
b. Nutritional benefits (fiber, vitamins, etc.)
c. Visual appearance

Other reasons

6. Which do you buy more often (circle): fresh or frozen broccoli

7. How often do you consume broccoli?
a. daily
b. weekly
c. monthly
d. never

8. Do you purchase fresh broccoli? Yes or No If you said yes, what is the most important trait if you purchase broccoli? (Please choose one)
a. Visual color (greenness)
b. Uniformity of floret
c. Price
d. Certified organic
e. Locally grown

Other reasons

9. How do you typically eat broccoli?
a. Raw
b. Boiled 

c. Steamed
d. Microwaved
e. Fried
f. Roasted
g. Other

10. When you eat broccoli, which are the main nutritional components you expect to gain? (please choose one)
a. Vitamins \& minerals
b. Anticancer compounds
c. Antioxidant compounds
d. Fiber
e. Other

11. Which of the following traits would be most attractive to you? (please choose one)
a. Better tasting broccoli
b. Anticancer compounds
c. Longer shelf-life broccoli
d. Better looking (greener, brighter) broccoli
e. Smaller floret size

12. Would you be willing to pay more for broccoli that has twice the amount of cancer fighting compounds? Yes or No

How much more would you pay? (Select maximum price you are willing to pay for it).
a. No more
b. $\$ 0.25 /$ head
c. $\$ 0.50 /$ head
d. $\$ 0.75 /$ head
e. $\$ 1.00 /$ head
f. $\$ 1.50 /$ head 
Supplementary Document 3. Focus Group Questionnaire for local growers

1. Name of participant

2. Gender and age

3. How long you have been growing vegetables? years

4. Location of farm (Town, State)

5. Which category does your farm fall under (circle one)

$$
\text { Conventional Farm or Organic Farm }
$$

6. What is the size of your farm (in acres)?

You will see three parts of the questionnaires. Please fill in the first part. The questions present in the second and third part are for focus group discussion.

Part I: Production scale and budget (cultivation area, harvest yield, and types of vegetables produced)

1. How many acres of broccoli and total vegetables do you currently grow?

Broccoli Total vegetables

2. Broccoli cultivar used

3. On this area, what types of vegetables do you grow in addition to broccoli? List 3 major ones.

a.

b. 
c.

4. How much (in weight) can you harvest for the vegetables mentioned above year-round?

Broccoli

a.

b.

c.

5. Do you use pesticides? If so, what types of pesticides do you use for the vegetables and how often do you apply the pesticides?

(Circle one) Yes No

Name of Pesticides

Active ingredient

6. How much does it cost you to grow broccoli and some major vegetables in the growing season (In USD per acre)? What is the cost for the entire farm? (In USD per acre)

\begin{tabular}{|c|l|l|l|l|l|l|}
\hline & Broccoli & & & & & $\begin{array}{c}\text { Farm } \\
\text { total }\end{array}$ \\
\hline Seed & & & & & & \\
\hline Labor & & & & & & \\
\hline Utility & & & & & & \\
\hline $\begin{array}{c}\text { Machine } \\
\text { maintenance }\end{array}$ & & & & & & \\
\hline $\begin{array}{c}\text { Transportation } \\
\text { Tax }\end{array}$ & & & & & & \\
\hline Pesticide & & & & & & \\
\hline Fertilizer & & & & & & \\
\hline Other item 1 & & & & & \\
\hline Name & & & & & & \\
\hline
\end{tabular}




\begin{tabular}{|c|l|l|l|l|l|l|}
\hline Amount & \multicolumn{2}{|l|}{} & & & & \\
\hline Other item 2 & & & & & & \\
\hline Name & & & & & & \\
\hline Amount & & & & & & \\
\hline Other item 3 & \multicolumn{2}{|l|}{} & & & \\
\hline Name & & & & & & \\
\hline Amount & & & & & & \\
\hline Total cost & & & & & & \\
\hline
\end{tabular}

7. What is the sale price of broccoli and major vegetables? What are the revenue and gross income?

\begin{tabular}{|l|l|l|l|}
\hline Vegetable & Sale price (\$ per lb.) & Revenue & Gross income \\
\hline Broccoli & & & \\
\hline & & & \\
\hline & & & \\
\hline & & & \\
\hline All vegetables & & & \\
\hline
\end{tabular}

8. What is the most demanding part in your current production?

\section{Part II: Discussion on adopting a new practice (MeJA application) in the production}

In this section, the moderator will introduce the background information about methyl jasmonate and how it can increase the health benefits of broccoli. The following questions will be discussed with local farmers after the introduction.

1. Current optimized application method is to apply $250 \mu \mathrm{M}$ MeJA 4-day prior to harvest. Can you think of any potential disadvantages?

2. What will be the most concerning point for you about this application? Is this practical to you? What are the major reasons for your answer? 
3. What kind of proof or information will you want to see from the research group to convince you to adopt this practice?

4. If this new practice is not feasible on broccoli, can you think of alternative crop that we can use?

5. By your experience do you think the customers will accept this new practice?

\section{Part III: Challenges in broccoli or Brassica vegetable production}

1. Please describe the type of markets you are in. At which locations are these markets? Currently, which market brings you the most revenue?

2. What are the main/current obstacles you face when producing broccoli or Brassica vegetable? Have you tried to resolve them? How?

3. Have you ever raised the selling price for broccoli or any other vegetables? Why? How did customers react to the price increase? 
Supplementary Document 4. IRB Focus Group Approval Letter

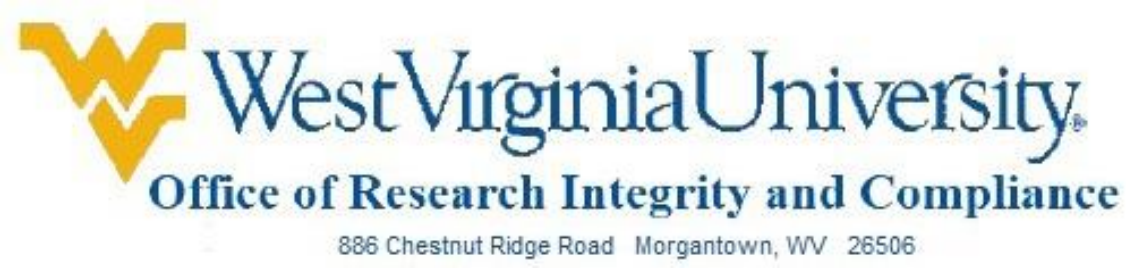

\section{Acknowledgement of Exemption}

$08 / 09 / 2018$

To: Xiaoli Etienne

From: WVU Office of Research Integrity \& Compliance

Protocol Type: Exempt

Submission Type: Initial

Funding: N/A
Approval Date: 08/09/2018

Expiration Date: 08/08/2021

WVU Protocol \#: 1806147205

Protocol Title: Focus group discussion of using methyl jasmonate application on broccoli production

The West Virginia University Institutional Review Board has reviewed your submission of

Exempt protocol 1806147205. Additional details regarding the review are below:

- This research study was granted an exemption because the Research involves educational tests, survey procedures, interview procedures or observation of public behavior and (i) information obtained is recorded in such a manner that human subjects cannot be identified, directly or through identifiers linked to the subjects; and (ii) any disclosure of the human subjects responses outside the research could not reasonably place the subjects at risk of criminal or civil liability or be damaging to the subjects financial standing, employability, or reputation [45 CFR 46.101(2)]. All exemptions are only good for three years. If this research extends more than three years beyond the approved date, then the researcher will have to request another exemption. The following documents have been acknowledged for use in this study and are available in the WVU+kc system:

The following documents were reviewed and approved for use as part of this submission. Only the documents listed below may be used in the research. Please access and print the files in the Notes \& 
Attachments section of your approved protocol.

- Permission Letter - Yu-chun - Broccoli Focus Group.pdf

- Local Farmer questionaire_Etilenne.docx

- Cover Letter_focus group.docx

- Video recording consent form.pdf

\section{WVU IRB acknowledgement of protocol 1806147205 will expire on 08/08/2021.}

If the study is to continue beyond the expiration date, a renewal application must be submitted no later than two (2) weeks prior to expiration date. It is your responsibility to submit your protocol for renewal.

Once you begin your human subjects research, the following regulations apply:

1. Unanticipated or serious adverse events and/or side effects encountered in this research study must be reported to the IRB within five (5) days, using the Notify IRB action in the electronic protocol.

2. Any modifications to the study protocol should be submitted only if there will be an increase in risk to subjects accompanying the proposed change(s).

3. You may not use a modified information sheet until it has been reviewed and acknowledged by the WVU IRB prior to implementation.

The Office of Research Integrity and Compliance will be glad to provide assistance to you throughout the research process. Please feel free to contact us by phone, at 304.293 .7073 or by email at IRB@mail.wvu.edu.

Sincerely,

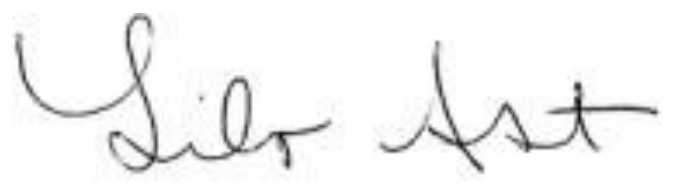

Lile Ast

IRB Administrator 
Supplementary Document 5. Video Recording \& Transcription Consent Form

West VirginiaUniversity。
DAVIS COLLEGE OF AGRICULTURE,
NATURAL RESOURCES AND DESIGN

\section{VIDEO RECORDING \& TRANSCRIPTION CONSENT FORM}

Focus group discussion of using methyl jasmonate application on broccoli production

Yu-Chun Chiu, Division of Plant and Soil Sciences

Dr. Xiaoli L. Etienne, Division of Resource Economics and Management

Dr. Kang-Mo Cu, Division of Plant and Soil Sciences

This study involves the video recording of your interview with the researcher. Neither your name nor any other identifying information will be associated with the video recording or the transcript. Only the research team will be able to listen (view) to the recordings. The tapes will be transcribed by the researcher and erased once the transcriptions are checked for accuracy. Transcripts of your interview may be reproduced in whole or in part for use in presentations or written products that result from this study. Neither your name nor any other identifying information (such as your voice or picture) will be used in presentations or in written products resulting from the study.

By signing this form, I am allowing the researcher to video tape me as part of this research.

Participant's Signature:

Date: 


\section{Reference list}

Able, A. J., Wong, L. S., Prasad, A., \& O'Hare, T. J. (2002). 1-MCP is more effective on a floral brassica (Brassica oleracea var. italica L.) than a leafy brassica (Brassica rapa var. chinensis). Postharvest Biology and technology, 26(2), 147-155.

Ahmad, P., Rasool, S., Gul, A., Sheikh, S. A., Akram, N. A., Ashraf, M., ... Gucel, S. (2016). Jasmonates: Multifunctional Roles in Stress Tolerance. Frontiers in Plant Science, 7. https://doi.org/10.3389/fpls.2016.00813

Ambrosone, C. B., McCann, S. E., Freudenheim, J. L., Marshall, J. R., Zhang, Y., \& Shields, P. G. (2004). Breast Cancer Risk in Premenopausal Women Is Inversely Associated with Consumption of Broccoli, a Source of Isothiocyanates, but Is Not Modified by GST Genotype. The Journal of Nutrition, 134(5), 1134-1138.

Andreyeva, T., Long, M. W., \& Brownell, K. D. (2010a). The Impact of Food Prices on Consumption: A Systematic Review of Research on the Price Elasticity of Demand for Food. American Journal of Public Health, 100(2), 216-222. https://doi.org/10.2105/AJPH.2008.151415

Andreyeva, T., Long, M. W., \& Brownell, K. D. (2010b). The Impact of Food Prices on Consumption: A Systematic Review of Research on the Price Elasticity of Demand for Food. American Journal of Public Health, 100(2), 216-222. https://doi.org/10.2105/AJPH.2008.151415

Ashraf, M., \& McNeilly, T. (2004). Salinity Tolerance in Brassica Oilseeds. Critical Reviews in Plant Sciences, 23(2), 157-174. https://doi.org/10.1080/07352680490433286

Atwell, L. L., Hsu, A., Wong, C. P., Stevens, J. F., Bella, D., Yu, T.-W., ... Ho, E. (2015). Absorption and chemopreventive targets of sulforaphane in humans following 
consumption of broccoli sprouts or a myrosinase-treated broccoli sprout extract.

Molecular Nutrition \& Food Research, 59(3), 424-433.

https://doi.org/10.1002/mnfr.201400674

Auborn, K. J., Fan, S., Rosen, E. M., Goodwin, L., Chandraskaren, A., Williams, D. E., ...

Carter, T. H. (2003). Indole-3-Carbinol Is a Negative Regulator of Estrogen. The Journal of Nutrition, 133(7), 2470S-2475S.

Augustine, R., Majee, M., Gershenzon, J., \& Bisht, N. C. (2013). Four genes encoding MYB28, a major transcriptional regulator of the aliphatic glucosinolate pathway, are differentially expressed in the allopolyploid Brassica juncea. Journal of Experimental Botany, 64(16), 4907-4921. https://doi.org/10.1093/jxb/ert280

Badenes-Perez, F. R., Gershenzon, J., \& Heckel, D. G. (2014). Insect Attraction versus Plant Defense: Young Leaves High in Glucosinolates Stimulate Oviposition by a Specialist Herbivore despite Poor Larval Survival due to High Saponin Content. PLoS ONE, 9(4), e95766. https://doi.org/10.1371/journal.pone.0095766

Baggaley, A. R. (1983). Deciding on the ratio of number of subjects to number of variables in factor analysis. Retrieved March 16, 2018, from http://psycnet.apa.org.www.libproxy.wvu.edu/record/1983-31730-001

Bailey, N. E., Arnold, S. K., \& Igo, C. G. (2014). Educating the Future of Agriculture: A Focus Group Analysis of the Programming Needs and Preferences of Montana Young and Beginning Farmers and Ranchers. Journal of Agricultural Education, 55(2), 167-183. https://doi.org/10.5032/jae.2014.02167 
Barak, J. D., Liang, A., \& Narm, K.-E. (2008). Differential Attachment to and Subsequent Contamination of Agricultural Crops by Salmonella enterica. Applied and Environmental Microbiology, 74(17), 5568-5570. https://doi.org/10.1128/AEM.01077-08

Beck, T. K., Jensen, S., Bjoern, G. K., \& Kidmose, U. (2014). The Masking Effect of Sucrose on Perception of Bitter Compounds in Brassica Vegetables. Journal of Sensory Studies, 29(3), 190-200. https://doi.org/10.1111/joss.12094

Becker, T. M., \& Juvik, J. A. (2016). The Role of Glucosinolate Hydrolysis Products from Brassica Vegetable Consumption in Inducing Antioxidant Activity and Reducing Cancer Incidence. Diseases, 4(2), 22. https://doi.org/10.3390/diseases4020022

Beekwilder, J., Leeuwen, W. van, Dam, N. M. van, Bertossi, M., Grandi, V., Mizzi, L., ... Bovy, A. (2008). The Impact of the Absence of Aliphatic Glucosinolates on Insect Herbivory in Arabidopsis. PLOS ONE, 3(4), e2068. https://doi.org/10.1371/journal.pone.0002068

Bell, L., Methven, L., Signore, A., Oruna-Concha, M. J., \& Wagstaff, C. (2017). Analysis of seven salad rocket (Eruca sativa) accessions: The relationships between sensory attributes and volatile and non-volatile compounds. Food Chemistry, 218, 181-191. https://doi.org/10.1016/j.foodchem.2016.09.076

Bell, L., Methven, L., \& Wagstaff, C. (2017). The influence of phytochemical composition and resulting sensory attributes on preference for salad rocket (Eruca sativa) accessions by consumers of varying TAS2R38 diplotype. Food Chemistry, 222, 6-17. https://doi.org/10.1016/j.foodchem.2016.11.153

Bell, L., Oloyede, O. O., Lignou, S., Wagstaff, C., \& Methven, L. (2018). Taste and Flavor Perceptions of Glucosinolates, Isothiocyanates, and Related Compounds. Molecular Nutrition \& Food Research, 62(18), 1700990. https://doi.org/10.1002/mnfr.201700990 
Bell, L., \& Wagstaff, C. (2017). Enhancement Of Glucosinolate and Isothiocyanate Profiles in Brassicaceae Crops: Addressing Challenges in Breeding for Cultivation, Storage, and Consumer-Related Traits. Journal of Agricultural and Food Chemistry, 65(43), 93799403. https://doi.org/10.1021/acs.jafc.7b03628

Benevenuto, R. F., Seldal, T., Hegland, S. J., Rodriguez-Saona, C., Kawash, J., \& Polashock, J. (2019). Transcriptional profiling of methyl jasmonate-induced defense responses in bilberry (Vaccinium myrtillus L.). BMC Plant Biology, 19. https://doi.org/10.1186/s12870-019-1650-0

Benson, A. M., Hunkeler, M. J., \& Talalay, P. (1980). Increase of NAD(P)H:quinone reductase by dietary antioxidants: possible role in protection against carcinogenesis and toxicity. Proceedings of the National Academy of Sciences, 77(9), 5216-5220. https://doi.org/10.1073/pnas.77.9.5216

Bernardi, R., Negri, A., Ronchi, S., \& Palmieri, S. (2000). Isolation of the epithiospecifier protein from oil-rape (Brassica napus ssp. oleifera) seed and its characterization. FEBS Letters, 467(2-3), 296-298. https://doi.org/10.1016/S0014-5793(00)01179-0

Bidart-Bouzat, M. G., \& Kliebenstein, D. (2011). An ecological genomic approach challenging the paradigm of differential plant responses to specialist versus generalist insect herbivores. Oecologia, 167(3), 677. https://doi.org/10.1007/s00442-011-2015-z

Biondi, S., Scaramagli, S., Capitani, F., Maddalena Altamura, M., \& Torrigiani, P. (2001). Methyl jasmonate upregulates biosynthetic gene expression, oxidation and conjugation of polyamines, and inhibits shoot formation in tobacco thin layers. Journal of Experimental Botany, 52(355), 231-242. https://doi.org/10.1093/jexbot/52.355.231 
Björkman, M., Klingen, I., Birch, A. N. E., Bones, A. M., Bruce, T. J. A., Johansen, T. J., ... Stewart, D. (2011). Phytochemicals of Brassicaceae in plant protection and human health - Influences of climate, environment and agronomic practice. Phytochemistry, 72(7), 538-556. https://doi.org/10.1016/j.phytochem.2011.01.014

Björkman, R., \& Lönnerdal, B. (1973). Studies on myrosinases III. Enzymatic properties of myrosinases from Sinapis alba and Brassica napus seeds. Biochimica et Biophysica Acta (BBA) - Enzymology, 327(1), 121-131. https://doi.org/10.1016/0005-2744(73)90109-5

Björkman, T., \& Pearson, K. J. (1998). High temperature arrest of inflorescence development in broccoli (Brassica oleracea var. italica L.). Journal of Experimental Botany, 49(318), 101-106. Retrieved from JSTOR.

Bongoni, R., Verkerk, R., Steenbekkers, B., Dekker, M., \& Stieger, M. (2014). Evaluation of Different Cooking Conditions on Broccoli (Brassica oleracea var. italica) to Improve the Nutritional Value and Consumer Acceptance. Plant Foods for Human Nutrition, 69(3), 228-234. https://doi.org/10.1007/s11130-014-0420-2

Boonyaritthongchai, P., Chimvaree, C., Buanong, M., Uthairatanakij, A., \& Jitareerat, P. (2016). Effect of Methyl Jasmonate on Physical and Chemical Properties of Mango Fruit cv. Nam Dok Mai. Horticulturae, 3(1), 18. https://doi.org/10.3390/horticulturae3010018

Bozorov, T. A., Dinh, S. T., \& Baldwin, I. T. (2017). JA but not JA-Ile is the cellnonautonomous signal activating JA mediated systemic defenses to herbivory in Nicotiana attenuata. Journal of Integrative Plant Biology, 59(8), 552-571. https://doi.org/10.1111/jipb.12545

Brown, A. F., Yousef, G. G., Jeffery, E. H., Klein, B. P., Wallig, M. A., Kushad, M. M., \& Juvik, J. A. (2002). Glucosinolate Profiles in Broccoli: Variation in Levels and Implications in 
Breeding for Cancer Chemoprotection. Journal of the American Society for Horticultural Science, 127(5), 807-813.

Bruinsma, M., Van Dam, N. M., Van Loon, J. J. A., \& Dicke, M. (2007). Jasmonic Acid-Induced Changes in Brassica oleracea Affect Oviposition Preference of Two Specialist Herbivores. Journal of Chemical Ecology, 33(4), 655-668. https://doi.org/10.1007/s10886-006-9245-2

Burow, M., Markert, J., Gershenzon, J., \& Wittstock, U. (2006). Comparative biochemical characterization of nitrile-forming proteins from plants and insects that alter myrosinasecatalysed hydrolysis of glucosinolates. FEBS Journal, 273(11), 2432-2446. https://doi.org/10.1111/j.1742-4658.2006.05252.x

Campbell, M. K., Demark-Wahnefried, W., Symons, M., Kalsbeek, W. D., Dodds, J., Cowan, A., ... McClelland, J. W. (1999). Fruit and vegetable consumption and prevention of cancer: the Black Churches United for Better Health project. American Journal of Public Health, 89(9), 1390-1396. https://doi.org/10.2105/AJPH.89.9.1390

Campos, M. L., Yoshida, Y., Major, I. T., Ferreira, D. de O., Weraduwage, S. M., Froehlich, J. E., ... Howe, G. A. (2016). Rewiring of jasmonate and phytochrome B signalling uncouples plant growth-defense tradeoffs. Nature Communications, 7, 12570. https://doi.org/10.1038/ncomms12570

Cao, J., Li, M., Chen, J., Liu, P., \& Li, Z. (2016). Effects of MeJA on Arabidopsis metabolome under endogenous JA deficiency. Scientific Reports, 6, srep37674. https://doi.org/10.1038/srep37674 
Cao, S., Zheng, Y., Wang, K., Rui, H., \& Tang, S. (2010). Effect of methyl jasmonate on cell wall modification of loquat fruit in relation to chilling injury after harvest. Food Chemistry, 118(3), 641-647. https://doi.org/10.1016/j.foodchem.2009.05.047

Capriotti, A. L., Cavaliere, C., La Barbera, G., Montone, C. M., Piovesana, S., Zenezini Chiozzi, R., \& Laganà, A. (2018). Chromatographic column evaluation for the untargeted profiling of glucosinolates in cauliflower by means of ultra-high performance liquid chromatography coupled to high resolution mass spectrometry. Talanta, 179, 792-802. https://doi.org/10.1016/j.talanta.2017.12.019

Cartea, M. E., Francisco, M., Soengas, P., \& Velasco, P. (2011). Phenolic Compounds in Brassica Vegetables. Molecules, 16(1), 251-280. https://doi.org/10.3390/molecules 16010251

Chadwick, M., Gawthrop, F., Michelmore, R. W., Wagstaff, C., \& Methven, L. (2016). Perception of bitterness, sweetness and liking of different genotypes of lettuce. Food Chemistry, 197, 66-74. https://doi.org/10.1016/j.foodchem.2015.10.105

Chehab, E. W., Kaspi, R., Savchenko, T., Rowe, H., Negre-Zakharov, F., Kliebenstein, D., \& Dehesh, K. (2008). Distinct Roles of Jasmonates and Aldehydes in Plant-Defense Responses. PLOS ONE, 3(4), e1904. https://doi.org/10.1371/journal.pone.0001904

Chen, Y., Fei, M., Wang, Y., Chen, S., \& Yan, X. (2015). Proteomic investigation of glucosinolate systematically changes in Arabidopsis Rosette leaves to exogenous methyl jasmonate. Plant Biosystems - An International Journal Dealing with All Aspects of Plant Biology, 149(2), 346-353. https://doi.org/10.1080/11263504.2013.819044

Chen, Y.-J., Wallig, M. A., \& Jeffery, E. H. (2016). Dietary Broccoli Lessens Development of Fatty Liver and Liver Cancer in Mice Given Diethylnitrosamine and Fed a Western or 
Control Diet. The Journal of Nutrition, 146(3), 542-550.

https://doi.org/10.3945/jn.115.228148

Cheong, J.-J., \& Choi, Y. D. (2003). Methyl jasmonate as a vital substance in plants. Trends in Genetics, 19(7), 409-413. https://doi.org/10.1016/S0168-9525(03)00138-0

Chikkamath, M., Atteri, B., Srivastava, S. K., \& Roy, S. (2012). Factors influencing consumers behaviour for vegetable purchase. 39(1), 6 .

Chiu, Y.-C., Juvik, J. A., \& Ku, K.-M. (2018). Targeted Metabolomic and Transcriptomic Analyses of "Red Russian” Kale (Brassicae napus var. pabularia) Following Methyl Jasmonate Treatment and Larval Infestation by the Cabbage Looper (Trichoplusia ni Hübner). International Journal of Molecular Sciences, 19(4), 1058. https://doi.org/10.3390/ijms19041058

Chiu, Y.-C., Matak, K., \& Ku, K.-M. (2019). Methyl jasmonate treated broccoli: Impact on the production of glucosinolates and consumer preferences. Food Chemistry, 299, 125099. https://doi.org/10.1016/j.foodchem.2019.125099

Chong, J., Soufan, O., Li, C., Caraus, I., Li, S., Bourque, G., ... Xia, J. (2018). MetaboAnalyst 4.0: towards more transparent and integrative metabolomics analysis. Nucleic Acids Research, 46(W1), W486-W494. https://doi.org/10.1093/nar/gky310

Clarke, D. B. (2010a). Glucosinolates, structures and analysis in food. Analytical Methods, 2(4), 310-325. https://doi.org/10.1039/B9AY00280D

Clarke, D. B. (2010b). Glucosinolates, structures and analysis in food. Analytical Methods, 2(4), 310-325. https://doi.org/10.1039/B9AY00280D 
Costell, E., Tárrega, A., \& Bayarri, S. (2010). Food Acceptance: The Role of Consumer Perception and Attitudes. Chemosensory Perception, 3(1), 42-50. https://doi.org/10.1007/s12078-009-9057-1

Cowart, B. J., Yokomukai, Y., \& Beauchamp, G. K. (1994). Bitter taste in aging: Compoundspecific decline in sensitivity. Physiology \& Behavior, 56(6), 1237-1241. https://doi.org/10.1016/0031-9384(94)90371-9

Cox, D. N., Melo, L., Zabaras, D., \& Delahunty, C. M. (2012). Acceptance of health-promoting Brassica vegetables: the influence of taste perception, information and attitudes. Public Health Nutrition, 15(08), 1474-1482. https://doi.org/10.1017/S1368980011003442

del Carmen Martínez-Ballesta, M., Moreno, D. A., \& Carvajal, M. (2013). The Physiological Importance of Glucosinolates on Plant Response to Abiotic Stress in Brassica. International Journal of Molecular Sciences, 14(6), 11607-11625. https://doi.org/10.3390/ijms140611607

Demole, E., Lederer, E., \& Mercier, D. (1962). Isolement et détermination de la structure du jasmonate de méthyle, constituant odorant caractéristique de l'essence de jasmin. Helvetica Chimica Acta, 45(2), 675-685. https://doi.org/10.1002/hlca.19620450233

Devoto, A., Ellis, C., Magusin, A., Chang, H.-S., Chilcott, C., Zhu, T., \& Turner, J. G. (2005). Expression profiling reveals COI1 to be a key regulator of genes involved in wound- and methyl jasmonate-induced secondary metabolism, defence, and hormone interactions. Plant Molecular Biology, 58(4), 497-513. https://doi.org/10.1007/s11103-005-7306-5 Dombrecht, B., Xue, G. P., Sprague, S. J., Kirkegaard, J. A., Ross, J. J., Reid, J. B., ... Kazan, K. (2007). MYC2 Differentially Modulates Diverse Jasmonate-Dependent Functions in 
Arabidopsis. The Plant Cell Online, 19(7), 2225-2245.

https://doi.org/10.1105/tpc.106.048017

Doorn, H. E. van, Kruk, G. C. van der, Holst, G.-J. van, Raaijmakers-Ruijs, N. C. M. E., Postma, E., Groeneweg, B., \& Jongen, W. H. F. (1998). The glucosinolates sinigrin and progoitrin are important determinants for taste preference and bitterness of Brussels sprouts. Journal of the Science of Food and Agriculture, 78(1), 30-38. https://doi.org/10.1002/(SICI)1097-0010(199809)78:1<30::AID-JSFA79>3.0.CO;2-N

Dosz, E. B., Ku, K.-M., Juvik, J. A., \& Jeffery, E. H. (2014). Total myrosinase activity estimates in brassica vegetable produce. Journal of Agricultural and Food Chemistry, 62(32), 8094-8100. https://doi.org/10.1021/jf501692c

Drewnowski, A., \& Gomez-Carneros, C. (2000). Bitter taste, phytonutrients, and the consumer: a review. The American Journal of Clinical Nutrition, 72(6), 1424-1435. https://doi.org/10.1093/ajcn/72.6.1424

Ellis, C. (2002). The Arabidopsis Mutant cev1 Links Cell Wall Signaling to Jasmonate and Ethylene Responses. THE PLANT CELL ONLINE, 14(7), 1557-1566. https://doi.org/10.1105/tpc.002022

Engel, E., Baty, C., le Corre, D., Souchon, I., \& Martin, N. (2002a). Flavor-Active Compounds Potentially Implicated in Cooked Cauliflower Acceptance. Journal of Agricultural and Food Chemistry, 50(22), 6459-6467. https://doi.org/10.1021/jf025579u

Engel, E., Baty, C., le Corre, D., Souchon, I., \& Martin, N. (2002b). Flavor-Active Compounds Potentially Implicated in Cooked Cauliflower Acceptance. Journal of Agricultural and Food Chemistry, 50(22), 6459-6467. https://doi.org/10.1021/jf025579u 
Engel, E., Martin, N., \& Issanchou, S. (2006). Sensitivity to allyl isothiocyanate, dimethyl trisulfide, sinigrin, and cooked cauliflower consumption. Appetite, 46(3), 263-269. https://doi.org/10.1016/j.appet.2006.01.007

Fahey, J. W., Wehage, S. L., Holtzclaw, W. D., Kensler, T. W., Egner, P. A., Shapiro, T. A., \& Talalay, P. (2012). Protection of Humans by Plant Glucosinolates: Efficiency of Conversion of Glucosinolates to Isothiocyanates by the Gastrointestinal Microflora. Cancer Prevention Research, 5(4), 603-611. https://doi.org/10.1158/1940-6207.CAPR$11-0538$

Falk, K. L., Kästner, J., Bodenhausen, N., Schramm, K., Paetz, C., Vassão, D. G., ... Meldau, S. (2014). The role of glucosinolates and the jasmonic acid pathway in resistance of Arabidopsis thaliana against molluscan herbivores. Molecular Ecology, 23(5), 11881203. https://doi.org/10.1111/mec. 12610

FDA. (2013). Methyl Jasmonate; Exemption From the Requirement of a Tolerance. Retrieved from FDA-EPA website: https://www.federalregister.gov/documents/2013/04/17/201308829/methyl-jasmonate-exemption-from-the-requirement-of-a-tolerance

Fenwick, G. R., Griffiths, N. M., \& Heaney, R. K. (1983). Bitterness in brussels sprouts (Brassica oleracea L. var.gemmifera): The role of glucosinolates and their breakdown products. Journal of the Science of Food and Agriculture, 34(1), 73-80. https://doi.org/10.1002/jsfa.2740340111

Fernie, A. R., Carrari, F., \& Sweetlove, L. J. (2004). Respiratory metabolism: glycolysis, the TCA cycle and mitochondrial electron transport. Current Opinion in Plant Biology, 7(3), 254-261. https://doi.org/10.1016/j.pbi.2004.03.007 
Frerigmann, H., \& Gigolashvili, T. (2014). MYB34, MYB51, and MYB122 Distinctly Regulate Indolic Glucosinolate Biosynthesis in Arabidopsis thaliana. Molecular Plant, 7(5), 814828. https://doi.org/10.1093/mp/ssu004

Frier, J. P. D., Hernández, C. V. S., \& Tiessen, A. (2012). Friend or Foe? Exploring the Factors that Determine the Difference Between Positive and Negative Effects on Photosynthesis in Response to Insect Herbivory. https://doi.org/10.5772/26064

Frydoonfar, H. R., McGrath, D. R., \& Spigelman, A. D. (2003). The effect of indole-3-carbinol and sulforaphane on a prostate cancer cell line. ANZ Journal of Surgery, 73(3), 154-156.

Fuentes, F., Paredes-Gonzalez, X., \& Kong, A.-N. T. (2015). Dietary Glucosinolates Sulforaphane, Phenethyl Isothiocyanate, Indole-3-Carbinol/3,3'-Diindolylmethane: Antioxidative Stress/Inflammation, Nrf2, Epigenetics/Epigenomics and In Vivo Cancer Chemopreventive Efficacy. Current Pharmacology Reports, 1(3), 179-196. https://doi.org/10.1007/s40495-015-0017-y

Fürstenberg-Hägg, J., Zagrobelny, M., \& Bak, S. (2013). Plant Defense against Insect Herbivores. International Journal of Molecular Sciences, 14(5), 10242-10297. https://doi.org/10.3390/ijms 140510242

Garitta, L., Hough, G., \& Chaves, A. (2013). Sensory analysis of broccoli over time: Consumer defined critical attributes and evaluation of digital photographs in comparison to real product appearance. Food Quality and Preference, 29(1), 48-52. https://doi.org/10.1016/j.foodqual.2013.02.001

Gigolashvili, T., Berger, B., \& Flügge, U.-I. (2009). Specific and coordinated control of indolic and aliphatic glucosinolate biosynthesis by R2R3-MYB transcription factors in 
Arabidopsis thaliana. Phytochemistry Reviews, 8(1), 3-13. https://doi.org/10.1007/s11101-008-9112-6

Giskes, K., Van Lenthe, F. J., Brug, J., Mackenbach, J. P., \& Turrell, G. (2007). Socioeconomic inequalities in food purchasing: The contribution of respondent-perceived and actual (objectively measured) price and availability of foods. Preventive Medicine, 45(1), 4148. https://doi.org/10.1016/j.ypmed.2007.04.007

Greene, G. L. (1972). Economic Damage Threshold and Spray Interval for Cabbage Looper Control on Cabbage 1 2. Journal of Economic Entomology, 65(1), 205-208.

Hahn, C., Müller, A., Kuhnert, N., \& Albach, D. (2016). Diversity of Kale (Brassica oleracea var. sabellica): Glucosinolate Content and Phylogenetic Relationships. Journal of Agricultural and Food Chemistry, 64(16), 3215-3225. https://doi.org/10.1021/acs.jafc.6b01000

Halkier, B. A., \& Gershenzon, J. (2006). Biology and Biochemistry of Glucosinolates. Annual Review of Plant Biology, 57(1), 303-333. https://doi.org/10.1146/annurev.arplant.57.032905.105228

Hanaka, A., Maksymiec, W., \& Bednarek, W. (2015). The effect of methyl jasmonate on selected physiological parameters of copper-treated Phaseolus coccineus plants. Plant Growth Regulation, 77(2), 167-177. https://doi.org/10.1007/s10725-015-0048-8

Hanschen, F. S., Brüggemann, N., Brodehl, A., Mewis, I., Schreiner, M., Rohn, S., \& Kroh, L. W. (2012). Characterization of Products from the Reaction of Glucosinolate-Derived Isothiocyanates with Cysteine and Lysine Derivatives Formed in Either Model Systems or Broccoli Sprouts. Journal of Agricultural and Food Chemistry, 60(31), 7735-7745. https://doi.org/10.1021/jf301718g 
Hanschen, F. S., Kaufmann, M., Kupke, F., Hackl, T., Kroh, L. W., Rohn, S., \& Schreiner, M. (2018). Brassica vegetables as sources of epithionitriles: Novel secondary products formed during cooking. Food Chemistry, 245, 564-569. https://doi.org/10.1016/j.foodchem.2017.10.124

Hanschen, F. S., Kühn, C., Nickel, M., Rohn, S., \& Dekker, M. (2018). Leaching and degradation kinetics of glucosinolates during boiling of Brassica oleracea vegetables and the formation of their breakdown products. Food Chemistry, 263, 240-250. https://doi.org/10.1016/j.foodchem.2018.04.069

Hasperué, J. H., Gómez-Lobato, M. E., Chaves, A. R., Civello, P. M., \& Martínez, G. A. (2013). Time of day at harvest affects the expression of chlorophyll degrading genes during postharvest storage of broccoli. Postharvest Biology and Technology, 82, 22-27. https://doi.org/10.1016/j.postharvbio.2013.02.021

Hassini, I., Baenas, N., Moreno, D. A., Carvajal, M., Boughanmi, N., \& Ballesta, M. D. C. M. (2017). Effects of seed priming, salinity and methyl jasmonate treatment on bioactive composition of Brassica oleracea var. capitata (white and red varieties) sprouts. Journal of the Science of Food and Agriculture, 97(8), 2291-2299. https://doi.org/10.1002/jsfa.8037

Havko, N. E., Major, I. T., Jewell, J. B., Attaran, E., Browse, J., \& Howe, G. A. (2016). Control of Carbon Assimilation and Partitioning by Jasmonate: An Accounting of GrowthDefense Tradeoffs. Plants, 5(1), 7. https://doi.org/10.3390/plants5010007

He, L., Diedrich, J., Chu, Y.-Y., \& Yates, J. R. (2015). Extracting Accurate Precursor Information for Tandem Mass Spectra by RawConverter. Analytical Chemistry, 87(22), 11361-11367. https://doi.org/10.1021/acs.analchem.5b02721 
Hentrich, M., Böttcher, C., Düchting, P., Cheng, Y., Zhao, Y., Berkowitz, O., ... Pollmann, S. (2013). The jasmonic acid signaling pathway is linked to auxin homeostasis through the modulation of YUCCA8 and YUCCA9 gene expression. The Plant Journal : For Cell and Molecular Biology, 74(4), 626-637. https://doi.org/10.1111/tpj.12152

Hopkins, R. J., Dam, N. M. van, \& Loon, J. J. A. van. (2009). Role of Glucosinolates in InsectPlant Relationships and Multitrophic Interactions. Annual Review of Entomology, 54(1), 57-83. https://doi.org/10.1146/annurev.ento.54.110807.090623

Hoppe, R. A. (2014). Structure and Finances of U.S.Farms: Family Farm Report, 2014 Edition. 67.

HOSTS - A Database of the World's Lepidopteran Hostplants. (n.d.). Retrieved November 19, 2017, from http://www.nhm.ac.uk/our-science/data/hostplants/

Houston, K., Tucker, M. R., Chowdhury, J., Shirley, N., \& Little, A. (2016). The Plant Cell Wall: A Complex and Dynamic Structure As Revealed by the Responses of Genes under Stress Conditions. Frontiers in Plant Science, 7. https://doi.org/10.3389/fpls.2016.00984

Howe, G. A., \& Jander, G. (2008). Plant Immunity to Insect Herbivores. Annual Review of Plant Biology, 59(1), 41-66. https://doi.org/10.1146/annurev.arplant.59.032607.092825

Hu, R., Xu, C., Shen, G., Jain, M. R., Khor, T. O., Gopalkrishnan, A., .. Kong, A.-N. T. (2006). Gene expression profiles induced by cancer chemopreventive isothiocyanate sulforaphane in the liver of C57BL/6J mice and C57BL/6J/Nrf2 (-/-) mice. Cancer Letters, 243(2), 170-192. https://doi.org/10.1016/j.canlet.2005.11.050

Huot, B., Yao, J., Montgomery, B. L., \& He, S. Y. (2014). Growth-Defense Tradeoffs in Plants: A Balancing Act to Optimize Fitness. Molecular Plant, 7(8), 1267-1287. https://doi.org/10.1093/mp/ssu049 
Hwang, E.-S., \& Kim, G.-H. (2013). Effects of various heating methods on glucosinolate, carotenoid and tocopherol concentrations in broccoli. International Journal of Food Sciences and Nutrition, 64(1), 103-111. https://doi.org/10.3109/09637486.2012.704904

ISO. (1992). The international Organization of stanardization ISO 9167-1.

Jeschke, V., Gershenzon, J., \& Vassão, D. G. (2016). Chapter Eight - Insect Detoxification of Glucosinolates and Their Hydrolysis Products. In S. Kopriva (Ed.), Advances in Botanical Research (pp. 199-245). https://doi.org/10.1016/bs.abr.2016.06.003

Jiang, Y., Ye, J., Li, S., \& Niinemets, Ü. (2017). Methyl jasmonate-induced emission of biogenic volatiles is biphasic in cucumber: a high-resolution analysis of dose dependence. Journal of Experimental Botany, 68(16), 4679-4694. https://doi.org/10.1093/jxb/erx244

Jones, R. B., Frisina, C. L., Winkler, S., Imsic, M., \& Tomkins, R. B. (2010). Cooking method significantly effects glucosinolate content and sulforaphane production in broccoli florets. Food Chemistry, 123(2), 237-242. https://doi.org/10.1016/j.foodchem.2010.04.016

Jump, S. M., Kung, J., Staub, R., Kinseth, M. A., Cram, E. J., Yudina, L. N., ... Firestone, G. L. (2008). N-Alkoxy derivatization of indole-3-carbinol increases the efficacy of the G1 cell cycle arrest and of $\mathrm{I3C}$-specific regulation of cell cycle gene transcription and activity in human breast cancer cells. Biochemical Pharmacology, 75(3), 713-724. https://doi.org/10.1016/j.bcp.2007.09.024

Kapusta-Duch, J., Kusznierewicz, B., Leszczyńska, T., \& Borczak, B. (2016). Effect of cooking on the contents of glucosinolates and their degradation products in selected Brassica vegetables. Journal of Functional Foods, 23, 412-422. https://doi.org/10.1016/j.jff.2016.03.006 
Kim, H. S. (2010). Functional studies of lignin biosynthesis genes and putative flowering gene in Miscanthus x giganteus and studies on indolyl glucosinolate biosynthesis and translocation in Brassica oleracea (University of Illinois at Urbana-Champaign). Retrieved from http://search.proquest.com/openview/209f070009674bf11c82a9c2af39128f/1?pqorigsite $=$ gscholar $\& \mathrm{cbl}=18750 \&$ diss $=\mathrm{y}$

Kim, H. S., \& Juvik, J. A. (2011). Effect of Selenium Fertilization and Methyl Jasmonate Treatment on Glucosinolate Accumulation in Broccoli Florets. Journal of the American Society for Horticultural Science, 136(4), 239-246.

Kim, J. H., \& Jander, G. (2007). Myzus persicae (green peach aphid) feeding on Arabidopsis induces the formation of a deterrent indole glucosinolate. The Plant Journal, 49(6), 10081019. https://doi.org/10.1111/j.1365-313X.2006.03019.x

Kim, M. J., Chiu, Y.-C., Kim, N. K., Park, H. M., Lee, C. H., Juvik, J. A., \& Ku, K.-M. (2017). Cultivar-Specific Changes in Primary and Secondary Metabolites in Pak Choi (Brassica Rapa, Chinensis Group) by Methyl Jasmonate. International Journal of Molecular Sciences, 18(5), 1004. https://doi.org/10.3390/ijms18051004

Kim, M. J., Chiu, Y.-C., \& Ku, K.-M. (2017). Glucosinolates, Carotenoids, and Vitamins E and K Variation from Selected Kale and Collard Cultivars. Journal of Food Quality, 2017, 18. https://doi.org/10.1155/2017/5123572

Kliebenstein, D. J., Kroymann, J., Brown, P., Figuth, A., Pedersen, D., Gershenzon, J., \& Mitchell-Olds, T. (2001). Genetic Control of Natural Variation in Arabidopsis Glucosinolate Accumulation. Plant Physiology, 126(2), 811-825. https://doi.org/10.1104/pp.126.2.811 
Kochan, E., Balcerczak, E., Lipert, A., Szymańska, G., \& Szymczyk, P. (2018). Methyl jasmonate as a control factor of the synthase squalene gene promoter and ginsenoside production in American ginseng hairy root cultured in shake flasks and a nutrient sprinkle bioreactor. Industrial Crops and Products, 115, 182-193. https://doi.org/10.1016/j.indcrop.2018.02.036

Kohlmann, M., Bachmann, A., Weichert, H., Kolbe, A., Balkenhohl, T., Wasternack, C., \& Feussner, I. (1999). Formation of lipoxygenase-pathway-derived aldehydes in barley leaves upon methyl jasmonate treatment. European Journal of Biochemistry, 260(3), 885-895. https://doi.org/10.1046/j.1432-1327.1999.00231.x

Krueger, R. A. (2014). Focus Groups: A Practical Guide for Applied Research. SAGE Publications.

Ku, K. M., Becker, T. M., \& Juvik, J. A. (2016). Transcriptome and Metabolome Analyses of Glucosinolates in Two Broccoli Cultivars Following Jasmonate Treatment for the Induction of Glucosinolate Defense to Trichoplusia ni (Hübner). International Journal of Molecular Sciences, 17(7), 1135. https://doi.org/10.3390/ijms17071135

Ku, K. M., Choi, J. H., Kim, H. S., Kushad, M. M., Jeffery, E. H., \& Juvik, J. A. (2013). Methyl Jasmonate and 1-Methylcyclopropene Treatment Effects on Quinone Reductase Inducing Activity and Post-Harvest Quality of Broccoli. PLoS ONE, 8(10), e77127. https://doi.org/10.1371/journal.pone.0077127

Ku, K. M., Choi, J.-H., Kushad, M. M., Jeffery, E. H., \& Juvik, J. A. (2013). Pre-harvest Methyl Jasmonate Treatment Enhances Cauliflower Chemoprotective Attributes Without a Loss in Postharvest Quality. Plant Foods for Human Nutrition, 68(2), 113-117. https://doi.org/10.1007/s11130-013-0356-y 
Ku, K. M., Jeffery, E. H., \& Juvik, J. A. (2013). Influence of Seasonal Variation and Methyl Jasmonate Mediated Induction of Glucosinolate Biosynthesis on Quinone Reductase Activity in Broccoli Florets. Journal of Agricultural and Food Chemistry, 61(40), 96239631. https://doi.org/10.1021/jf4027734

Ku, K. M., Jeffery, E. H., \& Juvik, J. A. (2014a). Exogenous Methyl Jasmonate Treatment Increases Glucosinolate Biosynthesis and Quinone Reductase Activity in Kale Leaf Tissue. PLoS ONE, 9(8), e103407. https://doi.org/10.1371/journal.pone.0103407

Ku, K. M., Jeffery, E. H., \& Juvik, J. A. (2014b). Optimization of methyl jasmonate application to broccoli florets to enhance health-promoting phytochemical content: meja enhancement of health-promoting phytochemicals in broccoli. Journal of the Science of Food and Agriculture, 94(10), 2090-2096. https://doi.org/10.1002/jsfa.6529

Ku, K. M., Jeffery, E. H., Juvik, J. A., \& Kushad, M. M. (2015). Correlation of Quinone Reductase Activity and Allyl Isothiocyanate Formation Among Different Genotypes and Grades of Horseradish Roots. Journal of Agricultural and Food Chemistry, 63(11), 29472955. https://doi.org/10.1021/jf505591z

Ku, K. M., \& Juvik, J. A. (2013). Environmental Stress and Methyl Jasmonate-mediated Changes in Flavonoid Concentrations and Antioxidant Activity in Broccoli Florets and Kale Leaf Tissues. HortScience, 48(8), 996-1002.

Ku, K. M., Kim, M. J., Jeffery, E. H., Kang, Y.-H., \& Juvik, J. A. (2016). Profiles of Glucosinolates, Their Hydrolysis Products, and Quinone Reductase Inducing Activity from 39 Arugula (Eruca sativa Mill.) Accessions. Journal of Agricultural and Food Chemistry, 64(34), 6524-6532. https://doi.org/10.1021/acs.jafc.6b02750 
Kupke, F., Herz, C., Hanschen, F. S., Platz, S., Odongo, G. A., Helmig, S., ... Lamy, E. (2016). Cytotoxic and genotoxic potential of food-borne nitriles in a liver in vitro model. Scientific Reports, 6. https://doi.org/10.1038/srep37631

Kusznierewicz, B., Iori, R., Piekarska, A., Namieśnik, J., \& Bartoszek, A. (2013a). Convenient identification of desulfoglucosinolates on the basis of mass spectra obtained during liquid chromatography-diode array-electrospray ionisation mass spectrometry analysis: Method verification for sprouts of different Brassicaceae species extracts. Journal of Chromatography A, 1278, 108-115. https://doi.org/10.1016/j.chroma.2012.12.075

Kusznierewicz, B., Iori, R., Piekarska, A., Namieśnik, J., \& Bartoszek, A. (2013b). Convenient identification of desulfoglucosinolates on the basis of mass spectra obtained during liquid chromatography-diode array-electrospray ionisation mass spectrometry analysis: Method verification for sprouts of different Brassicaceae species extracts. Journal of Chromatography A, 1278, 108-115. https://doi.org/10.1016/j.chroma.2012.12.075 Lackman, P., González-Guzmán, M., Tilleman, S., Carqueijeiro, I., Pérez, A. C., Moses, T., ... Goossens, A. (2011). Jasmonate signaling involves the abscisic acid receptor PYL4 to regulate metabolic reprogramming in Arabidopsis and tobacco. Proceedings of the National Academy of Sciences, 108(14), 5891-5896. https://doi.org/10.1073/pnas.1103010108

Lambrix, V., Reichelt, M., Mitchell-Olds, T., Kliebenstein, D. J., \& Gershenzon, J. (2001). The Arabidopsis Epithiospecifier Protein Promotes the Hydrolysis of Glucosinolates to Nitriles and Influences Trichoplusia ni Herbivory. The Plant Cell Online, 13(12), 27932807. https://doi.org/10.1105/tpc.010261 
Lee-Kwan, S. H., Moore, L. V., Blanck, H. M., Harris, D. M., \& Galuska, D. (2017). Disparities in State-Specific Adult Fruit and Vegetable Consumption — United States, 2015. MMWR. Morbidity and Mortality Weekly Report, 66(45), 1241-1247. https://doi.org/10.15585/mmwr.mm6645a1

Li, N., Han, X., Feng, D., Yuan, D., \& Huang, L.-J. (2019). Signaling Crosstalk between Salicylic Acid and Ethylene/Jasmonate in Plant Defense: Do We Understand What They Are Whispering? International Journal of Molecular Sciences, 20(3), 671. https://doi.org/10.3390/ijms20030671

Lisec, J., Schauer, N., Kopka, J., Willmitzer, L., \& Fernie, A. R. (2006). Gas chromatography mass spectrometry-based metabolite profiling in plants. Nature Protocols, 1(1), 387-396. https://doi.org/10.1038/nprot.2006.59

Liu, H., Meng, F., Miao, H., Chen, S., Yin, T., Hu, S., ... Wang, Q. (2018). Effects of postharvest methyl jasmonate treatment on main health-promoting components and volatile organic compounds in cherry tomato fruits. Food Chemistry, 263, 194-200. https://doi.org/10.1016/j.foodchem.2018.04.124

Long, Y., Wang, J., Wang, Y., Zhang, J., Wang, J., \& Pei, X. (2016). Comparative analysis of MYB28 homologs and development of a MYB28-specific marker in Brassica napus L. Molecular Breeding, 36(9). https://doi.org/10.1007/s11032-016-0551-2

Luo, F., Cai, J.-H., Zhang, X., Tao, D.-B., Zhou, X., Zhou, Q., ... Ji, S.-J. (2018). Effects of methyl jasmonate and melatonin treatments on the sensory quality and bioactive compounds of harvested broccoli. RSC Advances, 8(72), 41422-41431. https://doi.org/10.1039/C8RA07982J 
Lv, J., Ge, Y., Li, C., Zhang, M., \& Li, J. (2017). Identification and Analysis of Genes Involved in the Jasmonate Pathway in Response to Ethephon and 1-Methylcyclopropene during the Ripening of Apple Fruit. Journal of the American Society for Horticultural Science, 142(3), 184-191. https://doi.org/10.21273/JASHS04054-17

Machado, R. A. R., Arce, C. C. M., Ferrieri, A. P., Baldwin, I. T., \& Erb, M. (2015). Jasmonatedependent depletion of soluble sugars compromises plant resistance to Manduca sexta. New Phytologist, 207(1), 91-105. https://doi.org/10.1111/nph.13337

Major, I. T., Yoshida, Y., Campos, M. L., Kapali, G., Xin, X.-F., Sugimoto, K., ... Howe, G. A. (2017). Regulation of growth-defense balance by the JASMONATE ZIM-DOMAIN (JAZ)-MYC transcriptional module. New Phytologist, 215(4), 1533-1547. https://doi.org/10.1111/nph.14638

Mao, Y.-B., Liu, Y.-Q., Chen, D.-Y., Chen, F.-Y., Fang, X., Hong, G.-J., ... Chen, X.-Y. (2017). Jasmonate response decay and defense metabolite accumulation contributes to ageregulated dynamics of plant insect resistance. Nature Communications, 8, ncomms13925. https://doi.org/10.1038/ncomms13925

Martin, N., \& Müller, C. (2007). Induction of plant responses by a sequestering insect: Relationship of glucosinolate concentration and myrosinase activity. Basic and Applied Ecology, 8(1), 13-25. https://doi.org/10.1016/j.baae.2006.02.001

Matusheski, N. V., \& Jeffery, E. H. (2001). Comparison of the Bioactivity of Two Glucoraphanin Hydrolysis Products Found in Broccoli, Sulforaphane and Sulforaphane Nitrile. Journal of Agricultural and Food Chemistry, 49(12), 5743-5749. https://doi.org/10.1021/jf010809a 
McEwen, C. L. (2011). Two entomological studies: 1. The potential of Methyl jasmonate applications as a pest management method on cruciferous crops 2 . Contributions to the biology of Disholcaspis quercusmamma (Walsh)(Hymenoptera: Cynipidae) (Master's Thesis). Colorado State University, Fort Collins, CO, USA.

Meilgaard, M. C., Carr, B. T., \& Civille, G. V. (2006). Sensory Evaluation Techniques, Fourth Edition. CRC Press.

Mennella, J. A., \& Bobowski, N. K. (2015). The sweetness and bitterness of childhood: Insights from basic research on taste preferences. Physiology \& Behavior, 152, 502-507. https://doi.org/10.1016/j.physbeh.2015.05.015

Mewis, I., Appel, H. M., Hom, A., Raina, R., \& Schultz, J. C. (2005). Major Signaling Pathways Modulate Arabidopsis Glucosinolate Accumulation and Response to Both PhloemFeeding and Chewing Insects. Plant Physiology, 138(2), 1149-1162. https://doi.org/10.1104/pp.104.053389

Mewis, I., Tokuhisa, J. G., Schultz, J. C., Appel, H. M., Ulrichs, C., \& Gershenzon, J. (2006). Gene expression and glucosinolate accumulation in Arabidopsis thaliana in response to generalist and specialist herbivores of different feeding guilds and the role of defense signaling pathways. Phytochemistry, 67(22), 2450-2462. https://doi.org/10.1016/j.phytochem.2006.09.004

Migliozzi, M., Thavarajah, D., Thavarajah, P., \& Smith, P. (2015). Lentil and Kale: Complementary Nutrient-Rich Whole Food Sources to Combat Micronutrient and Calorie Malnutrition. Nutrients, 7(11), 9285-9298. https://doi.org/10.3390/nu7115471

Mikkelsen, M. D., Hansen, C. H., Wittstock, U., \& Halkier, B. A. (2000). Cytochrome P450 CYP79B2 from Arabidopsis Catalyzes the Conversion of Tryptophan to Indole-3- 
acetaldoxime, a Precursor of Indole Glucosinolates and Indole-3-acetic Acid. Journal of Biological Chemistry, 275(43), 33712-33717. https://doi.org/10.1074/jbc.M001667200

Mohd Zul, S., \& Surugau, N. (2016). Effects of ascorbic acid and ferum ions concentration on the hydrolysis of flucosinolaye and myrosinase activity in the watercress (Nasturtium officinale sp.). Jurnal Teknologi, 78(8). https://doi.org/10.11113/jt.v78.5092

Moskowitz, H. R. (1970). Ratio scales of sugar sweetness. Perception \& Psychophysics, 7(5), 315-320. https://doi.org/10.3758/BF03210175

Müller, R., Vos, M. de, Sun, J. Y., Sønderby, I. E., Halkier, B. A., Wittstock, U., \& Jander, G. (2010). Differential Effects of Indole and Aliphatic Glucosinolates on Lepidopteran Herbivores. Journal of Chemical Ecology, 36(8), 905-913. https://doi.org/10.1007/s10886-010-9825-z

Mwaijande, F., Miller, J., Wailes, E., \& Peterson Jr., L. (2009). The Value of Focus Group Discussions for Understanding Barriers to Agriculture-Tourism Linkages in Developing Regions. Journal of International Agricultural and Extension Education, 16(3). https://doi.org/10.5191/jiaee.2009.16306

Napoleão, T. A., Soares, G., Vital, C. E., Bastos, C., Castro, R., Loureiro, M. E., \& Giordano, A. (2017). Methyl jasmonate and salicylic acid are able to modify cell wall but only salicylic acid alters biomass digestibility in the model grass Brachypodium distachyon. Plant Science, 263, 46-54. https://doi.org/10.1016/j.plantsci.2017.06.014

Neave, A. S., Sarup, S. M., Seidelin, M., Duus, F., \& Vang, O. (2005). Characterization of the Nmethoxyindole-3-carbinol (NI3C)-Induced Cell Cycle Arrest in Human Colon Cancer Cell Lines. Toxicological Sciences, 83(1), 126-135. https://doi.org/10.1093/toxsci/kfi008 
Ni Mhurchu, C., Eyles, H., Schilling, C., Yang, Q., Kaye-Blake, W., Genç, M., \& Blakely, T. (2013). Food Prices and Consumer Demand: Differences across Income Levels and Ethnic Groups. PLoS ONE, 8(10). https://doi.org/10.1371/journal.pone.0075934

Nishimura, T., \& Kato, H. (1988). Taste of free amino acids and peptides. Food Reviews International, 4(2), 175-194. https://doi.org/10.1080/87559128809540828

Okrent, A., \& Alston, J. (2012). The Demand for Disaggregated Food-Away-from-Home and Food-at-Home Products in the United States. SSRN Electronic Journal. https://doi.org/10.2139/ssrn.2171315

Oliviero, T., Verkerk, R., \& Dekker, M. (2018). Isothiocyanates from Brassica VegetablesEffects of Processing, Cooking, Mastication, and Digestion. Molecular Nutrition \& Food Research, 62(18), 1701069. https://doi.org/10.1002/mnfr.201701069

Osborne, J., \& Costello, A. (2004). Sample size and subject to item ratio in principal components analy. Retrieved March 16, 2018, from http://pareonline.net/htm/v9n11.htm

Palermo, M., Pellegrini, N., \& Fogliano, V. (2014). The effect of cooking on the phytochemical content of vegetables. Journal of the Science of Food and Agriculture, 94(6), 1057-1070. https://doi.org/10.1002/jsfa.6478

Poelman, A. A. M., Delahunty, C. M., \& de Graaf, C. (2013). Cooking time but not cooking method affects children's acceptance of Brassica vegetables. Food Quality and Preference, 28(2), 441-448. https://doi.org/10.1016/j.foodqual.2012.12.003

Reyes-Díaz, M., Lobos, T., Cardemil, L., Nunes-Nesi, A., Retamales, J., Jaakola, L., ... RiberaFonseca, A. (2016). Methyl Jasmonate: An Alternative for Improving the Quality and Health Properties of Fresh Fruits. Molecules, 21(6), 567. https://doi.org/10.3390/molecules21060567 
Rivera-Vega, L. J., Galbraith, D. A., Grozinger, C. M., \& Felton, G. W. (2017). Host plant driven transcriptome plasticity in the salivary glands of the cabbage looper (Trichoplusia ni). PLOS ONE, 12(8), e0182636. https://doi.org/10.1371/journal.pone.0182636

Rohwer, C. L., \& Erwin, J. E. (2008). Horticultural applications of jasmonates: A review. Román, J., Castillo, A., Cottet, L., \& Mahn, A. (2018). Kinetic and structural study of broccoli myrosinase and its interaction with different glucosinolates. Food Chemistry, 254, 87-94. https://doi.org/10.1016/j.foodchem.2018.01.179

Salkind, N. J. (Ed.). (2010). Encyclopedia of research design. Thousand Oaks, Calif: SAGE Publications.

Sarkar, F. H., \& Li, Y. (2004). Indole-3-Carbinol and Prostate Cancer. The Journal of Nutrition, 134(12), 3493S-3498S. https://doi.org/10.1093/jn/134.12.3493S

Sarvan, I., Kramer, E., Bouwmeester, H., Dekker, M., \& Verkerk, R. (2017). Sulforaphane formation and bioaccessibility are more affected by steaming time than meal composition during in vitro digestion of broccoli. Food Chemistry, 214, 580-586. https://doi.org/10.1016/j.foodchem.2016.07.111

Sarvan, Irmela, Verkerk, R., van Boekel, M., \& Dekker, M. (2014). Comparison of the degradation and leaching kinetics of glucosinolates during processing of four Brassicaceae (broccoli, red cabbage, white cabbage, Brussels sprouts). Innovative Food Science \& Emerging Technologies, 25, 58-66. https://doi.org/10.1016/j.ifset.2014.01.007

Schätzer, M., Rust, P., \& Elmadfa, I. (2010). Fruit and vegetable intake in Austrian adults: intake frequency, serving sizes, reasons for and barriers to consumption, and potential for increasing consumption. Public Health Nutrition, 13(4), 480-487. https://doi.org/10.1017/S136898000999142X 
Schuman, M. C., \& Baldwin, I. T. (2016). The Layers of Plant Responses to Insect Herbivores. Annual Review of Entomology, 61(1), 373-394. https://doi.org/10.1146/annurev-ento010715-023851

Seldal, T., Hegland, S. J., Rydgren, K., Rodriguez-Saona, C., \& Töpper, J. P. (2017). How to induce defense responses in wild plant populations? Using bilberry (Vaccinium myrtillus) as example. Ecology and Evolution, 7(6), 1762-1769. https://doi.org/10.1002/ece3.2687

Shapiro, T. A., Fahey, J. W., Wade, K. L., Stephenson, K. K., \& Talalay, P. (2001). Chemoprotective Glucosinolates and Isothiocyanates of Broccoli Sprouts Metabolism and Excretion in Humans. Cancer Epidemiology Biomarkers \& Prevention, 10(5), 501508.

Sikalidis, A. K. (2015). Amino Acids and Immune Response: A Role for Cysteine, Glutamine, Phenylalanine, Tryptophan and Arginine in T-cell Function and Cancer? Pathology \& Oncology Research, 21(1), 9-17. https://doi.org/10.1007/s12253-014-9860-0

Smith, C. A., Want, E. J., O’Maille, G., Abagyan, R., \& Siuzdak, G. (2006). XCMS: processing mass spectrometry data for metabolite profiling using nonlinear peak alignment, matching, and identification. Analytical Chemistry, 78(3), 779-787. https://doi.org/10.1021/ac051437y

Sønderby, I. E., Geu-Flores, F., \& Halkier, B. A. (2010). Biosynthesis of glucosinolates - gene discovery and beyond. Trends in Plant Science, 15(5), 283-290. https://doi.org/10.1016/j.tplants.2010.02.005

Song, L., \& Thornalley, P. J. (2007). Effect of storage, processing and cooking on glucosinolate content of Brassica vegetables. Food and Chemical Toxicology, 45(2), 216-224. https://doi.org/10.1016/j.fct.2006.07.021 
Song, S., Qi, T., Wasternack, C., \& Xie, D. (2014). Jasmonate signaling and crosstalk with gibberellin and ethylene. Current Opinion in Plant Biology, 21, 112-119. https://doi.org/10.1016/j.pbi.2014.07.005

Stephensen, P. U., Bonnesen, C., Schaldach, C., Andersen, O., Bjeldanes, L. F., \& Vang, O. (2000). N-Methoxyindole-3-Carbinol Is a More Efficient Inducer of Cytochrome P-450 1A1 in Cultured Cells Than Indol-3-Carbinol. Nutrition and Cancer, 36(1), 112-121. https://doi.org/10.1207/S15327914NC3601_15

Szczegielniak, J., Borkiewicz, L., Szurmak, B., Lewandowska-Gnatowska, E., Statkiewicz, M., Klimecka, M., ... Muszyńska, G. (2012). Maize calcium-dependent protein kinase (ZmCPK11): local and systemic response to wounding, regulation by touch and components of jasmonate signaling. Physiologia Plantarum, 146(1), 1-14. https://doi.org/10.1111/j.1399-3054.2012.01587.x

Tak, J.-H., \& Isman, M. B. (2017). Penetration-enhancement underlies synergy of plant essential oil terpenoids as insecticides in the cabbage looper, Trichoplusia ni. Scientific Reports, 7. https://doi.org/10.1038/srep42432

Tamogami, S., Noge, K., Abe, M., Agrawal, G. K., \& Rakwal, R. (2012). Methyl jasmonate is transported to distal leaves via vascular process metabolizing itself into JA-Ile and triggering VOCs emission as defensive metabolites. Plant Signaling \& Behavior, 7(11), 1378-1381. https://doi.org/10.4161/psb.21762

Textor, S., \& Gershenzon, J. (2009). Herbivore induction of the glucosinolate-myrosinase defense system: major trends, biochemical bases and ecological significance. Phytochemistry Reviews, 8(1), 149-170. https://doi.org/10.1007/s11101-008-9117-1 
Thaler, J. S., Stout, M. J., Karban, R., \& Duffey, S. S. (1996). Exogenous jasmonates simulate insect wounding in tomato plants (Lycopersicon esculentum) in the laboratory and field. Journal of Chemical Ecology, 22(10), 1767-1781. https://doi.org/10.1007/BF02028503

Thaler, Jennifer S., Humphrey, P. T., \& Whiteman, N. K. (2012). Evolution of jasmonate and salicylate signal crosstalk. Trends in Plant Science, 17(5), 260-270. https://doi.org/10.1016/j.tplants.2012.02.010

Tian, M., Hui, M., Thannhauser, T. W., Pan, S., \& Li, L. (2017). Selenium-Induced Toxicity Is Counteracted by Sulfur in Broccoli (Brassica oleracea L. var. italica). Frontiers in Plant Science, 8. https://doi.org/10.3389/fpls.2017.01425

Tian, S., Liu, X., Lei, P., Zhang, X., \& Shan, Y. (2018). Microbiota: a mediator to transform glucosinolate precursors in cruciferous vegetables to the active isothiocyanates. Journal of the Science of Food and Agriculture, 98(4), 1255-1260. https://doi.org/10.1002/jsfa.8654

Traka, M. H., Saha, S., Huseby, S., Kopriva, S., Walley, P. G., Barker, G. C., ... Mithen, R. F. (2013). Genetic regulation of glucoraphanin accumulation in Beneforté ${ }^{\circledR}$ broccoli. New Phytologist, 198(4), 1085-1095. https://doi.org/10.1111/nph.12232

Tsao, R., Peterson, C. J., \& Coats, J. R. (2002). Glucosinolate breakdown products as insect fumigants and their effect on carbon dioxide emission of insects. BMC Ecology, 2, 5. https://doi.org/10.1186/1472-6785-2-5

Turrell, G., Hewitt, B., Patterson, C., Oldenburg, B., \& Gould, T. (2002). Socioeconomic differences in food purchasing behaviour and suggested implications for diet-related health promotion. Journal of Human Nutrition and Dietetics, 15(5), 355-364. https://doi.org/10.1046/j.1365-277X.2002.00384.x 
USDC. (2019). State Personal Income, First Quarter 2019. State Personal Income, 11.

Vallejo, F., Tomás-Barberán, F., \& García-Viguera, C. (2002). Glucosinolates and vitamin C content in edible parts of broccoli florets after domestic cooking. European Food Research and Technology, 215(4), 310-316. https://doi.org/10.1007/s00217-002-0560-8

Velasco, P., Cartea, M. E., González, C., Vilar, M., \& Ordás, A. (2007). Factors Affecting the Glucosinolate Content of Kale (Brassica oleracea acephala Group). Journal of Agricultural and Food Chemistry, 55(3), 955-962. https://doi.org/10.1021/jf0624897

Wasternack, C., \& Hause, B. (2013). Jasmonates: biosynthesis, perception, signal transduction and action in plant stress response, growth and development. An update to the 2007 review in Annals of Botany. Annals of Botany, 111(6), 1021-1058. https://doi.org/10.1093/aob/mct067

Watanabe, K., Kamo, T., Nishikawa, F., \& Hyodo, H. (2000). Effect of Methyl Jasmonate on Senescence of Broccoli Florets. Journal of the Japanese Society for Horticultural Science, 69(5), 605-610. https://doi.org/10.2503/jjshs.69.605

Wathelet, J.-P., Marlier, M., Severin, M., Boenke, A., \& Wagstaffe, P. J. (1995). Measurement of glucosinolates in rapeseeds. Natural Toxins, 3(4), 299-304. https://doi.org/10.1002/nt.2620030425

Wieczorek, M., \& Jeleń, H. (2019). Volatile Compounds of Selected Raw and Cooked Brassica Vegetables. Molecules, 24(3), 391. https://doi.org/10.3390/molecules24030391

Wieczorek, M. N., Walczak, M., Skrzypczak-Zielińska, M., \& Jeleń, H. H. (2017). Bitter taste of Brassica vegetables: The role of genetic factors, receptors, isothiocyanates, glucosinolates, and flavor context. Critical Reviews in Food Science and Nutrition, 1-11. https://doi.org/10.1080/10408398.2017.1353478 
Wiesner, M., Hanschen, F. S., Schreiner, M., Glatt, H., \& Zrenner, R. (2013). Induced Production of 1-Methoxy-indol-3-ylmethyl Glucosinolate by Jasmonic Acid and Methyl Jasmonate in Sprouts and Leaves of Pak Choi (Brassica rapa ssp. chinensis). International Journal of Molecular Sciences, 14(7), 14996-15016. https://doi.org/10.3390/ijms 140714996

Williams, D. J., Critchley, C., Pun, S., Nottingham, S., \& O’Hare, T. J. (2008). Epithiospecifier protein activity in broccoli: The link between terminal alkenyl glucosinolates and sulphoraphane nitrile. Phytochemistry, 69(16), 2765-2773. https://doi.org/10.1016/j.phytochem.2008.09.018

Wittstock, U., Kliebenstein, D. J., Lambrix, V., Reichelt, M., \& Gershenzon, J. (2003). Chapter five Glucosinolate hydrolysis and its impact on generalist and specialist insect herbivores. Recent Advances in Phytochemistry, 37, 101-125. https://doi.org/10.1016/S00799920(03)80020-5

Wolucka, B. A., Goossens, A., \& Inzé, D. (2005). Methyl jasmonate stimulates the de novo biosynthesis of vitamin C in plant cell suspensions. Journal of Experimental Botany, 56(419), 2527-2538. https://doi.org/10.1093/jxb/eri246

Wu, G. (2010). Functional Amino Acids in Growth, Reproduction, and Health. Advances in Nutrition, 1(1), 31-37. https://doi.org/10.3945/an.110.1008

Wu, J., Wang, L., \& Baldwin, I. T. (2008). Methyl jasmonate-elicited herbivore resistance: does MeJA function as a signal without being hydrolyzed to JA? Planta, 227(5), 1161-1168. https://doi.org/10.1007/s00425-008-0690-8 
Wu, W., Yuan, M., Zhang, Q., Zhu, Y., Yong, L., Wang, W., ... Guo, D. (2011). Chemotypedependent Metabolic Response to Methyl Jasmonate Elicitation in Artemisia annua. Planta Medica, 77(10), 1048-1053. https://doi.org/10.1055/s-0030-1250744

WV News. (2019). West Virginia's economy slowly improving, though growth is concentrated in just seven counties. Retrieved September 12, 2019, from WV News website: https://www.wvnews.com/news/wvnews/west-virginia-s-economy-slowly-improvingthough-growth-is-concentrated/article_6b0d609a-d7c2-56a8-9128-b08db31386bf.html

Xia, J., \& Wishart, D. S. (2002). Using MetaboAnalyst 3.0 for Comprehensive Metabolomics Data Analysis. In Current Protocols in Bioinformatics. https://doi.org/10.1002/cpbi.11

Yi, G.-E., Robin, A. H. K., Yang, K., Park, J.-I., Hwang, B. H., \& Nou, I.-S. (2016). Exogenous Methyl Jasmonate and Salicylic Acid Induce Subspecies-Specific Patterns of Glucosinolate Accumulation and Gene Expression in Brassica oleracea L. Molecules, 21(10), 1417. https://doi.org/10.3390/molecules21101417

Yi, G.-E., Robin, A. H. K., Yang, K., Park, J.-I., Kang, J.-G., Yang, T.-J., \& Nou, I.-S. (2015). Identification and Expression Analysis of Glucosinolate Biosynthetic Genes and Estimation of Glucosinolate Contents in Edible Organs of Brassica oleracea Subspecies. Molecules, 20(7), 13089-13111. https://doi.org/10.3390/molecules200713089

Zabaras, D., Cox, D. N., Konczak, I., Roohani, M., Melo, L., Krishnamurthy, R., ... Delahunty, C. (2018). Taste and health promoting factors of Brassica bioactive compounds. 112. Zang, Y., Zhang, H., Huang, L., Wang, F., Gao, F., Lv, X., ... Zhu, Z. (2016). Glucosinolate enhancement in leaves and roots of pak choi (Brassica rapa ssp. chinensis) by methyl jasmonate. Horticulture, Environment, and Biotechnology, 56(6), 830-840. https://doi.org/10.1007/s13580-015-0079-0 
Zang, Y., Zheng, W., He, Y., Hong, S.-B., \& Zhu, Z. (2015). Global analysis of transcriptional response of Chinese cabbage to methyl jasmonate reveals JA signaling on enhancement of secondary metabolism pathways. Scientia Horticulturae, 189, 159-167. https://doi.org/10.1016/j.scienta.2015.04.008

Zhang, X., Shu, X.-O., Xiang, Y.-B., Yang, G., Li, H., Gao, J., ... Zheng, W. (2011). Cruciferous vegetable consumption is associated with a reduced risk of total and cardiovascular disease mortality. The American Journal of Clinical Nutrition, ajcn.009340. https://doi.org/10.3945/ajen.110.009340

Zhang, Y, Talalay, P., Cho, C. G., \& Posner, G. H. (1992). A major inducer of anticarcinogenic protective enzymes from broccoli: isolation and elucidation of structure. Proceedings of the National Academy of Sciences of the United States of America, 89(6), 2399-2403.

Zhang, Yuanyuan, Huai, D., Yang, Q., Cheng, Y., Ma, M., Kliebenstein, D. J., \& Zhou, Y. (2015). Overexpression of Three Glucosinolate Biosynthesis Genes in Brassica napus Identifies Enhanced Resistance to Sclerotinia sclerotiorum and Botrytis cinerea. PLOS ONE, 10(10), e0140491. https://doi.org/10.1371/journal.pone.0140491

Zhang, Yuesheng. (2010). Allyl isothiocyanate as a cancer chemopreventive phytochemical. Molecular Nutrition \& Food Research, 54(1), 127. https://doi.org/10.1002/mnfr.200900323

Zhang, Z., Ober, J. A., \& Kliebenstein, D. J. (2006). The Gene Controlling the Quantitative Trait Locus EPITHIOSPECIFIER MODIFIER1 Alters Glucosinolate Hydrolysis and Insect Resistance in Arabidopsis. The Plant Cell, 18(6), 1524-1536. https://doi.org/10.1105/tpc.105.039602 
Zhao, Y., Hull, A. K., Gupta, N. R., Goss, K. A., Alonso, J., Ecker, J. R., ... Celenza, J. L. (2002). Trp-dependent auxin biosynthesis in Arabidopsis: involvement of cytochrome P450s CYP79B2 and CYP79B3. Genes \& Development, 16(23), 3100-3112. https://doi.org/10.1101/gad.1035402

Zhou, S., Lou, Y.-R., Tzin, V., \& Jander, G. (2015). Alteration of Plant Primary Metabolism in Response to Insect Herbivory. Plant Physiology, 169(3), 1488-1498. https://doi.org/10.1104/pp.15.01405

Zhu, Z., \& Tian, S. (2012). Resistant responses of tomato fruit treated with exogenous methyl jasmonate to Botrytis cinerea infection. Scientia Horticulturae, 142, 38-43. https://doi.org/10.1016/j.scienta.2012.05.002 